University of Louisville

ThinkIR: The University of Louisville's Institutional Repository

$12-2012$

\title{
Intracellular delivery of nanomaterials for sub-cellular imaging and tracking of biomolecules.
}

Krishna Kiran Medepalli

University of Louisville

Follow this and additional works at: https://ir.library.louisville.edu/etd

\section{Recommended Citation}

Medepalli, Krishna Kiran, "Intracellular delivery of nanomaterials for sub-cellular imaging and tracking of biomolecules." (2012). Electronic Theses and Dissertations. Paper 958.

https://doi.org/10.18297/etd/958

This Doctoral Dissertation is brought to you for free and open access by ThinkIR: The University of Louisville's Institutional Repository. It has been accepted for inclusion in Electronic Theses and Dissertations by an authorized administrator of ThinkIR: The University of Louisville's Institutional Repository. This title appears here courtesy of the author, who has retained all other copyrights. For more information, please contact thinkir@louisville.edu. 


\title{
INTRACELLULAR DELIVERY OF NANOMATERIALS FOR SUB-CELLULAR IMAGING AND TRACKING OF BIOMOLECULES
}

\author{
By \\ Krishna Kiran Medepalli \\ M.S. Electrical Engineering, University of Louisville, \\ Louisville, Kentucky, 2008
}

\begin{abstract}
A Dissertation
Submitted to the Faculty of the

J.B. Speed School of Engineering, University of Louisville in Partial Fulfillment of the Requirements

for the Degree of

Doctor of Philosophy

Department of Electrical and Computer Engineering

University of Louisville

Louisville, Kentucky
\end{abstract}

December 2012 
Copyright 2012 by Krishna Kiran Medepalli

All rights reserved 
INTRACELLULAR DELIVERY OF NANOMATERIALS FOR SUB-CELLULAR IMAGING AND TRACKING OF BIOMOLECULES

\author{
By \\ Krishna Kiran Medepalli \\ M.S. Electrical Engineering, University of Louisville, \\ Louisville, Kentucky, 2008 \\ A Dissertation Approved on
}

November 26, 2012

by the following Dissertation Committee:

Dr. Bruce W. Alphenaar (Dissertation Director)

Dr. Palaniappan Sethu

Dr. Robert S. Keynton

Dr. Cindy K. Harnett

Dr. Shamus McNamara 


\section{DEDICATION}

This dissertation is dedicated to my parents

Mr. SRINIVASA RAO MEDEPALLI

$\&$

Mrs. GEETHANJALI MEDEPALLI

who are present in every step on my life.

And my wife

SILPA

who always gave me unconditional love and support without whom it would not be possible. 


\section{ACKNOWLEDGEMENTS}

This dissertation is the result of four long years of my doctoral research. Undoubtedly these are the most memorable years of my life. I am grateful to a lot of people for helping me professionally and personally to be successful in my graduate career.

I am greatly indebted to my advisor, Dr. Bruce Alphenaar for giving me the wonderful opportunity to work under his excellent guidance throughout my master and doctoral studies. I am extremely grateful to Dr. Palaniappan Sethu for his hands-on guidance and continued support throughout my doctoral research. I had the rare opportunity to be part of two of the best research laboratories at UofL and work in very interesting multidisciplinary projects. Their invaluable mentorship has not only taught me good research methods, scientific writing and presentation skills, but also inspired me to strive for more exciting results always. I can surely say that I will miss the time spent with them during my graduate career.

Another important person is Dr. Robert Keynton. I will always be grateful to him

for providing me the funding support during the latter half of my doctoral research and also for being part of my dissertation committee. I am also very thankful to Dr. Cindy Harnett and Dr. Shamus McNamara for their valuable time in reviewing my dissertation 
and being part of my committee. I would like to acknowledge Dr. Ashok Raj and Tim Andrews at the Division of Pediatrics (UofL) for providing us the samples. I would also like to thank University of Louisville for awarding me the prestigious university fellowship and also doctoral dissertation completion award during course of my doctoral studies.

Apart from my committee, I would like to thank Dr. Rosendo Estrada, Mai Dung and William White at the Microscale Biotechnology laboratory for familiarizing me with the flow cytometer and confocal microscope and for the interesting scientific discussions I had with them. I would also like to thank George, Buddika, Kasun and Sowmya who made my stay in the lab very enjoyable.

On personal front, this dissertation would not be possible without the constant love and support of my wife Silpa. Finally, I feel greatly indebted to my parents, in-laws, my sisters and sister-in-law, my cheerful nephew and four nieces and my best friends for everything they have done for me. 


\begin{abstract}
INTRACELLULAR DELIVERY OF NANOMATERIALS FOR SUB-CELLULAR IMAGING AND TRACKING OF BIOMOLECULES

Krishna Kiran Medepalli
\end{abstract}

November 26, 2012

Nanomaterials have many intriguing applications in biology and medicine. Unique properties such as enhanced electrical properties, increased chemical reactivity and resistance to degradation, novel optical properties and comparable size to that of biological systems have led to their use in various biomedical applications. The most important applications of nanomaterials for medicine are in drug delivery and imaging. This research focuses on utilizing the biocompatibility of single walled Carbon nanotubes (SWCNTs) and optical properties colloidal quantum dots (QDs) for cellular drug delivery and imaging of biomolecules.

The first part of this research deals with single walled carbon nanotubes which are excellent candidates for targeted drug delivery applications due their unique structural and functional properties. However, prior to their use in therapeutics, their biocompatibility needs to be thoroughly investigated. The objectives of this research were to establish the biocompatibility of SWCNTs and demonstrate their use as drug delivery 
carriers into cells. Blood, a living tissue, is chosen as the biological system as it contains various cells which can potentially interact with SWCNTs during the delivery mechanism. The interactions of these cells in the blood (specifically white blood cells or leukocytes) with the SWCNTs provide vital information regarding the immune response of the host to the nanotubes. This research investigates the immune response of white blood cells due to SWCNTs via (a) direct interaction - presence of nanotubes in the blood and, (b) indirect interaction - presentation of nanotubes by antigen-presenting-cells to white blood cells. These two interactions recreate the innate and adaptive immune responses occurring in the body to any foreign substance. SWCNTs are functionalized with single stranded DNA (ss-DNA), which serves as a dispersant of nanotubes as well as a backbone for further attachment of other biomolecules of interest. Confocal microscopy and flow cytometric studies are performed to characterize the interactions. Results from this acute immune response study demonstrate the biocompatibility of SWCNTs in whole blood and also confirm the cellular delivery of single stranded DNA.

The second part of the research is on colloidal quantum dots (QDs): nanometer sized semiconductor crystals typically between $1 \mathrm{~nm}$ to $20 \mathrm{~nm}$ in diameter. In addition to being size comparable with many biological systems, and having large surface area for multiple biomolecules attachment, they possess high resistance to chemical and photo degradation, tunable emission based on size and composition which makes them excellent candidates for cellular delivery and imaging. The main objectives of this research was to demonstrate the use of QDs for cellular imaging as well as targeted biomolecule delivery by conjugating the QDs with an antibody to a functional protein and delivery into live cells. Conventional techniques deliver QDs as aggregates, however, 
a major challenge in the use of QDs for cellular imaging and biomolecule delivery is acheiving freely dispersed QDs inside the cells. In this research, a new technique to deliver monodispersed QDs inside live cells was developed. The approach combines osmosis driven fluid transport into cells achieved by creating hypotonic environment and reversible permeabilization using low concentrations of cell permeabilization agents like Saponin. The results confirm that highly efficient endocytosis-free intracellular delivery of QDs can be accomplished using this method. Confocal microscopy is used to image the QDs inside the cells and flow cytometry is used for quantifying the fluorescence. To demonstrate targeted delivery, QDs are conjugated to the antibody of a protein: the nuclear transcriptional factor, NFkB (Nuclear Factor kappa-light chain-enhancer of activated B cells) using EDC/sulfo NHS chemistry methods. NFkB is a family of proteins with 5 different subunits and is involved in a variety of biological processes such as immune and inflammatory responses and cellular developmental processes. In unstimulated cells, NFkB is inactive in cytoplasm and translocates to the nucleus upon stimulation using bacterial products, viruses, radiation, and the like. QDs fluorescence could be used to monitor NFKB activity over extended periods of time in live cells. 


\section{TABLE OF CONTENTS}

PAGE

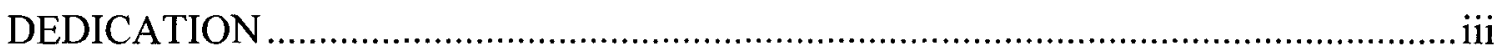

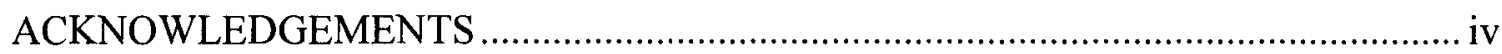

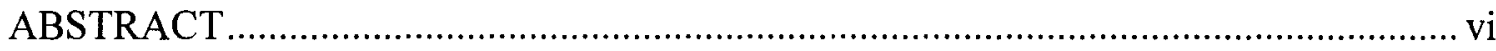

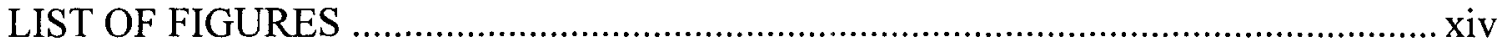

\section{CHAPTERS}

I. INTRODUCTION ................................................................................................................. 1

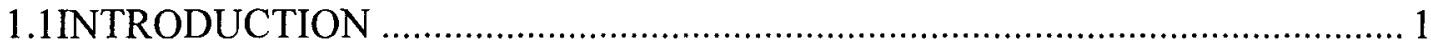

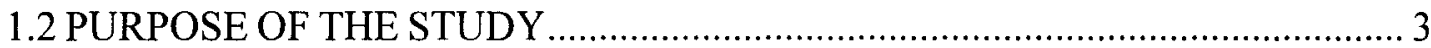

II. CARBON NANOTUBES.................................................................................................. 6

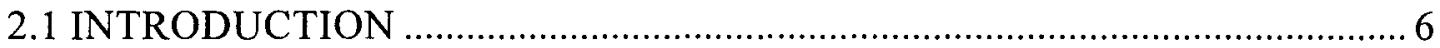

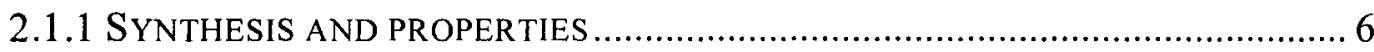

2.2 CNTS IN THE BIOMEDICAL FIELD ........................................................... 11

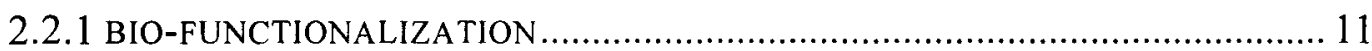

2.2.2 BIOLOGICAL APPLICATIONS .................................................................... 14

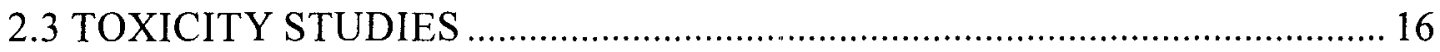

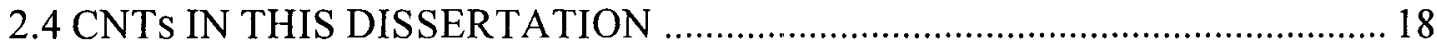

III. INTERACTION OF SWCNTS WITH BLOOD LEUCKOCYTES.................... 19 


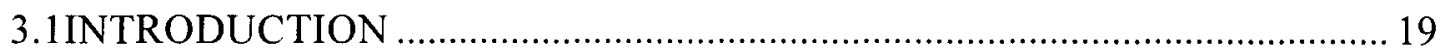

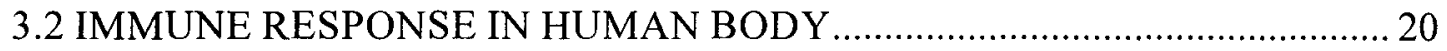

3.3 CELLS IN THE IMMUNE SYSTEM …................................................... 22

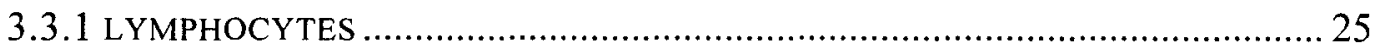

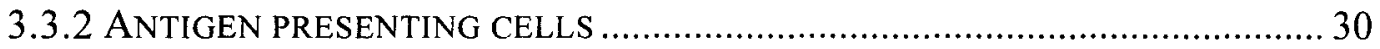

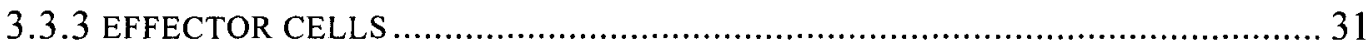

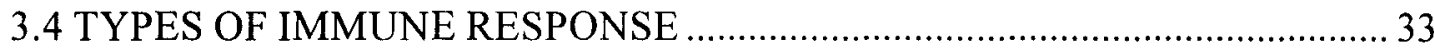

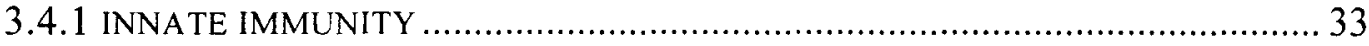

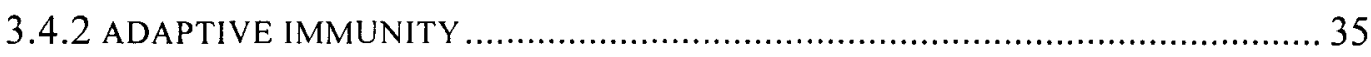

3.5 EVALUATION OF IMMUNE RESPONSE TO CNTS …............................. 37

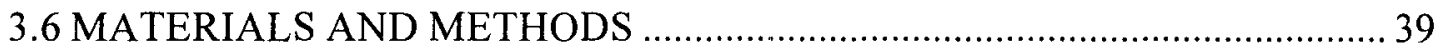

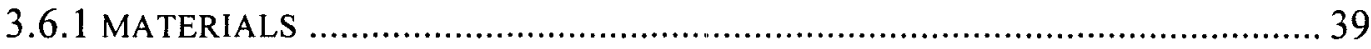

3.6.2 PREPARATION OF SWCNTS-DNA DISPERSIONS ................................ 40

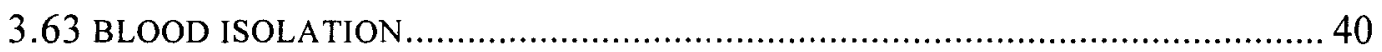

3.6.4 ISOLATION OF THE PBMCS FROM WHOLE BLOOD ................................. 40

3.6.5 ISOLATION OF LEUKOCYTES FROM WHOLE BLOOD ............................. 43

3.6.6 MONOCYTE/MACROPHAGE MATURATION STUDY ................................ 43

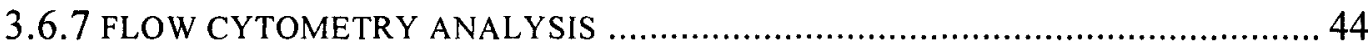

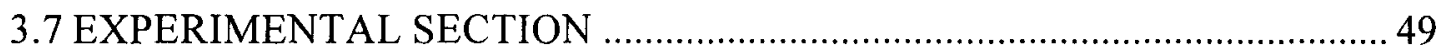

3.7.1 DIRECT INTERACTION OF SWCNTS WITH BLOOD ................................. 50

3.7.2 INDIRECT INTERACTION OF SWCNTS WITH BLOOD .............................. 52

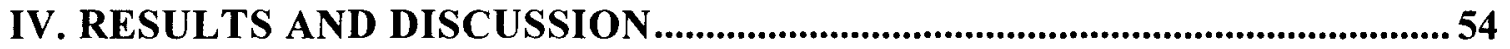

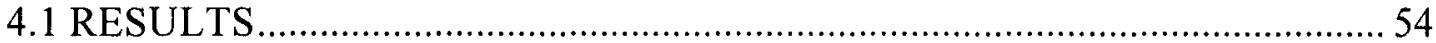


4.1.1 MONOCYTE ISOLATION AND MATURATION INTO APCS PHENOTYPE.......... 54

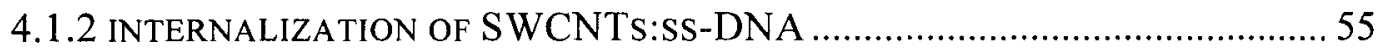

4.1.3 DIRECT INTERACTION OF SWCNTS WITH BLOOD .................................. 56

4.1.4 INDIRECT INTERACTION OF SWCNTS WITH BLOOD ...............................6 63

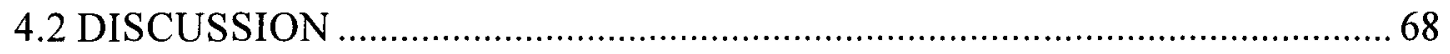

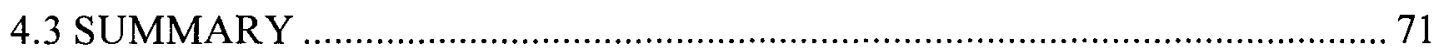

V. COLLOIDAL QUANTUM DOTS ..................................................................... 72

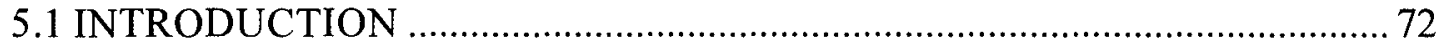

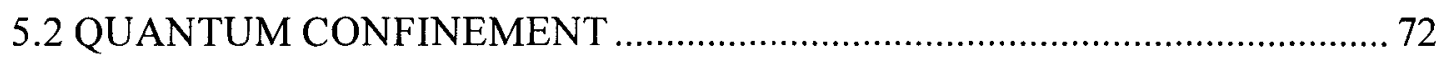

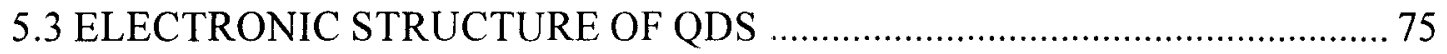

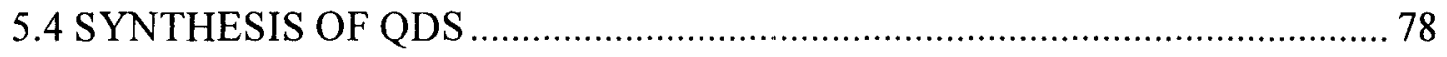

5.4.1 SURFACE MODIFICATION OF QDS FOR BIOCOMPATIBILITY ..................... 86

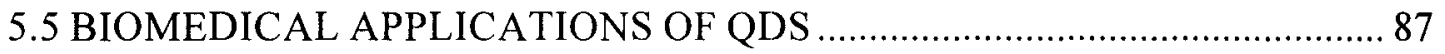

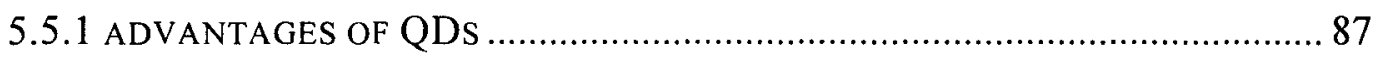

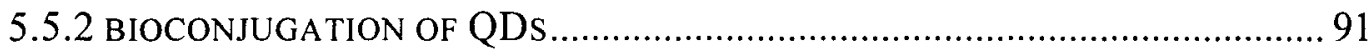

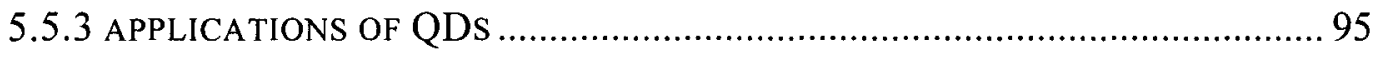

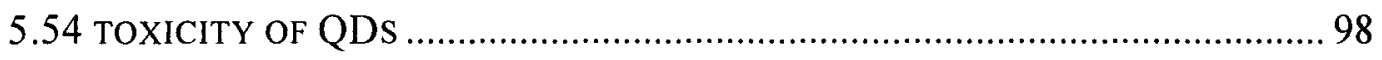

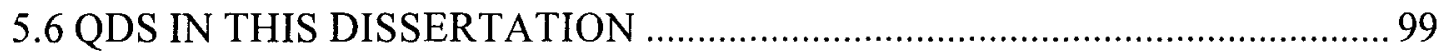

VI. REVERSIBLE PERMEABILIZATION FOR INTRACELLULAR

DELIVERY OF QDS ............................................................................................ 101

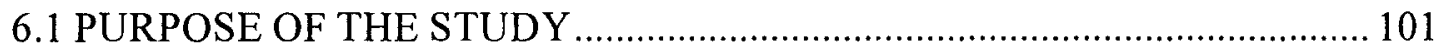

6.2 INTRACELLULAR DELIVERY OF QDS ........................................... 104

6.2.1 CURRENT INTRACELLULAR DELIVERY METHODS .................................. 104 
6.2.2 REVERSIBLE PERMEABILIZATION OF LIVE CELLS ................................... 110

6.3 EXPERIMENTAL MATERIALS AND METHODS ..................................... 113

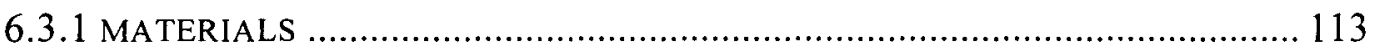

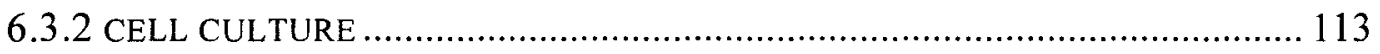

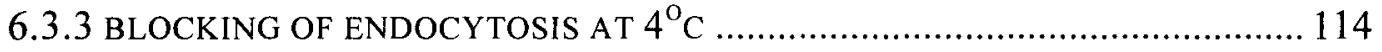

6.3.4 REVERSIBLE PERMEABILIZATION USING HYPOTONIC BUFFER ................... 114

6.3.5 REVERSIBLE PERMEABILIZATION USING SAPONIN .................................. 115

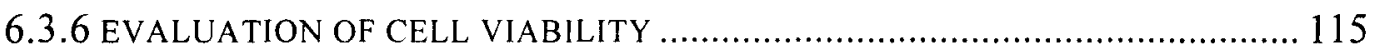

6.3.7 SIGNIFICANCE OF HYPOTONIC BUFFER CONDITIONS ................................. 116

6.3.8 QUANTITATIVE AND QUALITATIVE ANALYSIS USING FLUORESCENCE

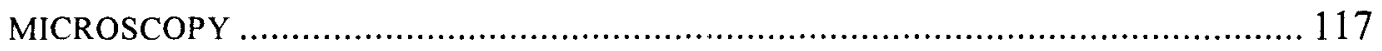

6.3.9 QUANTITATIVE ANALYSIS USING FLOW CYTOMETRY .............................. 117

6.4 QDS: NF-KB P65 ANTIBODY CONJUGATES ........................................... 117

6.4.1 STIMULATION OF CELLS FOR ACTIVATION OF NF-KB ............................ 118

6.4.2 IMMUNOFLUORESCENCE MICROSCOPY ……....................................... 118

VII. RESULTS AND DISCUSSION ........................................................................... 120

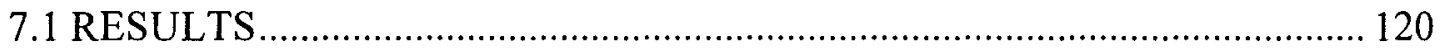

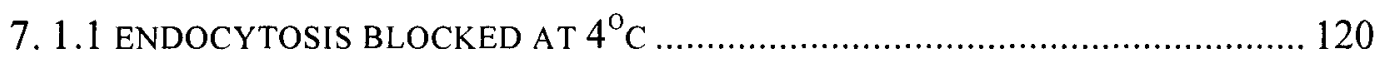

7.1.2 REVERSIBLE PERMEABILIZATION USING HYPOTONIC BUFFER .................. 123

7.1.3 REVERSIBLE PERMEABILIZATION USING SAPONIN .................................. 129

7.1.4 REVERSIBLE PERMEABILIZATION USING SAPONIN WITHOUT HYPOTONIC

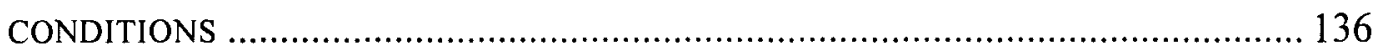

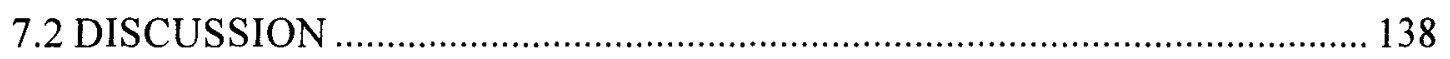


7.3 QDS: NF-KB P65 ANTIBODY CONJUGATES

7.3.1 STIMULATION OF CELLS FOR ACTIVATION AND NUCLEAR TRANSLOCATION

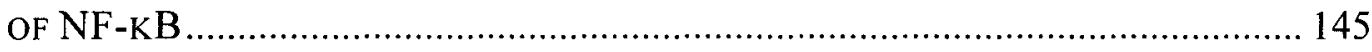

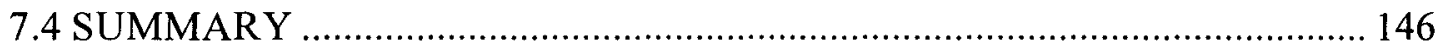

VIII. CONCLUSIONS AND FUTURE DIRECTIONS.......................................... 148

8.1 CONCLUSIONS

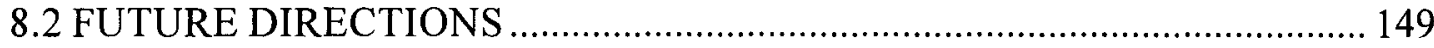

REFERENCES

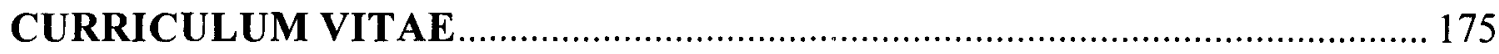




\section{LIST OF FIGURES}

FIGURE

PAGE

2.1 High resolution TEM images of MWCNTs formed by (a) five, (b) two, (c) seven graphene sheets.

2.2 Diagram showing the 2D graphene sheet and the vector structure classification to define CNTs structure using the chiral indices $(n, m)$. Open circles represent semiconducting and solid circles represent metallic CNTs respectively.

2.3 Density of states of (a) armchair CNT $(5,5)$ exhibiting metallic character and (b) zigzag CNT $(7,0)$ with semiconducting in nature.

2.4 Bio-functionalization of CNTs using (a) covalent functionalization, (b) noncovalent interactions, (c, d) hybrid approach

2.5 Simulation showing the wrapping of DNA on a SWCNT. The nuclei acid bases are color coded red while the sugar phosphate backbone is depicted in yellow. .14

3.1 Schematic representation of immunological memory function of immune system. 23

3.2 Anatomy of the major human lymphoid organs. The thymus and bone marrow (yellow) form the primary lymphoid organs. The other organs (blue) form the peripheral or the secondary lymphoid organs .24

3.3 Cells of the immune system derive from precursors in the bone marrow (pluripotent hematopoietic stem cell). .25

3.4 The fundamental cells of the immune system. The major cells that mediate immune responses along with their functions are shown. Microscopy images of some cells are shown in the left panel.

3.5 The different types of lymphocytes. Different classes of lymphocytes recognize antigens and differentiate into effector cells which help remove the antigens. B cells secrete antibodies to fight infection. $T$ helper cells recognize antigens on the surfaces of APCs and secrete cytokines. Cytotoxic T cells directly kill the infected 
cells. NK cells recognize infected cells via receptors and kill the infected cells.

3.6 Schematic showing the development and activation of T cells and B cells .30

3.7 Stages in the maturation of mononuclear phagocytes.Monocytes are derived from bone marrow stem cells differentiate into macrophages in tissues. Macrophages may be activated in the tissues in response to different antigens and may differentiate into specialized forms in different tissues. A light and an electron microscopy image of the macrophage is shown in the lower left panel. An electron microscopy image of an activated macrophage is shown in the lower right. .32

3.8 Schematic of the fundamental mechanisms involved in innate and adaptive immunity. The kinetics of the innate and adaptive immune response varies

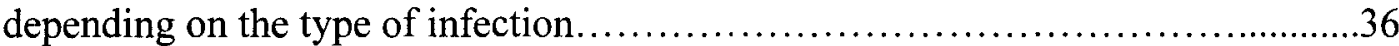

3.9 Innate immune response helps in the activation of adaptive immune response. .38

3.10 Isolation of PBMCs from whole blood. Schematic showing (a) layering of blood over ficoll before centrifugation, (b) the different layers after the density gradient centrifugation process, and (c) Representative photo of actual blood sample with isolated PBMCs. (Millipore).

3.11 Schematic of flow cytometer showing the major four components - fluidic system, laser(s), optical system and the electronics for data analysis. (BD biosciences).

3.12 Schematic of light scattering and refraction of a cell flowing through the flow cell. (a) forward scatter, and (b) side scatter from individual cell (BD biosciences).

3.13 Representative dot plot from a typical blood sample showing three distinct cell populations depicted as three regions. R1 (red) represent the lymphocytes, R2 (green) represents the monocytes and R3 (blue) represents granulocytes 48

3.14 Schematic of BD FACS calibur flow cytometer system showing the optical system layout. (BD biosciences). .49

3.15 Phenotype (blue) and activation (grey) markers used to label the different subpopulation of blood leukocytes. .51

4.1 Monocytes/macrophage maturation study. SWCNTs internalized monocytes are tracked for using maturation marker (CD 83). The percentage of CD83 ${ }^{\text {high }}$ cells is shown for a sample size of 4 . .55 
4.2 Confocal microscopy images of single monocytes with and without internalized SWCNTs:ss-DNA. Phase contrast and fluorescence images of cells (A), (B) without SWCNTs (control) and (C), (D) with SWCNTs functionalized with FAM-Q labeled ss-DNA. All the images are obtained at $60 \mathrm{x}$ magnification. .56

4.3 Direct interaction of SWCNTs with lymphocytes in blood. Representative sample comparing expressions of CD1a and CD69 on (A) CD3+CD4+ lymphocytes, (B) CD3+CD4- lymphocytes and (C) CD3-CD4- lymphocytes in whole blood (negative control) vs. SWCNT-stimulated cells. All histograms represent mean fluorescence intensity (arbitrary units) for the specific activation marker .59

4.4 Direct interaction experiment lymphocytes controls. Representative sample comparing expressions of CD1a and CD69 on (A) CD3+CD4+ lymphocytes, (B) CD3+CD4- lymphocytes and (C) CD3-CD4- lymphocytes in whole blood (negative control) vs. PMA (positive control)stimulated cells. All histograms represent mean fluorescence intensity (arbitrary units) for the specific activation marker. 60

4.5 Direct interaction of SWCNTs with monocytes in blood. Representative sample comparing expressions of CD11b, HLA-DR and CD18 markers on CD14+ monocytes in (A) whole blood (negative control) vs. SWCNTs-stimulated and (B) whole blood (negative control) vs. PMA-stimulated (positive control) blood samples. All histograms represent mean fluorescence intensity (arbitrary units) for the specific activation marker. .61

4.6 Direct interaction of SWCNTs with granulocytes in blood. Representative sample comparing expressions of $\mathrm{CD} 11 \mathrm{~b}, \mathrm{CD} 18$ and $\mathrm{CD} 69$ markers on CD66b+ granulocytes in (A) whole blood (negative control) vs. SWCNTs-stimulated and (B) whole blood (negative control) vs. PMA-stimulated (positive control) blood samples. All histograms represent mean fluorescence intensity (arbitrary units) for the specific activation marker.

4.7 Indirect interaction of SWCNTs with lymphocytes in blood. Representative sample comparing expressions of CD1a and CD69 on (A) CD3+CD4+, (B) CD3+CD4- and (C) CD3-CD4- lymphocytes in whole blood, stimulated with macrophage without internalized SWCNTS and with macrophages with SWCNTS. All histograms represent mean fluorescence intensity (arbitrary units) for the specific activation marker.

4.8 Indirect interaction of SWCNTs to monocytes and granulocytes in blood. Representative sample comparing expressions of (A) CD11b, HLA-DR and CD18 on CD14+ monocytes and (B) CD11b, CD69 and CD18 on CD66b+ granulocytes in whole blood, stimulated with macrophage without internalized SWCNTs and stimulated with macrophage with SWCNTs respectively. All histograms represent mean fluorescence intensity (arbitrary units) for the specific activation

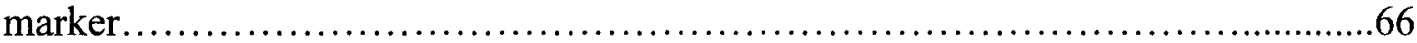


5.1 Density of states comparison of (A) metal and (B) semiconductor nanocrystals with respective bulk materials. The density of states is discrete in both metals and semiconductors near the band edges. In metal nanocrystals, as fermi level lies in the center of band, a few kT is greater than the energy level spacing and hence it resembles continuous states even for small sizes. However, in semiconductor nanocrystals, as the fermi level lies in between two bands, the energy level spacing is large even at large sizes and the energy gap increases in semiconductor nanocrystals of smaller size. . .73

5.2 Schematic of (a) 3D or bulk, (b) 2D or quantum well, (c) 1D or quantum wire, and (d) OD or quantum dot is shown. .76

5.3 Densities of states of (a) 3D or bulk, (b) 2D or quantum well, (c) 1D or quantum wire, and (d) OD or quantum dot. The DOS is continuous in bulk, a step function in QW, has peculiarities in QWR and a delta function in QDs .77

5.4 Comparison of discrete energy levels of two different QDs with (a) large radius and (b) small radius. As the size of the quantum dot decreases, the energy level spacing increases.

5.5 (A) La Mer model143 schematic of the different stages of nucleation and growth for the synthesis of of monodisperse nanocrystals. As the nanocrystals grow with time, different sized crystals are isolated by periodically removing aliquots from the reaction chamber, (B) Typical apparatus used in the synthesis of monodisperse QDs samples.

5.6 (A) Schematic of the energy band alignment of (A) type I, (B and C) type II and (D) Reverse type I core/shell QDs. CB and VB stands for conduction and valence bands respectively. Eg stands for bandgap of the semiconductor. The solid circle represents an electron and the empty circle a hole.

5.7 Schematic of the strategies employed for solubilization and biofunctionalization of QDs (a-h)..... 88

5.8 (A) Size tunable fluorescence emission of CdSe QDs, (B) relative sizes of QDs with diameters of $2.1 \mathrm{~nm}, 2.5 \mathrm{~nm}, 2.9 \mathrm{~nm}, 4.7 \mathrm{~nm}$ and $7.5 \mathrm{~nm}$.

5.9 QDs composition scaled as a function of the fluorescence emission wavelengths from ultraviolet to near infrared wavelengths. 90

5.10 Comparison of the absorption (blue) and fluorescence spectra (red) of CdSe QDs with fluorescence proteins (mCherry) and organic dye (Texas red). The absorption spectrum of QDs is broad and the emission spectrum is symmetric and narrow....91

5.11 Comparison of the photobleaching properties of QDs and organic dye (Texas Red) under similar excitation conditions. 
5.13 Typical methods of QDs bioconjugation. (a) Covalent crosslinking of antibody with QDs using EDAC chemistry method, (b) conjugation of antibody fragments using SMCC method, (c) conjugation of QDs using adaptor protein method and (d) conjugation of Ni-NTA modified QDs with histidine tagged peptides and biomolecules.

6.1 Schematic showing the activity of NF-kB. (A) In unstimulated cell NF-kB is inactive as it is bound to inhibitory protein $\mathrm{IkB}$, (B) whereas in stimulated cell, proinflammatory stimuli activates specific protein kinase resulting in the degradation of IkB and nuclear translocation of NF-kB....................................... 103

6.2 Schematic of different methods and techniques for intracellular delivery of QDs (shown in red). 105

7.1 Confocal image of $\mathrm{H} 9 \mathrm{c} 2$ cells showing endocytosis like uptake of QDs (A) Phase contrast, (B) Fluorescence images of cells incubated at room temperature (RT) with QDs. The punctuate fluorescence is indicative of endocytic uptake pathway. The images are taken with $60 \mathrm{x}$ magnification 121

7.2 Confocal image of H9c2 cells showing blocking of endocytosis of QDs (A) Phase contrast, (B) Fluorescence images of cells incubated at $4^{\circ} \mathrm{C}$ with QDs. Minimal or very low fluorescence is observed indicating the successful blocking of endocytosis. The images are taken with $60 \mathrm{x}$ magnification 122

7.3 Confocal image of $\mathrm{H} 9 \mathrm{c} 2$ cells without QDs as negative control (A) Phase contrast, (B) Fluorescence images of cells incubated without QDs. Minimal or very low auto fluorescence from the cells is observed.

7.4 Reversible permeabilization using 1:1 hypotonic buffer ratio and cell viability. Comparison of bright field, fluorescence and viability representative images of H9c2 cells treated with 1:1 hypotonic buffer ratio for uptake of QDs for (A-C) 2 minutes, (D-F) 5 minutes and (G-I) 10 minutes respectively at 4oC. Bright field and fluorescence images are obtained with $60 \times$ magnification and cell viability images are obtained with $10 \times$ magnification. Cell viability fluorescence images $(\mathrm{C}, \mathrm{F}$ and $\mathrm{I})$ show fluorescence overlay of calcein AM (green: live cells) and ethidium homodimer-1 (red: cells with damaged cell membrane) .......................124

7.5 Reversible permeabilization using 3:2 hypotonic buffer ratio and cell viability. Comparison of bright field, fluorescence and viability representative images of $\mathrm{H} 9 \mathrm{c} 2$ cells treated with 1:1 hypotonic buffer ratio for uptake of QDs for $(\mathrm{A}-\mathrm{C}) 2$ minutes, (D-F) 5 minutes and (G-I) 10 minutes respectively at $4^{\circ} \mathrm{C}$. Bright field and fluorescence images are obtained with $60 \times$ magnification and cell viability images are obtained with $10 \times$ magnification. Cell viability fluorescence images $(\mathrm{C}, \mathrm{F}$ and $\mathrm{I})$ show fluorescence overlay of calcein AM (green: live cells) and ethidium homodimer-1 (red: cells with damaged cell membrane) 
7.6 Reversible permeabilization using 1:3 hypotonic buffer ratio and cell viability. Comparison of bright field, fluorescence and viability representative images of H9c2 cells treated with 1:1 hypotonic buffer ratio for uptake of QDs for (A-C) 2 minutes, (D-F) 5 minutes and (G-I) 10 minutes respectively at $4^{\circ} \mathrm{C}$. Bright field and fluorescence images are obtained with $60 \times$ magnification and cell viability images are obtained with $10 \times$ magnification. Cell viability fluorescence images $(\mathrm{C}, \mathrm{F}$ and $\mathrm{I})$ show fluorescence overlay of calcein AM (green: live cells) and ethidium homodimer-1 (red: cells with damaged cell membrane) ........................ 126

7.7 Live/Dead cell viability assay following hypotonic buffer treatment showing live cells stained with calcein AM (green: live cells) and dead cells stained with ethidium homodimer-1 (red: cells with damaged cell membrane). Cells are treated with different hypotonic buffer ratios $(1: 1,3: 2,1: 3)$ for different incubation times $(2,5$ and 10 minutes $)$ at $4{ }^{\circ} \mathrm{C}$ respectively........................................ 127

7.8 Quantitative analysis of internalized QDs with different hypotonic buffer ratios. Histograms represent mean fluorescence intensity of cells (arbitrary units) for 1:1 ratio $(12.24,17.62,20.31), 3: 2$ ratio $(10.65,18.37,18.96)$ and $1: 3$ ratio $(11.64$, $15.68,17.41$ ) for 2 minutes (blue), 5 minutes (green) and 10 minutes (red) respectively. The mean fluorescent intensity of cells without QDs used as negative control (gray) is 4.68 128

7.9 Reversible permeabilization using $25 \mu \mathrm{g} / \mathrm{ml}$ Saponin and cell viability. Comparison of bright field, fluorescence and viability representative images of $\mathrm{H} 9 \mathrm{C} 2$ cells treated with $25 \mu \mathrm{g} / \mathrm{ml}$ of Saponin for (A-C) 2 minutes, (D-F) 5 minutes and (G-I) 10 minutes respectively. Bright field and fluorescence images are obtained with $60 \times$ magnification and cell viability images were obtained with $10 \times$ magnification. Cell viability fluorescence images $(\mathrm{C}, \mathrm{F}$ and $\mathrm{I})$ show fluorescence overlay of calcein $\mathrm{AM}$ (green: live cells) and ethidium homodimer-1 (red: cells with damaged cell

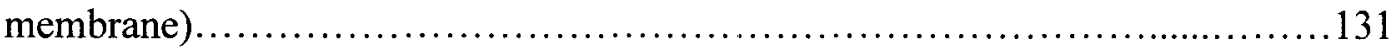

7.10 Reversible permeabilization using $50 \mu \mathrm{g} / \mathrm{ml}$ Saponin and cell viability. Comparison of bright field, fluorescence and viability representative images of H9C2 cells treated with $25 \mu \mathrm{g} / \mathrm{ml}$ of Saponin for (A-C) 2 minutes, (D-F) 5 minutes and (G-I) 10 minutes respectively. Bright field and fluorescence images are obtained with $60 \times$ magnification and cell viability images were obtained with $10 \times$ magnification. Cell viability fluorescence images ( $C, F$ and $I$ ) show fluorescence overlay of calcein AM (green: live cells) and ethidium homodimer-1 (red: cells with damaged cell

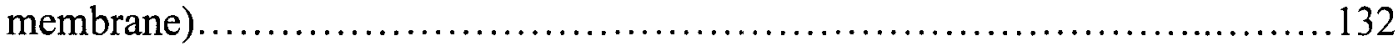

7.11 Reversible permeabilization using $75 \mu \mathrm{g} / \mathrm{ml}$ Saponin and cell viability. Comparison of bright field, fluorescence and viability representative images of $\mathrm{H} 9 \mathrm{C} 2$ cells treated with $25 \mu \mathrm{g} / \mathrm{ml}$ of Saponin for (A-C) 2 minutes, (D-F) 5 minutes and (G-I) 10 minutes respectively. Bright field and fluorescence images are obtained with $60 \times$ magnification and cell viability images were obtained with $10 \times$ magnification. Cell viability fluorescence images $(\mathrm{C}, \mathrm{F}$ and $\mathrm{I})$ show fluorescence overlay of calcein $\mathrm{AM}$ 
(green: live cells) and ethidium homodimer-1 (red: cells with damaged cell

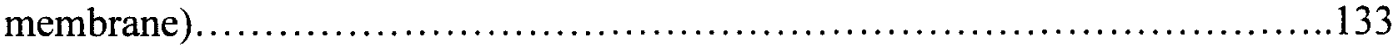

7.12 Live/Dead cell viability assay following Saponin treatment showing live cells stained with calcein AM (green: live cells) and dead cells stained with ethidium homodimer-1 (red: cells with damaged cell membrane). Cells are treated with different hypotonic (3:2) Saponin concentrations $(25 \mu \mathrm{g} / \mathrm{ml}, 50 \mu \mathrm{g} / \mathrm{ml}$ and $75 \mu \mathrm{g} / \mathrm{ml})$ for different incubation times $(2,5$ and 10 minutes $)$ at $4^{\circ} \mathrm{C}$ respectively 134

7.13 Quantitative analysis of internalized QDs with different Saponin concentrations. Histograms represent mean fluorescence intensity for $25 \mu \mathrm{g} / \mathrm{ml}(9.59,13.85$, $11.99), 50 \mu \mathrm{g} / \mathrm{ml}(10.21,16.43,19.38)$ and $75 \mu \mathrm{g} / \mathrm{ml}(13.32,16.97,14.03)$ for 2 minutes (blue), 5 minutes (green) and 10 minutes (red) respectively. The mean fluorescent intensity of cells without QDs used as negative control (gray) is 4.68

7.14 Reversible permeabilization using Saponin without hypotonic buffer conditions. (A) phase contrast, (B) fluorescence images of cells treated QDs mixed in $125 \mu \mathrm{g} / \mathrm{ml}$ of Saponin without hypotonic buffer................................... 137

7.15 Permeabilization using Saponin without hypotonic conditions. Comparison of bright field and fluorescence overlay images of calcein AM (green: live cells) and ethidium homodimer-1 (red: cells with damaged cell membrane) co-stained H9c2 cells treated with (A, B) $0 \mu \mathrm{g} / \mathrm{ml}$ Saponin, cells only (negative control), (C, D) $125 \mu \mathrm{g} / \mathrm{ml}$ Saponin without hypotonic buffer, (E, F) $1 \mathrm{mg} / \mathrm{ml}$ Saponin without hypotonic buffer (positive control) respectively

7.16 Quantitative analysis of live/dead cell assay performed on cells treated with Saponin without hypotonic buffer conditions. Comparison of percentage of live/dead cells stained with of calcein AM (green: live cells) and ethidium homodimer-1 (red: cells with damaged cell membrane) is shown for $0 \mu \mathrm{g} / \mathrm{ml}$ Saponin i.e., cells alone (negative control), $125 \mu \mathrm{g} / \mathrm{ml}$ Saponin without hypotonic buffer and $1 \mathrm{mg} / \mathrm{ml}$ Saponin without hypotonic buffer (positive control) respectively ............. 140

7.17 Gel electrophoresis data confirming the conjugation of QDs with NF-kB p65 antibodies. Tailing is due to the different number of antibodies attached to each QD.... 144

$7.18 \mathrm{Gel}$ electrophoresis data confirming the conjugation of QDs with protein $\mathrm{G}$ and with NF-kB p65 antibodies.

7.19 Stimulation of cells for activation and nuclear translocation of NF-kB protein. Immunofluorescence on cells stained for NF-kB protein using QDs: NF-kB p65 antibodies for (A) unstimulated cells, (B) UV radiation, (C) TNF- $\alpha$ and (D) LPS stimulated cells respectively. The arrows (white) are used to identify cells with 
nuclear fluorescence. The TNF- $\alpha$ stimulated cells are stained with QDs with $\sim 600$ $\mathrm{nm}$ emission. All the images are taken with a magnification of $60 \mathrm{x} \ldots \ldots \ldots \ldots \ldots 147$ 


\section{CHAPTER I - INTRODUCTION}

"The principles of physics, as far as I can

see, do not speak against the possibility of

maneuvering things atom by atom."

Richard Feynman, 1959. ${ }^{1}$

\subsection{INTRODUCTION}

Nanotechnology provides the ability to build molecular systems which can be manipulated atom by atom through the control over the structure of matter at the molecular level. Nobel Laureate Richard Feynman is credited for foreseeing the concept of nanotechnology in his talk "There is plenty of room at the bottom" in $1959 .{ }^{1}$

Nanomaterials are at the cutting edge of this rapidly developing technology. Traditionally, two complimentary approaches to nanomaterials are studied - the topdown approach, where the bulk material is machined down to the nanoscale and the bottom-up approach, building the material starting at the molecular or atomic level. Both these approaches are very widely used to create structures on the nanometer scale with the ability to manipulate at atom-by-atom precision. The fundamental properties of materials at the nanoscale are significantly different from the bulk. This is mainly due to the large fraction of surface atoms, high surface energy, spatial 
confinement and reduced defects. The properties of the nanomaterials like the size dependent physical and chemical properties, novel electronic and optical properties have been thoroughly studied. Now, the nanomaterials have entered into an application and commercialization exploration stage. ${ }^{2,3}$ In addition to the plethora of applications such as in electronics, optics and materials science, nanomaterials are very widely used in many biomedical applications. ${ }^{4-7}$ The relevant processes in many biological systems occur at the nanometer scale regime. Moreover, many of the elementary biological units such as DNA, proteins and cell membranes are in this nanoscale dimension. Therefore, nanomaterials are widely used in the biomedical field to better comprehend how the subcellular molecules, cells, tissues and organs function at fundamental level. This integration of the concepts of nanotechnology in biology and medicine is generally referred to as the field of nanomedicine. ${ }^{8}$

Nanomedicine broadly is defined as the technology that deals with the use of nanostructured materials as molecular tools for medical diagnosis and treatment. In recent years significant efforts are made toward understanding the molecular basis of diseases. ${ }^{9}$ As a consequence, new therapies have been developed to focus on targeting signalling at the cellular level. ${ }^{10} \mathrm{~A}$ major advancement in this area is the use of gene therapy to introduce, remove or silence genes within a cell. ${ }^{11}$ Delivery of genes or small interference RNA (siRNA) for gene silencing to the nucleus of the cells for gene therapy is challenging. Traditionally, the methods for delivery have been viral vector based approaches. Despite advantages like high transfection efficiency, high tropism and natural delivery method, viral methods have several disadvantages. The process itself is 
complicated and can result in chromosomal insertion, oncogene activation, strong immune reactions and contamination with live virus.

Novel nanomaterials like carbon nanotubes, colloidal crystals, magnetic nanoparticles and quantum dots are being studied for drug delivery applications. ${ }^{12-15}$ The selective delivery of drugs using targeted nanomaterials avoids unnecessary complications and increases the effectiveness of the drug delivered. Another major area for application of nanomaterials is cellular labelling and bio imaging. Semiconducting nanomaterials like single wall carbon nanotubes, nanoparticles and quantum dots possess superior optical properties than conventional labels. More recently, nanomaterials have been used to stimulate, and interact with target cells and tissues in controlled ways to induce desired physiological responses. ${ }^{16}$

\subsection{PURPOSE OF THE STUDY}

The purpose of this dissertation study is to demonstrate the intracellular delivery of nanomaterials for subcellular imaging and tracking of biomolecules. Specifically, two novel nanomaterials - single wall carbon nanotubes (SWCNTs) and colloidal quantum dots (QDs) are described.

Single walled carbon nanotubes (SWCNTs) have been widely used for many biomedical applications such as gene and biomolecular delivery applications. Though several in vitro and in vivo models are studied to evaluate the biocompatibility of the SWCNTs, their interactions with blood (specifically blood leukocytes) is not extensively investigated. In this dissertation, two possible interactions (direct and indirect) of SWCNTs with blood leukocytes are recreated in vitro and the response of the leukocytes 
to SWCNTs is thoroughly investigated. Despite the limitation of not being able to observe long term effects, this study provides a good starting point in trying to evaluate the activation of the blood leukocytes in response to SWCNTs.

Semiconducting nanoparticles or quantum dots (QDs) offer several advantages over traditional fluorescent molecules (organic dyes) such as high resistance to photobleaching and size tunable fluorescence emission making them excellent candidates for imaging and biomolecular delivery applications. However, a major challenge is to achieve freely dispersed QDs in live cells. Traditional methods such as endocytosis, lipids based delivery, electroporation are used which accomplish delivery of QDs into live cells as aggregates and trapped in vesicles. This limits the utility of the QDs for further labelling of other intracellular components or their specific targets. In this dissertation, efficient intracellular delivery of QDs in live cells is demonstrated using a novel reversible permeabilization technique. This approach combines the use of osmosis driven fluid transport into cells which is achieved by creating hypotonic environment and reversible permeabilization using low concentration of cell permeabilization agents like Saponin.

In this dissertation, Chapters II-IV deal with Carbon nanotubes while the chapters V-VII deal with quantum dots. Chapter II discusses the fundamental properties and the functionalization of SWCNTs for their use in biomedical applications. Chapter III discusses the novel approach developed in this dissertation to evaluate the interactions of SWCNTs with the (direct and indirect interactions) blood. The materials and methods used in this study are presented including the preparation of SWCNTs dispersions and isolation of peripheral mononuclear cells from the blood. Chapter IV presents the results 
obtained from direct and indirect interaction experiments of SWCNTs to blood leukocytes. Chapter $\mathrm{V}$ discusses colloidal quantum dots and the techniques used to conjugate QDs with various biomolecules. Chapter VI discusses the motivation behind the use of QDs in this dissertation. A novel technique developed in this dissertation based on the reversible permeabilization of live cells for the efficient delivery of QDs is described. Additionally, the potential of QDs: antibody (NF-kB p65) conjugates to label subcellular protein (NF-kB) is also discussed. Chapter VII presents the results obtained from the intracellular delivery of QDs using the reversible permeabilization technique. The activation, nuclear translocation and labelling of NF-kB protein using QDs: NF-kB p65 antibody conjugates is demonstrated. Chapter VIII provides the conclusions and potential future directions for research based on the results presented in this dissertation. 


\section{CHAPTER II -CARBON NANOTUBES}

\subsection{INTRODUCTION}

Carbon nanotubes (CNTs) are one dimensional nanomaterial made of carbon with a cylindrical nanostructure and with diameters of a few nanometers. Carbon nanotubes were first synthesized in 1991 at the NEC laboratories, Japan using an arc discharge evaporation method typically used for fullerene synthesis. ${ }^{17}$ Their unique properties have led to their use in various applications in nanotechnology ${ }^{18-20}$, electronics ${ }^{21-23}$, optics ${ }^{24,25}$ and materials science. ${ }^{26-28}$ More recently, they have been widely used in many applications in the biomedical field like delivery of therapeutics, ${ }^{29-31}$ cancer diagnostics, 32,33 and tissue engineering. ${ }^{34,35}$

This chapter briefly discusses the intriguing functional properties of the carbon nanotubes. The bio-functionalization of the CNTs and their biomedical applications are presented. An overview on the toxicity studies of the CNTs is also discussed. Finally, the use of CNTs in this dissertation research is given.

\subsubsection{Synthesis and properties}

CNTs are single or multiple cylindrical sheets of graphene. Therefore, they not only possess the inherent properties of their source graphite but also possess unique structural, electrical, mechanical, optical and biological properties. Primarily, they are classified based on the number of graphene sheets they are made of. When a single graphene sheet forms a nanotube, they are referred to as single walled carbon nanotubes 
(SWCNTs) whereas multiple sheets of graphene or multiple SWCNTs nested together coaxially are referred to as multi-walled carbon nanotubes (MWCNTs) ${ }^{36} \mathrm{~A}$ high resolution transmission electron microscope images of coaxial tubes of graphitic sheets is shown in Figure 2.1

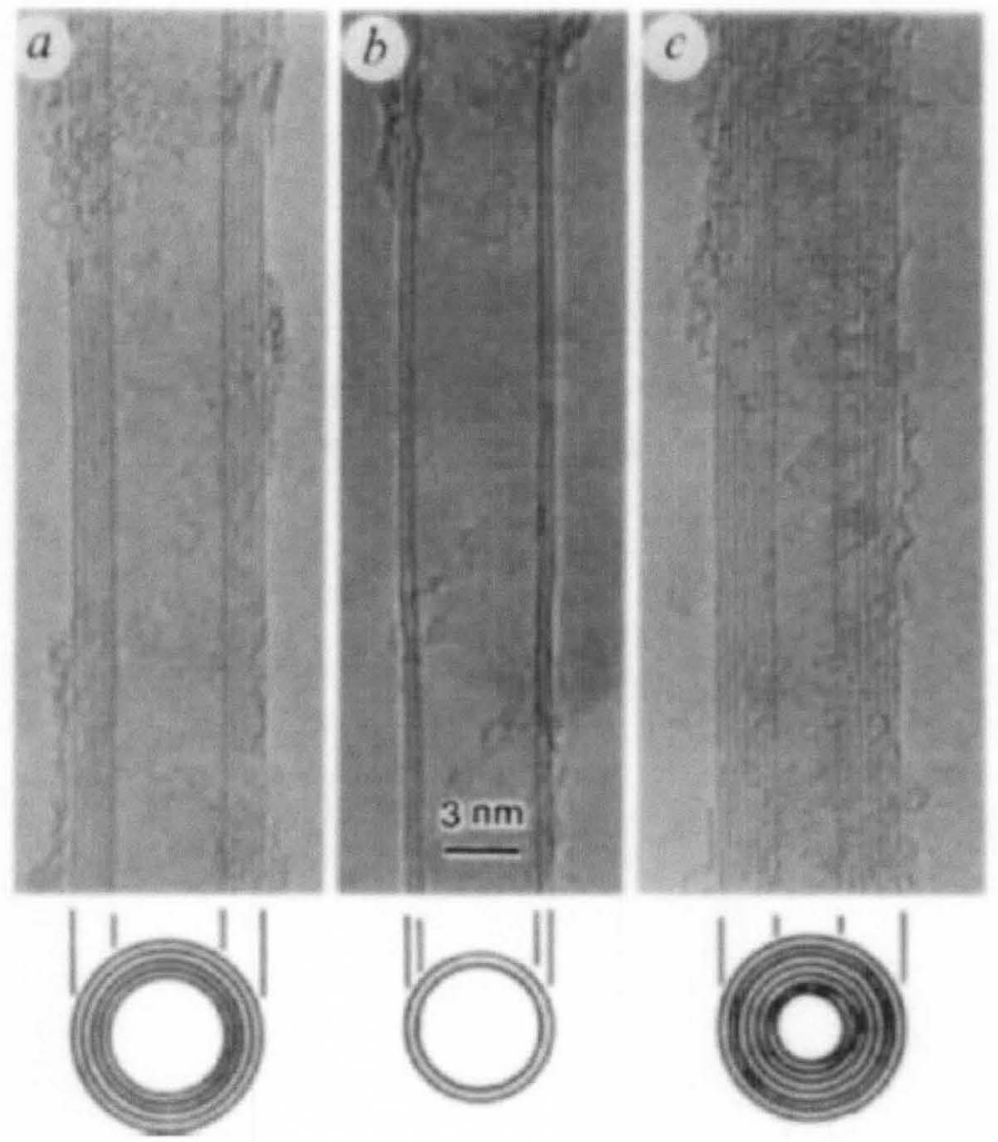

Figure 2.1 High resolution TEM images of MWCNTs formed by (a) five, (b) two, (c) seven graphene sheets. ${ }^{17}$

\section{Synthesis of CNTs}

Typically, CNTs are grown using three main methods- arc discharge, laser ablation and chemical vapor deposition (CVD) (which uses transition metal catalyst 
particles (Co, Fe, Ni) for the growth). ${ }^{37}$ In 1993, two seminal reports independently described SWCNTs synthesis using arc the discharge method in the presence of a metal catalyst. $^{38,39}$ The arc discharge method involves heating a doped graphite rod with an electric arc, and results in low purity due the amorphous carbon and low yield. Large quantities of MWCNTs ( $\sim$ in gram quantities) with diameters ranging from few nanometers to tens of micrometers have been synthesized using a variant of the arc discharge method. ${ }^{40}$ In 1996 , the synthesis of CNTs with high yield and purity was accomplished using a laser vaporization method developed by Nobel laureate Richard Smalley ${ }^{41}$ In the laser ablation method, a graphite target is vaporized using laser pulses in the presence of the catalyst particles. The nanotubes synthesized using the laser ablation method are of higher purity than those synthesize using the arc discharge method. CVD method for the synthesis of CNTs involves the decomposition of a carbon precursor gas over catalytic metal particles typically lying on a substrate. CVD growth of nanotubes is commonly used to produce highly pure CNTs with high yield. The most common precursor gases are used in the CVD synthesis of CNTs are methane, acetylene and ethylene.

\section{Properties of CNTs}

The fundamental physical, mechanical and electronic properties of CNTs are well reported and understood. This section gives a brief overview of the fundamental properties of CNTs with emphasis on the functional properties which are exploited for the use of CNTs in biomedical field. The excellent mechanical properties of the CNTs are due to the $\mathrm{sp}^{2}$ hybridized $\mathrm{C}-\mathrm{C}$ bonds. This results in very high Young's modulus ( 1000GPa) and tensile strength of about 50 times stronger than steel. The quantum 
confinement of the conduction band electrons, their nanoscale diameter with long lengths makes them excellent one-dimensional conductors. The electronic properties of the CNTs arise from the manner in which the underlying graphene sheet is rolled. A pair of indices $(\mathrm{n}, \mathrm{m})$ is used to denote the number of unit vectors along the two directions in the honeycomb lattice structure of the graphene sheet. Typically, the CNTs are specified in terms of diameter $(d)$ and the chiral angle $(\theta)$, and the chiral vector $\left(C_{h}\right)$. The chiral vector is defined in terms of the crystal lattice indices $(n, m)$ and the basic vectors $a_{1}$ and $a_{2}$ as $\mathrm{C}_{\mathrm{h}}=\mathrm{na}_{1}+\mathrm{ma}_{2}\left(\right.$ Figure 2.2). ${ }^{42-45}$

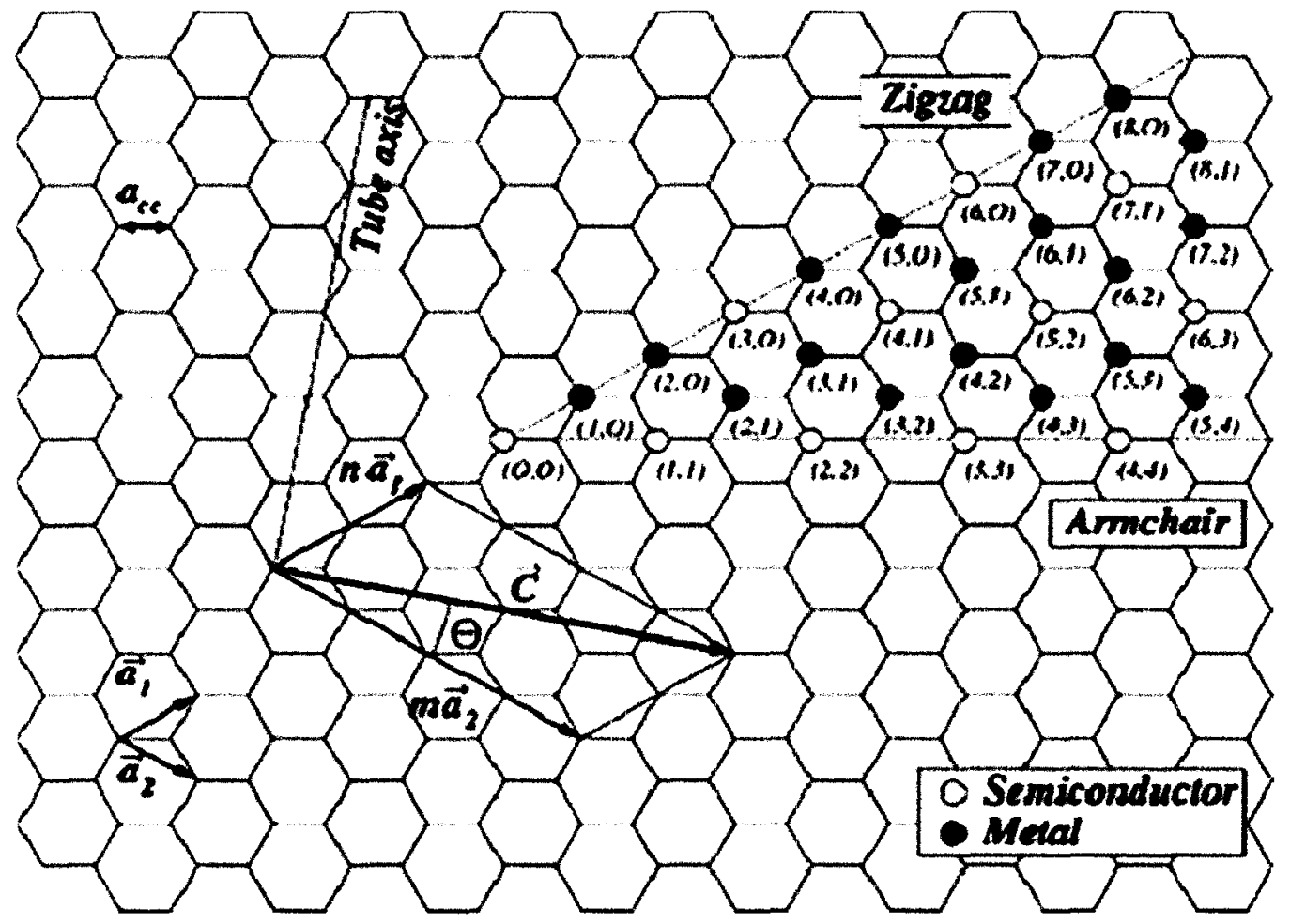

Figure 2.2 Diagram showing the 2D graphene sheet and the vector structure classification to define CNTs structure using the chiral indices $(n, m) .{ }^{42}$ Open circles represent semiconducting and solid circles represent metallic CNTs respectively. 
The values of the indices $(n, m)$ determine the chirality of the CNTs and a change in these parameters affects their electrical, optical and mechanical properties. Two types of CNTs exist depending on their electronic band structure. If the value of $n=m$, then the resultant nanotube is referred to as armchair nanotube. When $m=0$, it is called as a zigzag nanotube. Both the armchair and zigzag are collectively termed as achiral nanotubes. Any other values for the indices $(n, m)$ form a chiral nanotube. The diameter of the nanotube is defined by the formula:

$$
d=\frac{\left|\vec{C}_{h}\right|}{\pi}=\frac{a}{\pi} \sqrt{n^{2}+m^{2}+n m},
$$

where $a$ is the length of the unit vector which is approximately $2.46 \mathrm{~A}^{0}$. 46

Figure 2.2 shows the chiral indices $(n, m)$ of a range of nanotubes, with the open circles representing the semiconducting nanotubes and the solid circles representing metallic nanotubes. It is observed that armchair nanotubes are all metallic while one-third of zigzag and chiral nanotubes are metallic, and the rest are semiconducting. ${ }^{45}$ It is theoretically calculated that when $n=m$ (armchair tubes), the energy bands cross the Fermi level, giving rise to their metallic nature. ${ }^{43}$ Also it is predicted that when $n-m=3$ $x \mathrm{~L}$ ( $\mathrm{L}$ is an integer), then the CNTs are metallic in nature, and when $\mathbf{n}-\mathrm{m} \neq 3 \times \mathrm{L}$, then the tubes formed are semiconducting in nature with an energy gap in the order of approximately $0.5 \mathrm{eV}$. These theoretical predictions are confirmed by experiments that measure the density of states (DOS) of the CNTs (Figure 2.3). The DOS of the CNTs have a number of sharp peaks or van-Hove singularities each of which corresponds to a quantum sub band. The energy gaps of the semiconducting CNTs is inversely proportional to the diameter of the tubes and at fairly large diameters, the energy gap approaches to zero, i.e., to that of the graphene sheet itself. As shown in the Figure 2.3, it 
can be seen that the first energy gap is wider for metallic tubes than that of semiconducting tubes. As the energy is increased, van-Hove singularities appear at specific energy levels.

\subsection{CNTS IN THE BIOMEDICAL FIELD}

\subsubsection{Bio-functionalization}

The most attractive property of the CNTs which is widely exploited in the biomedical field is their ability to be functionalized with various biomolecules resulting in their integration in a variety of applications. The following section discusses the various methods used to functionalize CNTs for biological applications.

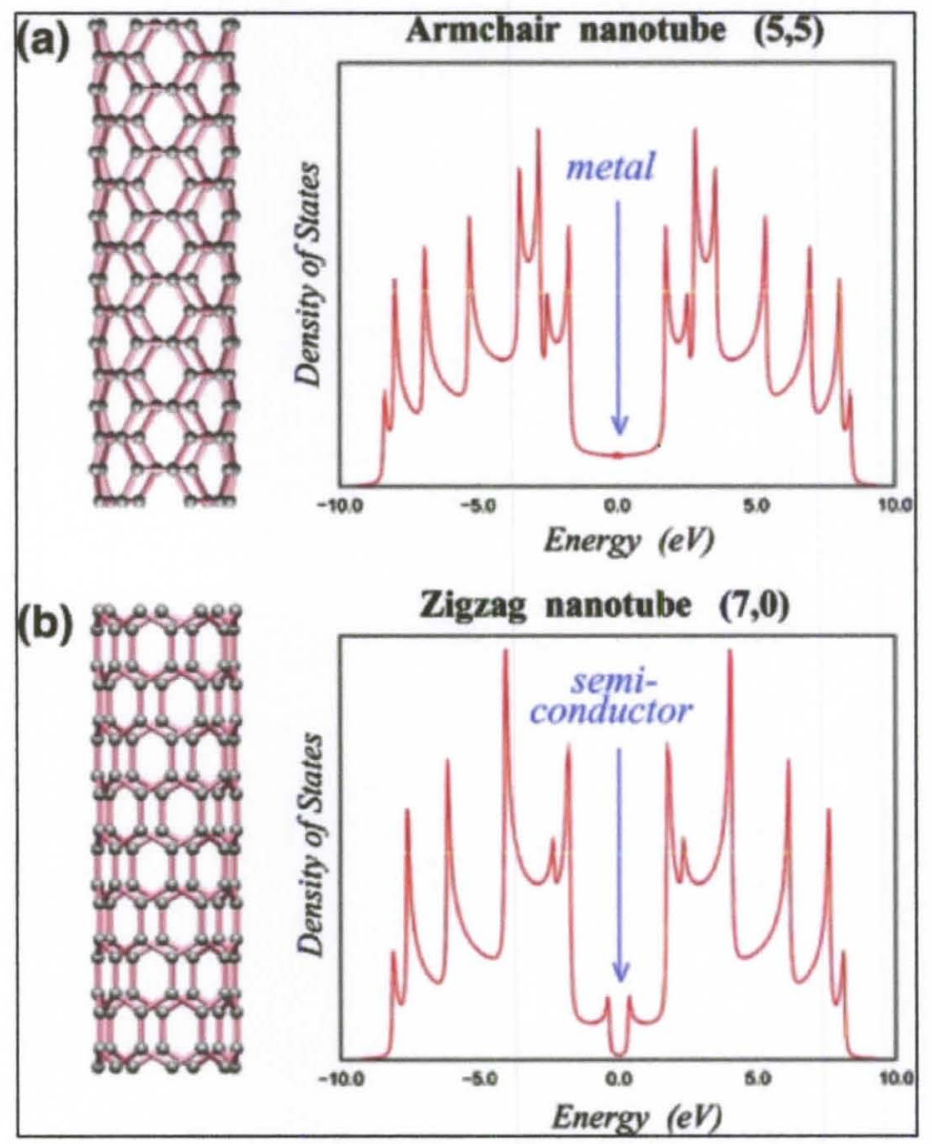

Figure 2.3 Density of states of (a) armchair CNT $(5,5)$ exhibiting metallic character and

(b) zigzag $\mathrm{CNT}(7,0)$ with semiconducting in nature. ${ }^{47}$ 
The high surface area, varied electronic properties, and excellent chemical and thermal stability has led to CNTs being used for many biological applications. ${ }^{48,}{ }^{49}$ The major difficulty in the use of CNTs in bio-applications is their poor solubility and polydispersity in aqueous and non-aqueous solvents. Chemical modification of CNTs has been studied extensively to efficiently functionalize them with various functional moieties. ${ }^{50-53} \mathrm{~A}$ number of methods have been developed for bio-functionalization of CNTs with proteins, nucleic acids and various other biomolecules. ${ }^{54}$ Some of the most commonly used methods are discussed here.

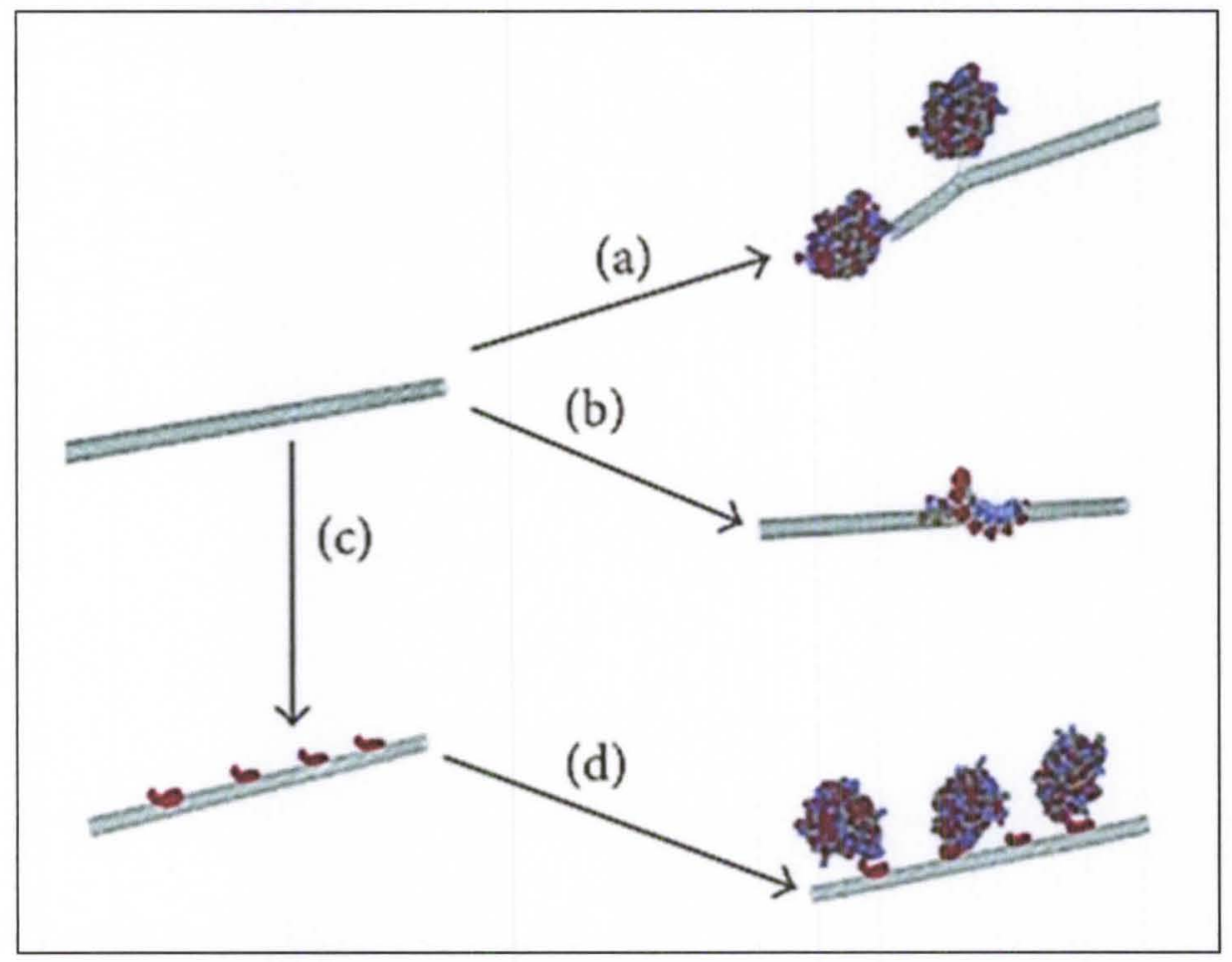

Figure 2.4 Bio-functionalization of CNTs using (a) covalent functionalization, (b) noncovalent interactions, (c, d) hybrid approach. ${ }^{54}$

The methods of CNTs bio-functionalization are classified (depending on the nature of the interactions of biomolecule to the CNTs) into covalent functionalization, non-covalent 
functionalization and hybrid approaches (Figure 2.4). The most common method for covalent functionalization of CNTs involves two steps. In the first step, the CNTs are oxidized using strong acids such as $\mathrm{HNO}_{3}$ and $\mathrm{H}_{2} \mathrm{SO}_{4}$ which results in the formation of carboxyl groups (-COOH) at the open ends and defect sites on the surface. ${ }^{55}$ In the next step, biomolecules containing reactive amine groups are covalently attached to the carboxyl groups on the CNTs surface. Though the covalent functionalization of CNTs is one of the simplest approaches, it is the least specific and excessive oxidation may lead to the damage of the structure of the CNTs (Figure 2.4a).

The large aromatic ( $\pi$-electrons) hydrophobic surface of CNTs makes them excellent candidates for non-covalent interactions with suitable biomolecules. The hydrophobic interactions are strong and can be utilized to attach various biomolecules (Figure 2.4b). The interactions can also take place inside the CNTs; however, their small internal diameter (1-2 $\mathrm{nm})$ limits the use of internal functionalization. A hybrid approach for the bio-functionalization of CNTs involving both the non-covalent and covalent interactions for CNTs bio-functionalization also exists. In the first step, an 'anchor' molecule is attached to the CNTs using the non-covalent interaction method. In the second step, a chemical reaction links the anchor molecule to the biomolecule of interest. (Figure 2.4c, d). The non-covalent method as well as the hybrid method does not involve any chemical modification of the surface, thus the intrinsic structural properties of the CNTs are preserved. However, the non-covalent approach can result in nonspecific binding of molecules to the CNTs.

In this dissertation, SWCNTs are bio-functionalized with DNA using the noncovalent approach. The use of non-covalent interactions between DNA and CNTs was 
first reported by Tsang et al and Guo et al. ${ }^{56,57}$ They functionalized CNTs with platinated and iodinated helical double stranded DNA and used them as probes for visualization of DNA. Zheng et al. functionalized SWCNTs with single stranded DNA (ss-DNA) using a simple sonication and centrifugation procedure. ${ }^{58}$ Molecular simulations revealed that the ss-DNA helically wraps the SWCNTs and separates them, thus avoiding aggregation of the nanotubes. As shown in Figure 2.5,the hydrophobic nucleic acid bases (red) stack on the surface of the nanotubes while the sugar phosphate backbones of the nucleic acids point outward from the sidewall into the aqueous environment. The binding of ss-DNA with the SWCNTs competes quite effectively with that of the tube-tube interactions.

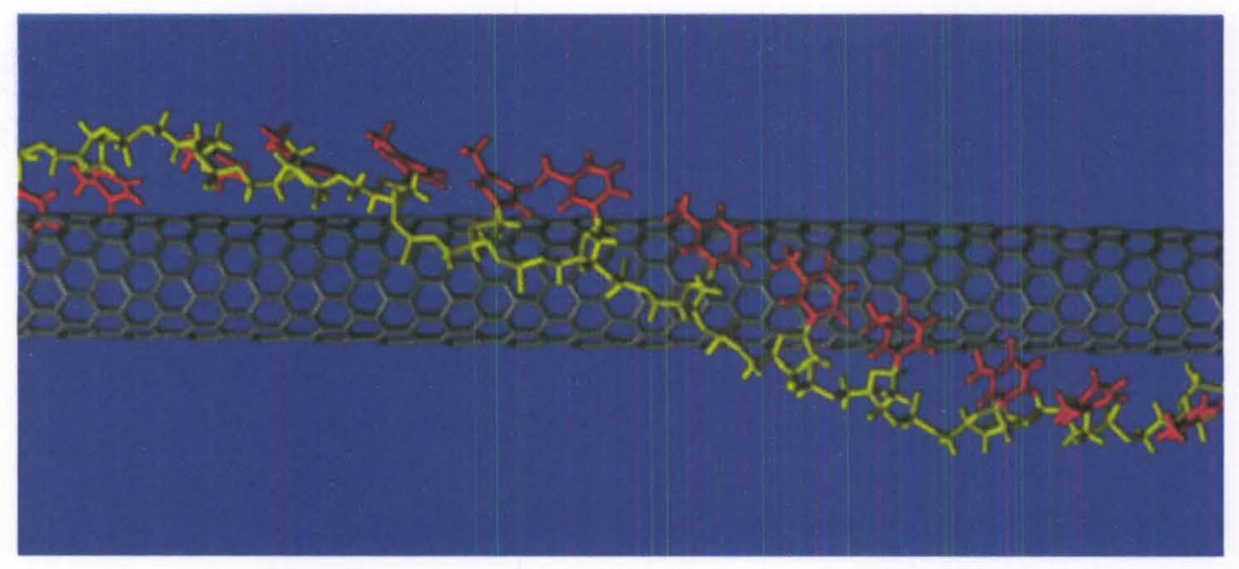

Figure 2.5 Simulation showing the wrapping of DNA on a SWCNT. The nuclei acid bases are color coded red while the sugar phosphate backbone is depicted in yellow. ${ }^{58}$

\subsubsection{Biological applications}

The bio-functionalization of the CNTs has led to their use in many biological applications including drug delivery, biological sensing, and medical diagnostics. ${ }^{29,} 59$ Some of the recent and more popular biological applications are discussed in this section. 
CNTs are of particular interest in gene and drug delivery due to their ability to be functionalized with various biomolecules and transported along with their cargo across the cell membrane. ${ }^{60,61}$ Physical and biomolecular functionalization appear to be critical determinants of trans membrane transport. ${ }^{60}$ One study in particular has demonstrated that the length of CNTs $(\sim 200 \mathrm{~nm})$ is an important determinant of preferential uptake in mammalian cells. ${ }^{62}$ Functionalized SWCNTs were used as molecular transporters of short interfering RNA (siRNA) into human T cells and primary cells and silenced the expression of HIV-specific cell-surface receptors and co-receptors. ${ }^{63}$ Chemically functionalized MWCNTs were used as substrate for the culturing and controlling the growth of the neurons. ${ }^{64}$ The SWCNTs functionalized with double stranded DNA and SWNTs were used for glucose sensing applications. ${ }^{65}$ Semiconducting SWCNTs show strong absorption and fluorescence emission in the near infrared region. ${ }^{66}$ They are used as long term optical labels in cells due to their extremely photo stable fluorescence emission in the biological transparent near infrared wavelengths. ${ }^{67}$ The optical absorption of SWCNTs in the biologically transparent window $(700-1000 \mathrm{~nm})$ was utilized for selective cancer cells destruction ${ }^{68}$. Once SWCNTs are internalized into cells or tissues, they function as small antennas which absorb laser radiation light that is transferred to the cells in the form of heat energy, thus triggering cell death. In a similar study, Gannon et al. exploited the near infra-red absorption of SWCNTs functionalized with a biocompatible polymer for enhanced destruction of cancer cells using non-invasive radiofrequency field. ${ }^{69}$

Despite establishment of the fact that various mammalian cells uptake SWCNTs readily, the exact mechanism of uptake is still not well understood. Endocytosis is widely 
speculated as the method for internalization for both covalently and non-covalently modified $\mathrm{CNTs}^{70}$ while phagocytosis is also considered as a potential pathway. ${ }^{71}$ In addition to these cellular processes, passive insertion of the nanotubes modified with hydrophilic terminus into the cell membrane is also demonstrated.$^{30}$ Recent reports also indicate that SWCNTs transport across the cell membrane is not affected in the presence of endocytosis inhibitors like sodium azide or reduced temperature suggesting the existence of several different and energy independent pathways. ${ }^{60}$

\section{$\underline{2.3 \text { TOXICITY STUDIES }}$}

Before the results and findings from cell culture-based CNTs studies can be translated to in vivo patient models, the overall effect of CNTs at the cellular level and the consequence of these events at the systemic level must be thoroughly evaluated. Several studies have looked at the toxicity of CNTs using cellular-level models. Magrez et al. investigated the toxicity of carbon based nanomaterials and concluded that acid treatment of CNTs led to increased toxicity. ${ }^{72}$ The toxicity of acid treated MWCNTs was also reported by Bottini and co-workers who concluded that pristine nanotubes are less toxic than their acid treated counterparts. ${ }^{73}$ Monteiro et.al reported that chemically unmodified MWCNTs localize within the cytoplasmic vacuoles of human epidermal cells and initiate an irritation response. ${ }^{74}$ Pulskamp et al. reported that the toxicity of CNTs could be a result of the metal traces associated with the growth processes. ${ }^{75}$ Though there are several reports which suggest the potential toxicity of CNTs, there are several other reports which claim that CNTs are non-toxic. SWCNTs were functionalized with fluorescence markers(FITC) and peptides and were used to deliver these molecules into cell without any apparent toxicity. ${ }^{76}$ Dumortier et al. reported that functionalized and 
highly water soluble CNTs are non-cytotoxic and preserve the functionality of primary immune cells. ${ }^{77}$ Another study performed by Cui et al. investigated the effect of SWCNTs on human embryo kidney cells (HEK) and reported that high concentrations of SWCNTs inhibited cell proliferation while lower concentrations has minimal or very slight influence on the cells. ${ }^{78}$ It was observed that CoMoCAT nanotubes were not inherently cytotoxic to HeLa cells. The interaction of SWCNTs with human epithelial like cells was investigated by Yehia et al. and it was observed that the SWCNTs dispersions are inherently non-toxic to cells. ${ }^{79}$ Thus the results from the cell culture studies seem to vary depending on the cell type, experimental conditions as well as the dosage and functionalization of the CNTs.

Several in vivo studies on the toxicity of CNTs were reported. Lam et al. studied the potential pulmonary toxicity in mice and observed that high doses induced lung lesions while low doses do not show any overt clinical effects. ${ }^{80}$ Muller et al. investigated the respiratory toxicity of MWCNTs in rats and reported that the nanotubes induced inflammatory and fibrotic reactions even after 60 days. ${ }^{81}$ Poland et al. compared the MWCNTs to asbestos, whose fibers were responsible for a worldwide pandemic of lung diseases. ${ }^{82}$ MWCNTs with diameters around $15-150 \mathrm{~nm}$ and lengths from 5-50 $\mu \mathrm{m}$ were used to investigate their toxicity in mesothelial lining of mice. Similar to asbestos, a length dependent pathogenic behavior was seen, with the observation of lesions and inflammations. The acute and chronic toxicity of functionalized SWCNTs injected into the bloodstream of mice was investigated by Gambhir et al. ${ }^{83}$ Though it was observed that SWCNTs persisted in the liver and spleen for 4 months, no cytotoxicity was observed. More recently, Bai et al. reported that repeated administrations of water soluble 
MWCNTs in mice results in reversible testis damage repairable within 90 days without any loss of fertility. ${ }^{84}$ Though there are several reports, no consensus still exists on the toxicity of CNTS. An appropriate model needs to be established to thoroughly evaluate the effects of CNTs on the human body.

\subsection{CNTS IN THIS DISSERTATION}

In this dissertation, blood is chosen as the appropriate tissue culture model. Blood perfuses throughout the human body and its cellular populations can potentially interact with SWCNTs functionalized with ss-DNA. In this dissertation, SWCNTs grown using CoMoCAT method (Cobalt, Molybdenum catalyst) are used. As purchased SWCNTs are non-covalently functionalized with ss-DNA and dispersed in aqueous solutions for biocompatibility. The toxicity of the SWCNTs is studied by evaluating their interactions with blood cells especially leukocytes (white blood cells) and investigating the response of leukocytes to SWCNTs.

The next chapter discusses the experimental materials and methods used in this dissertation to evaluate the toxicity of SWCNTs. The method used to disperse the SWCNTs and the methods employed to study the response of blood to SWCNTs are discussed in detail. 


\section{CHAPTER III - INTERACTION OF SWCNTS WITH BLOOD LEUCKOCYTES}

\subsection{INTRODUCTION}

Carbon nanotubes can be functionalized with various biomolecules like proteins and nucleic acids for gene and drug delivery applications. However, the overall effect of CNTs on the body at the cellular and systemic level is not well understood. Hence, interaction of the CNTs with cells for potential toxicity and unnecessary inflammation needs to be carefully evaluated.

Several studies have been performed to study the response of CNTs using cell culture ${ }^{74,85-87}$ and animal models. ${ }^{84,88,89}$ However, the results vary depending on the cell type, functionalization of the CNTs and experimental conditions. Even though animal models provide some understanding of the response of body to CNTs, the translation of those observations to human studies is not straightforward. The response of human immune system to CNTs is significantly more challenging and has not been extensively investigated. In this dissertation, the interaction of CNTs with the human immune system was evaluated by investigating the response of immune cells in the blood to CNTs.

Blood is a living tissue that perfuses the entire body and contains cellular populations that could potentially interact with CNTs. Hence, evaluation of the different cellular populations (specifically leukocytes) in blood can potentially provide vital information regarding the inflammatory response to the CNTs. Blood 
samples were isolated from healthy donors to study the interactions of the blood leukocytes with CNTs. This in vitro study mimics critical events in vivo. The initial sections of this chapter deal with the basics and the cells involved in the immune response of the body. The materials and methods used in evaluating the interaction of CNTs to blood leukocytes are discussed at the end of the chapter.

\subsection{IMMUNE RESPONSE IN HUMAN BODY}

The resistance of the human body to infections is defined as the immunity of the body. The cells and biomolecules that help in maintaining this resistance constitute the immune system of the body. The collective reaction of these cells and molecules to infections and infectious diseases is the immune response of the body. Thus immunology is the study of the body's defense against infection. The basic functions of the immune system are:-

1. Recognition of the disease causing agents like the bacteria, viruses, microbes and other pathogens as well as toxins, chemicals, drugs and any other foreign particles.

2. Removal of the infection causing agents by performing immune effector functions.

3. Ability to self-regulate without unnecessary immune response and

4. Immunological memory.

The fundamental function of the immune system is to protect the body from any potential infectious agents by recognizing specific antigens. Antigens are typically receptor molecules on the surfaces of the cells, viruses, fungi and bacteria but substances like toxins, chemicals, drugs or any other foreign objects may also act as antigens. Once the 
antigens are recognized, specialized cells and molecules perform effector functions in order to remove the infectious agents. An important function of the immune system is the ability to not respond to the host's own antigens (immunological tolerance). This selftolerance is extremely important in applications like tissue grafts or transplantation experiments. For example, when tissues are transplanted from one individual to another individual who are not identical twins, the donor cells are treated as foreign and destroyed by the recipient's immune system. Here, the antigens on the donor cells generate immune response even in the absence of any infection. However, when a neonatal mouse is transplanted with cells from a mouse of different strain, it was observed that some of these cells survived for most of the recipient's life time. In this case, the immune system is unresponsive to foreign antigens and is known as acquired immunological tolerance. In a similar way, immune system acquires tolerance to its own macromolecules which is known as natural immunological tolerance. The breakdown of this tolerance to self-antigens leads to autoimmune diseases and the mechanism leading to this breakdown are relatively unknown.

Another major function of the immune system is to remember prior experiences with antigens. The immune system creates immunological memory following initial exposure to antigens. ${ }^{90}$ This memory leads to quicker and enhanced response to subsequent encounters with the same antigen. This memory helps the body develop immunity towards many common infectious diseases and it is how vaccinations confer long lasting protection against infections. Typically, the organs in the peripheral lymphoid system contain three types of cells - naïve cells, effector cells and memory cells. During the initial encounter with an antigen, naïve cells respond by proliferation and differentiation 
into effector cells which carry out the immune response. At the same time, some of the naïve cells differentiate into long living memory cells. During the subsequent exposures to the same antigen, the memory cells respond more quickly than the naïve cells in creating effector cells to fight the antigen. The memory cells can either differentiate into effector cells or more memory cells. These memory cells can sometimes live for the lifetime of an individual providing lifelong immunological memory. A process known as clonal expansion is responsible for the creation of memory cells from the naïve cells (Figure 3.1).

\subsection{CELLS IN THE IMMUNE SYSTEM}

This section deals with the various cells involved in the immune responses in the body (specifically blood leukocytes). The immune system consists of the lymphoid tissues and organs of the body. Lymphoid tissues are concentrated majorly in the bone marrow, lymph nodes, spleen, liver, thymus, and as Peyer's patches scattered in the linings of the gastrointestinal tract. The distribution of lymphatic tissues that make up the immune system in the human body is illustrated in the Figure 3.2.

The cells of the immune system originate from a pluripotent hematopoietic stem cell in the bone marrow. They are divided into two major lineages - myeloid progenitor cell and lymphoid progenitor cells (Figure 3.3). ${ }^{91}$ The myeloid cells include monocytes, macrophages, dendritic cells, meagakaryocytes and granulocytes (cells with the presence of cytoplasmic granules) while the lymphoid cells consists of T cells, B cells and natural killer (NK) cells respectively. In the event of hematopoiesis, cells in the bone marrow develop into either mature cells or precursors of cells that migrate out of the bone marrow. The bone marrow produces lymphocytes (B-cells, immature T-cells, and NK 
cells), granulocytes (including neutrophils, monocytes and dendritic cells), along with red blood cells (RBCs) and platelets. The immature $\mathrm{T}$ cells leave the bone marrow and migrate to the thymus where they mature and get released into the blood stream.

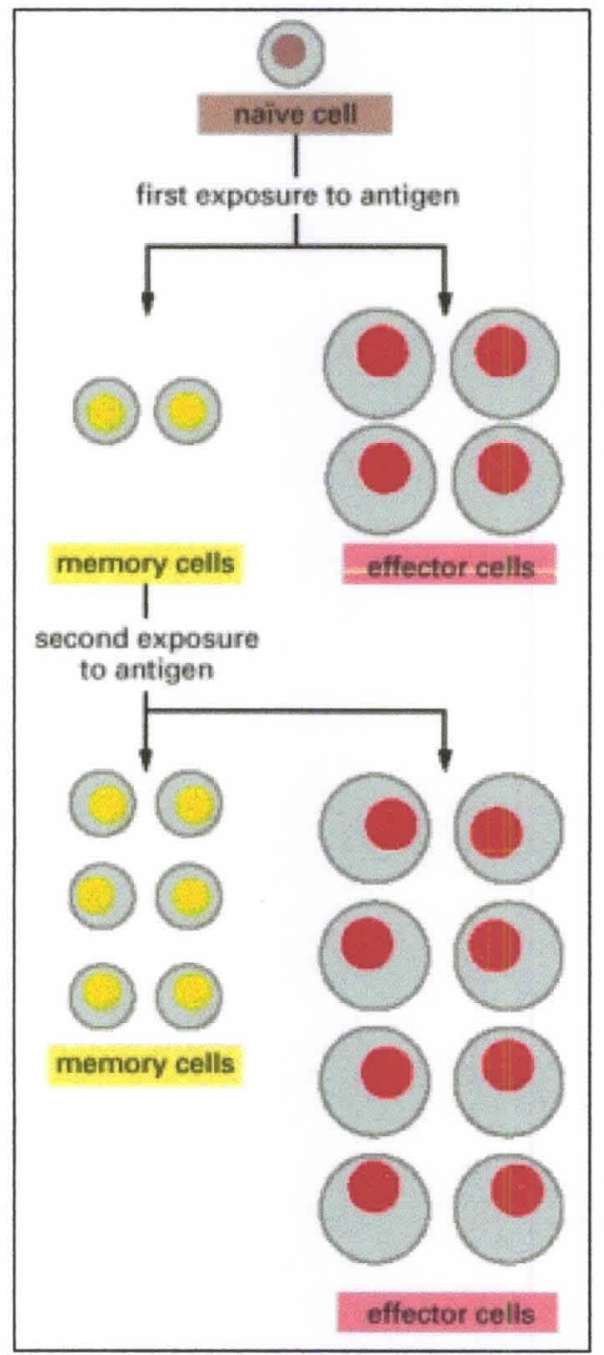

Figure 3.1 Schematic representation of immunological memory function of immune system. $^{92}$

The lymphatic system is a part of the circulatory system that consists of lymphatic vessels carrying a clear fluid called lymph towards the heart. Lymph nodes are an 
important part of the immune system and are found throughout the body that are mainly composed of T cells, B cells, dendritic cells and macrophages. The spleen consist of T cells, macrophages, dendritic cells, NK cells and RBCs and is involved in filtering antigens directly from the blood. The cells of the immune system are mainly divided into lymphocytes, specialized cells that capture and display the antigens called antigen presenting cells (APCs) and effector cells that destroy the antigens (Figure 3.4). The important functional properties of these major cell populations are discussed in the following sections.

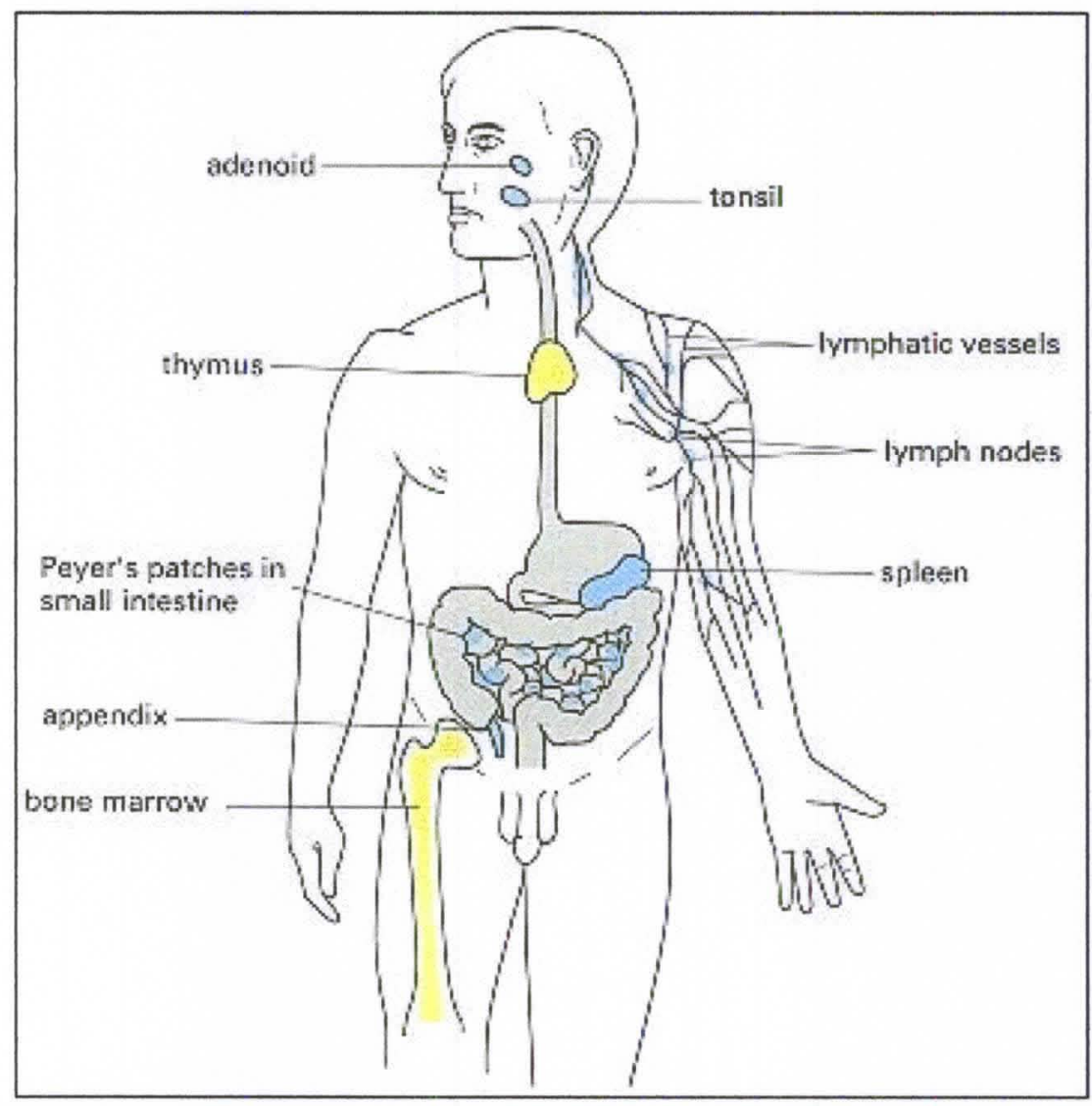

Figure 3.2 Anatomy of the major human lymphoid organs. The thymus and bone marrow (yellow) form the primary lymphoid organs. The other organs (blue) form the peripheral or the secondary lymphoid organs. ${ }^{92}$ 


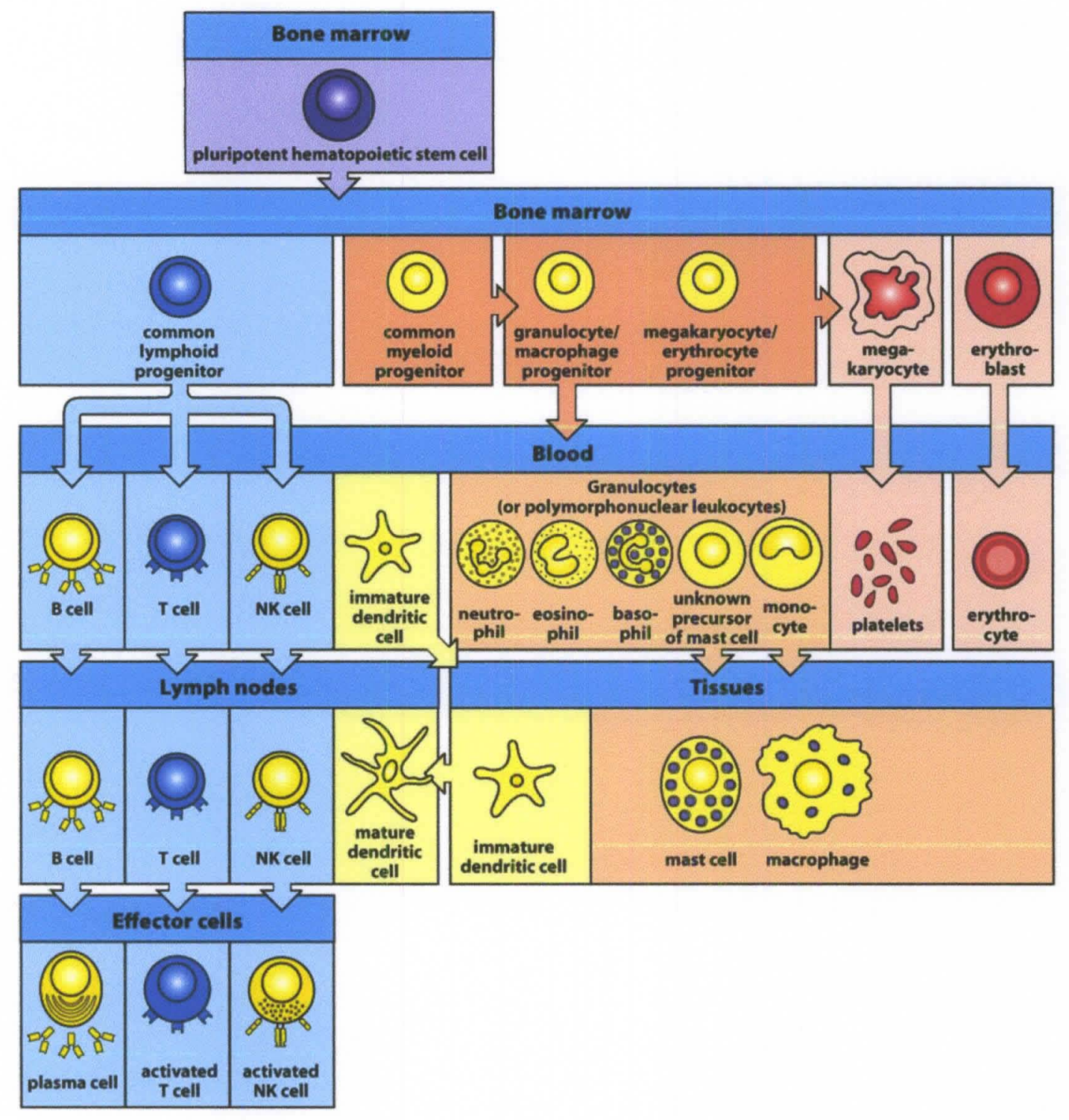

Figure 3.3 Cells of the immune system derive from precursors in the bone marrow (pluripotent hematopoietic stem cell). ${ }^{91}$

\subsubsection{Lymphocytes}

Lymphocytes are a type of blood leukocytes and are very large in number in the human body $\left(\sim 2 \times 10^{12}\right)$ which makes the immune system comparable to the liver or 
brain in cell mass. Even though they look morphologically similar, the lymphocytes are highly heterogeneous in lineage, function and phenotype. They are the only specialized cells with specific receptors which can recognize various antigens. Lymphocytes are distinguished by the proteins on their surface which are typically identified by panels of monoclonal antibodies. A standard nomenclature for these cell surface proteins is the cluster of differentiation (CD). CD is used to delineate the proteins that define a specific cell type or cell differentiation stage which are recognized by a cluster of antibodies. Typically, the cellular populations are defined using a ' + ' or a '-' symbol to indicate whether a certain population expresses or lacks a $\mathrm{CD}$ molecule. For example, a " $\mathrm{CD}^{+} /$ CD4" cell is one that expresses CD3, but not CD4. Lymphocytes can be divided into large lymphocytes such as natural killer (NK) cells and small lymphocytes like T cells and B cells. A brief description of these lymphocytes is presented in the following section.

\section{$\underline{\text { Natural killer (NK) cells }}$}

NK cells constitute about $10 \%$ of the lymphocytes in the blood and peripheral lymphoid organs. These cells express specific surface molecules which respond to intracellular antigens by destroying the infected cells. The infected cells are differentiated from the normal cells by recognizing the changes in the surface molecules such as MHC (major histocompatibility complex) class I. NK cells respond to a variety of cell-signaling protein molecules called cytokines resulting in their activation. Once activated, they release cytotoxic (cell-killing) granules which can create holes in the plasma membrane of the infected cells, thereby destroying them. Another major function of activated NK 
cells is to synthesize and secrete cytokines that activates other specialized cells like macrophages to become more effective in killing the phagocytosed antigens.

\begin{tabular}{|l|l|}
\hline Cell type & Principal function(s) \\
\hline $\begin{array}{l}\text { Lymphocytes: B lymphocytes; } \\
\text { Tlymphocytes; natural } \\
\text { killer cells }\end{array}$ & $\begin{array}{l}\text { Specific recognition of antigens: } \\
\text { B lymphocytes: mediators of humoral } \\
\text { immunity } \\
\text { T lymphocytes: mediators of cell-mediated } \\
\text { immunity } \\
\text { Natural killer cells: cells of innate immunity }\end{array}$ \\
\hline \begin{tabular}{|l|l|} 
Antigen-presenting cells: \\
dendritic cells; macrophages; \\
follicular dendritic cells
\end{tabular} & $\begin{array}{l}\text { Capture of antigens for display } \\
\text { to lymphocytes: } \\
\text { Dendritic cells: initiation of T cell responses } \\
\text { Macrophages: initiation and effector phase } \\
\text { of cell-mediated immunity } \\
\text { Follicular dendritic cells: display of antigens } \\
\text { to B lymphocytes in humoral immune } \\
\text { responses }\end{array}$ \\
\hline $\begin{array}{c}\text { Effector cells: T lymphocytes; } \\
\text { macrophages; granulocytes }\end{array}$ & $\begin{array}{l}\text { Elimination of antigens: } \\
\text { T lymphocytes: helper T cells and cytotoxic } \\
\text { Tlymphocytes } \\
\text { Macrophages and monocytes: cells of the } \\
\text { mononuclear-phagocyte system } \\
\text { Granulocytes: neutrophils, eosinophils }\end{array}$ \\
\hline
\end{tabular}

Figure 3.4 The fundamental cells of the immune system. The major cells that mediate immune responses along with their functions are shown. Microscopy images of some cells are shown in the left panel. ${ }^{93}$ 


\section{T lymphocytes ( $T$ cells)}

$\mathrm{T}$ lymphocytes ( $\mathrm{T}$ cells) develop in the thymus and hence their name is derived based on the organs in which they develop. Based on their phenotype and functionality, they are divided into two major subsets- $\mathrm{T}$ helper $\left(\mathrm{T}_{\mathrm{h}}\right)$ cells and cytotoxic $\mathrm{T}$ (Tc or CTL) cells. Mature $T_{h}$ cells express the surface protein $C D 4$ and are referred to as $C D 4^{+} T$ cells. $\mathrm{T}_{\mathrm{h}}$ cells coordinate and regulate immune responses by mediating the secretion of lymphokines or stimulate other immune cells. Hence, the $T_{h}$ cells are called helper cells as they help other specialized lymphocytes (B cells) to produce antibodies and help phagocytes (cell which can engulf antigens) to destroy ingested microbes. The second type of T lymphocytes is cytotoxic T lymphocytes (Tc cells or CTLs). They are called as cytotoxic $\mathrm{T}$ cells because they lyse the cells which contain intracellular microbes. Cytotoxic T cells are involved in directly killing certain cancer cells, virus-infected cells and also some parasites. They are also known as protein $\mathrm{CD}^{+} \mathrm{T}$ cells as they express that particular protein. These cells are also important in down-regulation of immune responses. Both the $T_{h}$ as well as $T_{c}$ cells are located throughout the body in the lymph nodes and the spleen, liver, lungs, the intestinal tract and also in the blood.

\section{B lymphocytes ( $B$ cells)}

The lymphocytes that develop in the bone marrow are referred to as B lymphocytes (B cells). B cells are the only cells which can produce antibodies to fight infection. The specialized proteins that can recognize and bind to specific antigens that caused their activation are known as antibodies. Following stimulation by foreign antigens like bacteria, viruses and infected cells, B cells develop into antibody secreting plasma cells. B cells not only express antibodies that serve as receptors for antigens but 
also help initiating activation of cells. In addition to differentiating into plasma cells, some B cells develop into immune memory cells.

The different types of lymphocytes are shown in the Figure 3.5. The development and activation of both $\mathrm{T}$ cells and B cells is shown in the Figure 3.6. Both T and B cells which originate from the pluripotent hemopoietic stem cells give rise to all of the blood cells, including RBCs, white blood cells, and platelets. ${ }^{92}$

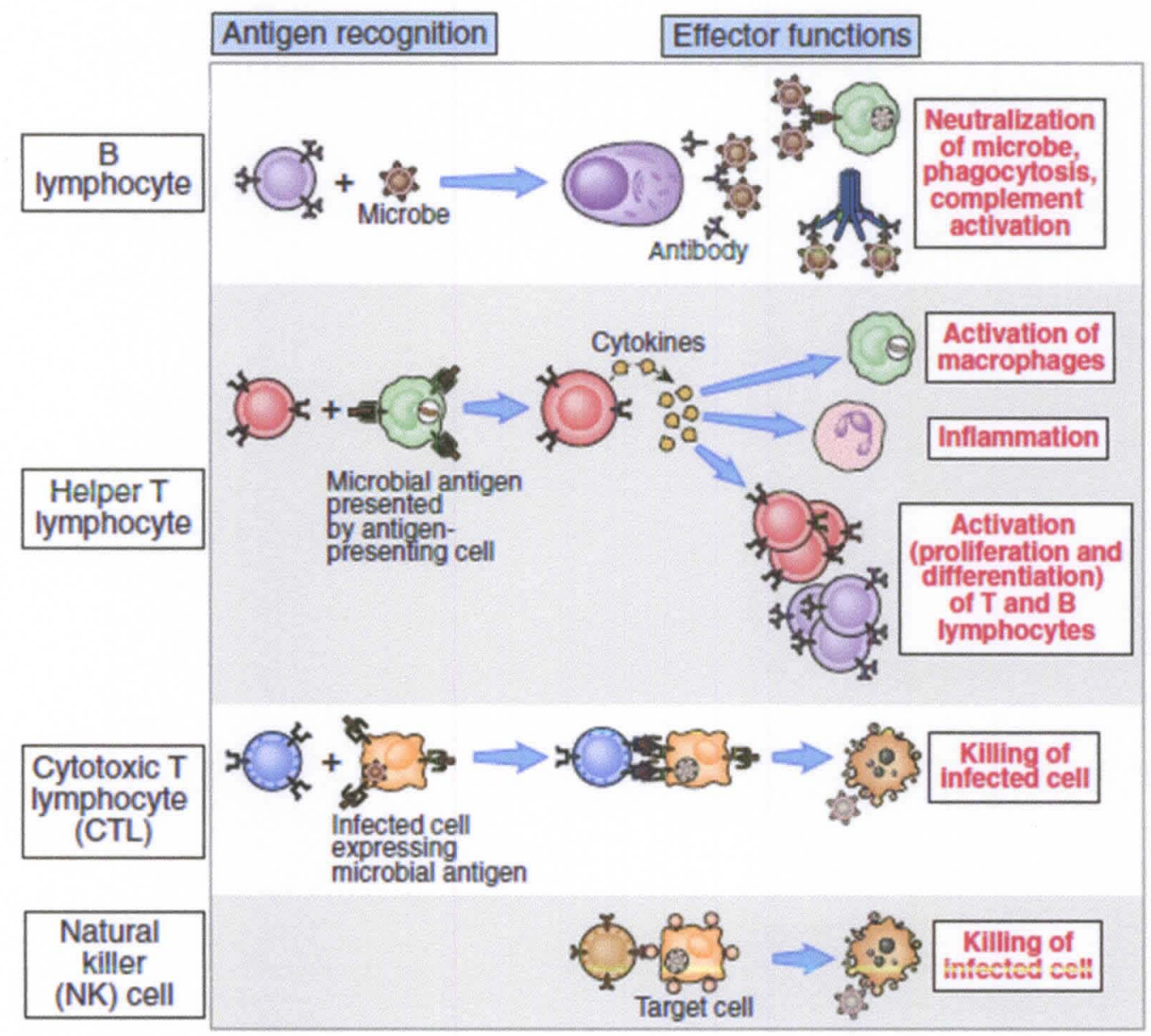

Figure 3.5 The different types of lymphocytes. Different classes of lymphocytes recognize antigens and differentiate into effector cells which help remove the antigens. B cells secrete antibodies to fight infection. $\mathrm{T}$ helper cells recognize antigens on the 
surfaces of APCs and secrete cytokines. Cytotoxic T cells directly kill the infected cells. NK cells recognize infected cells via receptors and kill the infected cells. ${ }^{93}$

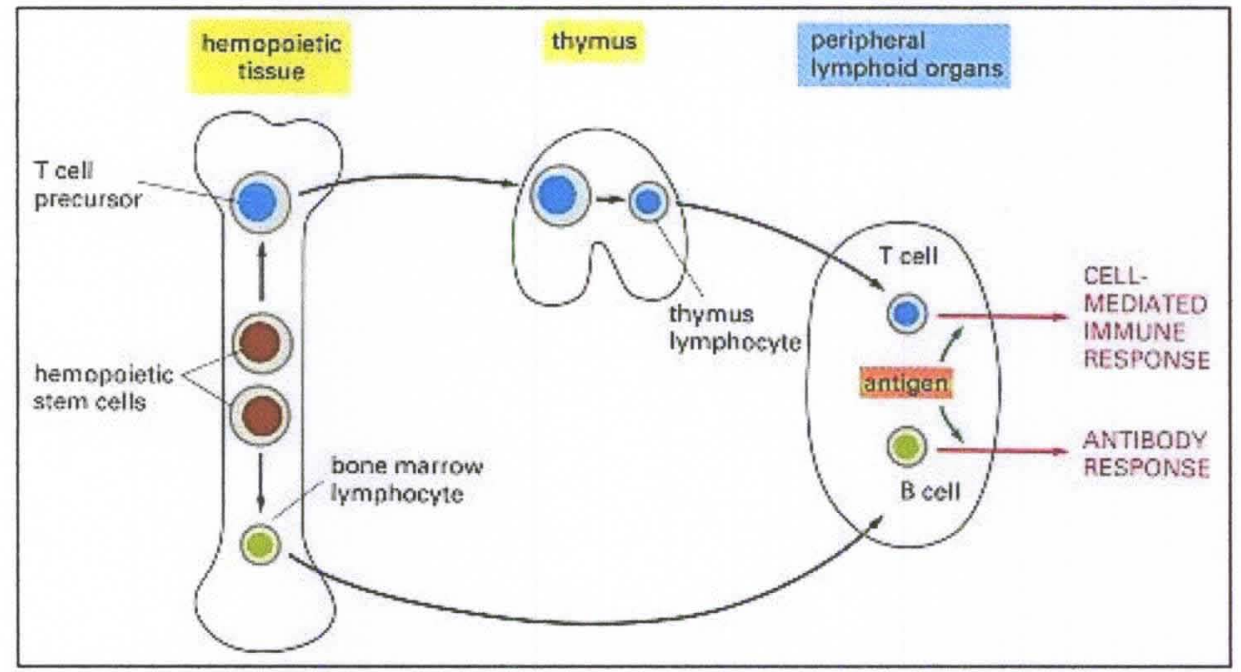

Figure 3.6 Schematic showing the development and activation of T cells and B cells. ${ }^{92}$

\subsubsection{Antigen presenting cells (APCs)}

The second major class of immune cells is the antigen presenting cells or APCs. Pathogens and microbes enter the body through the skin, gastrointestinal tract or the respiratory tract. The specialized cells present in the epithelium capture the antigens and transport them to peripheral lymphoid tissues. Here, the captured antigens are displayed to T cells; hence these specialized cells are referred to as antigen presenting cells (APCs). $\mathrm{T}$ cells can only recognize antigens when they are processed and presented by cells via MHC class I and II carrier molecules. Dendritic cells and macrophages typically function as APCs. Dendritic cells are present in the tissues which are in contact with external environment such as the skin, epithelia, inner lining of nose and lung as well as in the blood in immature state. Macrophages are white blood cells which are derived from the monocytes in the bone marrow. Macrophages phagocytose the antigens which escape 
through the epithelium and display the protein antigens to T cells. The APCs not only display the antigens to $\mathrm{T}$ cells but also have the ability to trigger the $\mathrm{T}$ cell immune responses. Such APCs are referred to as professional antigen presenting cells (APCs). The professional APCs respond to the antigens by first internalizing them via phagocytosis. Then, they produce surface proteins that activate the naive $\mathrm{T}$ cells by providing an additional co-stimulatory signal for $\mathrm{T}$ cell proliferation and differentiation. A special type of dendritic cells called the follicular dendritic cells which exist in the germinal centers of the lymphoid follicles display the antigens to B cells thereby stimulating B cell differentiation.

\subsubsection{Effector Cells}

The third major cells in the immune system are the effector cells whose function is primarily to eliminate the foreign antigens or microbes. The effector cells are mainly constituted by lymphocytes and leukocytes like macrophages and granulocytes. The basic functions of lymphocytes ( $\mathrm{T}$ cells and B cells) are discussed in the previous section.

In this section, circulating blood leukocytes are discussed. There are mainly two types of circulating phagocytes- neutrophils and monocytes that are recruited to the sites

of infection. Neutrophils or polymorphonuclear leukocytes (PMNs) are a subset of granulocytes and are the most abundant leukocytes in the blood with approximately 4000 to 10,000 cells per $\mathrm{mm}^{3}$.They serve as the first responders to most of the bacterial and fungal infections. In the event of infections, production of neutrophils from the bone marrow increases rapidly, sometimes to 20,000 per $\mathrm{mm}^{3}$. Their production is usually mediated by cytokines which are produced by many cells in response to any infections. Monocytes are typically low in number $\left(\sim 500-1000\right.$ per $\left.\mathrm{mm}^{3}\right)$ in the blood. They ingest 
microbes or foreign antigens present in the blood and can differentiate into macrophages and dendritic cells in the tissues. Macrophages are activated owing to an immune response and travel to the site of the infection. The recognition of the pathogens or microbes by the macrophages results in the phagocytosis of the antigens as well as activation of the phagocytes to kill the ingested microbes.

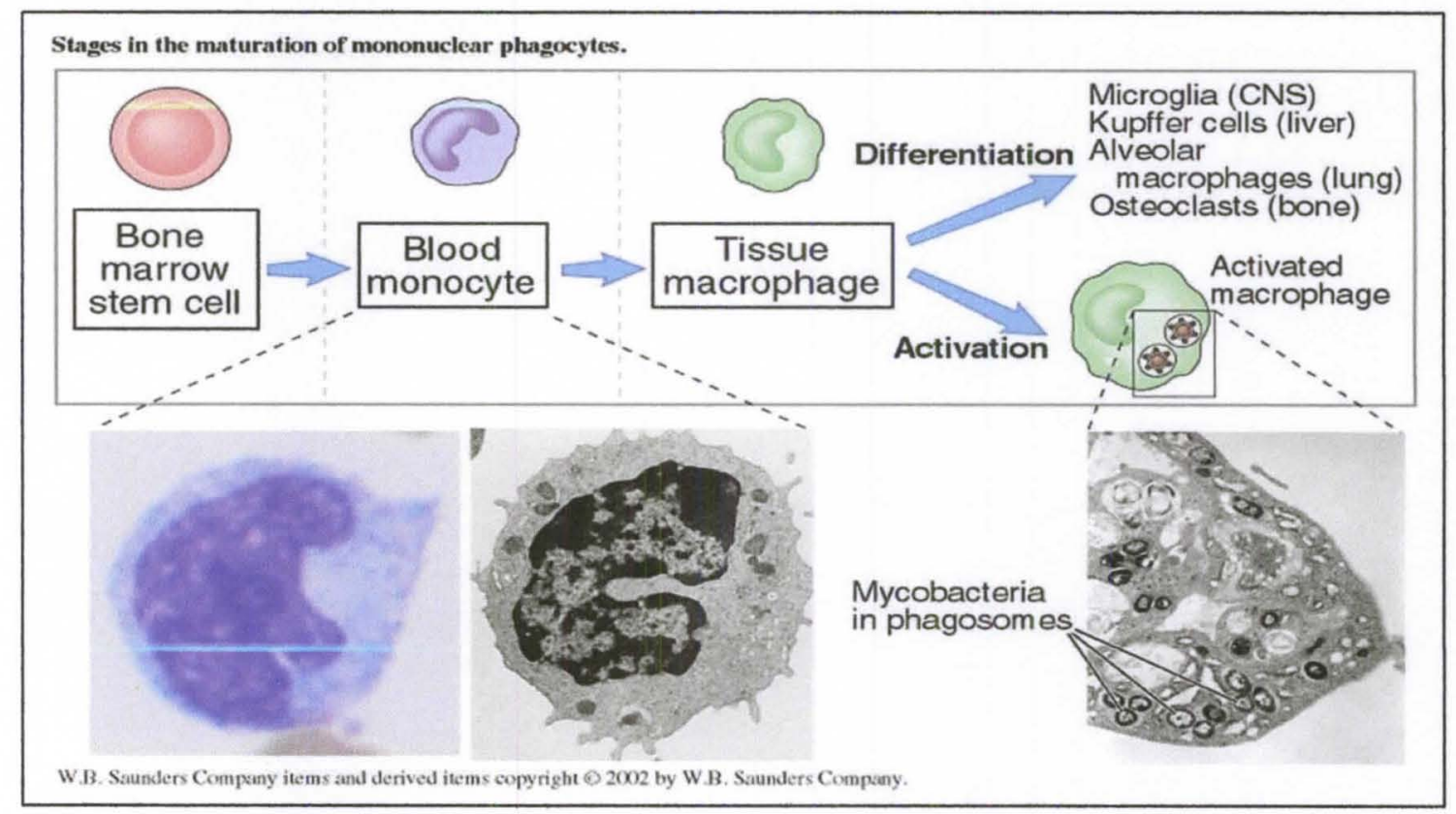

Figure 3.7 Stages in the maturation of mononuclear phagocytes. ${ }^{93}$ Monocytes are derived from bone marrow stem cells differentiate into macrophages in tissues. Macrophages may be activated in the tissues in response to different antigens and may differentiate into specialized forms in different tissues. A light and an electron microscopy image of the macrophage is shown in the lower left panel. An electron microscopy image of an activated macrophage is shown in the lower right panel.

When a macrophage ingests a pathogen, the pathogen is sequestered in the intracellular food vacuole, which later fuses with a lysosome. A variety of intracellular enzymes and toxic peroxides ingest the pathogen. Macrophages secrete interferons, 
lysozyme and other useful factors which activate lymphocytes and other immune cells to respond to the pathogens. Both neutrophils and monocytes migrate to the site of the infections and bind to the endothelial adhesion molecules. The migration of leukocytes to the extravascular infection site occurs within a few minutes. This migration process follows a sequence of events. Briefly, first these cells roll on the endothelium with the help of adhesion molecules called selectins; then they are adhered firmly using another set of adhesion molecules called as integrins. Finally chemoattractant cytokines also known as chemokines are produced that activate and stimulate the migration through the endothelium to the site of the infection.

Thus the lymphocytes, antigen presenting cells (APCs) and the effector cells are all involved in the immune response in the body.

\subsection{TYPES OF IMMUNE RESPONSE}

The immune responses of the body are typically divided into two main categoriesinnate immunity and adaptive immunity, which are discussed in the following section.

\subsubsection{Innate Immunity}

Innate immunity is the native or natural immunity an individual is born with. It is the initial host's defense to prevent the entry of infectious agents and rapidly eliminate the ones which enter. It is an antigen nonspecific defensive response of the body to any infection or antigen. The first line of defense in this type of response is provided by the skin and the other epithelial barriers. These barriers prevent the entry of any potential pathogens acting as a physical barrier between the inside of the body and the outside. The inner surfaces of the epithelial barriers are coated with mucus layers which are made of mucin and other glycoproteins. These layers protect the body not only from microbes 
from attaching to the epithelium but also from physical or chemical infections. Low molecular weight peptides called defensins are found in the lung and gastrointestinal tracts. They possess the ability to fight gram-negative and gram-positive bacteria, fungi, parasites and viruses. Chemical agents like fatty acids in the sweat and the lysozyme, phospholipase in tears and saliva help in the prevention of bacterial growth and destabilization of bacterial membranes. In the event of infectious agents breaching these physical barriers, specialized cells such as phagocytes, NK cells and various plasma proteins recognize and react against the infection. As described in the previous section, phagocytes like macrophages and neutrophils ingest and kill the pathogens. Thus, the epithelium, cell in the circulation and tissues and other plasma proteins constitute the components of the innate immunity.

Innate immunity is divided into immediate innate immunity and early induced innate immunity. Immediate innate immunity typically starts $0-4$ hours following exposure to an infectious agent and is carried out by circulating anti-microbial enzymes and proteins in the blood and other anatomical barriers. Early induced innate immunity typically begins anytime between 4- 96 hours after exposure to an infection and involves the recruitment of phagocytes like neutrophils, monocytes, macrophages and NK cells.

\subsubsection{Adaptive immunity}

Adaptive immunity also known as acquired immunity is the response of the host to stimulation by antigens and other pathogens that infect the tissues. The adaptive immunity is carried out mainly by lymphocytes and the antibodies produced by them. The lymphocytes express the receptors that are specific to different antigens and so the adaptive immunity is antigen specific immune response. Adaptive immune response 
takes a long time to develop, sometimes even several days and one develops this immunity throughout life. There are two types of adaptive immunity - humoral immunity and cell-mediated immunity. Humoral immunity involves the mediation of antibodies produced by B cells. The primary function of the antibodies is to prevent the foreign antigens present in the blood from gaining access to host cells and tissues. However, antibodies do not provide protection from intracellular antigens or antigens that live and divide inside infected host cells. Cell-mediated immunity protects the host from such intracellular antigens and it is mediated by the specialized $\mathrm{T}$ cells. A schematic of the fundamental mechanisms involved in both innate and adaptive responses is shown in Figure 3.8.

The most important properties of adaptive immune responses are

1. Specificity.

2. Memory

3. Specialization and

4. Nonreactivity to self-antigens.

Unlike innate immunity, adaptive immune responses possess the ability to recognize and respond to many different antigens. The immune system can potentially distinguish millions of different antigens in a highly specific manner. This remarkable specificity is due to the fact that the lymphocytes involved in this immunity consists of many different clones each of which expresses an antigen receptor that is different from other clones. This type of response is predicted by clonal selection theory which states that clones of lymphocytes specific to different antigens are created before even being exposed to these 
antigens. Therefore an antigen activates only those lymphocyte clones that are already committed to respond to it.

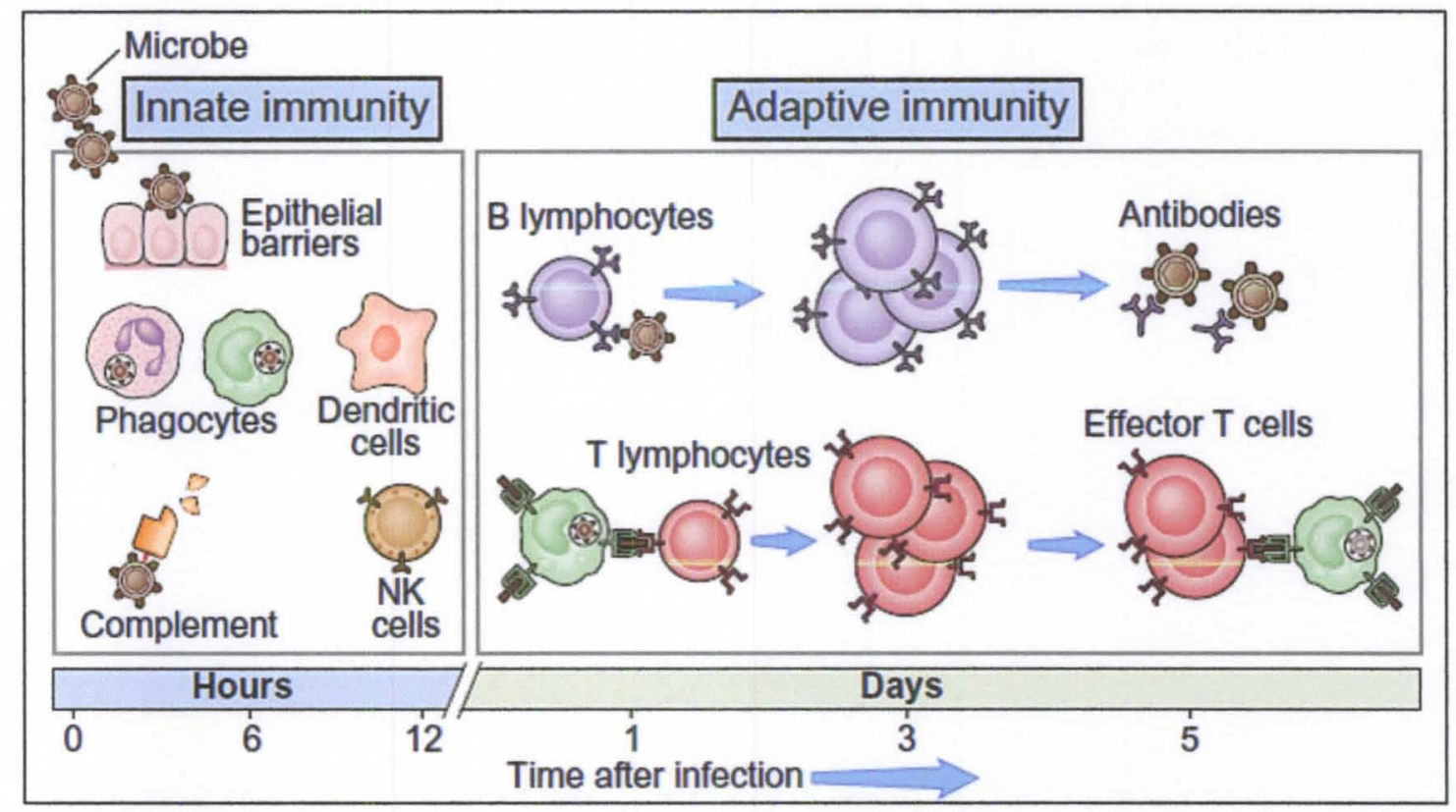

Figure 3.8 Schematic of the fundamental mechanisms involved in innate and adaptive immunity. The kinetics of the innate and adaptive immune response varies depending on the type of infection. ${ }^{93}$

According to this theory, the immune system functions on the principle of readmade response rather than made-to-order response. As described in the previous sections, repeated exposure to the same antigen results in larger and more effective immune responses. The primary immune response to the first exposure to an antigen is usually mediated by naïve lymphocytes while the subsequent exposures to the same antigens are mediated by memory lymphocytes. This memory response is usually quick, larger and more efficient in eliminating the antigens than the primary response. Thus the adaptive 
immune response with its immunological memory provides the body the ability to fight recurrent infections more efficiently. The specialization property of the adaptive immune response refers to the optimization of the immune response to different antigens. Adaptive immunity usually improves upon repeated exposure to a given antigen or infection. Another vital property of the adaptive immunity is the nonreactivity to selfantigens which prevents immune responses against host cells and tissues.

It is important to note that both the innate and adaptive immune responses work together to protect the host against any foreign infections. Lymphocytes are the major contributors in adaptive immunity; however they require the activation of innate immunity to respond to foreign antigens. APCs like dendritic cells and macrophages display the foreign microbes or antigens to the $\mathrm{T}$ cells and initiate an adaptive immune response (Figure 3.9).

\subsection{EVALUATION OF IMMUNE RESPONSE TO CNTS}

The previous sections provide a background of the immune response of the body to foreign antigens. This section deals with the evaluation of immune response of the body to CNTs. In order to understand the systemic effects of CNTs to the body, blood was chosen as an appropriate model system. Blood consists of many of the immune cells that perfuse the entire body; therefore analysis of cells in blood especially blood leukocytes provides a unique opportunity to understand inflammation at the both the cellular and systemic levels. 


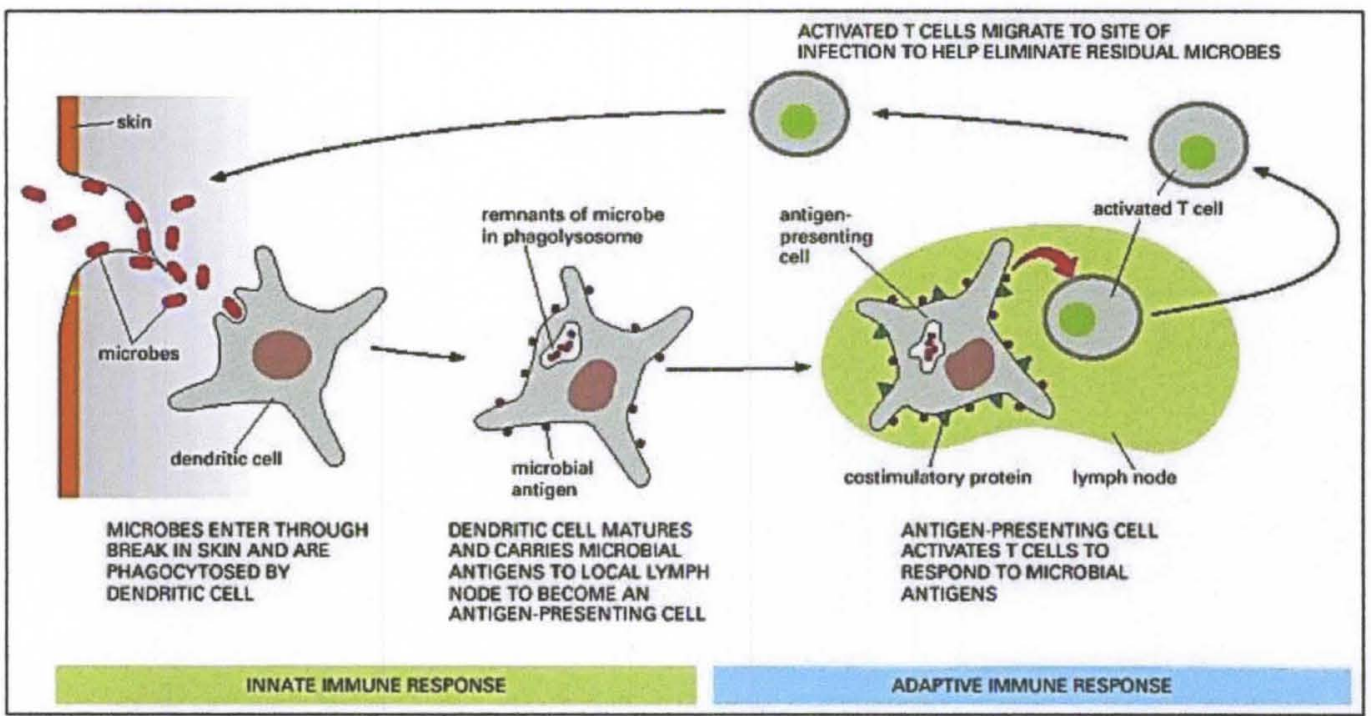

Figure 3.9 Innate immune response helps in the activation of adaptive immune response. $^{92}$

In this dissertation, acute response of the blood to CNTs is performed by extracting blood samples and stimulating the samples in vitro. This study attempts to replicate the acute response of blood leukocytes directly to CNTs analogous to innate immune response and indirectly via APCs which is analogous to adaptive immune response. The CNTs used in this study are functionalized with single stranded DNA (ssDNA) fragments composed of Guanine-Thymine (GT) repeat sequences. The ss-DNA acts as a surfactant to ensure dispersion and prevent aggregation of the CNTs as well as has the potential to be further modified with other biomolecules. Two possible interaction events are recreated which mimic the potential interactions of biomolecules via CNTs in the body. The first event, a direct interaction event recreates the presence of the CNTs in the blood circulation while the second event, an indirect interaction event recreates the activation of the different leukocytes in the blood via APCs with internalized CNTs. 
These two events provide an in vitro alternative to evaluate the acute immune response of blood leukocytes to CNTs.

\subsection{MATERIALS AND METHODS}

The following sections discuss the materials and methods and the analysis procedures for assessing the response of different leukocyte populations to CNTs.

\subsubsection{Materials}

Carbon nanotubes (CNTs) synthesized using CoMoCAT (Cobalt Molybdenum catalyst) methods are purchased from Southwest nanotechnologies (Norman, OK). The carbon nanotubes used in this dissertation are single walled carbon nanotubes and hence are referred to as SWCNTs from this point forward. Single stranded DNA (ss-DNA) with repeating Guanine-Thymine (GT) $)_{30}$ is purchased from Operon biotechnologies (Huntsville, AL). The size of the ss-DNA is 30 bases (alternating $\mathrm{G}-\mathrm{T}$ bases) and formula weight is around $10,000 \mathrm{Da}$. The ss-DNA is fluorescently labeled with a fluorescence dye molecule (FAM-Q) at one of its ends which is used to image the ssDNA inside the cells. Phosphate buffered saline (PBS), 1\% HAB (Hank's balanced salt solution without calcium or magnesium also containing $1 \%$ bovine serum albumin, $0.1 \%$ sodium azide, and $1 \mathrm{mM}$ ethylenediamine tetraacetic acid), Tris-buffered ammonium chloride buffer for RBCs lysis, RPMI-1640 cell culture media (ATCC 30-2001) and ficoll-paque solution are purchased from Sigma Aldrich( St. Louis, MO). Trypsin EDTA (Mediatech, Manassas, VA) is used to detach the cells from the surface for flow cytometry studies (BD FACS calibur). The blood leukocytes are stimulated for their activation using phorbol 12-myristate 13-acetate (PMA) purchased from Calbiochem 
(Billerica, MA). All the antibodies used to label and differentiate various blood leukocytes are obtained from BD biosciences.

\subsubsection{Preparation of SWCNTs-DNA dispersions}

SWCNTs are mixed with aqueous ss-DNA (GT) $)_{30}$ sequence with FAM-Q fluorescent tag, solution (approximately $1 \mathrm{mg} / \mathrm{ml}$ concentration) in an eppendrof tube. The mixture is sonicated in a cup horn sonicator (Cole Parmer 750W CPX) for about 60 $90 \mathrm{~min}$. The sample is then centrifuged at $16,000 \mathrm{~g}$ for 60 minutes (Eppendorf centrifuge 5415D), and the supernatant containing individually separated SWCNTs in ss-DNA solution are collected and resuspended in either 1x PBS or cell culture medium. ss-DNA wrapped SWCNTs were imaged using fluorescence microscopy (Nikon TE Eclipse 2000).

\subsubsection{Blood Isolation}

Blood $(\sim 8 \mathrm{ml})$ is drawn from healthy volunteers by venipuncture in accordance with protocols approved by the University of Louisville Institutional Regulatory Board (IRB). The drawn blood samples are then collected in green top vacctuainers and heparin is used as anticoagulant. The blood samples are maintained on ice until use. All the blood samples are obtained from Dr.Ashok Raj in the School of Medicine at the University of Louisville.

\subsubsection{Isolation of the PBMCs from whole blood}

As previously described, monocytes mature into antigen presenting cells (APCs) such as dendritic cells or macrophages. CD 83 is successfully used as a selective marker for the identification of the cells belonging to dendritic cells lineage. ${ }^{94}$, 95 The differentiation of monocytes into $\mathrm{CD} 3^{+}$dendritic cell/ macrophage phenotype (referred 
to as macrophages from this point forward) is accomplished as described by Zhou et al. ${ }^{96}$ To evaluate the immune response of the blood cells to SWCNTs, the isolation of monocytes from whole blood needs to be accomplished. Blood cells with round nuclei such as monocytes and lymphocytes including $T$ cells, B cells and natural killer (NK) are collectively referred to as peripheral blood mononuclear cells (PBMCs). They are isolated from human peripheral blood through a process called density gradient centrifugation which is based on differential migration of the different cell types during centrifugation due the presence of a density gradient. Ficoll-paque, a hydrophilic polysaccharide is used in density gradient media. Briefly, blood is carefully layered on top of the ficoll-paque solution and the sample is centrifuged. Following the centrifugation, the blood sample is separated into different layers as shown (Figure 3.10). The bottom layer contains ficoll-aggregated polymorphonuclear cells such as neutrophils and RBCs. The diffuse layer immediately above the bottom layer consists of mostly granulocytes and unbound ficoll solution. The PBMCs including the lymphocytes and monocytes are located the interface between the ficoll-granulocytes layer and the upper most plasma-platelet layer. The PBMCs are then carefully removed from the interface and washed with PBS or cell culture medium to remove any residual ficoll solution. 


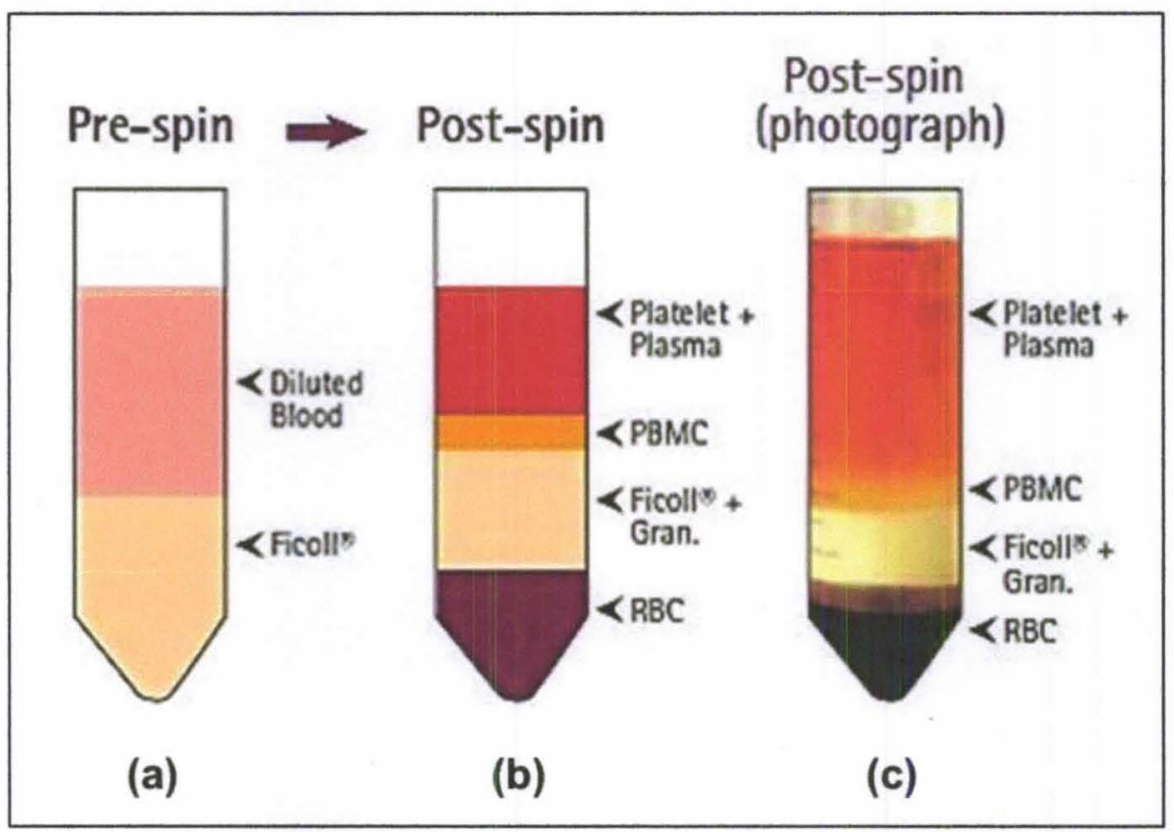

Figure 3.10 Isolation of PBMCs from whole blood. Schematic showing (a) layering of blood over ficoll before centrifugation, (b) the different layers after the density gradient centrifugation process, and (b) Representative photo of actual blood sample with isolated PBMCs. (Millipore)

The protocol followed in this dissertation for the isolation of PBMCs from blood is given below-

1. Prepare two aliquots of $2 \mathrm{~mL}$ of whole blood diluted $1: 1(\mathrm{v} / \mathrm{v})$ with $1 \mathrm{x}$ sterile PBS. Note: The dilution of the blood minimizes the aggregation of RBCs and PBMCs. Generally, the more diluted the blood sample, the better the purity of the PBMCs.

2. Carefully layer the diluted blood sample over ficoll-paque solution in 1:1 (v/v) in a two $15 \mathrm{ml}$ centrifuge tubes.

Note: The diluted blood sample is gently pipetted on top the ficoll by holding the tube at an angle to obtain a clean separation of the blood and ficoll layer. 
3. Centrifuge the sample for 20 minutes at $550 \mathrm{~g}$ at $4^{0} \mathrm{C}$ in a swinging bucket rotor without brake.

Note: Higher temperatures $\left(37^{\circ} \mathrm{C}\right)$ result in the aggregation of RBCs and thus reduce the yield.

4. Carefully remove the tubes from the centrifuge without disturbing the different layers.

5. Carefully extract the mononuclear cells layer at the interface to a new $15 \mathrm{ml}$ centrifuge tube containing $10 \mathrm{ml}$ of $1 \mathrm{x}$ sterile PBS.

6. Centrifuge the isolated mononuclear cells in $1 x$ PBS for 5 minutes at $1100 \mathrm{rpm}$ at $25^{\circ} \mathrm{C}$.

7. Discard the supernatant and resuspend the pellet containing the mononuclear cells in RPMI-1640 cell culture medium (ATCC 30-2001).

\subsubsection{Isolation of leukocytes from whole blood}

The leukocytes are isolated from the blood by lysis of the RBCs using tris buffer ammonium chloride $\left(\mathrm{NH}_{4} \mathrm{Cl}\right)$ lysis buffer. Briefly, $1 \mathrm{ml}$ of blood is mixed well with $15 \mathrm{ml}$ of $1 \mathrm{x}$ lysis buffer and incubated on a rotary shaker for 5 minutes at room temperature until the solution turns clear. The cells are then centrifuged for 5 minutes at $1000 \mathrm{rpm}$ at room temperature. The supernatant containing the lysed RBCs are discarded and the pellet is resuspended in $1 \% \mathrm{HAB}$ solution for subsequent experiments.

\subsubsection{Monocyte/macrophage maturation study}

The PBMCs isolated using the previously mentioned protocol are seeded and allowed to adhere to the surface of the tissue culture flasks. After approximately 8 hours the non-adherent cells (lymphocytes) are removed by pipetting. The remaining isolated 
monocytes are cultured with or without of SWCNTs. The differentiation of these monocytes into dendritic/macrophage phenotype cells is evaluated based on the expression of monocyte maturation markers (fluorescently labeled antibodies). The isolated monocytes are evaluated at four different time points- 0,2 and 4 day time points. At each time point, the cells are removed from the surface by trypsinization and resuspended in $200 \mu \mathrm{l}$ of $1 \mathrm{x}$ PBS. The cells are then split into two tubes containing $100 \mu \mathrm{l}$ of cell solution and mixed with $100 \mu \mathrm{l}$ of $1 \% \mathrm{HAB}$ solution and labeled with10 $\mu \mathrm{l}$ each of fluorescently labeled antibodies respectively. After 30 minutes of incubation at $4^{\circ} \mathrm{C}$, stained cells are washed with $500 \mu \mathrm{l}$ of $1 \% \mathrm{HAB}$ solution added to each of the two tubes. The cells are then centrifuged for 5 minutes at $1100 \mathrm{rpm}$ and resuspended in $250 \mu \mathrm{l}$ of $1 \%$ HAB solution. The cells are then transferred into two flow cytometer tubes and are analyzed for the phenotype (maturation) and activation markers using flow cytometry. Specifically, the expression of CD83 macrophage/dendritic cell marker is used to evaluate the maturation of the monocytes into macrophages. The internalization of SWCNTs:ss-DNA within macrophages is confirmed using fluorescently labeled ss-DNA by confocal microscopy (Nikon A1 Confocal System).

\subsubsection{Flow cytometry analysis}

The evaluation of cellular structure and functions and the interaction of molecules can be studied by fluorescent based methods like microscopy and flow cytometry. This section gives a brief description on the basics of flow cytometry. Flow cytometry is typically used to analyse various parameters of cells in a heterogeneous population and is widely used in immunophenotyping, cell counting, fluorescence expression analysis and also cell sorting applications. ${ }^{97-100}$ This analysis is performed by flowing large number 
(approximately thousands) of cells per sec through a laser beam. The properties of the cells such as their size, complexity, granularity, phenotype as well as their health is evaluated by gathering and analyzing the light emerging from each individual cells.

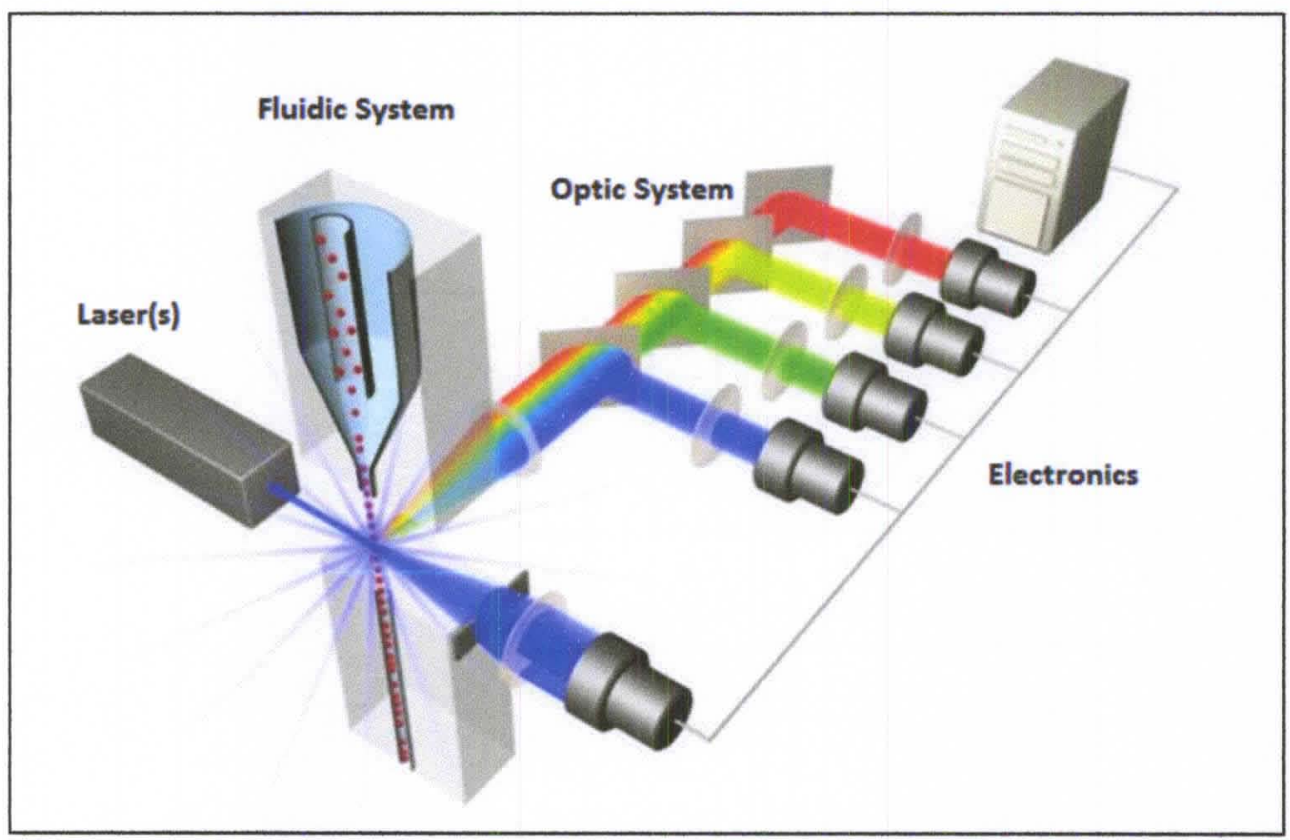

Figure 3.11 Schematic of flow cytometer showing the major four components - fluidic system, laser(s), optical system and the electronics for data analysis. (BD biosciences)

A typical flow cytometer has four major components which are briefly discussed below

\section{$\underline{\text { Fluidic system }}$}

A flow chamber or flow cell transports the cells through a laser to detect scattering and fluorescence emerging from the cells. The flow cell consists of a narrow channel through which the cells are injected under pressure surrounded by a sheath fluid. 
The fast flowing sheath fluid imparts huge drag in the flow cell which causes the cells to flow as a single stream. This effect is also called as hydrodynamic focussing.

\section{$\underline{\text { Laser system }}$}

When the laser is incident on the flowing cells, light is refracted and scattered in all directions. The amount of light refraction or scattering is dependent on the physical properties of the cellular population such as the size and the shape as well as the internal complexity such as granularity, membrane, nucleus material.

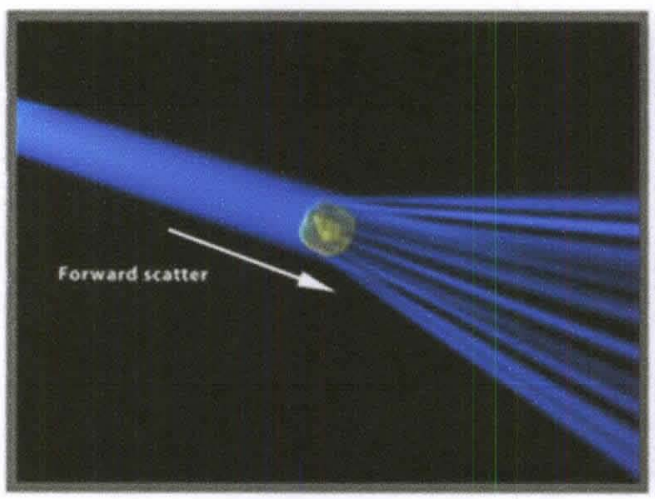

(a)

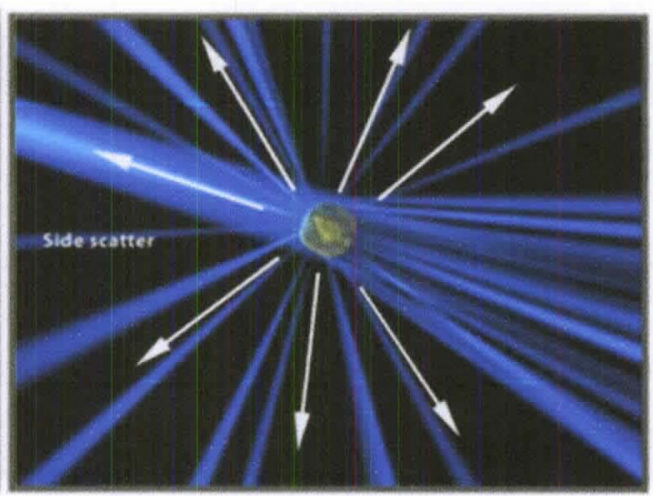

(b)

Figure 3.12 Schematic of light scattering and refraction of a cell flowing through the flow cell. (a) forward scatter, and (b) side scatter from individual cell (BD biosciences).

The scattering of the light is analyzed at two angles- forward scatter (FSC) and side scatter (SSC). The FSC is the light scattered in the direction of the laser excitation on the flowing cells population (Figure 3.12a). Smaller cells produce small amount of forward scatter while larger cells produce large amount of forward scatter. Therefore, a histogram of the FSC is a measure of the surface area or the size distribution within a 
population of cells. In the SSC, the light scattered by the cells at larger angles (i.e. to the side) are collected by focussing using a lens system usually at a right angle to the laser path (Figure 3.12b). SSC provides a measure of the granularity and internal complexity in the flowing cell population. A two dimensional histogram of the FSC and SSC data of the cell population is plotted as a dot or scatter plot. A dot plot of a typical blood sample is shown in the Figure 3.13 with each dot representing a cell in the population. For a typical blood sample, the smaller lymphocytes appear to the lower left on the $\mathrm{x}$ axis (R1- red) while the larger monocytes (R2- green) and granulocytes (R3- blue) are distinguished based on SSC data on the y axis.

\section{Optical system}

The laser system of a typical flow cytometer consists of an argon ion laser $(\sim 488$ $\mathrm{nm}$ emission) and a red diode laser ( $\sim 635 \mathrm{~nm}$ emission) used to excite the fluorescent antibodies used to label cells. The fluorescent antibodies can bind to specific molecules on the cell surface or inside the cell. The antibodies are selected in such a way that their peak emissions are not too close to each other during laser excitation. The most widely used antibodies are fluorescein isothiocyanate (FITC), peridin chlorophyll-a (PerCP), phycoerythrin (PE), and allophycocyanin (APC) conjugated antibodies. The $488 \mathrm{~nm}$ and the $635 \mathrm{~nm}$ laser excitation beams are combined and focussed on the cells using a focussing lens. The FSC data is collected using the FSC diode while the SSC data along with the fluorescence signals are collected using a collection lens at right angle to the laser beam. A series of optical filters and dichroic mirrors are used to spectrally split the

fluorescence signal which is collected by a photo multiplier tubes (PMTs). Different subpopulations of the cells from the total cells can be separated out by simultaneous 
labelling with different fluorescent antibodies. Typically, along with the FSC and SSC data, the fluorescence signal from a given subpopulation is used to determine the cell types as well as their percentages in the total cell sample.

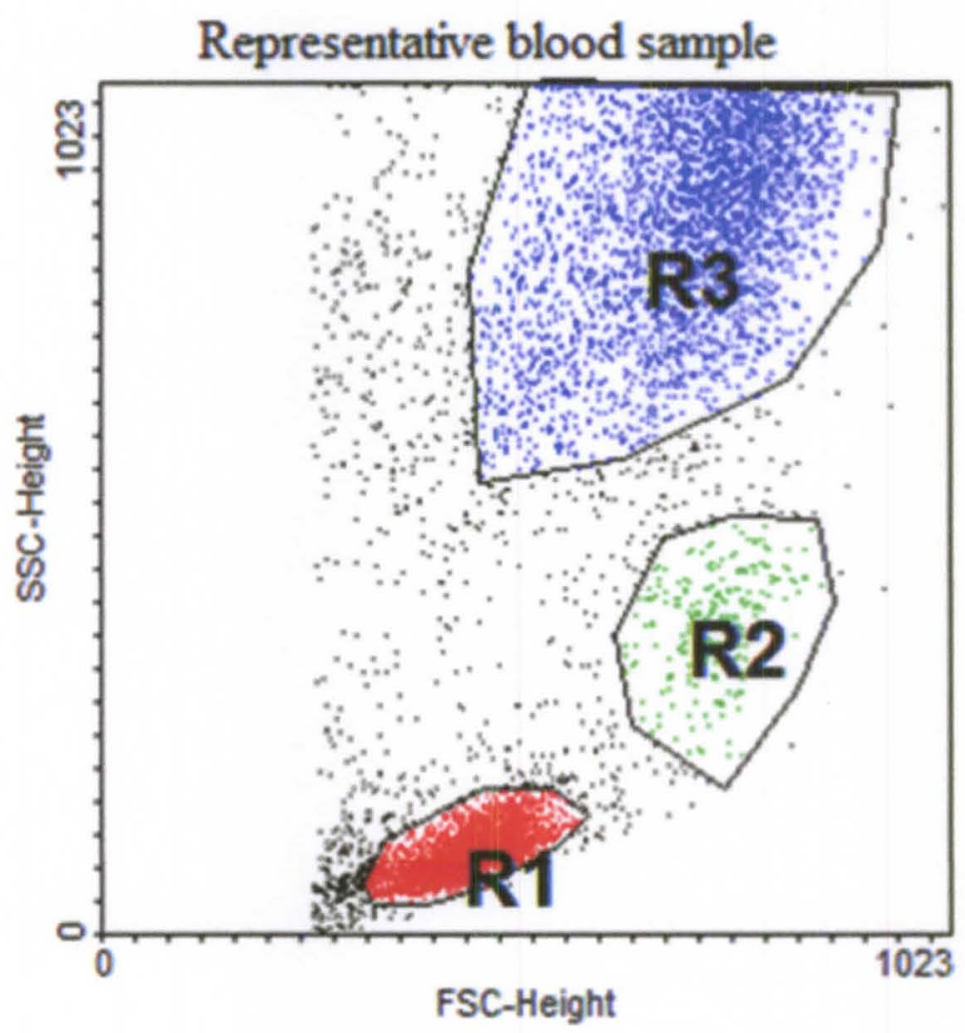

Figure 3.13 Representative dot plot from a typical blood sample showing three distinct cell populations depicted as three regions. R1 (red) represent the lymphocytes, R2 (green) represents the monocytes and R3 (blue) represents granulocytes.

\section{Electronics}

The electronics in the flow cytometer convert the light signals into voltage pulses using photodetectors such as photo diodes or photomultiplier tubes (PMTs).The voltage pulses are analyzed and quantified by signal processors to determine parameters like pulse width, height and area. The different subpopulations in the sample are isolated into 
subsets by creating graphical or numerical boundaries also known as gates. Softwares such as Cell Quest Pro or WinMDI are used to analyze and plot the data either as 2D-dot plots or histograms. A detailed schematic of the optical system layout for a BD FACS Calibur $^{\mathrm{TM}}$ flow cytometer system is shown in the Figure 3.14.

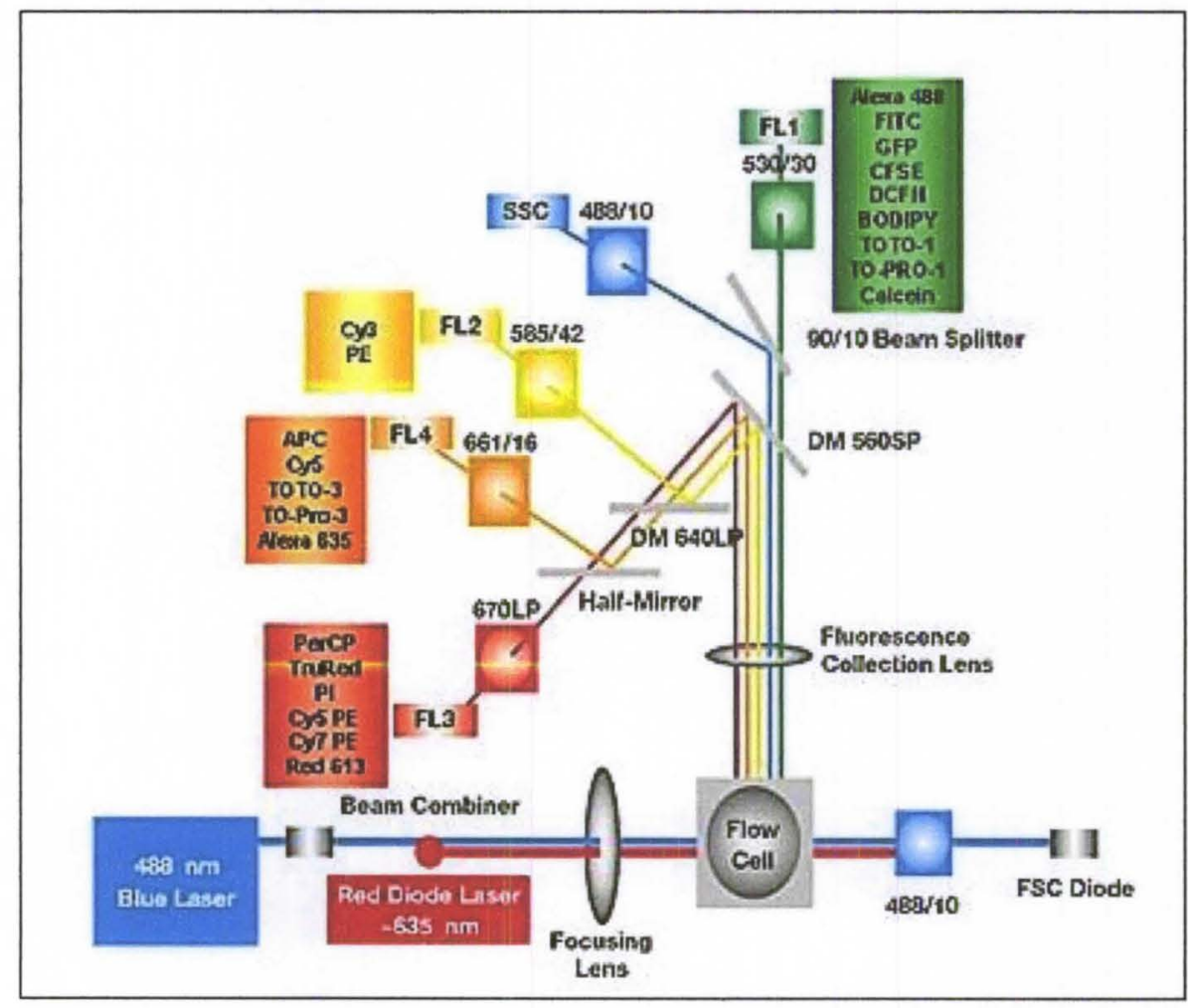

Figure 3.14 Schematic of BD FACS calibur flow cytometer system showing the optical system layout. (BD biosciences)

\subsection{EXPERIMENTAL SECTION}

In this dissertation study, the immune response of the blood leuckotyes to SWCNTs is evaluated by studying the interactions of the different subpopulations of the leukocytes to SWCNT using flow cytometry. The two different interactions recreated in 
this dissertation - direct interaction and an indirect interaction of the SWCNTs with blood will be discussed in the following sections.

\subsubsection{Direct interaction of SWCNTs with blood}

In the direct interaction study, the response of leukocytes in the blood when they are in direct contact with SWCNTs is evaluated. SWCNTs suspended in ss-DNA are added directly to $1 \mathrm{ml}$ whole blood and incubated for 2 hours at $37^{\circ} \mathrm{C}$ in $5 \% \mathrm{CO}_{2}$ incubator. The positive control for this study is blood mixed with PMA at a concentration of $\sim 100 \mathrm{ng} / \mathrm{ml}$ incubated for 2 hours at $37^{\circ} \mathrm{C}$. PMA, widely known potent tumor promoter is used to activate the leukocytes in the blood. ${ }^{101}$ Whole blood alone without SWCNTs or PMA is used as negative control. After 2 hours, blood leukocytes are isolated using $\mathrm{NH}_{4} \mathrm{Cl}$ lysis procedure and their response to SWCNTs is evaluated relative to negative controls (whole without SWCNTs) and positive controls (PMA) using flow cytometry analysis. Briefly, following the $\mathrm{NH}_{4} \mathrm{Cl}$ lysis, the cells are resuspended in $1 \% \mathrm{HAB}$ solution and split into three tubes (lymphocytes, monocytes and granulocytes) each with $100 \mu \mathrm{l}$ of cell solution. The cells solution is mixed with $100 \mu \mathrm{l}$ of $1 \% \mathrm{HAB}$ solution and labeled with $10 \mu \mathrm{l}$ each of fluorescently labeled antibodies as described previously. After 30 minutes of incubation at $4^{\circ} \mathrm{C}$, the cells are washed and resuspended in $250 \mu \mathrm{l}$ with $1 \%$ HAB solution for flow cytometry analysis. Cells are visualized using PerCP, FITC, PE and APC conjugated antibodies against leukocyte phenotype and activation cell-surface markers. The phenotype and activation markers are selected based on previous studies performed with analysis of leukocytes in response to bacterial stimulation ${ }^{102}$, burn and trauma injuries ${ }^{103}$ and sickle cell disease ${ }^{104}$. The list of the fluorescently labelled 
phenotype and activation markers used for all the different leukocyte subpopulations is given in the Figure 3.15 .

\section{Phenotype Activation}

\begin{tabular}{|c|c|c|c|c|}
\hline Cell type & FITC/FL1 & PE/FL2 & APC/FL4 & PerCP/FL3 \\
\hline Lymphocytes & CD3 & CD1a & CD4 & CD69 \\
\hline Monocytes & HLA/DR & CD11b & CD18 & CD14 \\
\hline Granulocytes & CD66b & CD11b & CD18 & CD69 \\
\hline
\end{tabular}

Figure 3.15 Phenotype (blue) and activation (grey) markers used to label the different subpopulation of blood leukocytes.

Lymphocytes are discriminated using FSC and SSC plots and are phenotyped into three distinct subpopulations using $\mathrm{CD} 3 / \mathrm{CD} 4$ markers. The $\mathrm{CD}^{+} \mathrm{CD}^{+}$represent the $\mathrm{T}$ helper1 lymphocytes $\left(\mathrm{T}_{\mathrm{h}} 1\right), \mathrm{CD}^{+} \mathrm{CD}^{-}$represent the $\mathrm{T}$ helper 2 lymphocytes $\left(\mathrm{T}_{\mathrm{h}} 2\right)$ and $\mathrm{CD}^{-} \mathrm{CD}^{-}$lymphocytes includes the B lymphocytes and Natural Killer (NK) cells. Monocytes and Granulocytes are discriminated using FSC and SSC and are phenotyped using CD14 and CD66b markers respectively. The expression of the different activation markers used for lymphocytes CD69 and CD1a; integrins CD11b, CD18 and human leukocyte antigen - DR (HLA-DR) or major histocompatibility complex II (MHC II) for monocytes; integrins CD11b, CD18 and CD69 for granulocytes is evaluated using flow 
cytometry. Flow cytometry data files are analyzed using WinMDI software (Scripps Institute). Each data file is manually gated and analyzed using different combinations of phenotype and activation markers. Histograms representing the mean fluorescence intensity (arbitrary units) for the specific activation markers for all the different subpopulations relative to their controls are plotted. All the data is represented as the percentage of cells phenotyped using certain phenotype markers (+/-) expressing high levels of the activation marker. The sample size for all the experiments is $n=4$ and data is represented as means of percentage of cells \pm SEM (standard error of mean).Statistical data analysis of the activation of the cells is scored using a rank sum test (Mann Whitney $\mathrm{U}$ test) with a $\mathrm{p}$ value less than 0.05 .

\subsubsection{Indirect interaction of SWCNTs with Blood}

The second study focuses on the interaction of blood cells with APCs containing SWCNTs. This study attempts to mimic the mechanism of presentation of foreign antigens to lymphocyte populations by macrophages and dendritic cells. The monocytes isolated from the whole blood are cultured with and without SWCNTs as mentioned previously. After 4 days, the cells are trypsinized and resuspended in cell culture medium. The cells are then mixed with fresh samples of $1 \mathrm{ml}$ whole blood respectively and kept on a gentle shaker platform for 30 minutes to extensively mix the blood with the macrophages. After mixing, the blood with the cells is incubated for 6 hours at $37^{\circ} \mathrm{C}$ in $5 \% \mathrm{CO}_{2}$ incubator. After 6 hours, the leukocytes from the blood are isolated using $\mathrm{NH}_{4} \mathrm{Cl}$ lysis, stained with the activation markers. The phenotype and activation markers used in this study are the ones used in the direct interaction study. The controls used in this study are whole blood without macrophages and whole blood with macrophages without 
internalized SWCNTs. The expression of the activation and phenotype markers are analyzed using flow cytometer as described in the previous section. All fluorescence expressions are normalized using controls and are represented as mean fluorescence intensity (MFI) in arbitrary units. The sample size for all the experiments is $n=4$ and data is represented as means of percentage of cells \pm SEM (standard error of mean). Statistical data analysis of the activation of the cells is scored using a rank sum test (Mann Whitney $U$ test) with a $p$ value less than 0.05 . All the data are plotted as histograms in the same way as in the direct interaction study.

The next chapter deals with the results obtained from the direct and indirect interaction experiments discussed above. The maturation of monocytes into macrophages and results from the flow cytometry data for all the different subpopulations of the blood leukocytes will be shown. 


\section{CHAPTER IV- RESULTS AND DISCUSSION}

\subsection{RESULTS}

This chapter deals with the results obtained from the monocyte/macrophage maturation study, SWCNTs: ss-DNA cell internalization study and direct and indirect interaction of SWCNTs with blood leukocytes.

\subsubsection{Monocyte Isolation and Maturation into APC Phenotype}

Monocytes are isolated from whole blood are cultured in tissue culture flasks for $0,2,4$ and 6 days in cell culture medium. The differentiation of monocytes into a macrophage/dendritic cell (APC) phenotype following culture is evaluated using the maturation marker CD83 (PE labelled) using flow cytometry. The percentage of cells with high expression of $\mathrm{CD} 83^{+}$is quantified and plotted for the different time points. It is observed from the analysis of maturation marker CD83 that on day 2 only $20 \%$ of cells showed high expression whereas on day 4 , more than $50 \%$ of cells expressed high levels of CD83 (Figure 4.1). Therefore, the monocytes which matured into macrophages after 4 days are used the APCs for the subsequent experiments. 


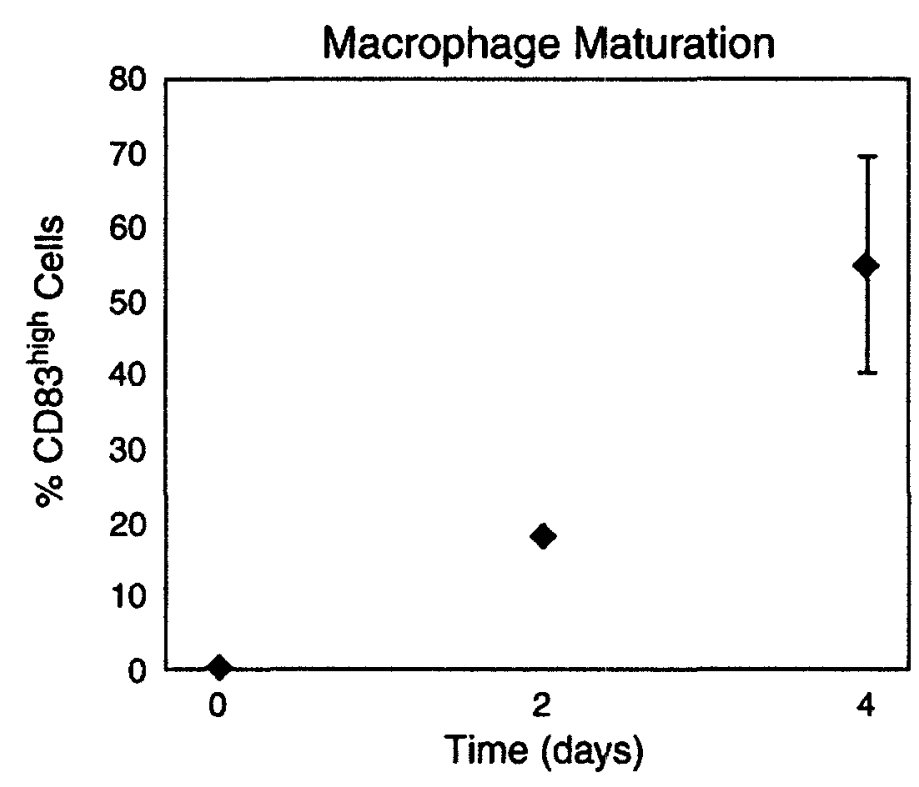

Figure 4.1 Monocytes/macrophage maturation study. SWCNTs internalized monocytes are tracked for using maturation marker (CD 83). The percentage of $\mathrm{CD} 83^{\text {high }}$ cells is shown for a sample size of $4 .^{105}$

\subsubsection{Internalization of SWCNTs:ss-DNA}

The monocytes isolated are cultured with SWCNTs functionalized with fluorescently labelled ss-DNA. After 6 hours, the cells are imaged under confocal microscopy to confirm the internalization of SWCNTs using the green fluorescence from the FAM-Q fluorescent dye attached to the ss-DNA. It is observed that the cells readily internalized the SWCNTs:ss-DNA via endocytosis like uptake mechanism as observed from the strong green fluorescence signal emerging from the cells. A representative confocal fluorescence image of single monocytes with and without SWCNTs is shown in the Figure 4.2. 

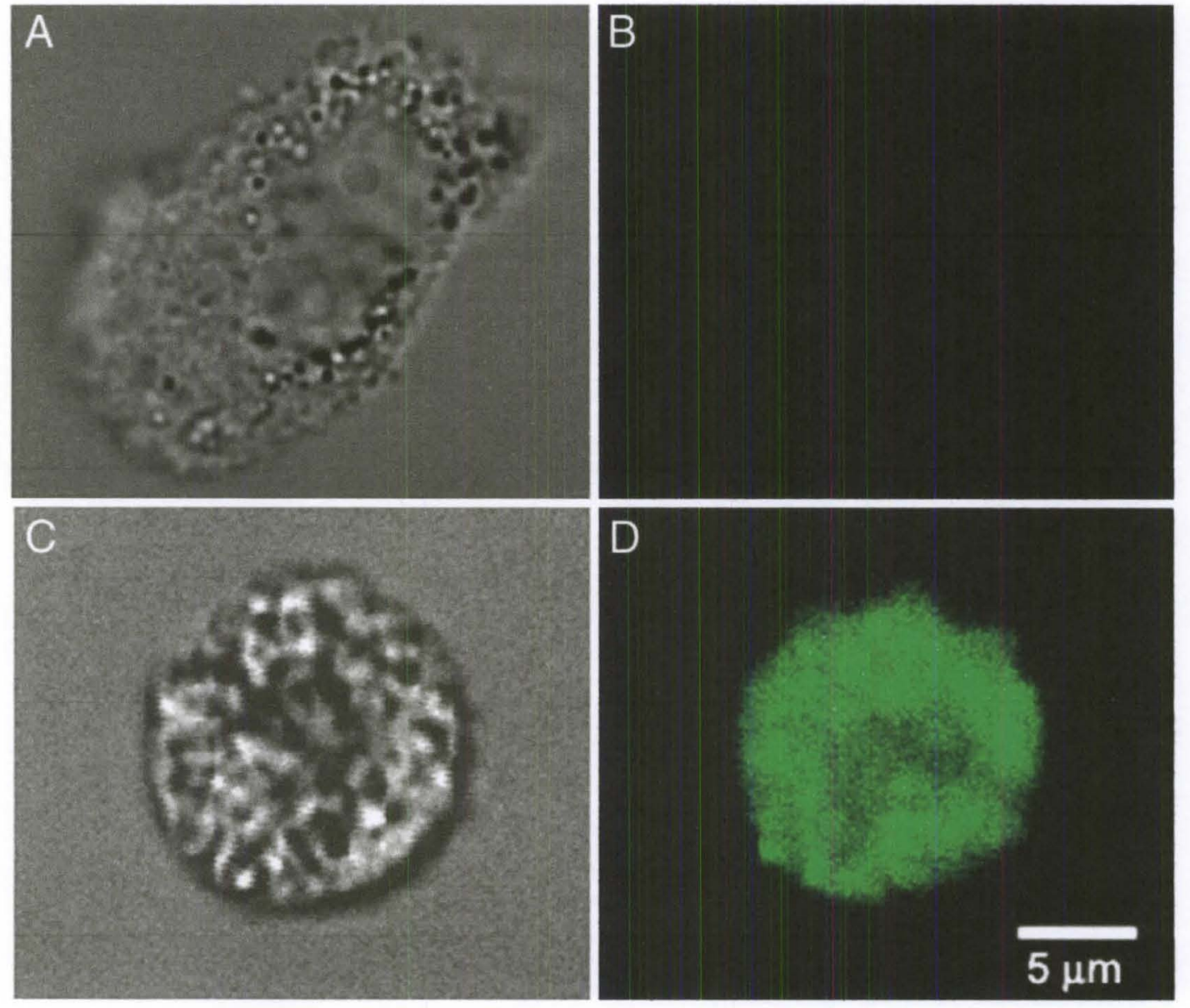

Figure 4.2 Confocal microscopy images of single monocytes with and without internalized SWCNTs:ss-DNA. Phase contrast and fluorescence images of cells (A), (B) without SWCNTs (control) and (C), (D) with SWCNTs functionalized with FAM-Q labelled ss-DNA. All the images are obtained at $60 \mathrm{x}$ magnification. ${ }^{105}$

\subsubsection{Direct interaction of SWCNTs with blood}

The response of blood leukocytes to ss-DNA functionalized SWCNTs is evaluated in comparison to negative controls (without SWCNTs) and positive controls (PMA) using flow cytometry analysis. The three different subpopulations of lymphocytes $\left(\mathrm{CD}^{+} \mathrm{CD}^{+}, \mathrm{CD}^{+} \mathrm{CD}^{-}\right.$and $\left.\mathrm{CD}^{-} \mathrm{CD}^{-}\right)$are evaluated for high levels of expression of 
activation markers CD69 and CD1a respectively. The expression of the activation markers relative to their respective controls is plotted as intensity histogram plots where the $y$-axis shows the number of events or the cell count and the $x$-axis $(\log )$ represents the mean fluorescence intensity of each of the activation markers (Figure 3.15). An increase in the fluorescence intensities of activation markers in the histograms represents increase in the activation of the different cell subpopulations. Flow cytometry analysis performed on a representative sample for the three different subpopulations of lymphocytes is shown in Figure 4.3. It is observed that the SWCNTs samples do not show increased activation in comparison to whole blood (negative control) for all the different subpopulations of the cells. However, analysis of the PMA stimulated (positive control) samples showed increased activation particularly for markers CD1a and CD69 on all lymphocyte subpopulations relative to whole blood (Figure 4.4). Further, monocytes and granulocytes are discriminated using FSC and SSC and are phenotyped using CD14 and CD66b respectively. The evaluation of the high levels of expression of the activation markers CD11b, CD18, HLA-DR on CD14+ monocytes and CD11b, CD18 and CD69 on $\mathrm{CD} 66 \mathrm{~b}+$ granulocytes is performed using the fluorescent intensity overlay histograms relative to respective controls.

It is observed that SWCNTs stimulated samples do not show any increased activation in comparison to whole blood (negative controls) both for the monocytes (Figure 4.5A) and granulocytes (Figure 4.6A). However, increased activation is seen for the PMA (positive control) stimulated sample particularly for markers CD11b, CD18 and CD69 on granulocytes (Figure 4.6B) and to a certain extent for CD11b, CD18 on monocytes (Figure 4.5B). Furthermore, the percentage of cells expressing high levels of 
the activation markers for all the different subpopulations of blood leukocytes are quantified from the flow cytometry data (Table 1). Cell activation is scored by performing statistical analysis of the flow cytometry data using a rank sum test with a $p$ value less than 0.05 and a sample size of 4 .

Statistical analysis of the data in Table 1 showed that when the unstimulated (whole blood) and ss:DNA functionalized SWCNTs stimulated samples are compared, there is no statistically significant difference in the expression of any activation marker for any of the leukocyte subpopulations. However when the unstimulated samples are compared to samples stimulated with PMA, the expression of several activation markers particularly $\mathrm{CD} 69$ on $\mathrm{CD}^{+} \mathrm{CD}^{+}$and $\mathrm{CD}^{+} \mathrm{CD} 4^{-}$lymphocytes, HLA-DR on monocytes and $\mathrm{CD} 11 \mathrm{~b}$ and $\mathrm{CD} 18$ on granulocytes all showed statistically significant changes. 


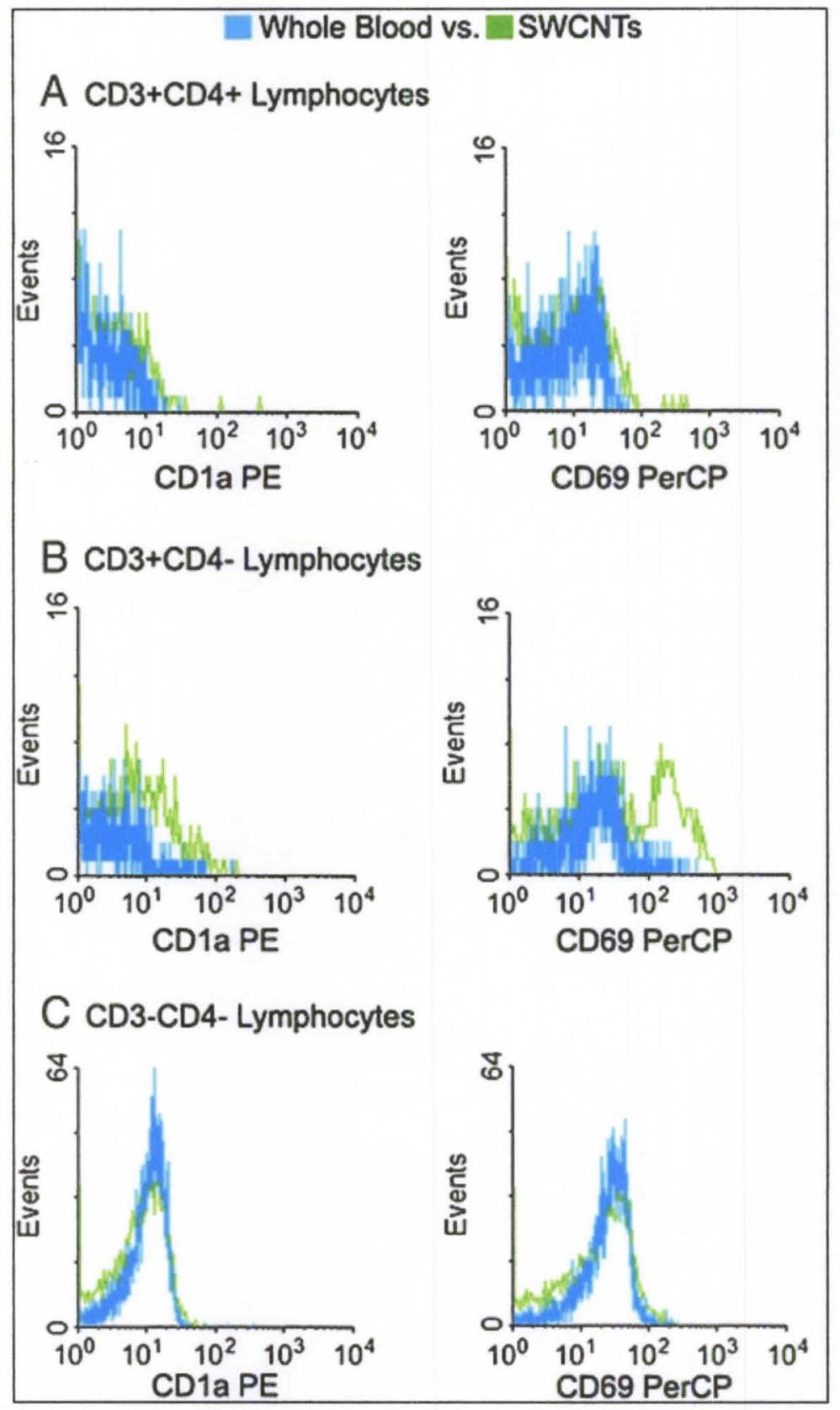

Figure 4.3 Direct interaction of SWCNTs with lymphocytes in the blood. Representative sample comparing expressions of CD1a and CD69 on (A) CD3+CD4+ lymphocytes, (B) CD3+CD4- lymphocytes and (C) CD3-CD4- lymphocytes in whole blood (negative control) vs. SWCNT-stimulated cells. All histograms represent mean fluorescence intensity (arbitrary units) for the specific activation marker. ${ }^{105}$ 


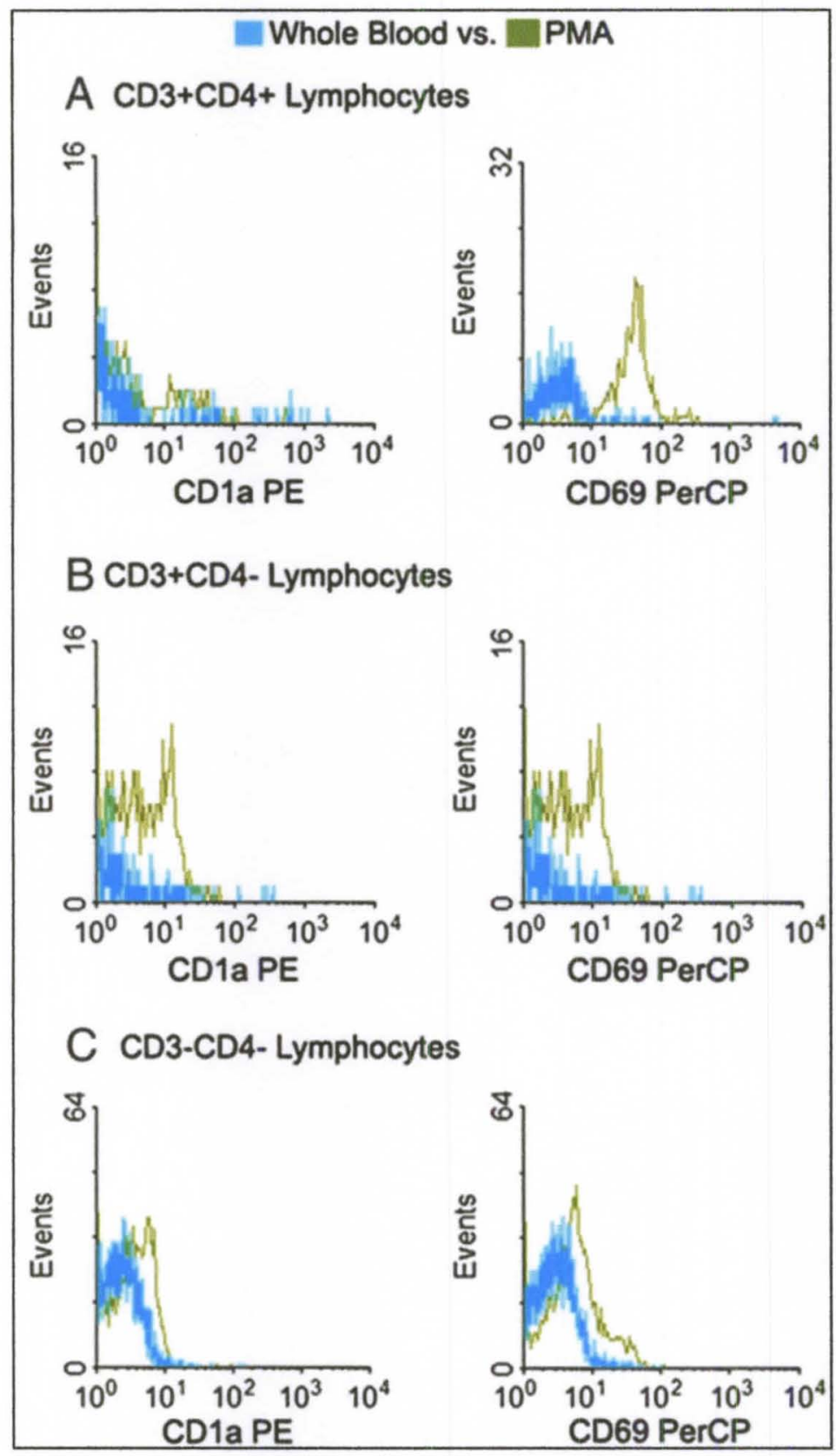

Figure 4.4 Direct interaction experiment lymphocytes controls. Representative sample comparing expressions of CD1a and CD69 on (A) CD3+CD4+ lymphocytes, (B) CD3+CD4- lymphocytes and (C) CD3-CD4- lymphocytes in whole blood (negative control) vs. PMA (positive control) stimulated cells. All histograms represent mean fluorescence intensity (arbitrary units) for the specific activation marker. ${ }^{105}$ 


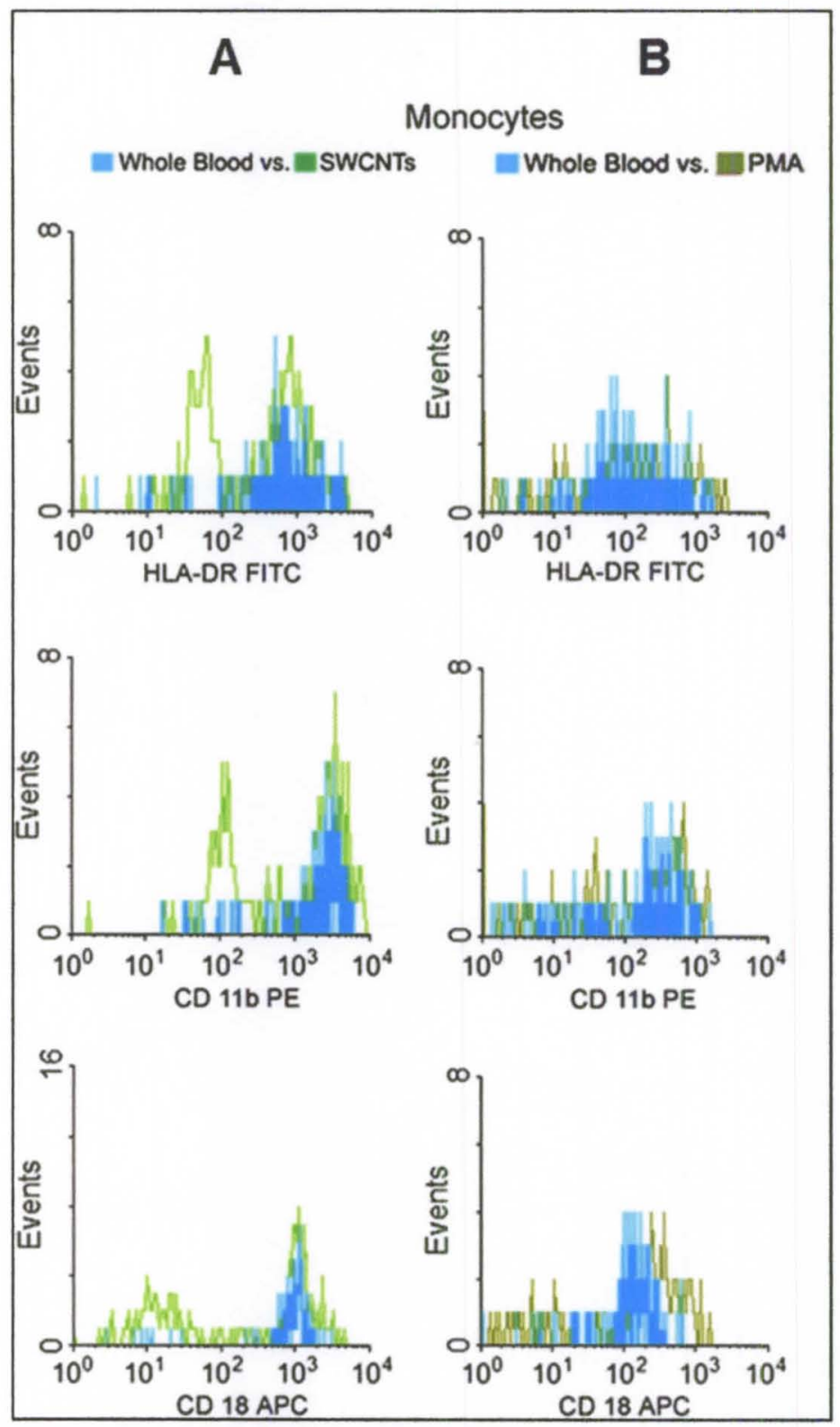

Figure 4.5 Direct interaction of SWCNTs with monocytes in the blood. Representative sample comparing expressions of CD11b, HLA-DR and CD18 markers on CD14+ monocytes in (A) whole blood (negative control) vs. SWCNTs-stimulated and (B) whole blood (negative control) vs. PMA-stimulated (positive control) blood samples. All histograms represent mean fluorescence intensity (arbitrary units) for the specific activation marker. ${ }^{105}$ 


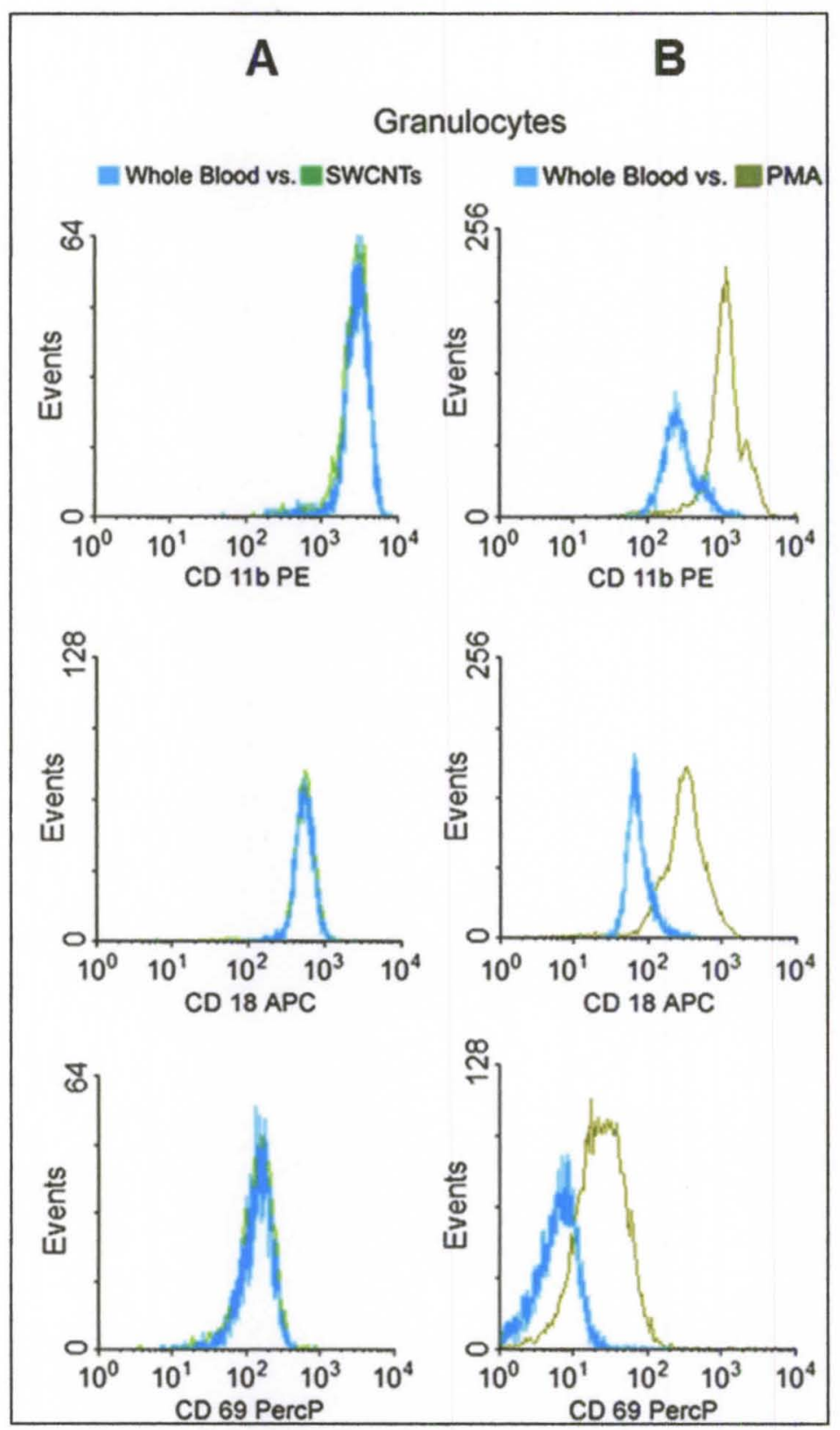

Figure 4.6 Direct interaction of SWCNTs with granulocytes in the blood. Representative sample comparing expressions of $\mathrm{CD} 11 \mathrm{~b}, \mathrm{CD} 18$ and $\mathrm{CD} 69$ markers on CD66b+ granulocytes in (A) whole blood (negative control) vs. SWCNTs-stimulated and (B) whole blood (negative control) vs. PMA-stimulated (positive control) blood samples. All histograms represent mean fluorescence intensity (arbitrary units) for the specific activation marker. $^{105}$ 


\begin{tabular}{|c|c|c|c|}
\hline Cell type & Blood & SWCNTs & PMA \\
\hline 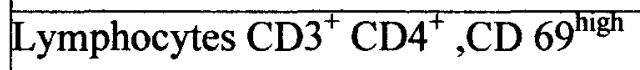 & $1.10 \pm 0.21$ & $1.26 \pm 0.66$ & $67.93 \pm 14.18$ \\
\hline 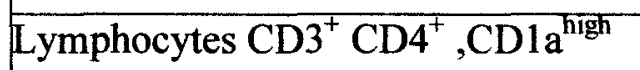 & $0.92 \pm 0.23$ & $3.37 \pm 2.67$ & $4.90 \pm 2.31$ \\
\hline Lymphocytes $\mathrm{CD}^{+}{ }^{+} \mathrm{CD} 4^{-}, \mathrm{CD} 69^{\text {high }}$ & $5.58 \pm 1.47$ & $9.64 \pm 7.24$ & $72.47 \pm 4.10$ \\
\hline${\text { Lymphocytes } \mathrm{CD}^{+}{ }^{+} \mathrm{CD}^{-}, \mathrm{CD} 1 \mathrm{a}^{\text {high }}}$ & $7.16 \pm 3.09$ & $9.71 \pm 7.62$ & $18.61 \pm 5.17$ \\
\hline Lymphocytes CD3- CD4- $4^{-}$CD $69^{\text {high }}$ & $21.24 \pm 21.16$ & $18.13 \pm 18.11$ & $10.37 \pm 4.25$ \\
\hline Lymphocytes CD $^{-} \mathrm{CD}^{-}, \mathrm{CD} 1 \mathrm{a}^{\text {high }}$ & $22.07 \pm 22.05$ & $19.52 \pm 19.46$ & $0.51 \pm 0.38$ \\
\hline Monocytes CD14 $^{+}$HLA/DR $^{\text {high }}$ & $72.37 \pm 4.17$ & $75.05 \pm 2.06$ & $30.37 \pm 8.91$ \\
\hline Monocytes CD14 $4^{+} \mathrm{CD} 11 \mathrm{~b}^{\text {high }}$ & $82.68 \pm 6.36$ & $79.04 \pm 5.39$ & $58.44 \pm 10.23$ \\
\hline Monocytes CD14 ${ }^{+} \mathrm{CD} 18^{\text {high }}$ & $82.08 \pm 5.93$ & $79.49 \pm 5.29$ & $57.95 \pm 9.63$ \\
\hline Granulocytes CD66 ${ }^{+} \mathrm{CD} 11 \mathrm{~b}^{\text {high }}$ & $39.01 \pm 20.16$ & $55.83 \pm 15.31$ & $91.83 \pm 6.29$ \\
\hline Granulocytes $\mathrm{CD} 6 \mathrm{~b}^{+} \mathrm{CD} 18^{\text {high }}$ & $33.94 \pm 21.96$ & $41.99 \pm 20.77$ & $89.94 \pm 6.73$ \\
\hline Granulocytes CD $66 \mathrm{~b}^{+} \mathrm{CD} 69^{\text {high }}$ & $24.77 \pm 24.75$ & $24.83 \pm 24.8$ & $15.49 \pm 15.10$ \\
\hline
\end{tabular}

Table 1. Direct response of blood leukocytes to SWCNTs and PMA. All data is expressed as $\%$ of cells \pm SEM of a particular phenotype (+/-) expressing high levels of an activation marker $(n=4) .{ }^{105}$

\subsubsection{Indirect interaction of SWCNTs with blood}

In the indirect interction study, the interaction of the blood leukocytes with APCs (macrophages) containing SWCNTs: ss-DNA is evaluated. The activation of the blood 
leukocytes due to the presentation of SWCNTs by the macrophages is investigated. Different subpopulations of the blood leukocytes are discriminated using FSC, SSC and the phenotype markers. Furthermore, the same panel of activation markers, CD69 and CD1a on all three lymphocyte populations; CD11b, CD18 and HLA-DR on monocytes; CD11b, CD18 and CD69 on granulocytes are evaluated. The expression of each of the activation markers for all the different subpopulations is plotted as fluoresce intensity histograms with respect to their controls (blood without macrophages and blood with macrophages without SWCNTs). Flow cytometry analysis performed on one representative sample is shown in Figure 4.7 for lymphocytes and Figure 4.8 (A, B) for monocytes and granulocytes respectively. It is observed that there is no increased activation in the cells with SWCNTs internalized macrophages in comparison to the controls for all the different lymphocytes, monocytes and granulocytes subpopulations. Cell activation is scored by performing statistical analysis of the flow cytometry data using a rank sum test with a p value less than 0.05 and a sample size of 4 (Table 2).

Statistical analysis of the data in Table 2 showed no statistically significant difference in the expression of activation markers on any of the leukocyte subpopulations stimulated with mature macrophages without SWCNTs or mature macrophages SWCNTs in comparison to the samples that are not mixed with macrophages. 


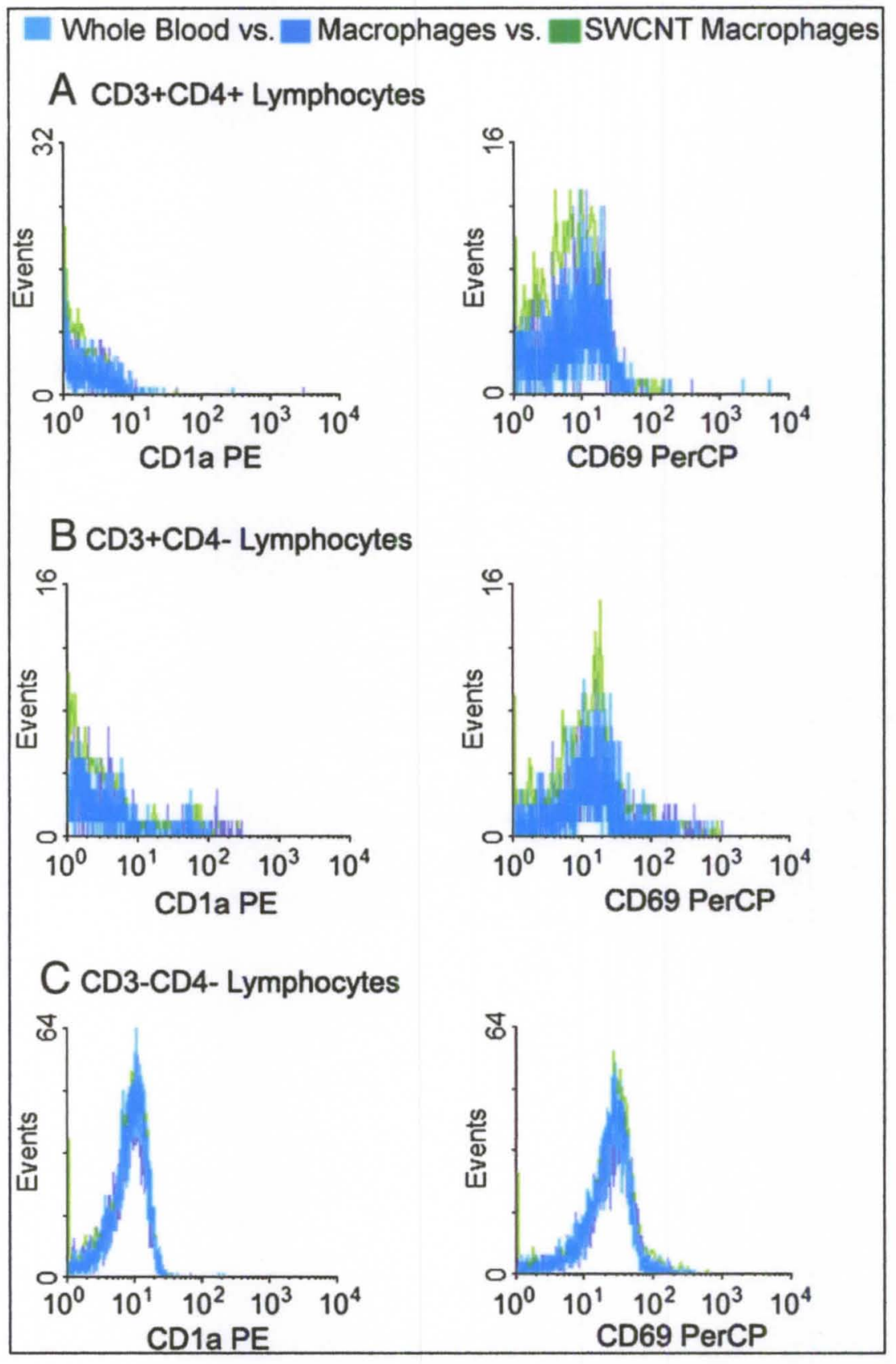

Figure 4.7 Indirect interaction of SWCNTs with lymphocytes in blood. Representative sample comparing expressions of CD1a and CD69 on (A) CD3+CD4+, (B) CD3+CD4and (C) CD3-CD4- lymphocytes in whole blood, stimulated with macrophage without SWCNTS and macrophages with internalized SWCNTS. All histograms represent mean fluorescence intensity (arbitrary units) for the specific activation marker. ${ }^{105}$ 


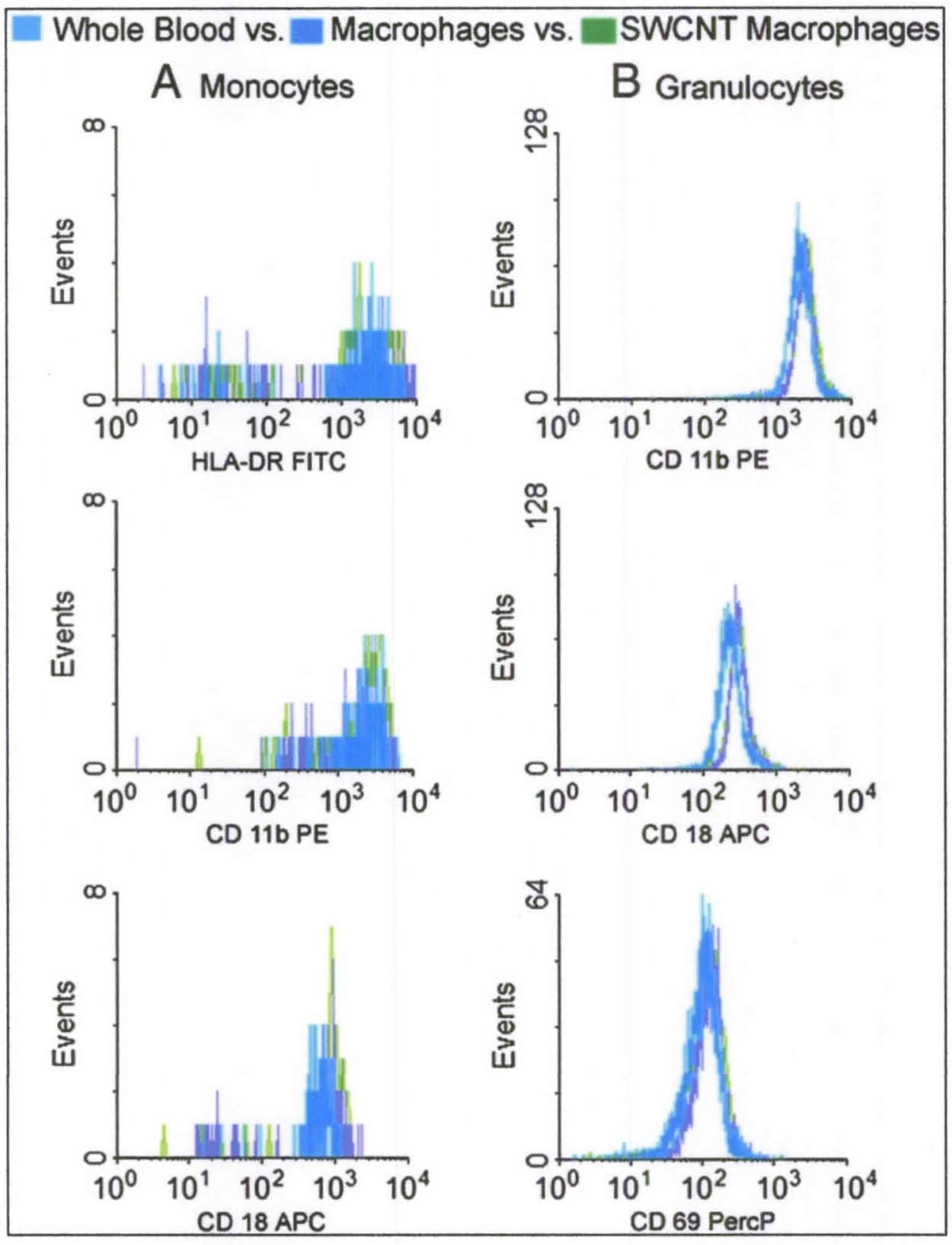

Figure 4.8 Indirect interaction of SWCNTs to monocytes and granulocytes in blood. Representative sample comparing expressions of (A) CD11b, HLA-DR and CD18 on CD14+ monocytes and (B) CD11b, CD69 and CD18 on CD66b+ granulocytes in whole blood, stimulated with macrophage without SWCNTS and macrophages with internalized SWCNTS respectively. All histograms represent mean fluorescence intensity (arbitrary units) for the specific activation marker. ${ }^{105}$ 


\begin{tabular}{|c|c|c|c|}
\hline Cell type & $\begin{array}{c}\text { Blood w/o } \\
\text { Macrophages }\end{array}$ & $\begin{array}{c}\text { Blood + } \\
\text { Macrophages } \\
\text { w/o SWCNTs }\end{array}$ & $\begin{array}{c}\text { Blood + } \\
\text { SWCNTs } \\
\text { Internalized } \\
\text { Macrophages }\end{array}$ \\
\hline 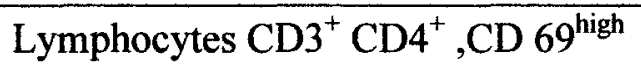 & $2.65 \pm 1.81$ & $2.75 \pm 1.51$ & $3.87 \pm 2.75$ \\
\hline 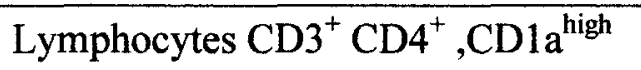 & $1.61 \pm 0.95$ & $1.01 \pm 0.35$ & $0.53 \pm 0.39$ \\
\hline 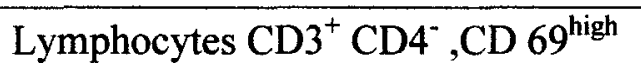 & $3.88 \pm 2.54$ & $5.32 \pm 3.37$ & $3.25 \pm 2.21$ \\
\hline 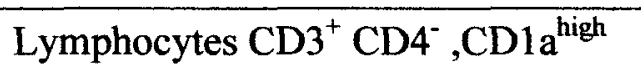 & $0.69 \pm 0.44$ & $1.91 \pm 1.14$ & $2.81 \pm 2.32$ \\
\hline Lymphocytes $\mathrm{CD}^{-} \mathrm{CD}^{-}, \mathrm{CD} 69^{\text {high }}$ & $1.48 \pm 1.10 \mathrm{~s}$ & $1.51 \pm 0.93$ & $1.02 \pm 0.59$ \\
\hline Lymphocytes $\mathrm{CD}^{-} \mathrm{CD} 4^{-}, \mathrm{CD} 1 \mathrm{a}^{\text {high }}$ & $0.315 \pm 0.26$ & $0.52 \pm 0.42$ & $2.10 \pm 2.04$ \\
\hline Monocytes CD14 ${ }^{+}$HLA/DR ${ }^{\text {high }}$ & $42.65 \pm 15.25$ & $34.02 \pm 12.78$ & $45.17 \pm 14.24$ \\
\hline Monocytes CD14 ${ }^{+} \mathrm{CD} 11 \mathrm{~b}^{\text {high }}$ & $54.16 \pm 17.1$ & $42.34 \pm 16.23$ & $49.51 \pm 15.4$ \\
\hline Monocytes $\mathrm{CD} 14^{+} \mathrm{CD} 18^{\text {high }}$ & $33.4 \pm 19.14$ & $24.64 \pm 14.1$ & $32.89 \pm 18.41$ \\
\hline Granulocytes $\mathrm{CD}^{2} 6 \mathrm{~b}^{+} \mathrm{CD} 11 \mathrm{~b}^{\text {high }}$ & $72.34 \pm 21.61$ & $80.77 \pm 17.1$ & $77.97 \pm 19.94$ \\
\hline Granulocytes $\mathrm{CD} 6 \mathrm{~b}^{+} \mathrm{CD} 18^{\text {high }}$ & $23.21 \pm 23.04$ & $24.1 \pm 26.63$ & $24.26 \pm 23.84$ \\
\hline Granulocytes $\mathrm{CD} 6 \mathrm{~b}^{+} \mathrm{CD} 69^{\text {high }}$ & $1.64 \pm 1.51$ & $6.83 \pm 6.69$ & $4.22 \pm 4.1$ \\
\hline
\end{tabular}

Table 2. Indirect response of blood leukocytes to SWCNTs in comparison to whole blood, stimulated with macrophage without SWCNTS and macrophages with internalized SWCNTS. All data is expressed as \% of cells \pm SEM of a particular phenotype (+/-) expressing high levels of an activation marker $(n=4) .{ }^{105}$ 


\subsection{DISCUSSION}

Carbon nanotubes are widely used for various applications in cell and molecular biology due to their unique physical and structural properties. ${ }^{5,29-31,33-35}$ Several studies have reported on the great potential of CNTs in transporting various biomolecules like antibodies, proteins and nucleic acids into the cytoplasm and potentially into the nucleus. ${ }^{60,} 62,70,71,106$ Several reports exists on the cellular level toxicity of CNTs in a variety of mammalian cells including leukocytes isolated from blood; however while majority of these studies reported minimal changes to cell structure and function ${ }^{85-87}$, some studies observed changes in cell signalling processes due to CNTs. ${ }^{107,}{ }^{108}$ Cell culture models provide limited information regarding the systemic response of the body due to the presence of CNTs.

In this dissertation, blood is chosen as an appropriate tissue culture model to thoroughly investigate the response of the SWCNTs in the human body. Blood is a living tissue and plays a major role in immune homeostasis. Therefore, the response of the body to SWCNTs can be evaluated by studying the changes in the inflammatory status of the cellular populations (specifically leukocytes) in the blood. Due to the limited stability of the blood cells in vitro( $\sim 6$ hours), the studies are limited to investigating the acute response of blood to SWCNTs. The acute immune response is investigated by extracting blood samples and studying the interactions of SWCNTs with blood leukocytes. Two possible types of interactions of SWCNTs with blood are recreated - direct interaction and indirect interaction. The direct interaction mimics the presence of SWCNTs in blood circulation while the indirect interaction recreates the presentation of SWCNTs to the blood leukocytes via APCs. The direct interaction study is analogous to the innate 
immune response while the indirect interaction study is analogous to the adaptive immune response which occurs in the human body in response to foreign antigens. The activation markers chosen for monocyte and granulocyte subpopulations are integrins and MHC II (HLA-DR) molecules that the cells express in response to any foreign antigens. As mentioned in chapter III, integrins help in adhesion of leukocytes to endothelium and promote transmigration into site of tissue inflammation, which is a defining event in the early activation of monocytes and granulocytes. The activation markers chosen for lymphocytes are cell surface molecules that are upregulated following their interaction with APCs like macrophages and dendritic cells. Therefore, by evaluating the expression of the integrins, MHC II and cell surface molecules, the immune response of all the different subpopulations of the blood leukocytes can be very well understood. Any activation of leukocytes due to SWCNTs is reflected as changes in the expression of these activation markers.

Results from the direct interaction of SWCNTs with blood showed that there is no increased expression of the activation markers relative to the negative control for the different lymphocyte, monocyte and granulocyte subpopulations. However, the tumor promoter, PMA stimulated samples (positive control) show distinct increase in the expression of most of the activation markers for the different subpopulations of the cells. These results are further corroborated using the statistical rank sum test which indicates no statistically significant difference in the expression of the activation markers on the SWCNTs samples versus the blood (negative control) samples. These results indicate minimal activation of blood leukocytes following their direct interaction with SWCNTs. Thus, the leukocytes do no initiate an immune response due to the presence of SWCNTs 
in the blood circulation. In the indirect interaction of SWCNTs with blood, the results obtained showed that there is no increase in the expression of any of the activation markers for all the different leukocyte subpopulations. The expression of the activation markers for the leukocytes samples stimulated with SWCNTs internalized macrophages is very similar to the samples stimulated with macrophages without SWCNTs and samples without any macrophages. The statistical test further confirmed that there is no statistically significant difference in the expression of the activation markers on all leukocyte subpopulations in all the three different samples. These results indicate minimal activation of blood leukocytes following their interaction with SWCNTs via APCs. Thus, the presentation of SWCNTs by the APCs to the blood leukocytes does not initiate an immune response from the leukocytes. Though the long-term effects are not observed, this thorough study provides a good starting point in trying to evaluate leukocyte activation in response to SWCNTs in the body. The ss-DNA used to functionalize SWCNTs serves two purposes. Firstly, it as a surfactant preventing aggregation in the suspension medium and secondly, it provides a highly flexible backbone for further functionalization with biomolecules targeting specific groups on the $5^{\prime}$ and $3^{\prime}$ ends of the strand. Further, the intracellular delivery of ss-DNA using SWCNTs is relevant to gene and drug delivery applications. The results demonstrate minimal acute immune response from blood leukocytes and support the pursuit of CNTs based approaches for delivery of genes and biomolecules to cells.

\subsection{SUMMARY}

This concludes the first part this dissertation in which the use of CNTs for biomedical applications is discussed. The toxicity of CNTs and their potential to elicit an 
immune response from blood leukocytes is evaluated. This is achieved by recreating, in vitro, two types of interactions that potentially occur in the body - (a) direct interaction: due to the presence of CNTs within the body and (b) indirect interaction: due to the presentation of CNTs via APCs to the blood leukocytes. Blood leukocytes are isolated and evaluated for the expression of early activation markers. The results indicate minimal leukocytes activation following both types of interactions. The potential of CNTs to transport of various biomolecules like genes and drugs across the cell membrane is also demonstrated by delivery ss-DNA. The results support the pursuit of CNTs based approaches for delivery of genes and biomolecules to cells.

The next part of this dissertation discusses semiconductor nanocrystals or quantum dots (QDs) for the intracellular labelling and tracking of subcellular biomolecules. 


\section{CHAPTER V- COLLOIDAL QUANTUM DOTS}

\subsection{INTRODUCTION}

Semiconductor nanocrystals or quantum dots (QDs) are inorganic semiconductor particles with diameters ranging from a few nanometers to hundreds of nanometers that possess size dependent electronic and optical properties ${ }^{109-112}$. At a given temperature, the size below which variation in the fundamental electrical and optical properties occurs when the energy level spacing exceeds the temperature is large in the case of semiconductors than in metals, insulators or molecular crystals. In metals, the fermi level lies in the center of a band and there is very small energy level spacing, and at few kT, the electrical and optical properties resemble that of continuum even in small sized nanocrystals ${ }^{113}$. However, in semiconductors, fermi level lies between two bands and the band edges dominate the low energy optical and electrical properties. (Figure 5.1) The size dependent properties of these nanocrystals are a result of two major factors. Firstly, the number of atoms on the surface is a large fraction of the total number. Secondly, the fundamental properties of these nanocrystals are determined by quantum confinement effect.

\subsection{QUANTUM CONFINEMENT}

In a semiconductor material, the superposition of wave functions and energy states of all the atoms result in the band structure. The minimum energy required to excite 
an electron into the vacant conduction band leaving behind a hole in the filled valence band is the band gap energy, $\mathrm{Eg}$.

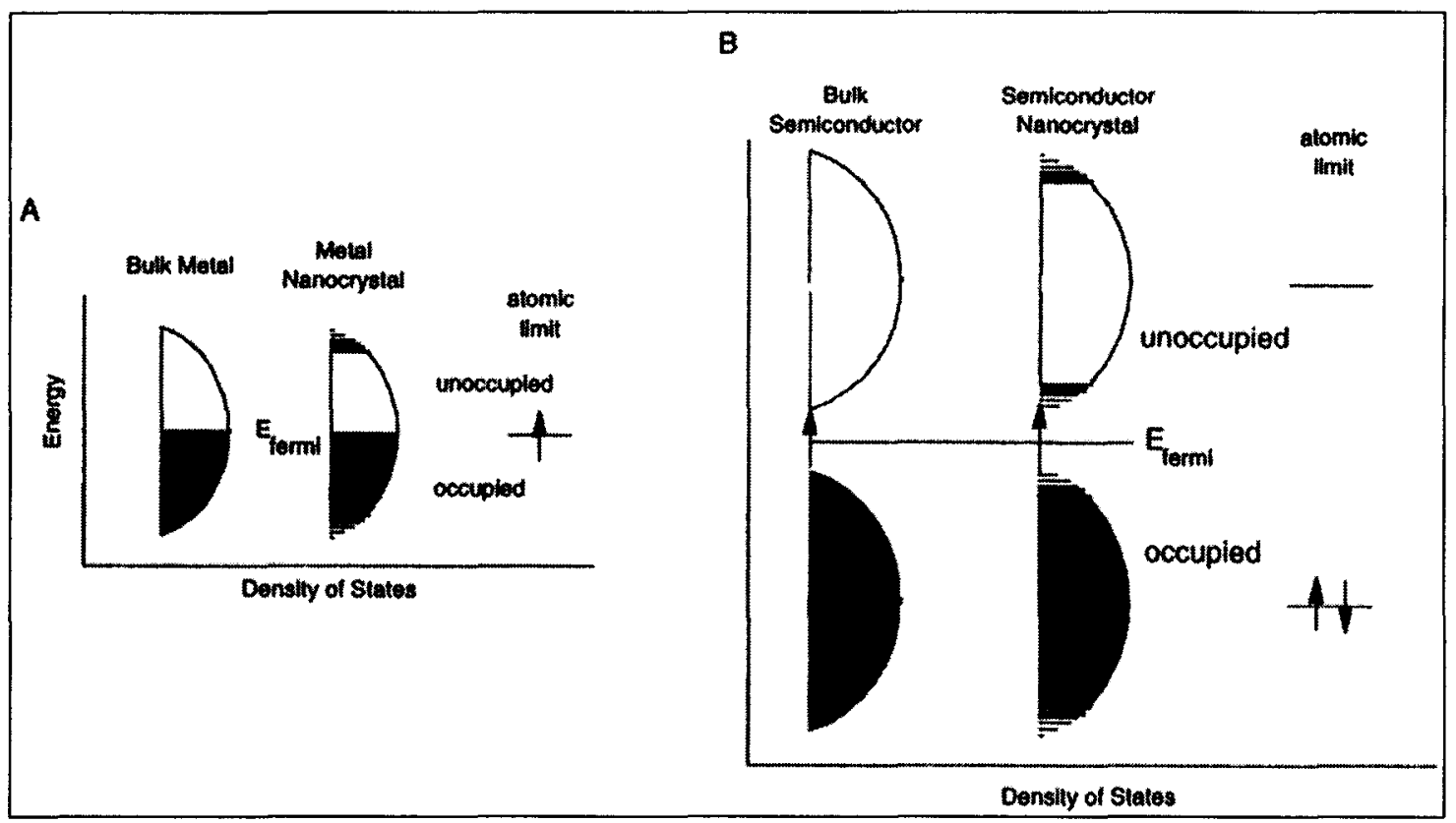

Figure 5.1 Density of states comparison of (A) metal and (B) semiconductor nanocrystals with respective bulk materials. The density of states is discrete in both metals and semiconductors near the band edges. In metal nanocrystals, as fermi level lies in the center of band, a few kT is greater than the energy level spacing and hence it resembles continuous states even for small sizes. However, in semiconductor nanocrystals, as the fermi level lies in between two bands, the energy level spacing is large even at large sizes and the energy gap increases in semiconductor nanocrystals of smaller size. ${ }^{110}$

Typically, the electronic transitions in the semiconductor material determine its optical properties. An exciton is created when the electron-hole pair is electrostatically bound. Radiative recombination can occur when the excited electron relaxes back to the valence band by emitting a photon. The size of the exciton varies from a few to few 
hundreds of nanometers and is defined by Bohr exciton radius $\left(a_{e x}\right)$. The radius of the exciton is defined by a physical constant called Bohr radius $\left(a_{B}\right)$, the approximate distance between the electron and proton in a hydrogen atom in its ground state. (Equation 5.1)

$$
a_{B}=\frac{4 \pi \varepsilon_{0} \hbar^{2}}{m_{e} e^{2}}
$$

Equation 5.1 Formula for Bohr radius $a_{B}$, where $\varepsilon_{0}$ is the permittivity of free space, $\hbar$ is the reduced Planck's constant, $m_{e}$ is electron rest mass, $\mathrm{e}$ is the charge.

The exciton Bohr radius $\left(a_{e x}\right)$ is obtained by scaling the Bohr radius $\left(a_{B}\right)$ with dielectric scaling factor. (Equation 5.2)

$$
a_{e x}=\frac{\kappa \hbar^{2}}{\mu e^{2}}
$$

Equation 5.2 Formula for exciton Bohr radius, $a_{e x}$, where $\mu$ is the exciton reduced mass, $\kappa$ is the dielectric constant of the semiconductor.

When the size of the semiconductor approaches the exciton Bohr radius or the de-Broglie wavelength, quantum confinement effects are observed. The de-Broglie wavelength $\lambda$ is given by the following equation

$$
\lambda=\frac{h}{p}=\frac{h}{\sqrt{3 k T m_{\text {eff }}}}
$$

Equation 5.3 The de-Broglie wavelength $\lambda$ depends on the effective mass $m_{\text {eff }}$ of the carrier (electron or hole) in the crystal not the free electron mass and also on the 
temperature $\mathrm{T}$. The effective mass in the crystal is much smaller than the free electron mass.

In the late 1950s and early 1960s, research has mainly focused on fabricating ultra-thin films of semimetals like bismuth for studying the size quantization effects. ${ }^{114}$ Lustkii et al. and Filatov et al. have observed the effect of the increase in the band gap with decreasing thickness of $\mathrm{Bi}$ and InSb films. ${ }^{115,}{ }^{116}$ However, most of the work during that time was theoretical due to the limited availability of technology to fabricate high quality interfaces. The possibility of observing size quantization effects are reduced due to rough or defective interfaces. In the late 1960 s, development of novel epitaxial deposition techniques such as molecular beam epitaxy and metal organic chemical vapor deposition led to the fabrication of multilayer periodic semiconductor heterostructures. Now, it is experimentally well established that the size and shape of a semiconductor can have profound effects on its electronic and optical properties. ${ }^{17,118}$

\subsection{ELECTRONIC STRUCTURE OF ODS}

In bulk semiconductor or 3D system, the exciton can move freely in all directions. If the material size is reduced in one dimension i.e., the exciton can move freely in only two dimensions, the resultant structure is referred to as quantum well (QW) or $2 \mathrm{D}$ well. In this case, the electrons behave like electrons in a potential well and have discrete energy levels due to quantum mechanical confinement. Quantum wells were formed by inserting very thin layers (approximately few lattice constants thick) of a lower bandgap semiconductor in a matrix with larger bandgap. One of the first quantum wells were fabricated in the $\mathrm{GaP}_{x} \mathrm{As}_{1-\mathrm{x}} / \mathrm{GaAs}$ material system in early 1970 s by Noble 
prize winner Alferov. ${ }^{119}$ By the end of 1980 s, the fundamental properties of these quantum wells were well understood and the research focused on fabricating structures with further reduced dimensionality. ${ }^{120}$ If the exciton is confined in two dimensions and is free to move in only one dimension, then it is termed as quantum wire (QWR) or 1D wire. In the case of quantum dot (QD), the exciton is restricted in all three dimensions and the confinement is more pronounced than the QW or the QWR case. They are referred to as $0 \mathrm{D}$ structures as the carriers are completely confined in all three dimensions which results in discrete energy levels as in the case of the electron in a hydrogen atom. A comparison of a 3D bulk material with a $2 \mathrm{D}$ quantum well, a $1 \mathrm{D}$ quantum wire and a quasi 0D QDs is shown (Figure 5.2).

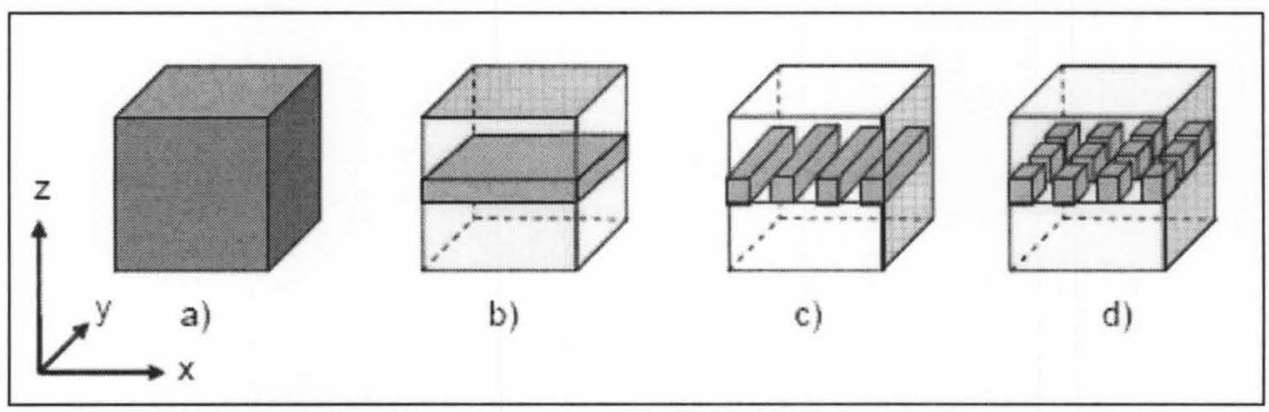

Figure 5.2 Schematic of (a) 3D or bulk, (b) 2D or quantum well, (c) 1D or quantum wire, and (d) $0 \mathrm{D}$ or quantum dot is shown. ${ }^{117,118}$

Band structures of materials are calculated using free electron or KroningPenney or tight-binding models and effective mass and pseudopotential methods. The number of allowed energy states in the band structure are defined by the density of states (DOS). The densities of states are strongly influenced by quantum confinement effects as shown in the Figure 5.3. In bulk or 3D system, the DOS is continuous as the carriers are 
free to move in all directions. In the case of quantum well or 2D system, the carriers are confined in one dimension and thus DOS resemble a step function like behavior.

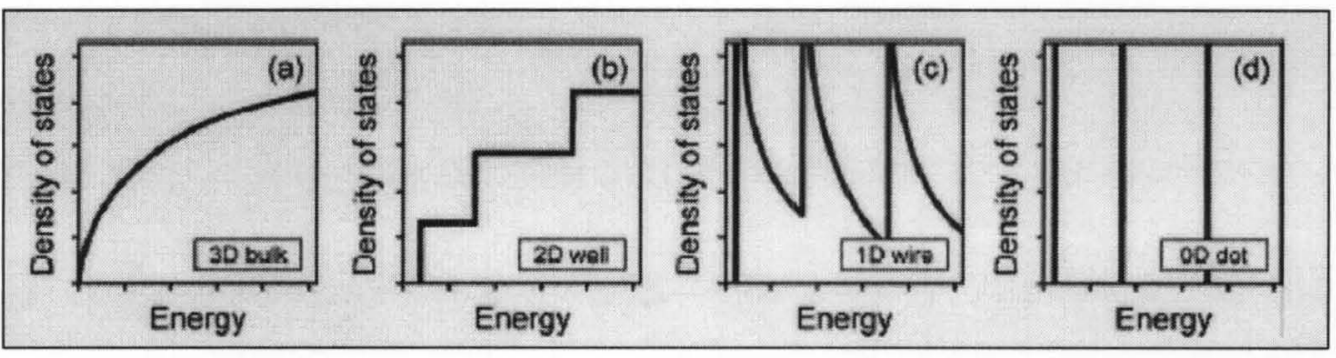

Figure 5.3 Densities of states of (a) 3D or bulk, (b) 2D or quantum well, (c) 1D or quantum wire, and (d) OD or quantum dot. The DOS is continuous in bulk, a step function in QW, has peculiarities in QWR and a delta function in QDs. ${ }^{117}$

In the case of quantum wire or $1 \mathrm{D}$ wire, the DOS has a peculiarity also referred to as Van-Hove singularities. In the case of QDs or 0D structures, the carrier localization occurs in all three dimensions which results in the breakdown of the classical band structure model. This strong quantum confinement results in very discrete energy states in the band structure of single QD similar to atomic energy states. This dissertation deals with such semiconductor nanocrystals or QDs.

In addition to the electronic properties, the quantum confinement affects the optical properties of these quantum confined structures. The optical absorption and emission properties of these structures are very different from the bulk materials. The exciton levels of the QD are given by solving the classical quantum mechanical problem of particle in a box. The minimum energy required to excite an electron from the valence band to the conduction band in the QD is given by 


$$
E=E_{g}+\frac{\hbar^{2} \pi^{2}}{2 r^{2}}\left(\frac{1}{m_{e}}+\frac{1}{m_{h}}\right)-\frac{1.8 e^{2}}{\varepsilon r}
$$

Equation 5.4 The lowest excited electronic state of a quantum dot is given by $\mathrm{E}$, where $E_{g}$ is bulk band gap energy, $\hbar$ is the reduced Planck's constant, $m_{e}$ and $m_{h}$ are the effective mass of the electron and hole, e is the electron charge, $\varepsilon$ is the dielectric constant and $r$ is the radius of the quantum dot.

As shown in the equation 5.4, the excited energy state of the QD is dependent on the band gap, the dielectric constant of the bulk material and on the size or radius of the QD. The second term in the equation 5.4 is quantum localization energy for electron and hole which increases with decreasing size as $1 / r^{2}$. The third term in the equation 5.4 represents the Coulomb energy which is a function of $1 / \mathrm{r}$. It can be seen that for large radius the Coulomb interaction is dominant than the quantization energies of the electrons and hole. In the case of small radius the Coulomb interaction is less important and the quantization energies dominate. Thus the optical properties not only depend on the composition of the material but also on the size and the shape of the QD. As the radius of the QD decreases, the spacing between the discrete energy levels increases resulting in blue shift in the optical emission spectrum (Figure 5.3). In other words, the smaller the dot, the more blue shifted is the fluorescence emission and the larger the dot, the more red shifted is its fluorescence emission.

\subsection{SYNTHESIS OF ODS}

During the early 1930's, Rocksby first realized QDs as nanometer sized semiconductor crystal inclusions in glass which are commercially available for decades 
as color filters. ${ }^{121}$ It was only in the early 1980's, QDs synthesis was described by Efros et al. and Ekimov et al. who experimentally confirmed the quantum confinement effects in such colored glass matrices by growing semiconductor nanocrystals and microcrystals in glass matrices. ${ }^{122,123}$ Since their work, techniques to synthesize of QDs received substantial attention and extensive research efforts. Early research to fabricate QDs focused on patterning quantum wells using high resolution lithography techniques. ${ }^{124-127}$

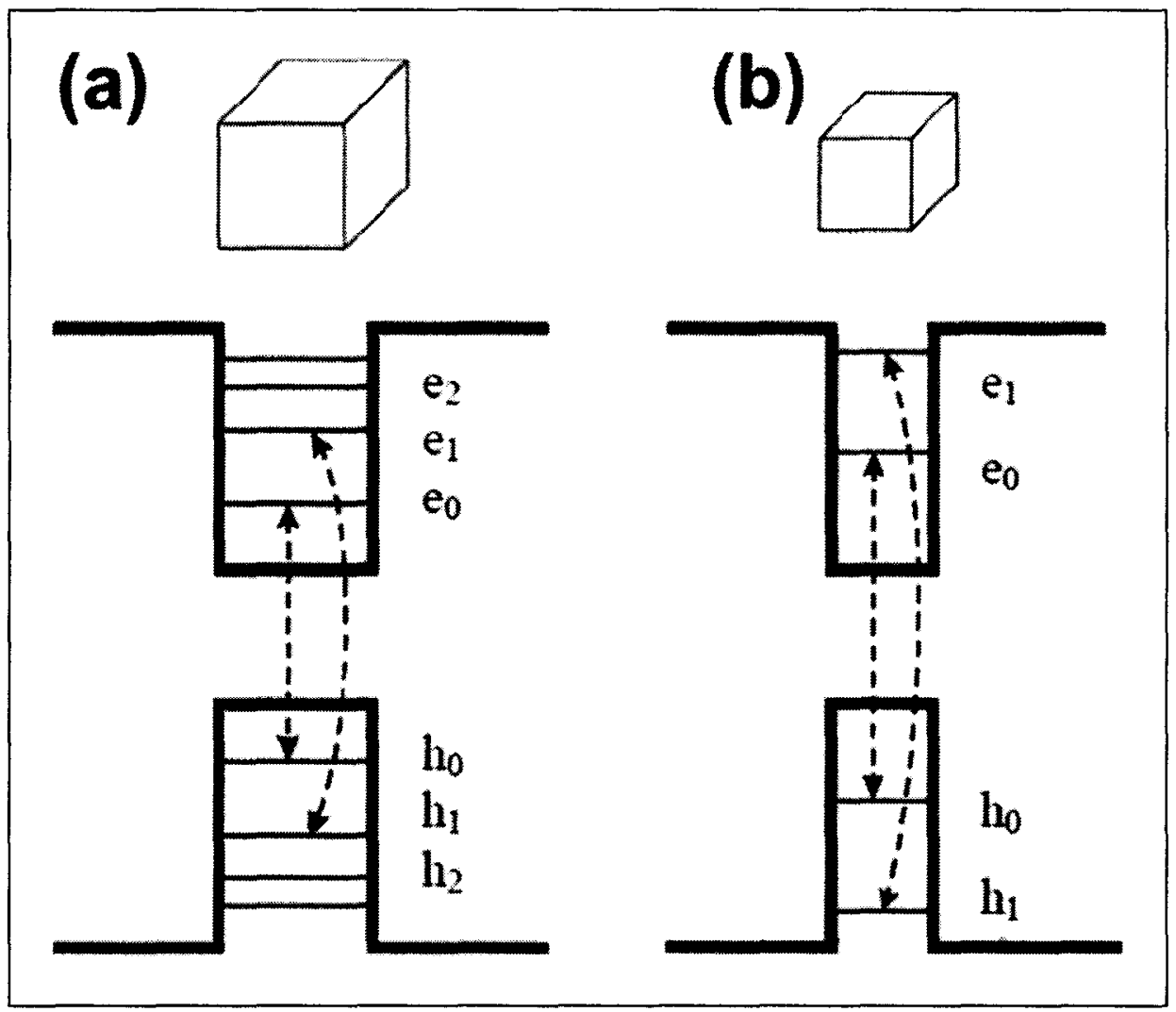

Figure 5.4 Comparison of discrete energy levels of two different QDs with (a) large radius and (b) small radius. As the size of the quantum dot decreases, the energy level spacing increases. ${ }^{128}$

However, the etching processes in the fabrication induced defects to the active QD material with limited attainable resolution. Several researchers have successfully 
fabricated QDs on substrates using epitaxial growth and selective growth metal organic chemical vapor deposition techniques. ${ }^{129-133}$ These techniques require complex growth control patterning with low density yield. One of the most widely successful and exploited epitaxial growth techniques for the fabrication of QDs is the StranskiKrastonow (SK) growth. The SK growth of the QDs is driven by the strain due to lattice mismatch which results in coherent, i.e. defect-free, three dimensional islands. ${ }^{134}$ The major limitation of this method is the cost of fabrication and the poor control over positioning of individual dots.

In this dissertation, QDs synthesized using solution chemistry (colloidal QDs) are discussed. Typically, colloidal suspensions of QDs are synthesized by introducing semiconductor precursors in the presence of semiconductor binding agents under thermodynamically favorable conditions for growing crystals. Several methods exist for the synthesis of colloidal QDs in solution such as synthesis from supersaturated glass solutions $^{135}$, via pyrolysis of organometallic precursors $^{136}$ and epitaxial growth technique. ${ }^{137}$ Colloidal QDs are generally made from group II and VI elements such as $\mathrm{CdSe}$ and $\mathrm{CdTe}$ along with zinc compounds $\mathrm{ZnO}, \mathrm{ZnSe}, \mathrm{ZnS}$ (used only as the shell material) or from group III and V elements such as InP and InAs. The size dependent properties of these QDs are striking when they are monodisperse in solution. Murray et al. first demonstrated the synthesis of $\mathrm{CdSe}, \mathrm{CdS}$ and $\mathrm{CdTe}$ QDs by injecting semiconductor precursors to a liquid coordinating solvent at high temperature. ${ }^{138}$ The prepared QDs were approximately 1.2 to 11.5 nanometers in diameter with good crystalline structure, surface derivatization and a high degree of monodispersity $(<5 \%$ root mean square in diameter). Ever since this seminal work, large efforts were devoted 
to the synthesis of QDs with precise control of the particle size, shape ${ }^{139-142}$ and the internal structure. ${ }^{143,144}$

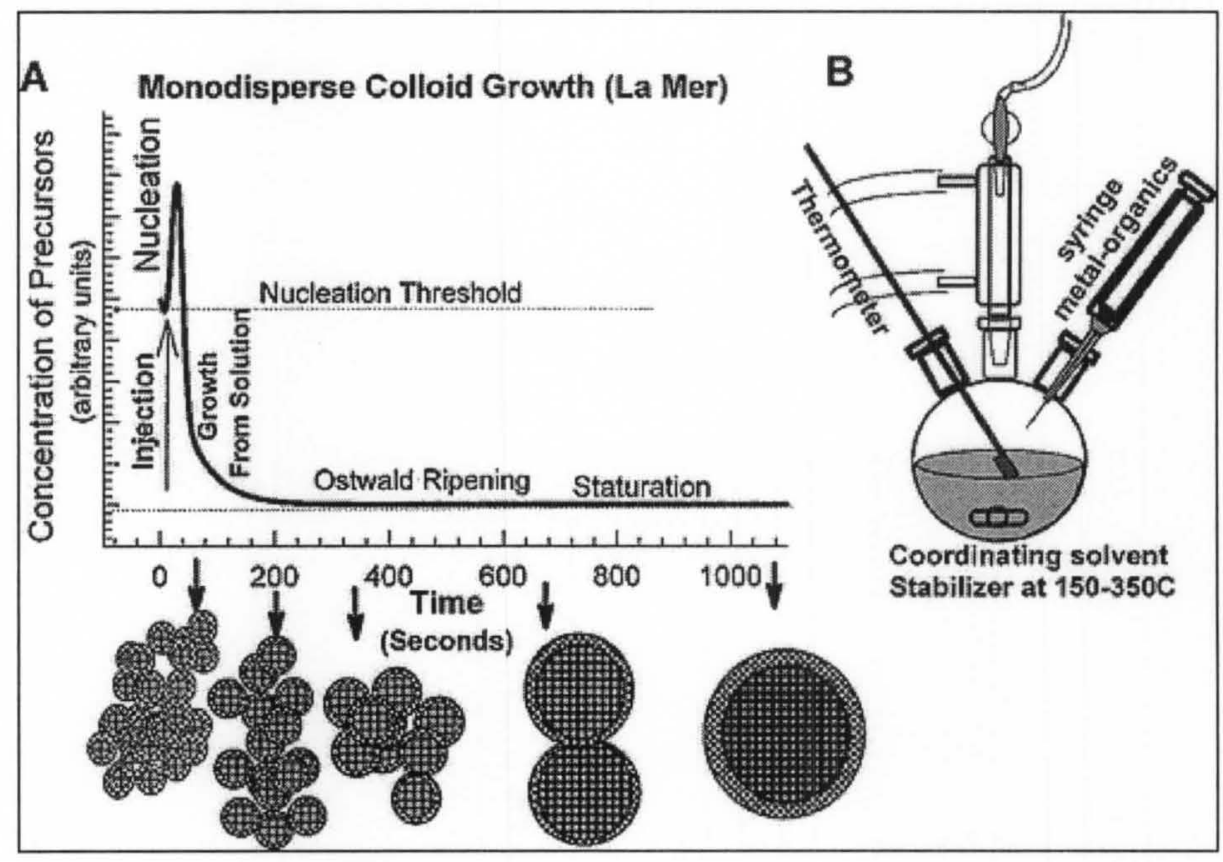

Figure 5.5 (A) La Mer model ${ }^{145}$ schematic of the different stages of nucleation and growth for the synthesis of monodisperse nanocrystals. As the nanocrystals grow with time, different sized crystals are isolated by periodically removing aliquots from the reaction chamber, (B). Typical apparatus used in the synthesis of monodisperse QDs samples. $^{111}$

In the $1950 \mathrm{~s}, \mathrm{La}$ Mer et al. proposed a model for the synthesis of monodisperse nanocrystals using a temporally discrete nucleation mechanism followed by slow controlled growth on the existing nuclei. ${ }^{145}$ When the precursors are rapidly injected into the reaction chamber, their concentration reaches above nucleation threshold as shown in Figure 5.5A. The resulting supersaturation is partially released by a short burst of nucleation. No new nuclei are created as long as the rate of decomposition of the 
precursors is not more than the rate of the addition of the precursors. The time for the formation and growth of the nuclei determine the initial size distribution of the nanocrystals. Monodispersity is achieved with time if the percentage of the nanocrystals during the nucleation event is small in comparison to the subsequent growth events. This phase is referred to as size-focusing point. In many systems, with time another growth phase called Ostwald ripening ${ }^{146,147}$ occurs in which smaller nanocrystals are dissolved due to their high surface energy while re-deposition occurs on the larger nanocrystals. This process dramatically simplifies the production of different sizes of nanocrystals by periodical removal of aliquots of the samples.

In the case of semiconductor QDs synthesis, metal-organic precursors are rapidly injected into a hot $\left(\sim 150-350^{\circ} \mathrm{C}\right)$ coordinating solvent (Figure 5.5B). The coordinating solvent consists of tri-n-octylphosphine oxide (TOPO) and tri-n-octylphosphine (TOP) long-chain alkylphosphine oxides and alkylphosphines which contain the basic functional groups to bind to the surface of the QD during growth preventing the growth of bulk materials. The alkyl chains (ligands) render stability to QDs and make them dispersible in many nonpolar solvents. In a typical CdSe QDs synthesis, dimethylcadimum and elemental selenium dissolved in liquid TOP are used as precursors. The room temperature precursors are rapidly injected into the reaction chamber containing boiling TOPO and stirred vigorously. Rapid, immediate nucleation and growth of CdSe QDs occurs as it is thermodynamically favorable because the concentrations of the precursors introduced are higher than the solubility of the resulting material. The growth of the QDs is kinetically controlled by the monomers diffusion as well as the reaction rate of the monomers at the QDs surface. High temperature helps in overcoming the steric, kinetic 
barrier required for the nucleation. Homogenous growth of the nuclei is achieved if the nucleation is stopped within a short period of time by a quick drop in the temperature and the monomer concentration. As per the La Mer model, the size distribution of the CdSe QDs widens after the size-focusing point and Ostwald ripening starts to occur. Aliquots of the reaction mixture are removed from the reaction chamber periodically to attain different sizes of CdSe QDs. The presence of the hot coordinating solvent yields in highly crystalline QDs with reduced lattice defects. The excess solvent and the byproducts of the reaction are removed from the aliquots by isolation and purification processes. ${ }^{138,148}$ Larger crystals present in the aliquots are precipitated using methanol and the resulting narrow size distributions of the CdSe QDs are re-dispersed in 1-butanol. The above mentioned method is the most effective and reproducible method for the synthesis of QDs composed of CdSe as well as other compositions. ${ }^{144,} 149,150$ Diverse precursors for CdSe QDs such as cadmium oxide, dimethylcadimium and cadmium acetate combined with TOP-Se and various coordinating solvents such as alkylamine, alkanoic acids are utilized to enhance the mono dispersity as well as the quantum yield. ${ }^{151-153}$

QDs prepared using the above mentioned synthesis procedure have large surface to volume ratios with a large surface fraction of the atoms exposed resulting in atomic or molecular orbitals that are not completely filled. The dangling bonds may bind with the organic ligands such as TOPO resulting in the passivation of the surface of the QDs that not only maintains the lattice structure but also protects the surface from external environment. ${ }^{110}$ However, the bond strength between the ligands and the surface is less than that of the semiconductor lattice which may result in desorption of the ligands 
making the core susceptible to external factors such as heat, UV light and ions. Thus in order to avoid damage to the core, it is desirable to grow a shell over the core QD surface, referred to as core/shell QDs. Several modifications of the core surface were investigated and more commonly inorganic coatings are used as the shell materials. The presence of the shell not only acts as a physical barrier for core degradation and prevents aggregation, but also results in stronger electronic insulation increasing the fluorescence efficiency. Passivation of the QDs core surface with a semiconductor shell did not seem to alter absorption and emission energies and the quantum yields as high as $50-70 \%$ were observed especially in the case of core/shell structures such as $\mathrm{CdSe} / \mathrm{ZnS}, \mathrm{CdSe} / \mathrm{CdS}$ and ZnSe/ZnS QDs. ${ }^{136,137,154}$ An additional shell over the core/shell structures were synthesized in which the middle shell provides strain relaxation between the inner core and the outer shell, further increasing the fluorescence efficiencies than the core/shell structures. Some of the core/shell/shell structures are $\mathrm{CdSe} / \mathrm{CdS} / \mathrm{ZnS}, \mathrm{CdSe} / \mathrm{ZnSe} / \mathrm{ZnS}$ and $\mathrm{CdSe} / \mathrm{ZnTe} / \mathrm{ZnS}$ QDs. ${ }^{155,156}$

The core/shell QDs are classified based on the band alignment between the core and shell semiconductor materials. In a type I structure, the shell is made of semiconductor material with a wider bandgap than the underlying core (Figure 5.6A). The conduction band edge and valence band edge of the core lies within the bandgap of shell material which confines the electrons and holes in the core regions where they recombine quickly. The shell in type I QDs passivates the core by physically separating it from the surrounding environment. Any change in the local environment has minimal effects on their optical properties. The fluorescence emission wavelength is slightly redshifted compared to the unmodified core QDs due to partial leakage of the exciton into 
the shell. The most commonly used type I QDs are $\mathrm{CdSe} / \mathrm{ZnS}, \mathrm{CdSe} / \mathrm{CdS}$ and InAs/CdSe core/shell systems. In the case of type II structure, the valence band edge or the conduction band edge of the shell material is located in the bandgap of the core. This staggered alignment of the bands results in the electrons and holes being spatially separated between the core and the shell. This results in longer lifetimes for the excitons before recombination occurs (Figure 5.6B, C).

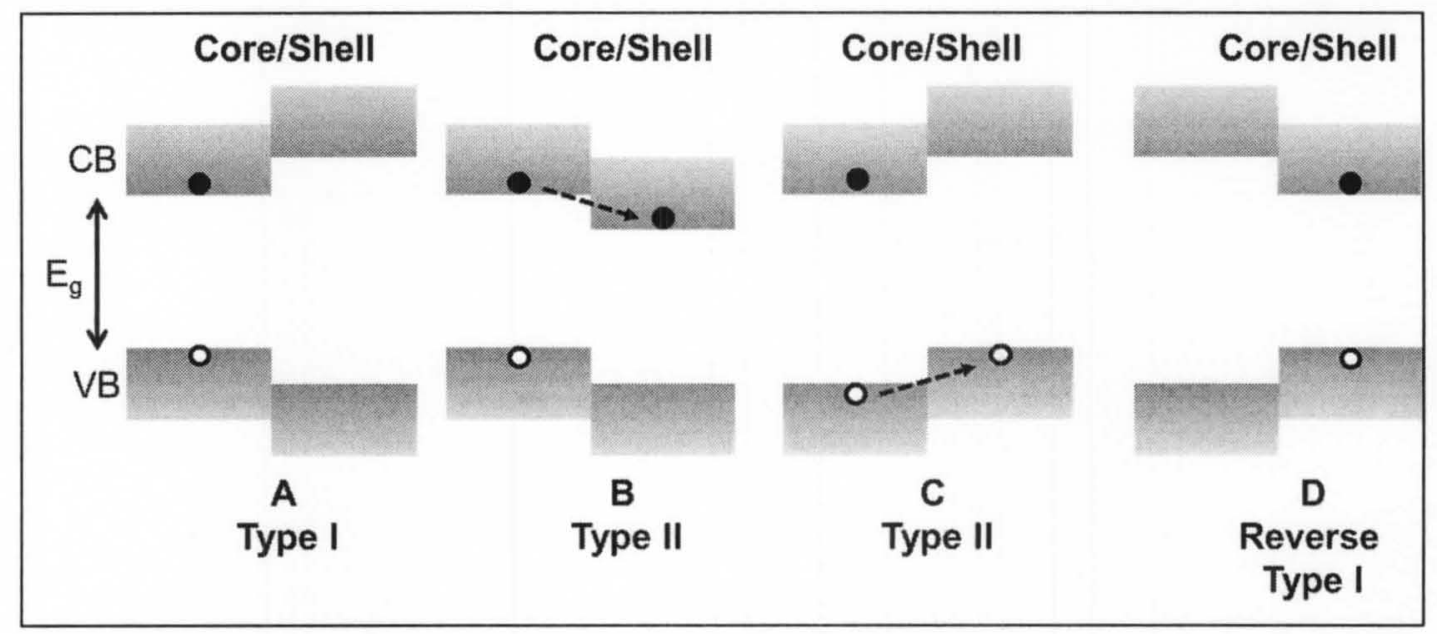

Figure 5.6 (A) Schematic of the energy band alignment of (A) type I, (B and C) type II and (D) Reverse type I core/shell QDs. CB and VB stands for conduction and valence bands respectively. Eg stands for bandgap of the semiconductor. The solid circle represents an electron and the empty circle a hole.

In the type II systems, the shell mainly contributes to the red-shift in the fluorescence emission. Some of the most commonly used type II QDs are CdSe/ZnTe, $\mathrm{CdTe} / \mathrm{CdSe}, \mathrm{CdS} / \mathrm{ZnSe}$ core/shell systems. ${ }^{157}$ Another variation of the type I core/shell system is the reverse type I core/shell QDs, in which a smaller bandgap shell is grown on a wider bandgap core material (Figure 5.6D). The charge carriers are partially delocalized in the shell material and the fluorescence emission can be tuned by varying the thickness 
of the shell. Some of the most widely studies systems are $\mathrm{CdS} / \mathrm{HgS}, \mathrm{CdS} / \mathrm{CdSe}$ and ZnSe/CdSe QDs. ${ }^{158}$

\subsubsection{Surface modification of QDs for biocompatibility}

As synthesized QDs are coated with hydrophobic molecules and hence are soluble in nonpolar organic solvents. It is necessary to solubilize QDs in aqueous solvents to render biocompatibility for biomedical applications. Two seminal studies successfully

demonstrated water soluble CdSe/ZnS core/shell QDs. ${ }^{159,}{ }^{160}$ In one of the studies done by Nie et al., as synthesized QDs were stabilized in aqueous solution using small thiolate ligands such as mercaptoacetic acid which binds to the zinc atom while the polar carboxyl groups render water solubility. The carboxyl groups are used to covalently attach biomolecules such as proteins, peptides and nucleic acids. Though these QDs are water soluble, the bonding between the ligand and the QDs is weak, causing rapid degradation in complex biological environments. In the other study done by Alivisatos et.al, core/shell QDs are modified with a third silica layer to make them water soluble. This method had advantages compared to the direct attachment to the QDs surface: as the multivalency of the extensively polymerized polysilane ensures the solubility of the QDs despite the potential loss of the thiol groups. In addition to this, the silica chemistry has been thoroughly investigated and the modified QDs retained fairly large quantum yield. More recently, several strategies were employed for the production of water soluble $\mathrm{QDs}^{161}$. A representative list of the various strategies for capping and dispersing QDs is shown in Figure 5.7. One of the methods involves exchanging the native TOP/TOPO ligands with bi-functional ligands, referred to as cap exchange method. The bi-functional ligands consists of hydrophobic groups at one end like the thiols $(-\mathrm{S}-\mathrm{H})$ which bind to the 
surface of the QDs while hydrophilic groups at the other end like the carboxyl groups ($\mathrm{COOH})$ that render water solubility. ${ }^{162-165}$ For example, CdSe/ZnS QDs are coated with mercaptoacetic acid and trimethylsiloxane. However, the ligand exchange methods reduced the fluorescence efficiency of the QDs with a potential to cause aggregation. More recently, these problems were overcome by preserving the native TOP/TOPO ligands and covering the hydrophobic QDs with amphiphilic polymers. ${ }^{166-169}$ Another strategy involves using silica shells functionalized with polar surface groups to protect the hydrophilic QDs. ${ }^{170}$ The presence of such amphiphilic polymer coatings (silica shells) increases the size of the QDs, for example in the case of CdSe/ZnS QDs from $\sim 4-8 \mathrm{~nm}$ before coating to $\sim 20-30 \mathrm{~nm}$. QDs are purified from the residual ligands and excess polymers via ultracentrifugation, dialysis or filtration methods prior to their use in biological applications.

\subsection{BIOMEDICAL APPLICATIONS OF ODS}

\subsubsection{Advantages of QDs}

Even though QDs are developed for electronics and optics approximately two decades ago, their potential for biomedical applications was realized only recently. The ability to synthesize water soluble QDs opened up a plethora of applications for their use in biology and medicine. Following the two seminal reports ${ }^{159,160}$ demonstrating the use of QDs as biological labels, large research efforts were devoted to the development of a variety of applications for QDs. The unique optical properties of the QDs make them promising candidates for in vitro as well as in vivo applications. 


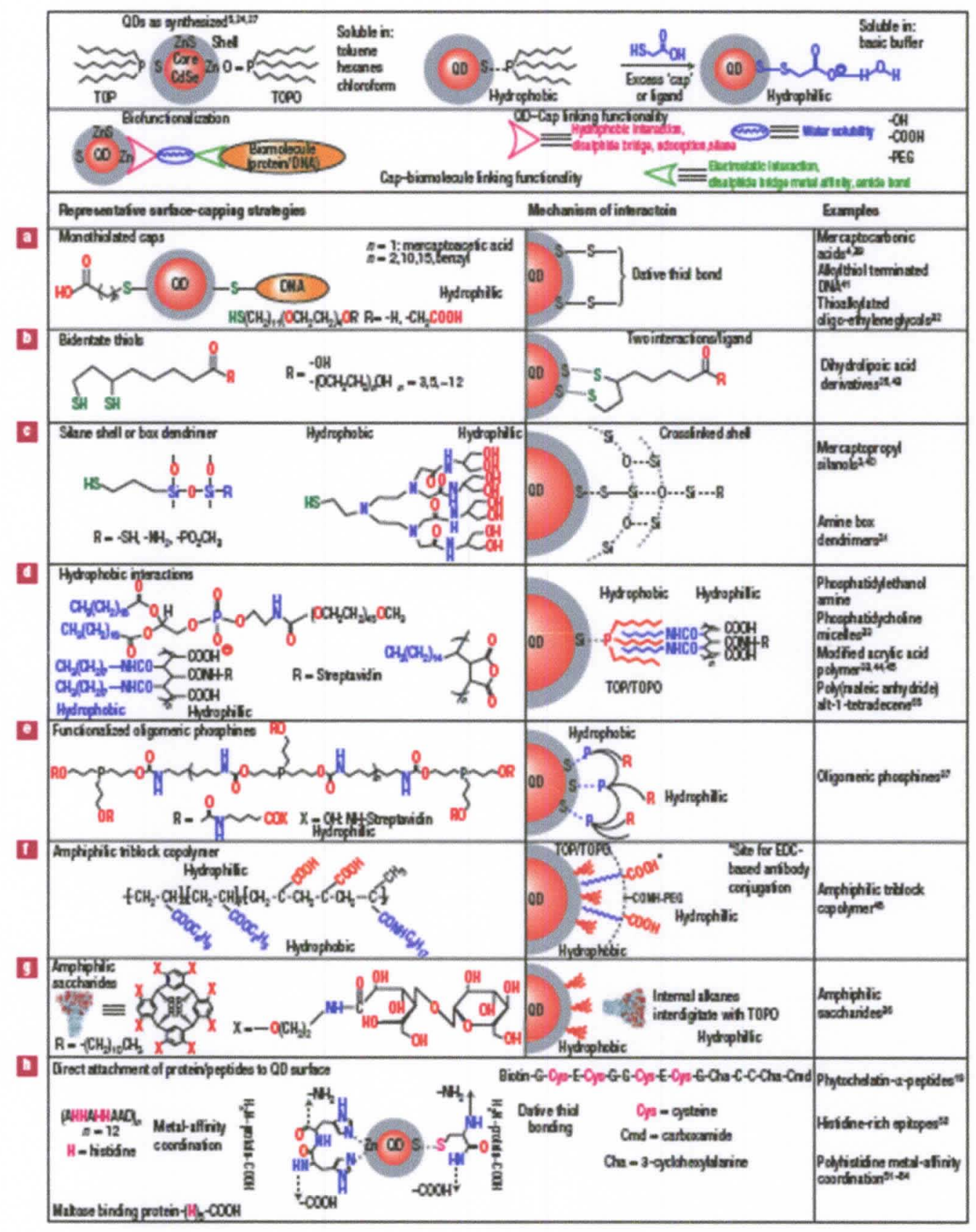

Figure 5.7 Schematic of the strategies employed for solubilization and biofunctionalization of QDs (a-h). ${ }^{161}$ 
QDs offer a number of advantages over traditional biological labels such as fluorphores or organic dyes. The fluorescence emission is tunable based on size as well as composition from the ultraviolet to the visible and near-infrared wavelengths. For example, in the case of CdSe QDs, the fluorescence emission wavelength changes from $475 \mathrm{~nm}$ for $2.1 \mathrm{~nm}$ diameter to $650 \mathrm{~nm}$ for a $7.5 \mathrm{~nm}$ diameter (Figure 5.8).

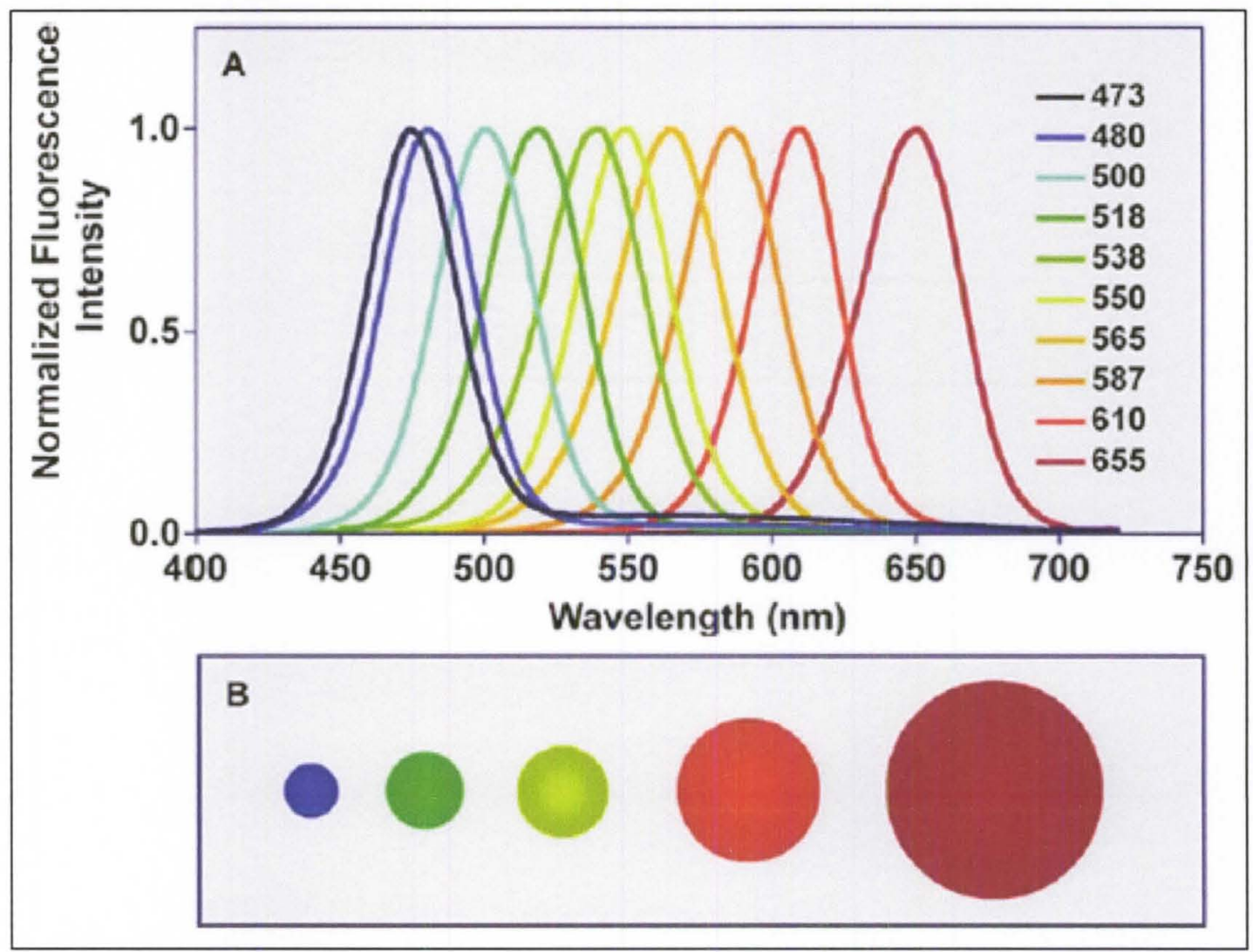

Figure 5.8 (A) Size tunable fluorescence emission of CdSe QDs, (B) relative sizes of QDs with diameters of $2.1 \mathrm{~nm}, 2.5 \mathrm{~nm}, 2.9 \mathrm{~nm}, 4.7 \mathrm{~nm}$ and $7.5 \mathrm{~nm}{ }^{171}$

The composition of QDs is modified to tune the fluorescence emission from ultraviolet wavelengths as in the case of $\mathrm{ZnS}$ QDs to near infrared wavelengths in the case of PbSe or PbTe QDs. Thus, the fluorescence emission can be also be tuned based on the biological applications as shown in Figure 5.9. 
QDs have very narrow and symmetric fluorescence emission spectrum (20-30 nanometers, full width half maximum, FWHM) compared to organic dyes and fluorescence proteins which have significant red-shift tails. QDs have broad absorption bands with high molar extinction coefficients (approximately $10^{5}-10^{7} \mathrm{M}^{-1} \mathrm{~cm}^{-1}$ ) which is 10-100 times larger than that of organic dyes ${ }^{172}$ and large effective stokes shifts (Figure 5.10). This provides the ability to excite a family of different colored QDs with a single excitation wavelength, thereby providing multiplexing capability.

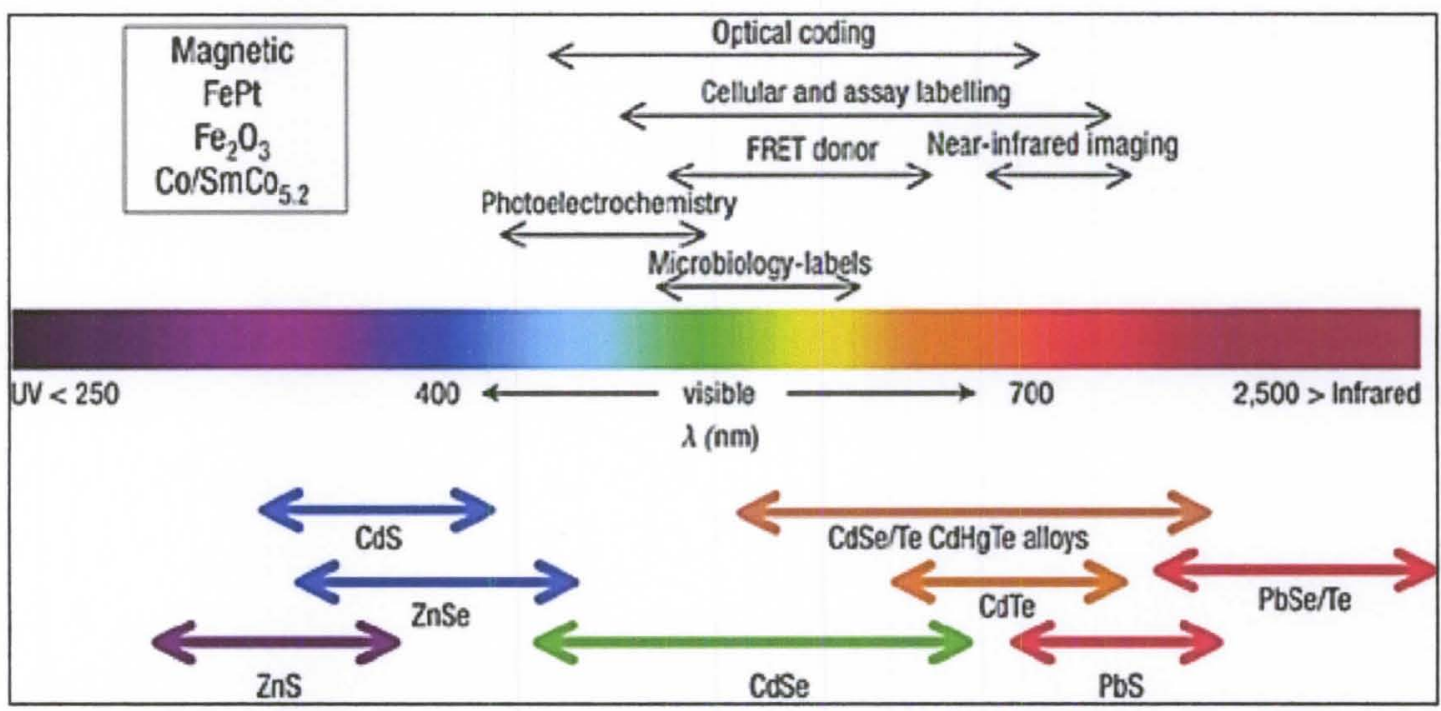

Figure 5.9 QDs composition scaled as a function of the fluorescence emission wavelengths from ultraviolet to near infrared wavelengths. ${ }^{161}$

The large stokes shifts in QDs not only increases the detection sensitivity but also help in differentiating them in biological samples with high auto fluorescence backgrounds. In addition to the mentioned advantages, QDs have high resistance to photobleaching as well as exceptional resistance chemical degradation. Under similar excitation, QDs are at least 1000 times more photostable than organic dyes such as Texas 
Red (Figure 5.11). Thus QDs are excellent candidates for tracking based studies over a long period of time.

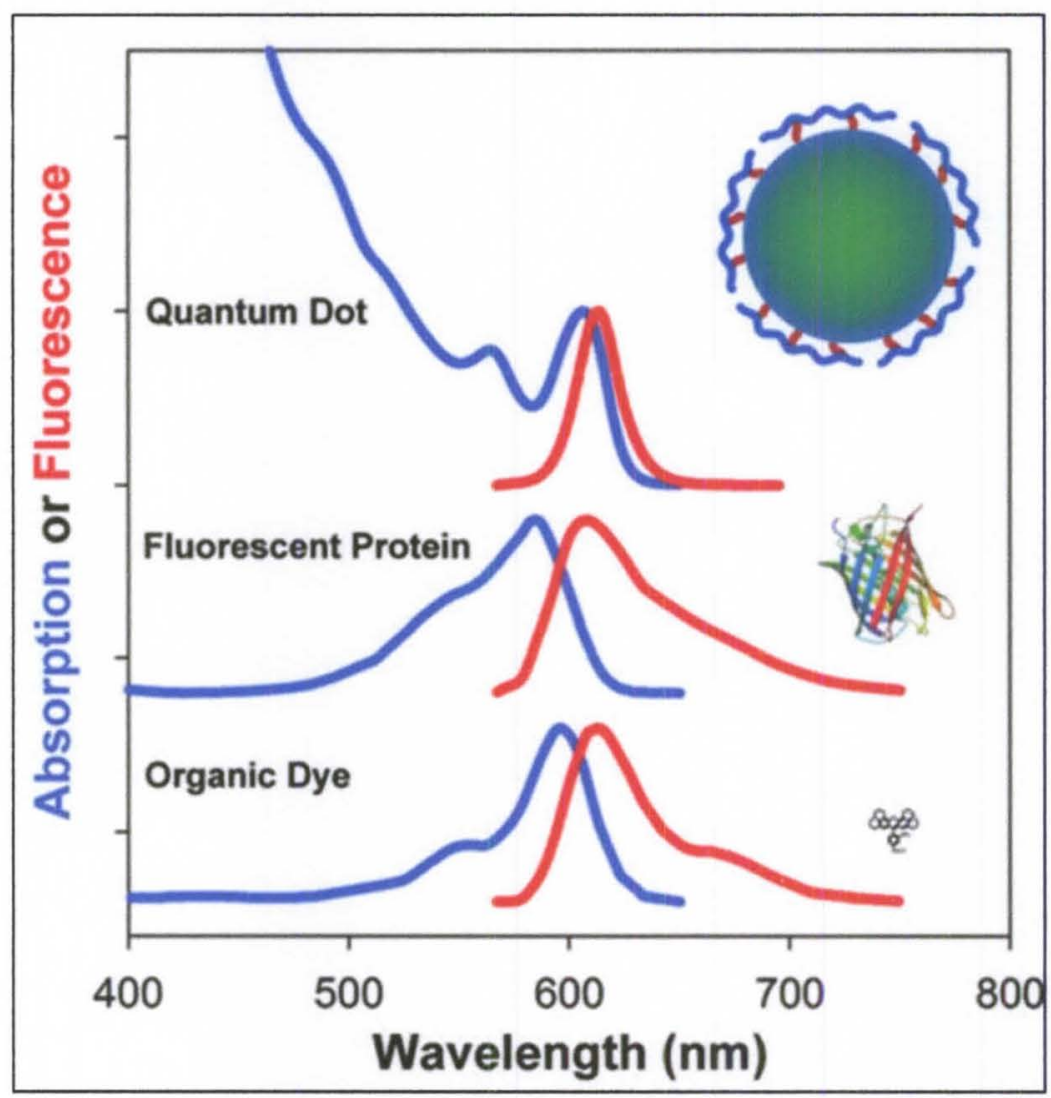

Figure 5.10 Comparison of the absorption (blue) and fluorescence spectra (red) of CdSe QDs with fluorescence proteins (mCherry) and organic dye (Texas red). The absorption spectrum of QDs is broad and the emission spectrum is symmetric and narrow. ${ }^{173}$

\subsubsection{Bioconjugation of QDs}

Apart from the advantages, the comparable size of the QDs with many biological systems such as proteins, antibodies and nucleic acids have led to their extensive use in various biomedical applications. Water soluble QDs are conjugated with many biomolecules like proteins, antibodies and other targeting or affinity ligands. 


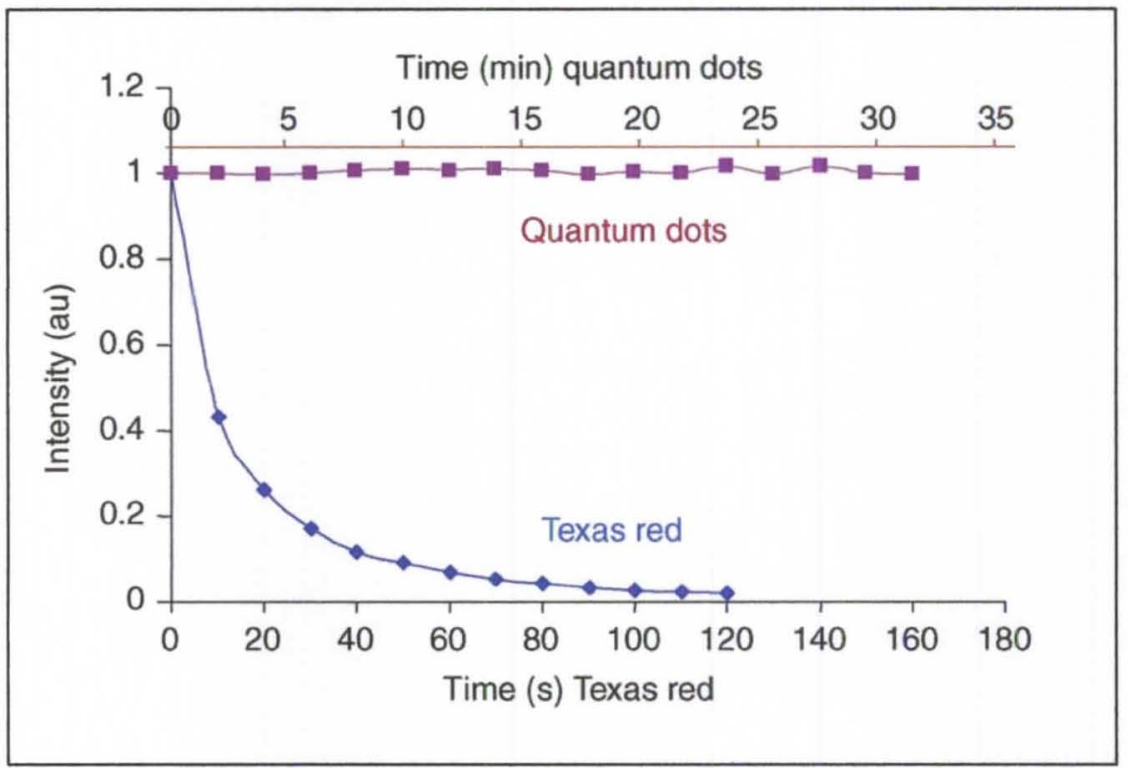

Figure 5.11 Comparison of the photobleaching properties of QDs and organic dye (Texas Red) under similar excitation conditions. ${ }^{174}$

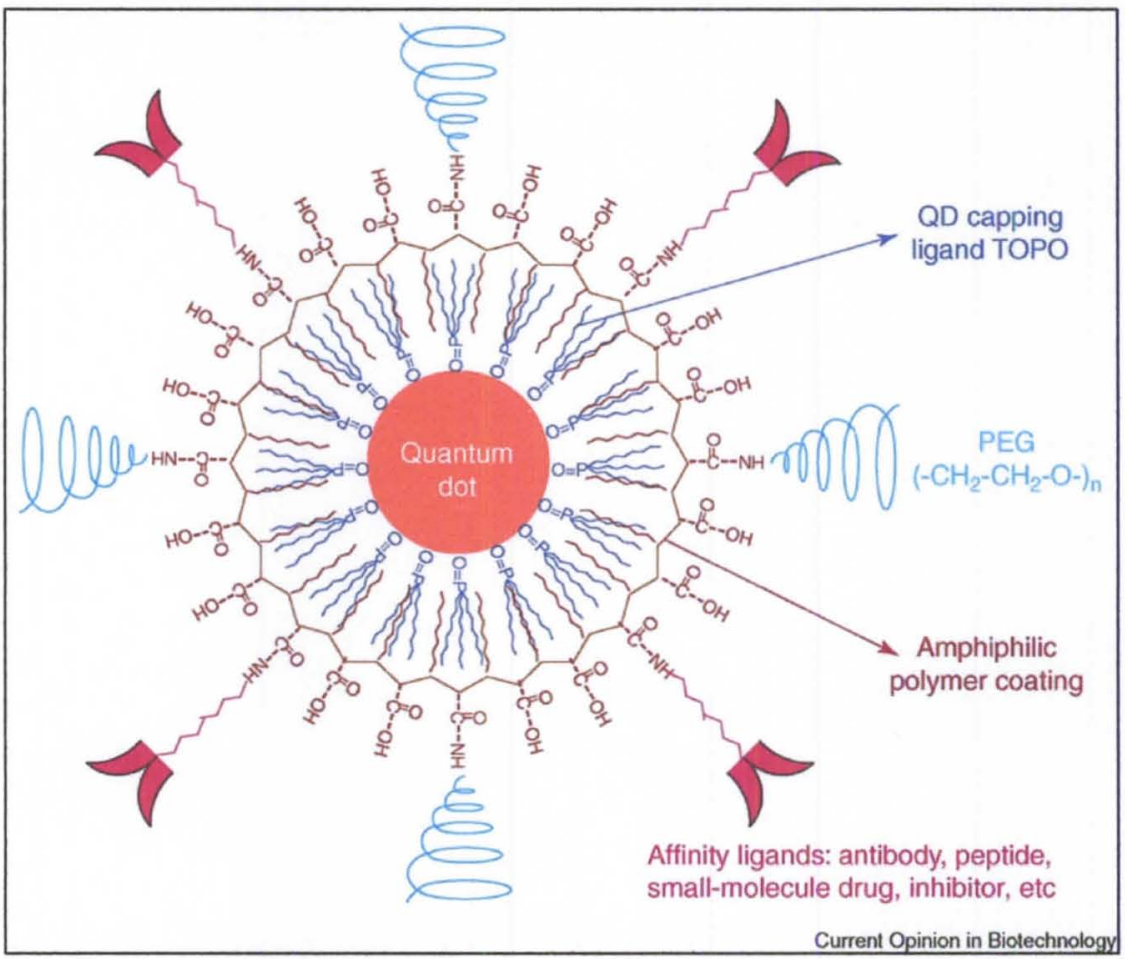

Figure 5.12 Schematic of QDs as multifunctional biomolecule hybrid. ${ }^{174}$ 
The QDs: biomolecule conjugates possess the unique spectroscopic properties of the QDs as well as retain the functional properties of the attached biomolecule. A single QD can be conjugated to multiple proteins or biomolecules resulting in a multifunctional nanoparticle-biomolecule hybrid (Figure 5.12). Bioconjugation of QDs is achieved using standard coupling reactions like passive adsorption, multivalent chelation or through formation of covalent bonds. Two of the most popular crosslinking reactions are based on amide bond formation using EDAC (ethyl-3-dimethyl amino propyl carbodiimide) and amine-sulfhydryl coupling using SMCC (succinimidyl-4-N-maleimidomethylcyclohexane carboxylate). ${ }^{175}$

In EDAC method, QDs containing carboxylate groups are coupled with the amine groups present of the biomolecules using a single or two step reactions (Figure 5.13a). In the single step process, the QDs are activated using EDAC in the presence of amine containing molecule like proteins. As the proteins contain both the carboxylates as well as amines, polymerization can occur. A two-step reaction is used to minimize this polymerization problem. In the first step, EDAC is used in the presence of sulfo-NHS (Nhydroxysulfosuccinimide) which converts the carboxyl groups on the QDs to intermediate amine-reactive sulfo-NHS esters. Following a quick separation step to remove the unreactive or excess reactants, the activated QDs are mixed with the protein solution in a desired molar ratio. This results in amide bond formation without the polymerization of the protein in the solution. The presence of sulfo-NHS increases the efficiency of EDAC mediated coupling. The second method for QDs bioconjugation is based on using sulfo-SMCC, which is a heterobifunctional crosslinking agent with an amine-reactive NHS ester group at one end and a thiol-reactive maleimide group at the 
other end. Amine containing QDs are activated by converting amines to sulfhydrylreactive maleimides using $\mathrm{SMCC}$ which then reacts with thiol-containing proteins or other biomolecules (Figure 5.13b). The advantage of the EDAC method is that most of the proteins and biomolecules contain primary amines and carboxyl groups and hence do not need any further chemical modifications for conjugation. The SMCC method is very useful in the case of biomolecules which have the free sulfhydryl groups to react with the amine groups on the QDs. These two methods are used interchangeably depending on the availability of the reactive chemical groups. The QDs surface is also modified with hydrophilic molecules like polyethylene glycol to minimize nonspecific binding or to decrease the clearance rate from the blood stream for in vivo applications.

In another method of QDs bioconjugation, Goldman et al. demonstrated the use of an adaptor protein for immunoglobulin $\mathrm{G}$ antibody coupling. ${ }^{176}$ The conjugation method utilizes an engineered adaptor protein which has a positively charger leucine zipper moiety which links to the QDs (using electrostatic or hydrophobic self-assembly) and a protein $\mathrm{G}$ domain that binds to the antibody $\mathrm{Fc}$ region. The antibody $\mathrm{Fc}$ region is effectively connected to the surface of the QD while the antigen specific $\left.\mathrm{F}(\mathrm{ab})^{\prime}\right)_{2}$ domain is free to bind to the specific target (Figure 5.12c). A chelating compound like Ni-NTA (nickel-nitrilotriacetic acid) is also used to bind to the 6-histadine containing biomolecules. ${ }^{174}$ This method is more advantageous as it provides control over the molar ratios, the orientation of the biomolecules as well as improves the binding efficiency.

Even though the QDs bioconjugation methods involve standard coupling reactions, some difficulties still exists. Firstly, the buffer and salt compatibility needs to be taken into account when dealing with QDs. Smaller charged particles typically 
maintain stability in colloidal dispersion by like-charge repulsion which effectively prevents aggregation by Vander Waals or hydrophobic interactions. Precipitation will occur if the salts or buffers neutralize the QDs surface charges. It is also important to consider the optimal number of biomolecules conjugated to each QD. In the case of antibody-QDs conjugates, two to three antibodies conjugated per QD may be sufficient while for protein-QDs conjugates, higher ratio of protein to QDs may be necessary. Most importantly, the correct orientation of the biomolecules, for example, antibodies on the QDs is necessary. Otherwise the antibodies may not be functional which results in low avidity.

\subsubsection{Applications of QDs}

One of the most attractive biomedical applications of QDs is in labeling of cells and tissues. Several reports have published in using bioconjugated QDs for in vitro as well as in vivo applications. Chan et al. was one the first to demonstrate the use of IgG conjugated QDs for labeling of HeLa cells. ${ }^{159}$ The QDs: IgG conjugates were detected by polyclonal anti-IgG and the conjugates were readily uptaken by the cells. In another study by Mattoussi et al., a chimeric fusion protein was designed which electrostatically bound

to the oppositely charged surface of the QDs. ${ }^{162}$ QDs conjugated to maltose binding protein-basic leucine zipper (MPB-zp) fusion protein were used to detect the interactions between the MPB and amylose affinity resin. The addition of free maltose reversed the binding. The QDs:MPB-zp conjugates preserved the bioactivity of the MPB domain despite the use of a fusion protein. 


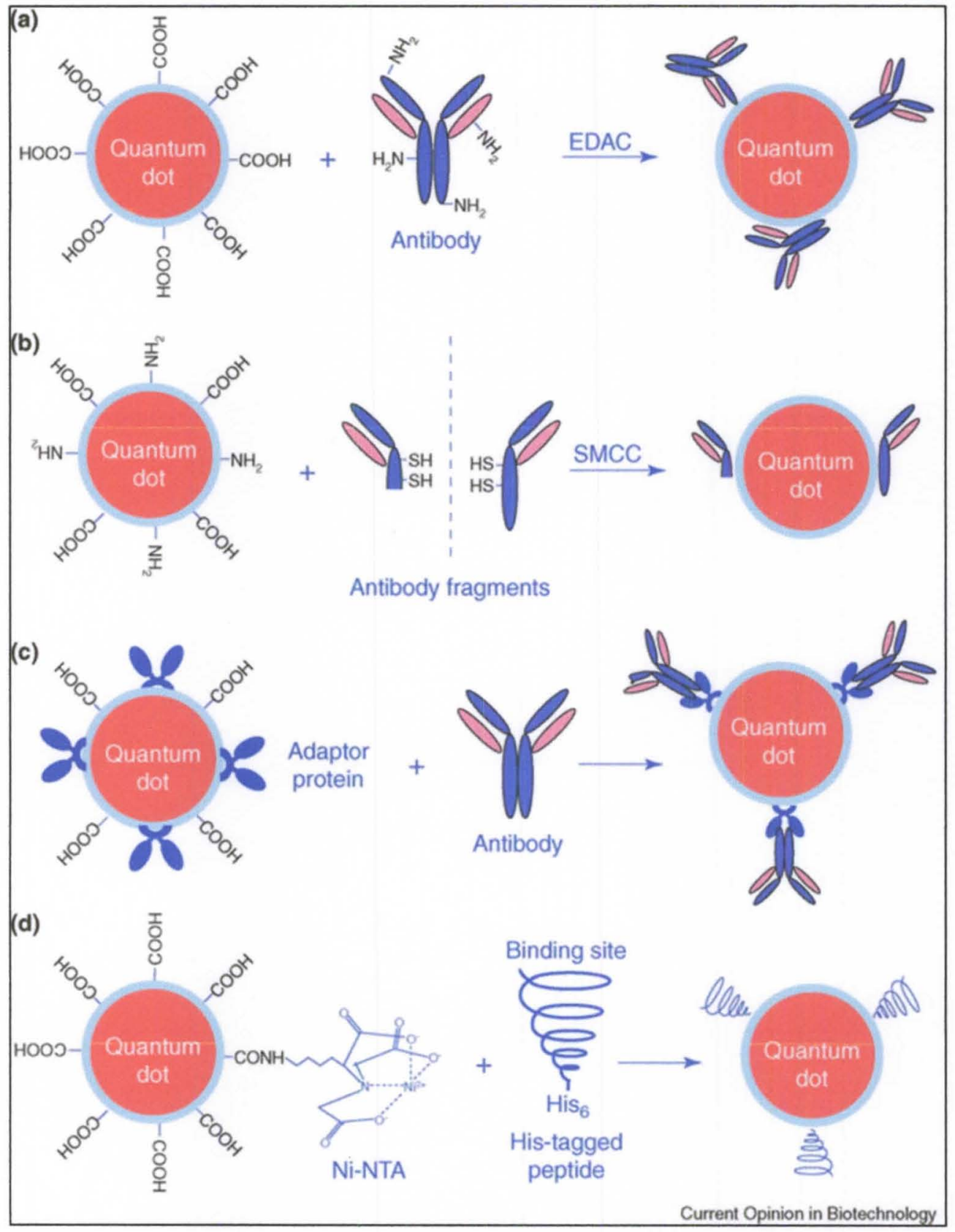

Figure 5.13 Typical methods of QDs bioconjugation. (a) Covalent crosslinking of antibody with QDs using EDAC chemistry method, (b) conjugation of antibody fragments using SMCC method, (c) conjugation of QDs using adaptor protein method and (d) conjugation of Ni-NTA modified QDs with histidine tagged peptides and biomolecules. ${ }^{174}$ 
QDs were conjugated with DNA molecules and QD:DNA arrays were generated which self-assemble spontaneously. ${ }^{177,}{ }^{178}$ Pathak et al. used QDs to detect the hybridization of DNA to complimentary sequences. ${ }^{179}$ In this study, QDs were conjugated with two separate oligonucleotides, one having a known complimentary sequence present only in human $\mathrm{Y}$ chromosome and another random sequence with no known complimentary sequence. When these QDs:DNA conjugates were incubated with human sperm cells, strong fluorescence signal was observed in the cells with $\mathrm{Y}$ chromosome indicating successful hybridization of the DNA. However, minimal or no signal was observed with the one labeled with random sequence indicating low nonspecific hybridization. QDs conjugated to $\operatorname{lgG}$ and streptavidin were used to label breast cancer marker, Her2, on the surface of cancer cells. Other cellular targets like actin and microtubule fibers in the cytoplasm, nuclear antigens were also labeled using specific QDs bioconjugates. ${ }^{167}$

QDs were also used to track motility and migration of cells. Cancer cells uptake QDs and leave behind a fluorescence free phagokinetic tail during their migration. ${ }^{180}$ The photobleaching resistance property of QDs was exploited by Dahan et al., in which individual glycine receptors in neuronal cells were tracked using single QDs. ${ }^{181}$ Micelle stabilized QDs were used as cell lineage tracers by injecting into Xenopus embryos. ${ }^{166}$ In this study, it was demonstrated that the QDs are stable in vivo and non-toxic and their strong fluorescence could be followed to the tadpole stage. The large stokes shift in the QDs was mainly exploited in many in vivo applications as it overcomes the problems of auto fluorescence from the tissue. Near infrared QDs with emission $\sim 840 \mathrm{~nm}$ were synthesized which achieved deeper penetration than the currently available near infrared 
dyes. ${ }^{182,183}$ Kim et al. demonstrated the use of the near infrared QDs in bioimaging by applying them to sentinel lymph node-mapping in cancer surgery of large animals. ${ }^{184}$ QDs were also used for tracking of tumor cells in vivo during metastasis by conjugating to tumor targeting antibodies. ${ }^{169,185}$ In vivo studies performed in mice with human cancer cells showed that the QDs conjugates accumulate at the tumor sites when tracked using multiphoton microscopy. Thus QDs provide the ability for cell tracking in vivo without compromising or sacrificing the animals which is an enormous improvement in comparison to current techniques. Bioconjugated QDs were also used for assays like micro-nanaoarrays, FRET and ELISA for the detection of specific clinical markers. ${ }^{186-188}$

\subsection{Toxicity of QDs}

Most of the commonly used QDs are made of toxic heavy metal atoms like $\mathrm{Cd}$, $\mathrm{Hg}, \mathrm{Pb}, \mathrm{As}$ and the like. CdSe QDs contains the divalent cadmium which is a nephrotoxin in its ionic form. Even though they are embedded in a biological unreactive $\mathrm{ZnS}$ shell and a polymer coating, the toxicity of these QDs is not very well understood. As the toxicity due to $\mathrm{Cd}^{+2}$ is well reported, large amount of research was focused on the release of free cadmium ions into the cells. Cadmium ions released can bind to sulfhydryl groups present in many biomolecules like proteins which causes decrease in the functionality of various subcellular organelles. Yang et al. assessed the tissue disposition and pharmacokinetics of QDs in mice after a single intravenous injection for up to 28 days by measuring the cadmium levels using inductively coupled plasma-mass spectrometry (ICP-MS). ${ }^{189}$ Increased levels of cadmium were observed in the liver and kidneys indicating the degradation of QDs over time. However, it was not apparent whether the cadmium is in free form or in nanocrystalline form. Several other groups have 
investigated the release of $\mathrm{Cd}^{+2}$ and have observed that its release correlates with the cytotoxic indications. ${ }^{190,191}$ When CdSe QDs were exposed to air or UV light, oxidative release of $\mathrm{Cd}^{+2}$ was observed. However, coating these QDs with $\mathrm{ZnS}$ shells virtually minimized the toxicity indicating that the shell protects the release of the cadmium. ${ }^{192}$ More recently, greener chemistry based and non-cadmium based QDs were synthesized for reduced toxicity in biomedical applications. ${ }^{193,} 194$ Despite several studies, it is difficult to conclude about the toxicity due to the variation in the synthesis, surface coatings of the QDs and the experimental conditions like the cells under investigation, time of exposure and concentrations used.

\subsection{ODs IN THIS DISSERTATION}

In this dissertation, QDs are used for cellular labeling and imaging of biomolecules. Commercially available $\mathrm{CdSe} / \mathrm{ZnS}$ core/shell QDs are purchased from Ocean Nanotech, LLC. They have fluorescence emission $\sim 530 \mathrm{~nm}$, hydrodynamic size of $\sim 26 \pm 6.07 \mathrm{~nm}$ and a zeta potential around $-43.05 \mathrm{mV}$. As purchased QDs are coated with amphiphilic polymer and are dispersed in aqueous borate buffer solutions with reactive carboxyl groups. The organic layers consist of a monolayer of oleic acid/octadecylamine and a monolayer of amphiphilic polymer with the thickness of the total organic layers $\sim 4 \mathrm{~nm}$.

The ability to image QDs in cells for extended periods of time helps in studying living cells and tissue in real time. The motivation for the use of QDs in this dissertation is to study the activity of an important intracellular protein- NF-kB (Nuclear Factor kappa-light chain-enhancer of activated B cells). NF-kB consists of family of proteins 
that play an important role in many biological processes such as inducing immune and inflammatory responses, developmental processes, cellular growth and cell death or apoptosis. Traditionally, the activity of this particular protein is assessed by performing assays like immunofluorescence using fluorescent labeled antibodies. However, this approach has a number of disadvantages. Firstly, the antibodies are fluorescently labeled using organic dyes like FITC or Texas Red which photobleach very quickly and have low signal-to-noise ratio over time. The assays performed require the cells to be fixed to study the activity of the protein at a particular time point. QDs not only provide strong fluorescence with high signal-to-noise ratio but also significantly improve the real-time detection of the protein.

The use of QDs in this dissertation is divided into two sections. In the first section, novel technique for the intracellular delivery of QDs is demonstrated. In the next section, QDs are conjugated to antibodies of the NF-kB protein and preliminary results showing the ability of antibody conjugated QDs to label the protein is presented. 


\section{CHAPTER VI - REVERSIBLE PERMEABILIZATION FOR INTRACELLULAR DELIVERY OF QDS}

\subsection{PURPOSE OF THE STUDY}

The motivation for the use of QDs in this dissertation is to utilize their unique ability to image intracellular biomolecules like proteins over extended periods of time. In this dissertation, QDs are conjugated to antibody specific to an important functional protein- NF-kB (Nuclear Factor kappa-light chain-enhancer of activated B cells). It is a nuclear transcriptional factor consisting of 5 subunits- p65/RelA, p50/p105, p52/p100, RelB, and c-Rel, that form homo- or heterodimers. ${ }^{195-197}$ The classic form of NF-kB family is the heterodimer of the p65 and p50 subunits. NF-kB plays an important role in many biological processes like inducing immune and inflammatory responses, cell developmental processes, cellular growth and cell death or apoptosis. ${ }^{198}$

In unstimulated cells NF-kB dimers (p65/p50) are sequestered in the cytosol as they are bound to an inhibitory complex, IkB. The IkB inhibitory protein masks the recognition of the nuclear localization signal (NLS) present within the NF-kB sequence and thus restricts the NF-kB in the cytoplasm. Upon cell stimulation, IkB kinase (IKK) complex gets activated that leads to the phosphorylation and ubiquitination of $\mathrm{IkB}$. This degradation of $\mathrm{IkB}$ allows the release of $\mathrm{NFkB}$ which is free to translocate into the 
nucleus. In the nucleus, NF-kB binds to specific DNA elements and activates the transcription of genes. NF-kB activates to various external stimuli including bacterial products, viruses, stress (oxidative, physiological or physical), inflammatory cytokines and environmental hazards such as radiation. ${ }^{199-201}$ (Figure 6.1) It is also reported that abnormal and constitutive activation of NF-kB is associated with many chronic human diseases including arthritis, asthma, cancer, diabetes, stroke and AIDS. ${ }^{202,}{ }^{203}$ Thus inhibition of NF-kB activation is an emerging theme in the design of anti-inflammation therapies as it is clinically relevant for the treatment of diseases that involve inflammation. ${ }^{204-206}$ Several studies reported that the pathophysiological role of NF-kB can be either protective or detrimental and significance of the type of cells is also not very well understood. Hamid et al investigated the role of NF-kB in heart failure and demonstrated that chronic activation of NF-kB p65 in myocytes exacerbates cardiac remodeling and is pro-apoptotic. ${ }^{207}$ The presence of NF-kB p65 and p50 is studied using techniques like immunohistochemistry, ELISA and western blots which involve fixing the cells at particular time point. However, these techniques are not only laborious but also time consuming. QDs are excellent candidates to observe the dynamics of the activity of NF-kB in real time in live cells.

In this dissertation, QDs are conjugated with antibodies specific to NF-kB p65 subunit and their bright fluorescence will be used to image the nuclear translocation of NF-kB upon cell stimulation. H9c2 cells, rat cardiomyocyte cell line, are used as representative model to test the nuclear translocation of NF-kB p65. 


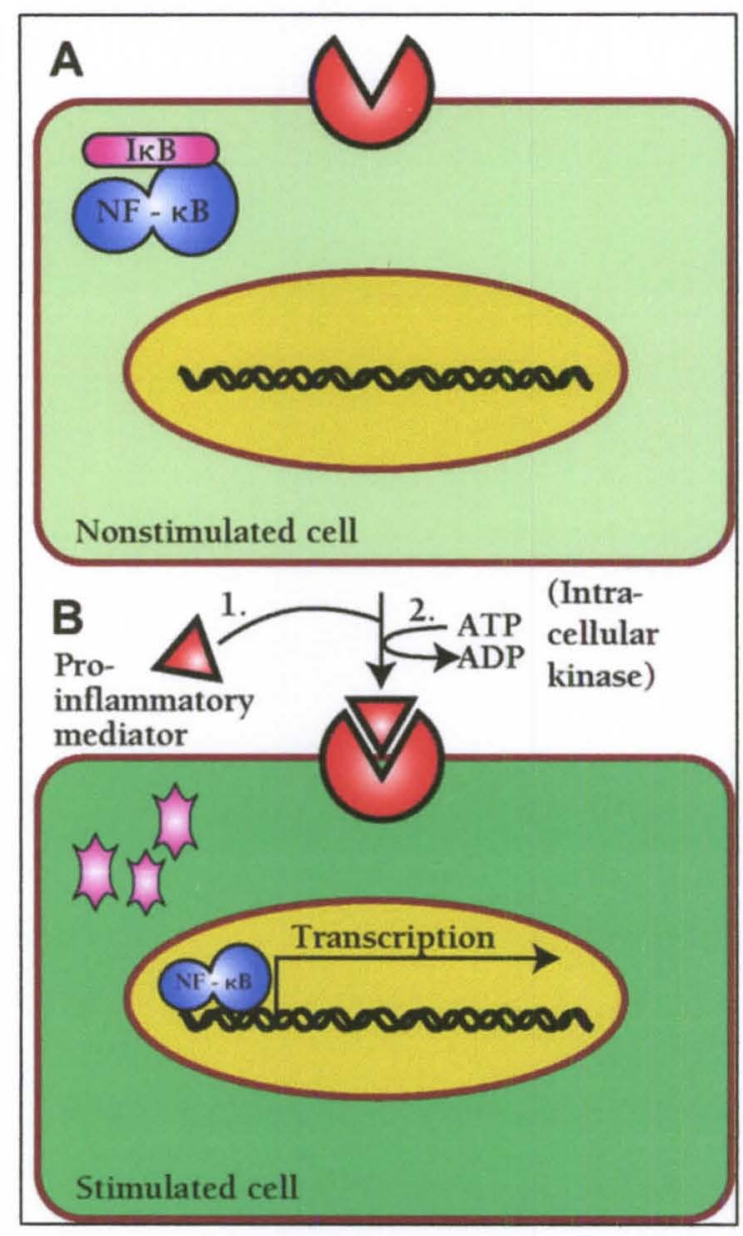

Figure 6.1 Schematic showing the activity of NF-kB. (A) In unstimulated cell NF-kB is inactive as it is bound to inhibitory protein $\mathrm{IkB}$, (B) whereas in stimulated cell, proinflammatory stimuli activates specific protein kinase resulting in the degradation of $\mathrm{IkB}$ and nuclear translocation of NF-kB. ${ }^{204}$

H9c2 cells are stimulated with different external stimuli like TNF- $\alpha$ (tumor necrosis factor), UV radiation and bacterial LPS (lipopolysaccharide) and QDs: p65 NF$\mathrm{kB}$ conjugates are used to detect the presence of NF-kB protein. The bioconjugation of QDs with NF-kB p65 antibodies and the experimental conditions are discussed in detail in the subsequent sections. 


\subsection{INTRACELLULAR DELIVERY OF QDS}

Prior to imaging nuclear translocation of NF-kB using QDs, intracellular delivery of freely dispersed QDs: NF-kB conjugates needs to be accomplished. Traditional bimolecular delivery methods utilize plasmids, cationic polymers, lipids, and viruses which have inherent disadvantages such as degradation in physiological solutions and need for complex conjugation techniques. ${ }^{208,209,210}$ QDs have high chemical and biochemical stability and their small size and large surface area allows simultaneous conjugation with multiple biomolecules making them highly desirable as imaging agents and biomolecular delivery vehicles for biomedical applications. ${ }^{160}$ Typically, the QDs were dispersed in aqueous buffer solutions and have been successfully functionalized with various biomolecules like DNA, proteins and antibodies for delivery applications. ${ }^{159}$ This chapter reviews the current methods for the intracellular delivery of QDs. A novel technique for efficient intracellular delivery of QDs will be discussed along with experimental materials and methods. The preparation of QDs: NF-kB p65 conjugates along with stimulation of cells for nuclear translocation of NF-kB is also discussed.

\subsubsection{Current intracellular delivery methods}

A major challenge is the efficient intracellular delivery of monodispersed QDsbioconjugates freely dispersed in the cytoplasm. Intracellular delivery methods for QDs are classified into nonspecific and targeted intracellular delivery. Several methods have been developed for the delivery of QDs into cells including both biochemical methods such as endocytosis, pinocytosis, lipids and physical methods such as electroporation and

micro/nanoinjection. ${ }^{211}$ A schematic of various methods and techniques for intracellular delivery of QDs is shown in Figure 6.2 


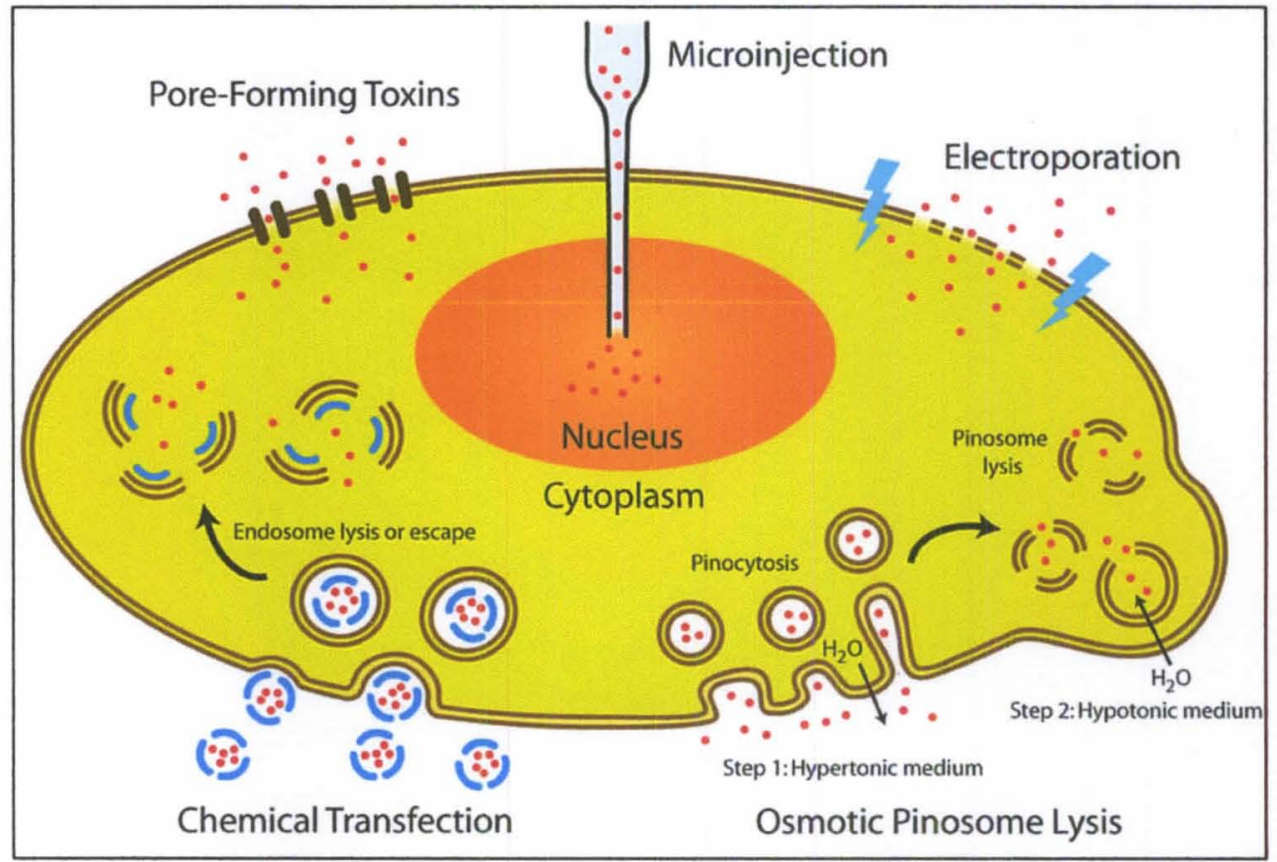

Figure 6.2 Schematic of different methods and techniques for intracellular delivery of QDs (shown in red). ${ }^{173}$

One of the most common methods for the uptake of QDs or any extracellular material, by the mammalian cells is through cell mediated methods like phagocytosis, clathrin-dependent endocytosis, macropinocytosis, caveolae-dependent endocytosis. Endocytic pathways are typically used by pathogens like viruses, bacteria and other microorganisms to gain entry into cells. Some of these pathways are also applicable for the uptake of QDs by the cells. Phagocytosis is less relevant in the intracellular delivery of QDs as it usually involves uptake of foreign pathogens like viruses or bacteria by specialized cells known as phagocytes. Clathrin-dependent endocytosis is most common for a large number of cells and is also well understood method of endocytosis which is mediated by the large protein clathrin. In this method, biomolecules and nanoparticles like QDs initially attach to the receptors or proteins present on the cell membrane and the 
protein (clathrin) assists in the formation of a coated pit on the inner surface of the plasma membrane of the cell. The clathrin coated pits are $\sim 150$ nanometer invaginated structures on the plasma membrane which occupy $2 \%$ of the plasma membrane surface. In doing so, it brings a small volume of extracellular fluid from outside to inside of the cell. Biju et al. investigated the use of this method for the delivery of QDs: peptide conjugates into live cells. ${ }^{212}$ Interestingly, it was observed that the intracellular delivery of QDs conjugates was reduced by $\sim 57 \%$ when the formation of clathrin coated pits was blocked. In this study, it was shown that though multiple pathways are involved in the intracellular delivery of conjugates, clathrin dependent endocytosis was the most important. Caveolae -dependent endocytosis involves the formation of non-clathrin coated pits formed from the plasma membrane which is more common for smooth muscle cells, fibroblasts and endothelial cells. In this method of endocytosis, biomolecules and nanoparticles like QDs were trapped in small flask shaped pits called caveolae, resembling like caves $\sim 50$ nanometers in size. Caveloin, a transmembrane protein assists in the formation of pits enriched in proteins, cholesterol and glycolipids. Both the clathrin-dependent and the caveolae-dependent endocytosis methods can be either non-specific or receptor mediated pathways.

Other non-specific methods of endocytosis for intracellular delivery of QDs are macropinocytosis, lipids/liposomes and polymers. Macropinocytosis, discovered by Warren Lewis in 1931 is a form of endocytosis which involves ruffling of the cell surface. ${ }^{213}$ The formation of relatively large macropinosomes provides a good route for non-selective endocytosis of macromolecules. Okada et al. demonstrated the introduction of macromolecules into mammalian using osmotic lysis of pinocytic vesicles. ${ }^{214}$ In this 
method, cells were first incubated in hypertonic culture medium containing sucrose, polyethylene glycol and the macromolecule to be loaded. This results in rapid formation of pinocytic vesicles formed of the plasma membrane due to movement of water out of the cells. In the next step, pinocytic vesicles containing the macromolecule to be delivered were burst by placing the cells briefly in hypotonic medium (medium diluted with DI water) which creates an internal osmotic pressure. This releases the contents in the vesicles filling the cytosol with the macromolecule. Courty et al. demonstrated the delivery of QDs conjugates into live cells using macropinocytosis to detect intracellular movement of single molecular motors. ${ }^{215}$

Surface charge and hydrophobicity are the two major factors in the endocytosis of QDs using cationic lipids and polymers. Cationic lipds/liposomes were also extensively used for the intracellular delivery of QDs due to their underlying advantages such as wide availability, biocompatibility and cost effectiveness. Derfus et al. demonstrated that the QDs complexed within transfection agents such as cationic liposomes derived from lipofectamine 2000 were endocytosed by the cells. ${ }^{216}$ QDs coated with polymers were shown to undergo nonspecific endocytosis. ${ }^{217,} 218$ Pore forming bacterial toxins like streptolysin $\mathrm{O}$ which temporarily permeabilizes the plasma membrane were also investigated for the delivery of QDs. ${ }^{173}$ However, the activity of streptolysis $\mathrm{O}$ wass not stable and was difficult to control.

The above methods though successful in the QDs delivery into the cellular cytoplasm, the QDs delivered were aggregates, limiting their utility. ${ }^{159,219}$ Endocytosis delivered QDs were trapped in the vesicles preventing the labeling of other intracellular components such as mitochondria or nucleus. Delivery of QDs as aggregates restricts 
their subsequent trafficking such as translocation to nucleus. Moreover, the biomolecule or antibody (to be delivered) attached to the QDs will remain trapped in the vesicles making it unavailable for subsequent molecular recognition. Aggregation of QDs can affect their stability as well as may result in the quenching of the fluorescence emission. More recently, chemical transfection methods like endosome-disrupting (endosomolytic) surface coatings were employed for intracellular delivery of QDs. ${ }^{220}$ The QDs were coated with copolymer ligands such as polyethylene glycol grafted polyethylenimine (PEI-g-PEG) and were rapidly internalized by the cells and released into the cytoplasm by slow endosomal escape. The presences of amine groups on each PEI molecule not only help in rapid endocytosis of across cell membranes but also in disruption of intracellular organelles though proton sponge effect. Kim et al. encapsulated antibody coated QDs within biodegradable polymeric nanospheres and the intracellular delivery was based on the hydrolysis of the polymeric spheres once inside the cells. ${ }^{221}$ Though these methods help in the delivery of QDs into the cytosol; the delivery is not homogenous. Moreover, the mechanism of the release of the QDs from the endosomes is not well understood. Peptide conjugated QDs were also investigated for the efficient intracellular delivery. The most commonly used peptide is Tat peptide (trans-activating transcriptional activator) which was readily uptaken by many cell types due to its net positive charge. Ruan et al. demonstrated that the QDs conjugated with Tat peptides attach to the negatively charged cell membranes through electrostatic interactions and internalized through macropinocytosis. ${ }^{222}$ 
Physical methods like electroporation were also utilized to deliver large quantities of QDs into cells. ${ }^{216}$ An electric pulse charges the cell membranes resulting in the rearrangement of lipid molecules. This creates small hydrophilic pores in the cell membrane through which foreign particles and extracellular fluid can enter the cytoplasm. Even though large numbers of QDs were delivered using this technique, the delivery is not homogenous and the electrical pulses may result in high cell mortality. ${ }^{223}$ The only technique that has been shown to accomplish homogenous distribution of QDs into the cytosol or nucleus without endosomal trapping is microinjection. ${ }^{216,166}$ In this technique, a small amount (typically few femtoliters) of QDs were introduced into the cells using a very fine capillary or a needle using a fluorescent microscope to guide the targeting of the cells. Dubertret et al. microinjected CdSe/ZnS QDs encapsulated in phospholipid micelles into Xenopus embryonic cells for in vivo imaging. ${ }^{166}$ Microinjection was also used for delivery of QDs for intracellular labeling of specific organelles such as selective labeling of nucleus and mitochondria ${ }^{216}$ Chen et al. developed a nanoinjector made of carbon nanotubes and demonstrated delivery of protein conjugated QDs into live human cells. ${ }^{224}$ The major disadvantages of microinjection technique are that it is highly operator dependent, laborious and low throughput process accomplished by injecting one or few cells at a time. ${ }^{216,166}$ The efficiency of microinjection is also dependent on physical constraints like cell morphology, membrane thickness and cell height. Further, the equipment needed to perform microinjection is usually very expensive relative to other techniques. Therefore, a more efficient method to accomplish freely diffused QDs inside live cells is needed for labeling of cellular components as well as intracellular delivery of biomolecules. 


\subsubsection{Reversible permeabilization of live cells}

A major barrier for the entry of extracellular molecules into cell interior is the plasma membrane. Selective and reversible permeabilization of the plasma membrane is necessary for intracellular delivery of biomolecules and nanoparticles. Permeability of the plasma membrane to various biomolecules is related to the structure of the hydrophilic lipid bilayer exposed to the aqueous environment and the fatty acyl chains that form the hydrophobic core. Permeabilization of plasma membrane involves the formation of transient defects and passage of the permeant molecules across the membrane through these defects. Permeability is also dependent on the physical properties, composition and the homogeneity of the membrane as well as the polarity and size of the permeant molecule. For successful intracellular delivery, permeabilization method should satisfy two important criteria. Firstly, it should be selective to the specific target membrane without compromising other areas of the cell. For example, some applications require permeabilization of only plasma membrane without affecting other intracellular organelles like mitochondria or nucleus. Secondly and most importantly, the permeabilization should be reversible especially for applications which require long term viability of the cells. ${ }^{225}$

Chemical detergents like Triton X-100, Saponin and digitonin are the commonly used cell permeabilization agents for intracellular delivery of biomolecules like antibodies and fluorescent proteins. However, they were largely used in immunohistochemistry applications involving fixed/dead cells and tissues. Triton-X was successfully used to reversibly permeabilize live cells for delivery of optical contrast agents ranging from $1 \mathrm{kDa}$ to $150 \mathrm{kDa}$ in molecular weight. ${ }^{226}$ It was reported that 
treatment of cells with near minimum concentrations of Triton-X, membrane permeabilization was reversible and its integrity was typically restored after 24 hours. However, Triton X-100 has relatively lower selectivity in applications which require selective permeabilization of cytoplasm leaving the nucleus intact. It was also reported that use of Triton X-100 may cause loss of detection of some membrane proteins. ${ }^{227} \mathrm{~A}$ non-ionic detergent, digitonin was used to induce reversible permeabilization of plasma membrane in cells. ${ }^{228}$ Treatment of cells with low concentrations of digitonin was shown to selectively permeabilize the plasma membrane due to its high cholesterol content. The nuclear envelope was left intact and the cytoskeleton was largely undisturbed. ${ }^{229,230}$ The mechanism in which the membrane reseals following the treatment with digitonin is not well understood. However, it was largely attributed to the presence of $\mathrm{Ca}^{+2} \cdot{ }^{231}$ Lowe et al. investigated the selectivity of nuclear pore complex in digitonin pemeabilized cells using protein conjugated QDs. ${ }^{232}$

Saponin, another cell permeabilization agent, is a plant derived glycoside known for its foaming and hemolytic activity. ${ }^{233}$ The membrane solubilizing effect of Saponin is mainly attributed to its ability to form complexes with cholesterol. A mixture of Saponin and cholesterol results in the formation hexagonal rings of diameters $\sim 8$ nanometers with the Saponin carbohydrate domains at the center without any membrane discontinuities. ${ }^{234}$, ${ }^{235}$ Scanning electron microscopy revealed that Saponin creates holes in the cell surface membrane in the size ranges from 100 nanometers to 1 micrometer. ${ }^{236}$ Wassler el al. demonstrated the use of different concentrations of Saponin to accomplish differential permeabilization of membranes. ${ }^{237}$ Saponin was also used for permeabilization of cells as well as for the introduction of macromolecules like trypan blue and exogenous tRNA. ${ }^{238}$ 
It was reported that when cells were treated with Saponin, there was minimal release of the intracellular macromolecules with minimal damage to the internal architecture and the protein synthesis remained at comparable levels with that of intact cells. ${ }^{239}$ Moreover, treated cells allowed the entry of molecules of at least $800 \mathrm{kDa}$. Johnson et al. demonstrated reversible permeabilization of neonatal cardiac myocytes using Saponin and accomplished the delivery of peptides into cells. ${ }^{240}$

In this dissertation, a new technique for homogenous and endocytosis free delivery of QDs using reversible permeabilization of live cells will be presented. This is acheved using low concentrations of Saponin in the presence of hypotonic environment. To minimize the effect of endocytic internalization pathway of QDs, experiments were performed at $4^{\circ} \mathrm{C}$ to minimize the energy available for endocytosis. ${ }^{241}$ Saponin permeabilization however allows bidirectional transport (i.e.) into and out of the cell which can compromise cellular processes via loss of vital intracellular molecular contents. To ensure unidirectional transport into the cell, Saponin is used in combination with hypotonic exposure to enhance osmosis driven transport of QDs into the cell while minimizing leakage of intracellular contents out of the cell. Hypotonic exposure causes an influx of fluid into the cell due to the osmotic pressure gradient which can be exploited to transport QDs along with the fluid into the cytoplasm of the cell. Several studies have demonstrated that the osmosis based methods can be used to transport fluids ${ }^{242,243,244}$ and other macromolecules into cells. ${ }^{214,} 245,246$ Saponin in combination with hypotonic conditions was found to be extremely efficient in accomplishing endocytosis-free delivery of QDs into the cellular cytoplasm while maintaining cell viability. Further, the 
QDs delivered were vesicle-free and were well dispersed within the cell for imaging and biomolecular delivery applications.

\subsection{EXPERIMENTAL MATERIALS AND METHODS}

\subsubsection{Materials}

Water soluble CdSe/ZnS core/shell QDs with emission $~ 530 \mathrm{~nm}$, hydrodynamic size of $26 \pm 6.07$ nanometers and zeta potential of $-43.05 \mathrm{mV}$ are used which are purchased from Ocean Nanotech, LLC (Springdale, AR). The NF-kB p65 antibodies (SC-8008) without gelatin are obtained from Santa Cruz biotechnology (Santa Cruz, CA). Custom conjugation of QDs (em 530) with NF-kB p65 antibodies is obtained from Ocean Nanotech, LLC and is also performed using Qdot 605 antibody conjugation kit (Invitrogen). Saponin for reversible permeabilization experiments is purchased from Alfa Aesar (Ward Hill, MA) and deionized water (DI) for the hypotonic experiments is obtained for Milli-Q filtration system purchased from Millipore (Billerica, MA). Cell

stimulation is performed using bacterial LPS (Sigma Aldrich, St. Louis, MO), TNF- $\alpha$ (BD biosciences, San Jose, CA) and UV lamp (in laboratory cell culture hood). Paraformaldehyde used for fixing of the cells, Triton X-100 used for permeabilization, BSA (bovine serum albumin) used to block non-specific binding are obtained from Sigma Aldrich(St. Louis, MO). All other reagents like PBS, Tween-20,sucrose, potassium chloride, potassium acetate and HEPES are obtained from Sigma Aldrich. (St. Louis, MO)

\subsubsection{Cell culture}

All experiments utilized a rat cardiomyocyte cell line; H9c2 cells (American Type Culture Collection). The H9c2 cells are cultured in Dulbecco's Modification of Eagle's 
Medium (DMEM) with $4.5 \mathrm{~g} / \mathrm{L}$ glucose, L-glutamine, and sodium pyruvate (Fischer Scientific), $10 \%$ fetal bovine serum (FBS, Hyclone) and $1 \%$ antibiotics (penicillin and streptomycin, Mediatech). Cells ae cultured in LabTek chambered cover glass (Fischer Scientific) and in multi-well plates (BD falcon) at a density of approximately $1.2 \times 10^{4}$ cells $/ \mathrm{cm}^{2}$. Trypsin EDTA (Mediatech) is used to detach the cells from the surface for flow cytometry (BD FACS calibur) studies.

\subsubsection{Blocking of endocytosis at $4^{\circ} \mathrm{C}$}

Mammalian cells when exposed to QDs at room temperature readily internalize QDs through energy dependent endocytosis. ${ }^{219} \mathrm{H} 9 \mathrm{c} 2$ cells are used as representative mammalian cell line to evaluate their response to QDs delivered at both room temperature and $4^{\circ} \mathrm{C}$. Cells seeded are treated with QDs ( 10nM) for 10 minutes at room temperature as well as at $4^{\circ} \mathrm{C}$ in serum free media. Cells alone without QDs are used as negative control. After the incubation, cells are washed with 1x PBS and media and observed under a confocal microscope (Nikon A1 confocal system). In order to evaluate the efficacy of the reversible permeabilization technique as well as ensure endocytosisfree intracellular delivery of QDs, all the experiments were performed at $4^{\circ} \mathrm{C}$.

\subsubsection{Reversible permeabilization using hypotonic buffer}

H9c2 cells are seeded at a density of approximately $1.2 \times 10^{4} \mathrm{cells} / \mathrm{cm}^{2}$. After 24 hours, the media is removed and washed twice with $1 \mathrm{x}$ PBS for cell internalization studies. Hypotonic buffer solutions at three different ratios $(1: 1,3: 2,1: 3)$ are prepared by mixing $\mathrm{S}$ buffer $^{239}(130 \mathrm{mM}$ sucrose, $50 \mathrm{mM} \mathrm{KCl}, 50 \mathrm{mM}$ potassium acetate, $20 \mathrm{mM}$ HEPES, pH 7.4) with DI water at respective ratios. Cells are treated with QDs ( 10nM) mixed in the three hypotonic solutions at a pH around 7.4 for 2 minutes, 5 minutes and 10 
minutes at $4^{\circ} \mathrm{C}$ on a rotary shaker. After each time point, the cells are washed twice with the respective hypotonic buffer solutions, once with $1 \mathrm{x}$ PBS and thoroughly washed with regular media. Cells are then incubated at $37^{\circ} \mathrm{C}$ for $30-45 \mathrm{~min}$ for recovery before imaging using confocal microscopy.

\subsubsection{Reversible permeabilization using Saponin}

Saponin solutions are prepared at three different concentrations $25 \mu \mathrm{g} / \mathrm{ml}, 50 \mu \mathrm{g} / \mathrm{ml}$ and $75 \mu \mathrm{g} / \mathrm{ml}$ in hypotonic buffer (3:2 ratio of buffer to DI water). Cells are treated with QDs ( 10nM) mixed with the above hypotonic Saponin solutions for 2 minutes, 5 minutes and 10 minutes at $4^{\circ} \mathrm{C}$ on a rotary shaker. After each time point, the cells are washed twice with hypotonic $S$ buffer (3:2 ratio) solution, once with $1 \times$ PBS and thoroughly washed with regular media and incubated for $30-45$ minutes at $37^{\circ} \mathrm{C}$ for recovery. Control experiments are done by treating cells with and without QDs $(\sim 10 \mathrm{nM})$ in $1 \mathrm{x}$ PBS for 10 minutes and without Saponin or hypotonic conditions. Cell permeabilization and internalization of QDs is confirmed by imaging live cells using confocal microscopy.

\subsubsection{Evaluation of cell viability}

Following hypotonic and Saponin treatments, reversible permeabilization is confirmed by evaluating cell viability using the live/dead viability/cytotoxicity kit (Invitrogen). Cells are cultured in multi-well plate and the viability study is done following the instructions in the kit. Briefly, $6 \mu$ l of Calcein AM and $15 \mu$ l of Ethidium homodimer are mixed well in $12 \mathrm{ml}$ of $1 \mathrm{x}$ PBS for 15 minutes. After the recovery of the cells for 30-45 minutes following hypotonic and Saponin treatments, cells are treated with $400 \mu$ l of the prepared calcein-ethidium homodimer solution and incubated at $37^{\circ} \mathrm{C}$ 
for 30-45 minutes. Positive and negative control experiments are cells treated with Saponin without hypotonic conditions and cells without Saponin or hypotonic buffer respectively. The cells are then carefully washed with $1 x$ PBS and imaged under a fluorescent microscope (Nikon eclipse TE 2000-U). Live cells are distinguished by the presence of ubiquitous intracellular esterase activity, determined by the enzymatic conversion of the virtually non-fluorescent cell-permeant calcein AM to the intensely fluorescent calcein. The polyanionic dye calcein is well retained within live cells and produces an intense uniform green fluorescence (ex/em $\sim 495 \mathrm{~nm} / \sim 515 \mathrm{~nm}$ ) in live cells. EthD-1 enters cells with damaged membranes and undergoes a 40-fold enhancement of fluorescence upon binding to nucleic acids, thereby producing a bright red fluorescence (ex/em $\sim 495 \mathrm{~nm} / \sim 635 \mathrm{~nm})$ in dead cells. EthD-1 is excluded by the intact plasma membrane of live cells. The cell viability assay is performed without the QDs due to the overlapping of their emission with the calcein dye used.

\subsubsection{Significance of hypotonic buffer conditions}

The significance of hypotonic buffer environment is evaluated by treating H9c2 cells with Saponin in the absence of the hypotonic buffer conditions. The chosen concentration of Saponin for this study is $125 \mu \mathrm{g} / \mathrm{ml}$. This concentration is higher than the concentrations used for the study with hypotonic buffer $(25-75 \mu \mathrm{g} / \mathrm{ml})$, however, in the absence of hypotonic buffer the lower concentrations of Saponin resulted in poor intracellular QD delivery. The $125 \mu \mathrm{g} / \mathrm{ml}$ is the lowest concentration of Saponin at which appreciable uptake of QDs in the cells is observed. Cells treated with $1 \mathrm{mg} / \mathrm{ml}$ of and 0 $\mathrm{mg} / \mathrm{ml}$ of Saponin for 10 minutes are used as positive and negative controls respectively. 
Following the treatment, cells are recovered at $37^{\circ} \mathrm{C}$ for $30-45$ minutes and cell viability is evaluated using live/dead assay.

\subsubsection{Quantitative and qualitative analysis using fluorescence microscopy}

All images for the hypotonic and Saponin experiments are obtained using Nikon Al confocal fluorescent microscope using of $60 \mathrm{x}$ magnification. The brightness and contrast are equally readjusted for all the images in Adobe Photoshop. All the images for the cell viability assay are obtained using Nikon eclipse TE 2000-U fluorescent microscope. The percentage of live and dead cells are obtained from the fluorescent images by counting the number of cells showing green (live) and red (dead) fluorescence using metamorph software.

\subsubsection{Quantitative analysis using flow cytometry}

The mean fluorescence intensity (MFI) or the G mean of the cell internalized QDs

is assessed using flow cytometry. Following the hypotonic and Saponin treatments of cells with QDs, cells are detached from the plate using Trypsin-EDTA and suspended in flow buffer for flow cytometry. Cells are visualized by the strong green $(\sim 530 \mathrm{~nm})$ fluorescence from the QDs using the signal from the FL1 band pass emission $(530 \pm 30)$ filter. The population histograms showing the fluorescence intensity of QDs from the cells is generated using Cell Quest Pro software.

\subsection{ODS: NF-KB P65 ANTIBODY CONJUGATES}

QDs are conjugated with NF-kB p65 antibodies using standard conjugation methods. Carboxyl terminated QDs (em $\sim 530 \mathrm{~nm}$ and $\sim 600 \mathrm{~nm})$ are activated using EDAC and then either directly conjugated to the amino groups present on the NF-kB p65 antibodies or via a fusion protein, protein $\mathrm{G}(\mathrm{pG})$. Custom conjugation of NF-kB p65 
antibodies with QDs is obtained from Ocean Nanotech LLC. The conjugation of QDs with the antibodies is confirmed using gel electrophoresis. QDs (em $\sim 605 \mathrm{~nm}$ ) are also conjugated with NF-kB p65 antibodies using commercially available conjugation kit (Invitrogen). Briefly, the QDs are amine derivatized and coated with PEG groups. The conjugation of the QDs with the NF-kB p65 antibodies is performed by first activating the QDs using SMCC to generate reactive maleimides groups. Following this step, the activated QDs are coupled to the antibodies by coupling the reactive maleimides groups to the thiol groups present in the NF-kB p65 antibodies. In this dissertation, QDs with emission $\sim 530 \mathrm{~nm}$ are used for intracellular delivery study while QDs with emission $530 \mathrm{~nm}$ and $\sim 600 \mathrm{~nm}$ are used for labeling of NF-kB protein study.

\subsubsection{Stimulation of cells for activation of NF-kB}

H9c2 cells are cultured in regular media LabTek chambered cover glass at a density of approximately $1.2 \times 10^{4}$ cells $/ \mathrm{cm}^{2}$. The seeded cells are starved in media without serum and antibiotics overnight prior to stimulation. Cells are then stimulated for the activation and nuclear translocation of NF-kB with LPS, TNF- $\alpha$ and UV radiation. Serum starved cells are stimulated with LPS at a concentration of $\sim 100 \mu \mathrm{g} / \mathrm{ml}$ for 2 hours and with TNF- $\alpha$ at a concentration of $\sim 50 \mathrm{ng} / \mathrm{ml}$ for 1 hour to at $37^{\circ} \mathrm{C}$. Cells are stimulated with UV radiation using the standard UV lamp in cell culture hood for 2 hours at room temperature.

\subsubsection{Immunofluorescence microscopy}

Following the cells stimulation, the activation and nuclear translocation of NF-kB, is detected using the QDs fluorescence by performing standard immunofluorescence microscopy. Briefly, after the stimulation, cells are fixed using $2 \%$ paraformaldehyde for 
20 minutes at $37^{\circ} \mathrm{C}$. Cells are washed with $1 \times$ PBS twice and permeabilized using $0.1 \%$ Triton X-100 for 5 minutes at room temperature. After the permeabilization, cells are washed twice with wash buffer $(0.05 \%$ Tween-20 in $1 \mathrm{x}$ PBS). Non-specific binding is blocked by incubating the cells with $0.5-1 \%$ BSA in $1 \times$ PBS for 30 minutes at room temperature. QDs: NF-kB p65 conjugates ( $10 \mathrm{nM}$ concentration) are added to the cells and allowed to incubate for 1 hour at room temperature on a rotary shaker. Following the incubation with the QDs conjugated antibodies; cells are thoroughly washed three times with wash buffer for 5 minutes. The successful labeling the NF-kB protein using the QDs: NF-kB p65 conjugates is confirmed by imaging the cells using confocal microscopy. 


\section{CHAPTER VII -RESULTS AND DISCUSSION}

This chapter deals with the results obtained in the intracellular delivery of QDs using the reversible permeabilization method. The discussion section deals with interpretation of the observed results. The preliminary results showing the conjugation of QDs with NF-kB p65 antibodies and ability of QDs to image the nuclear translocation of NF-kB upon cell stimulation are presented at the end of the chapter.

\subsection{RESULTS}

This section deals with the results from the reversible permeabilization experiments for the intracellular delivery of QDs.

\section{1.1 Endocytosis blocked at $4^{\circ} \mathrm{C}$}

Several studies have indicated that mammalian cells exposed to nanomaterials like carbon nanotubes and QDs at room temperature readily internalized them via endocytic pathways whereas exposure at $4^{\circ} \mathrm{C}$ inhibited endocytosis. ${ }^{247,219}$ Using $\mathrm{H} 9 \mathrm{c} 2$ cells as a representative mammalian cell line, the uptake of QDs delivered at both room temperature and $4^{\circ} \mathrm{C}$ is evaluated. It is observed that the QDs are internalized by the cells when they are incubated at room temperature via energy dependent endocytic pathway for the uptake of QDs. The uptaken QDs are seen as aggregates as evident from the punctuate fluorescence typically seen in endocytic uptake mechanism. 
(Figure 7.1). However, minimal uptake is observed when the cells are incubated with QDs at $4^{\circ} \mathrm{C}$ (Figure 7.2). This shows that the endocytic pathways can be blocked by incubating the QDs at low temperature. Minimal or low auto fluorescence is observed in the cells incubated without the QDs (negative control) as shown in Figure 7.3.

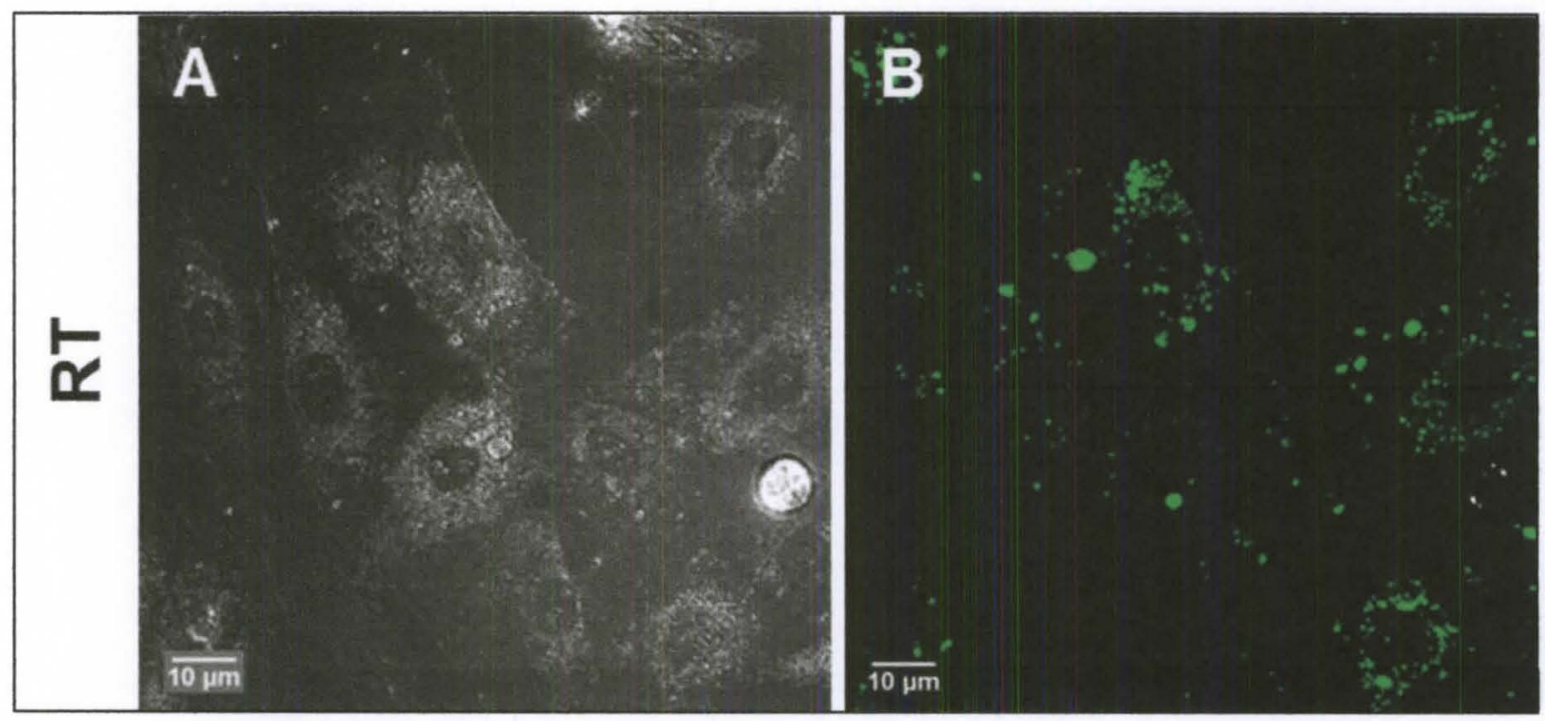

Figure 7.1. Confocal image of H9c2 cells showing endocytosis like uptake of QDs (A) Phase contrast, (B) Fluorescence images of cells incubated at room temperature (RT) with QDs. The punctuate fluorescence is indicative of endocytic uptake pathway. The images are taken with 60x magnification.

In order to test the efficiency of the reversible permeabilization method and to ensure endocytosis-free delivery, all the QDs internalization experiments are performed at $4^{\circ} \mathrm{C}$. Therefore, the results obtained can be interpreted based only on the reversible permeabilization mechanism eliminating the endocytic pathways. 


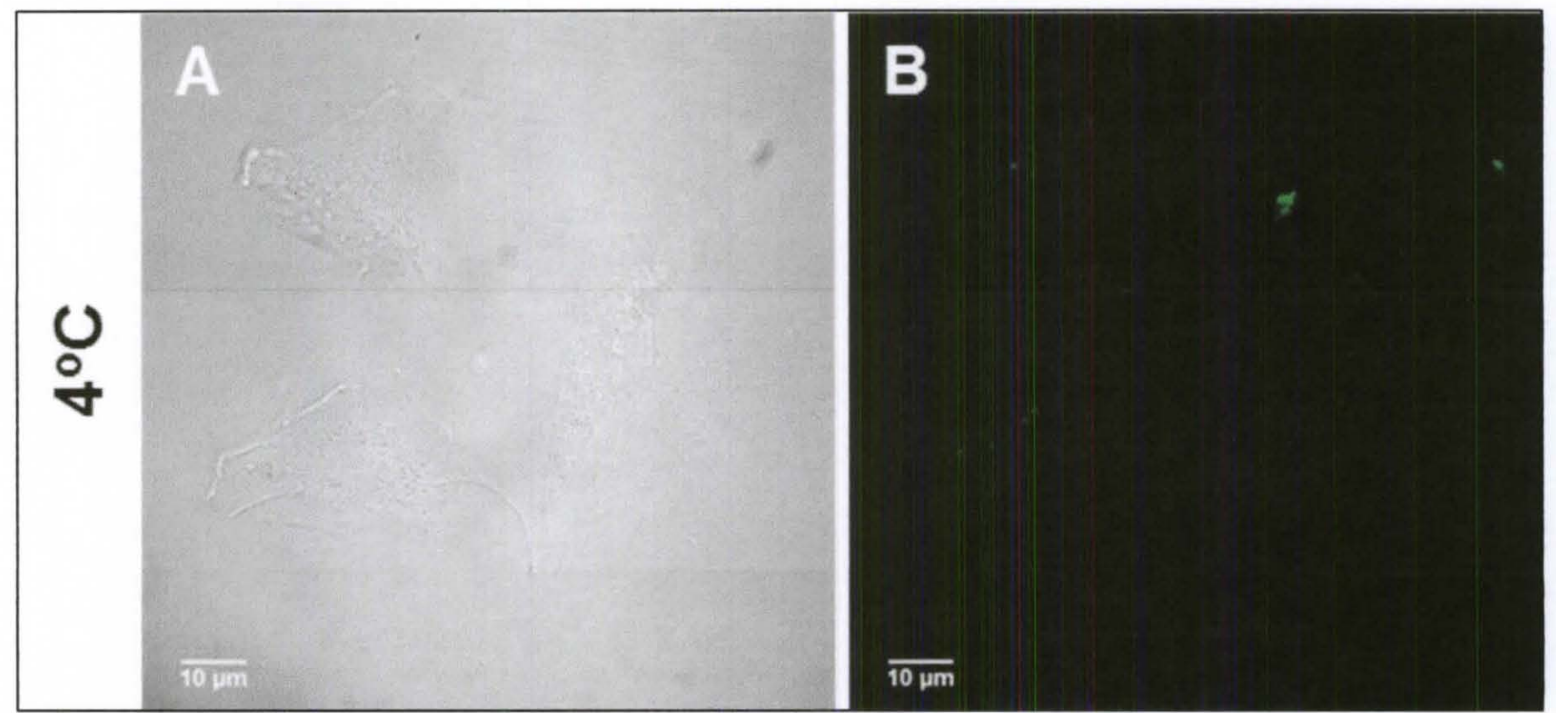

Figure 7.2. Confocal image of H9c2 cells showing blocking of endocytosis of QDs (A) Phase contrast, (B) Fluorescence images of cells incubated at $4^{\circ} \mathrm{C}$ with QDs. Minimal or very low fluorescence is observed indicating the successful blocking of endocytosis. The images are taken with 60x magnification.

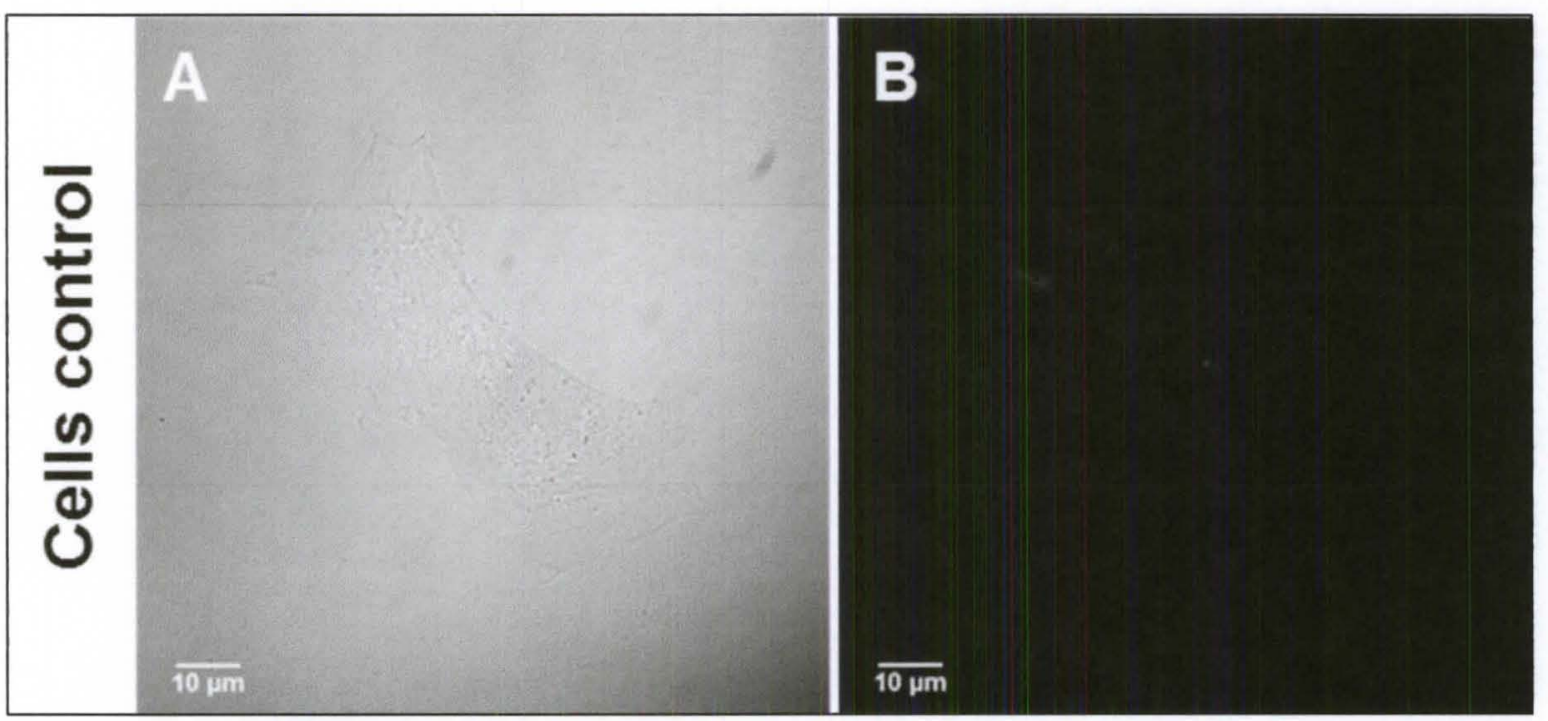

Figure 7.3. Confocal image of H9c2 cells without QDs as negative control (A) Phase contrast, (B) Fluorescence images of cells incubated without QDs. Minimal or very low auto fluorescence from the cells is observed. 


\subsubsection{Reversible permeabilization using hypotonic buffer}

This section deals with the results obtained from the reversible permeabilization experiments for the intracellular delivery of QDs using hypotonic buffer conditions. H9c2 cells cultured for 24 hours are treated with QDs $(\sim 10 \mathrm{nM})$ mixed in different hypotonic buffer ratio conditions $(1: 1,3: 2,1: 3)$ for 2 minutes, 5 minutes and 10 minutes. As the experiments are performed at $4^{\circ} \mathrm{C}$, the uptake of QDs occurred largely due to osmotic loading method. Live cell confocal microscopy is performed to confirm the internalization and distribution of the QDs in the cells. Confocal phase contrast and fluorescence images of cells internalized QDs when treated with 1:1 (Figure 7.4), 3:2 (Figure 7.5) and 1:3 (Figure 7.6) buffer: DI water ratios for 2 minutes (A, B), 5 minutes (D, E) and 10 minutes $(\mathrm{G}, \mathrm{H})$ respectively are shown. It is observed that the QDs internalized are well distributed as seen from the fluorescence signal from the cytoplasm of the cells (Figures 7.4, 7.5 and 7.6). Furthermore, when the cells are treated with 3:2 buffer: DI water hypotonic ratio for 5 minutes (Figure 7.5 D, E), the internalized QDs are homogenously distributed in the cytoplasm as seen from the diffused fluorescence signal.

Cell viability following the hypotonic treatments is analyzed from live/dead assay. Representative cell viability fluorescence images shown in the panels C, F and I (Figures 7.4, 7.5 and 7.6) show fluorescence overlay of calcein AM (green: live cells) and ethidium homodimer-1 (red: cells with damaged cell membrane) for the three different hypotonic buffers ratios $(1: 1,3: 2,1: 3)$ for the different times ( 2 minutes, 5 minutes, 10 minutes) respectively. High number of live cells and minimal or low number of dead cells

is observed as evident from high green and minimal red fluorescence signal as a 
consequence of exposure to the different hypotonic buffer ratios. However, swelling of the cells is observed when higher hypotonic ratios and with longer incubation times.

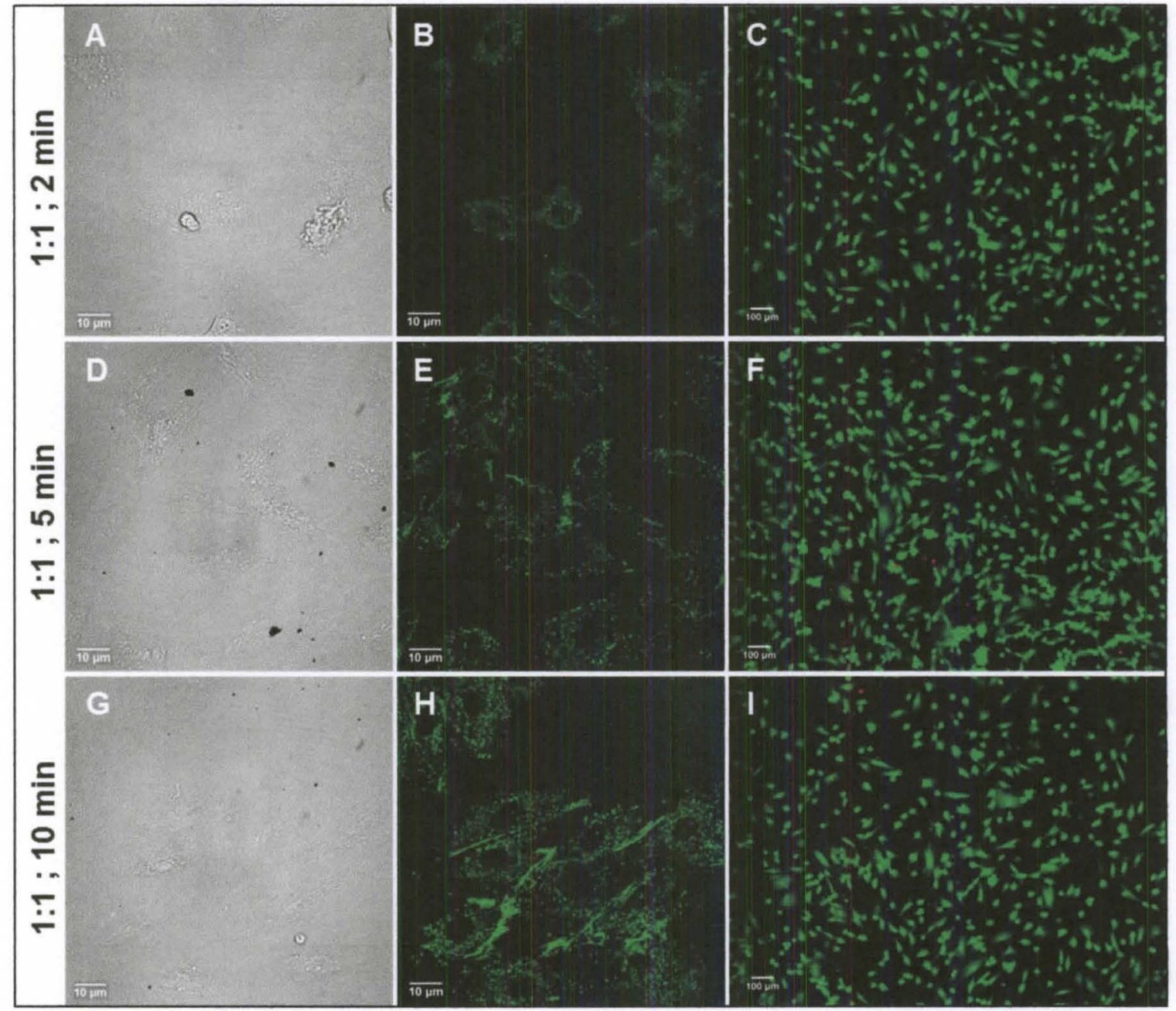

Figure 7.4. Reversible permeabilization using 1:1 hypotonic buffer ratio and cell viability. Comparison of bright field, fluorescence and viability representative images of H9c2 cells treated with 1:1 hypotonic buffer ratio for uptake of QDs for (A-C) 2 minutes, (D-F) 5 minutes and (G-I) 10 minutes respectively at $4^{\circ} \mathrm{C}$. Bright field and fluorescence images are obtained with $60 \times$ magnification and cell viability images are obtained with $10 \times$ magnification. Cell viability fluorescence images (C, F and I) show fluorescence overlay 
of calcein AM (green: live cells) and ethidium homodimer-1 (red: cells with damaged cell membrane).

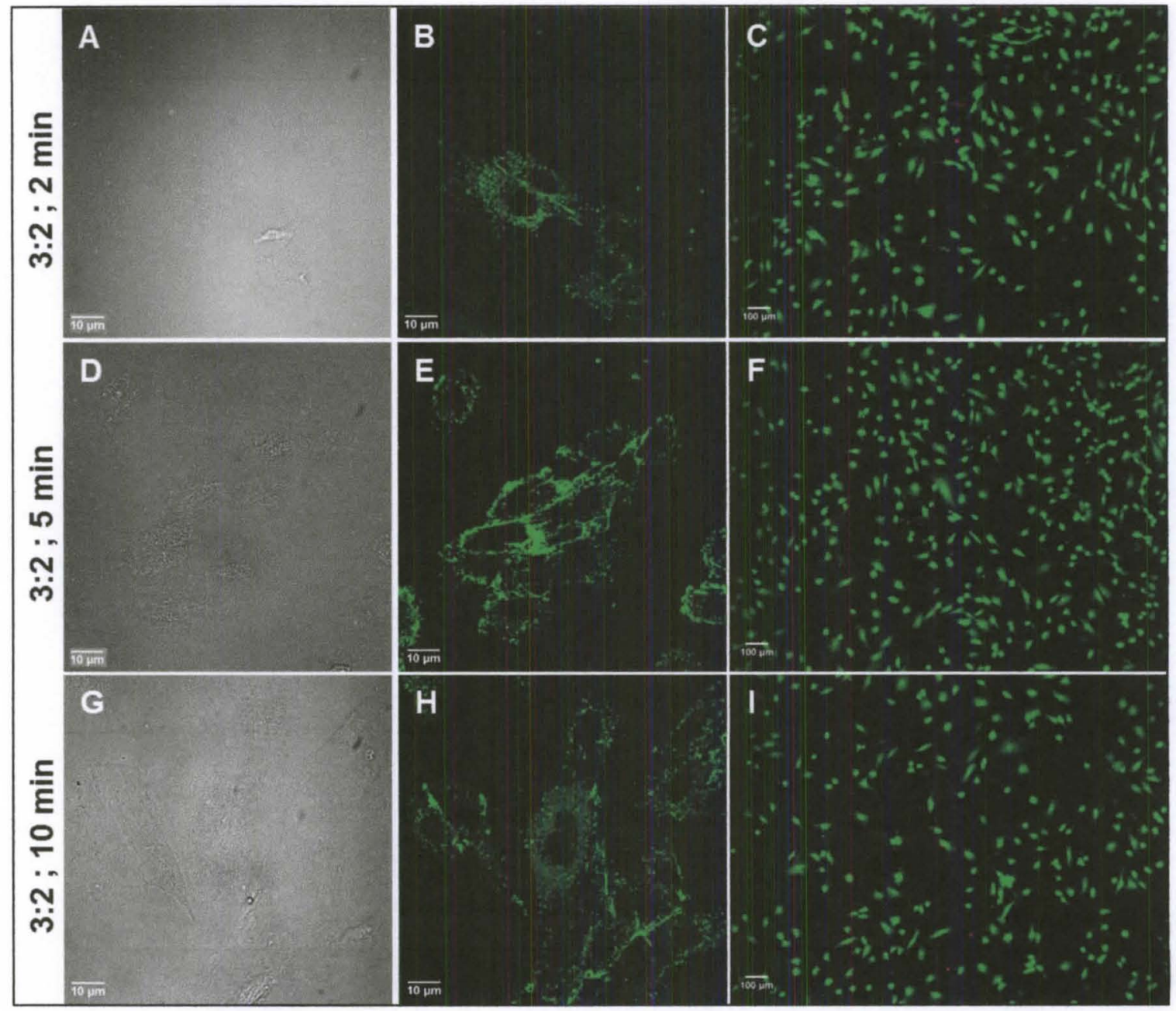

Figure 7.5 Reversible permeabilization using 3:2 hypotonic buffer ratio and cell viability. Comparison of bright field, fluorescence and viability representative images of H9c 2 cells treated with 1:1 hypotonic buffer ratio for uptake of QDs for (A-C) 2 minutes, (D-F) 5 minutes and (G-I) 10 minutes respectively at $4^{\circ} \mathrm{C}$. Bright field and fluorescence images are obtained with $60 \times$ magnification and cell viability images are obtained with $10 \times$ magnification. Cell viability fluorescence images (C, F and I) show fluorescence overlay 
of calcein AM (green: live cells) and ethidium homodimer-1 (red: cells with damaged cell membrane).

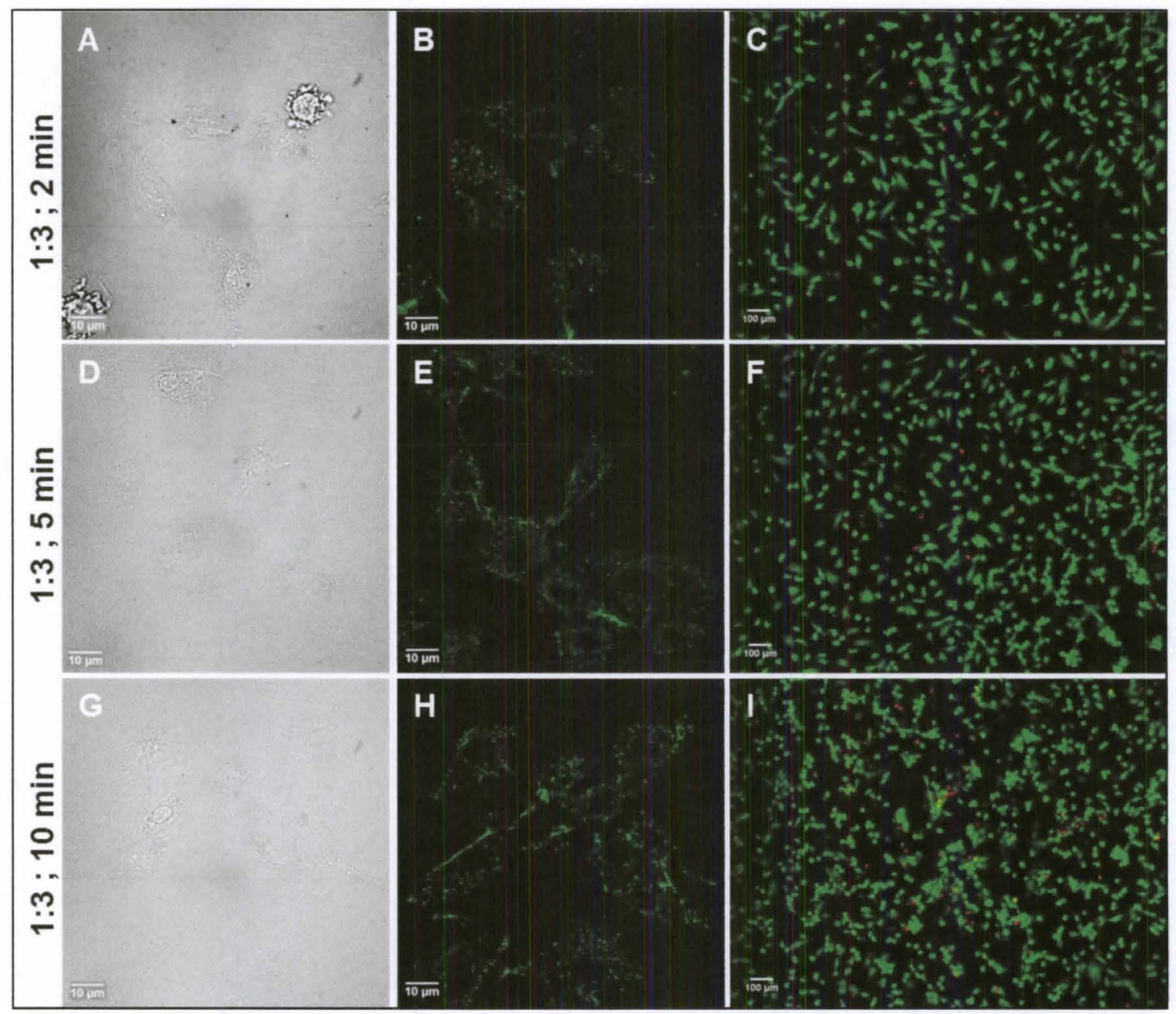

Figure 7.6. Reversible permeabilization using 1:3 hypotonic buffer ratio and cell viability. Comparison of bright field, fluorescence and viability representative images of $\mathrm{H} 9 \mathrm{c} 2$ cells treated with 1:1 hypotonic buffer ratio for uptake of QDs for (A-C) 2 minutes, (D-F) 5 minutes and (G-I) 10 minutes respectively at $4^{\circ} \mathrm{C}$. Bright field and fluorescence images are obtained with $60 \times$ magnification and cell viability images are obtained with $10 \times$ 
magnification. Cell viability fluorescence images $(\mathrm{C}, \mathrm{F}$ and $\mathrm{I})$ show fluorescence overlay of calcein AM (green: live cells) and ethidium homodimer-1 (red: cells with damaged cell membrane).

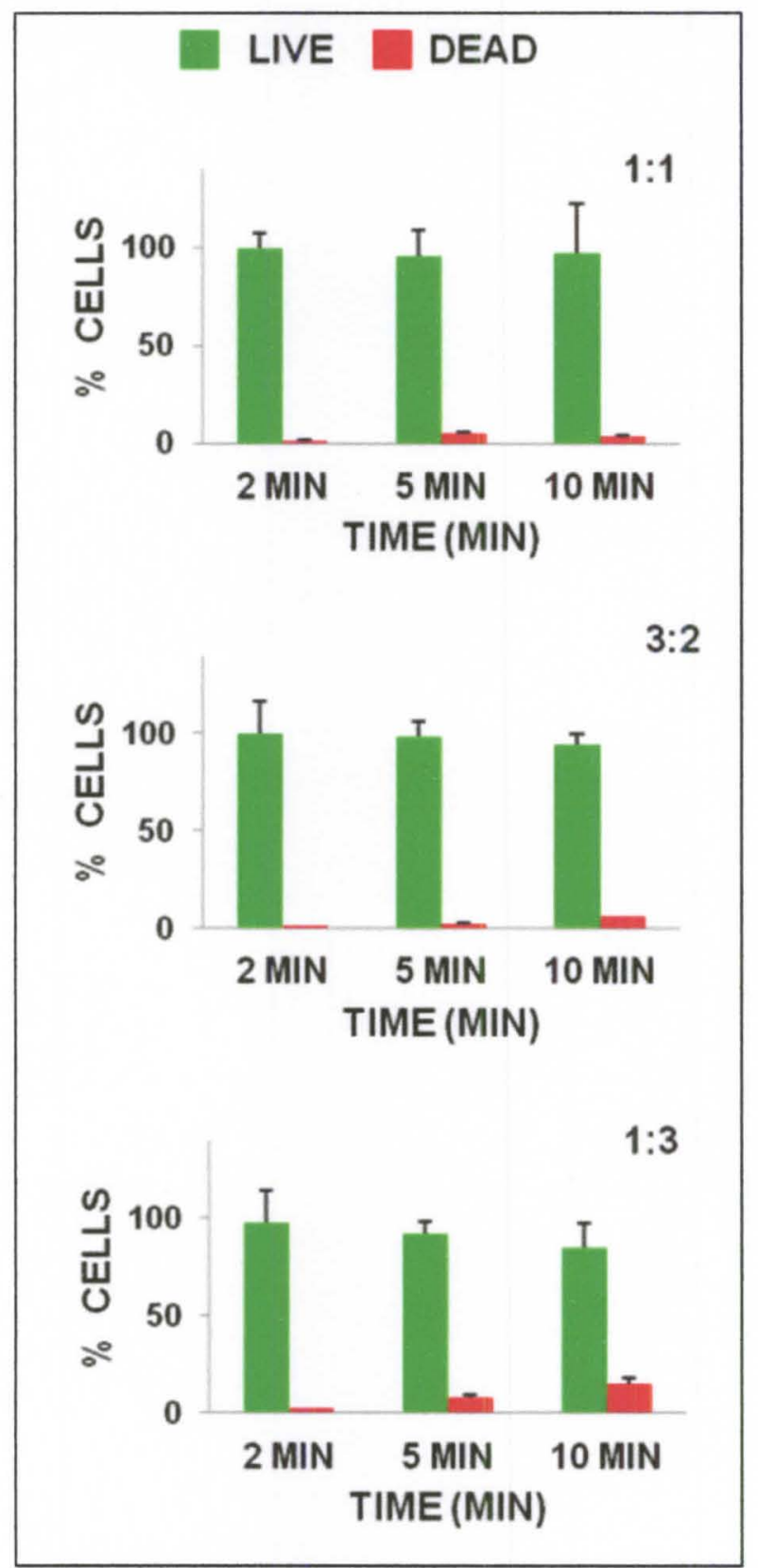

Figure 7.7 Live/Dead cell viability assay following hypotonic buffer treatment showing live cells stained with calcein AM (green: live cells) and dead cells stained with ethidium 
homodimer-1 (red: cells with damaged cell membrane). Cells are treated with different hypotonic buffer ratios $(1: 1,3: 2,1: 3)$ for different incubation times $(2,5$ and 10 minutes) at $4^{\circ} \mathrm{C}$ respectively.

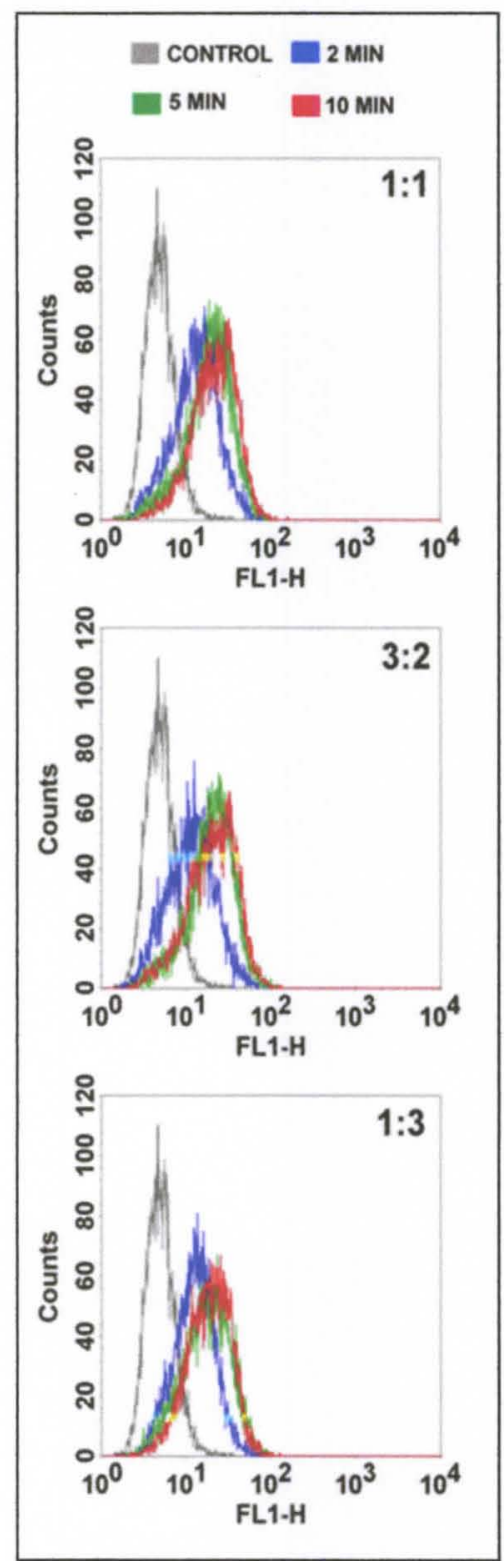

Figure 7.8 Quantitative analysis of internalized QDs with different hypotonic buffer ratios. Histograms represent mean fluorescence intensity of cells (arbitrary units) for 1:1 ratio $(12.24,17.62,20.31), 3: 2$ ratio $(10.65,18.37,18.96)$ and $1: 3$ ratio $(11.64,15.68,17.41)$ for 
2 minutes (blue), 5 minutes (green) and 10 minutes (red) respectively. The mean fluorescent intensity of cells without QDs used as negative control (gray) is 4.68.

Quantitative analysis of the percentage of live/dead cells is performed by counting the number of live and dead cells from the cell viability images for a sample size of 5 (Figure 7.7). The QDs internalized are analyzed quantitatively using flow cytometry analysis. The population histograms representing the fluorescence intensity of the cells internalized with QDs for different hypotonic buffer ratios (1:1, 3:2, 1:3) for 2 minutes, 5 minutes and 10 minutes are shown in Figure 7.8. The $G$ mean which represents the mean fluorescence intensity from the cells population for all the three different hypotonic buffer conditions is $1: 1(12.24,17.62,20.31), 3: 2(10.65,18.37,18.96)$ and $1: 3(11.64,15.68$, 17.41) for 2 minutes, 5 minutes and 10 minutes at $4{ }^{\circ} \mathrm{C}$ respectively. Cells without QDs (negative control) have a mean fluorescence intensity of 4.68 .

\subsubsection{Reversible permeabilization using Saponin}

This section deals with the results obtained from the reversible permeabilization experiments for the intracellular delivery of QDs using Saponin. H9c2 cells are treated with low concentrations of Saponin in the presence of hypotonic buffer conditions. The cells are cultured for 24 hours prior to the permeabilization experiments. The $3: 2$ hypotonic ratio is chosen for all the Saponin experiments based on the distribution of QDs inside the cells (Figure 7.5E) and the mean fluorescence intensity compared to the other ratios. Cells are treated with QDs $(\sim 10 \mathrm{nM})$ mixed in three different concentrations of Saponin: $25 \mu \mathrm{g} / \mathrm{ml}, 50 \mu \mathrm{g} / \mathrm{ml}$ and $75 \mu \mathrm{g} / \mathrm{ml}$ and are exposed for 2 minutes, 5 minutes and 10 minutes at $4^{\circ} \mathrm{C}$ in $3: 2$ hypotonic buffer ratio conditions. Live cell confocal microscopy is 
performed to evaluate the use of Saponin in conjunction with hypotonic exposure for the internalization and intracellular distribution of QDs. Confocal phase contrast and fluorescence images of cells with the internalized QDs when treated with $25 \mu \mathrm{g} / \mathrm{ml}$ (Figure 7.9), $50 \mu \mathrm{g} / \mathrm{ml}$ (Figure 7.10) and $75 \mu \mathrm{g} / \mathrm{ml}$ (Figure 7.11) in 3:2 hypotonic buffer conditions for 2 minutes $(A, B), 5$ minutes $(D, E)$ and 10 minutes $(G, H)$ respectively are shown. The results confirm that the QDs are well distributed within the cellular cytoplasm for all three conditions and show higher homogenous distribution in the cytoplasm than with hypotonic exposure alone. Particularly, the $50 \mu \mathrm{g} / \mathrm{ml}$ concentration where cells are treated for 5 minutes (Figure 7.10 D, E) shows high QD internalization and uniform distribution of QDs within the cytoplasm in comparison to the other conditions.

Representative cell viability fluorescence images shown in the panels C, F and I (Figures 7.9, 7.10 and 7.11) show fluorescence overlay of calcein AM (green: live cells) and ethidium homodimer-1 (red: cells with damaged cell membrane) from the cells treated with different hypotonic (3:2) Saponin concentrations $(25 \mu \mathrm{g} / \mathrm{ml}, 50 \mu \mathrm{g} / \mathrm{ml}$ and $75 \mu \mathrm{g} / \mathrm{ml})$ for different incubation times ( 2 minutes, 5 minutes and 10 minutes) at $4^{\circ} \mathrm{C}$ respectively. As shown in the representative cell viability images, large number of live cells and minimal or low number of dead cells is observed from high green and minimal red fluorescence signal as a consequence of exposure to the different hypotonic Saponin concentrations. However, higher Saponin concentrations or longer incubation times resulted in some cell death. Quantitative analysis of the percentage of live/dead cells is performed by counting the number of live and dead cells from the cell viability images for a sample size of 5 (Figure 7.12). The QDs internalized are analyzed quantitatively using flow cytometry analysis. The population histograms representing the fluorescence 
intensity of the cells internalized with QDs for different Saponin concentrations $(25 \mu \mathrm{g} / \mathrm{ml}$, $50 \mu \mathrm{g} / \mathrm{ml}$ and $75 \mu \mathrm{g} / \mathrm{ml}$ ) for 2 minutes, 5 minutes and 10 minutes are shown in Figure 7.13.

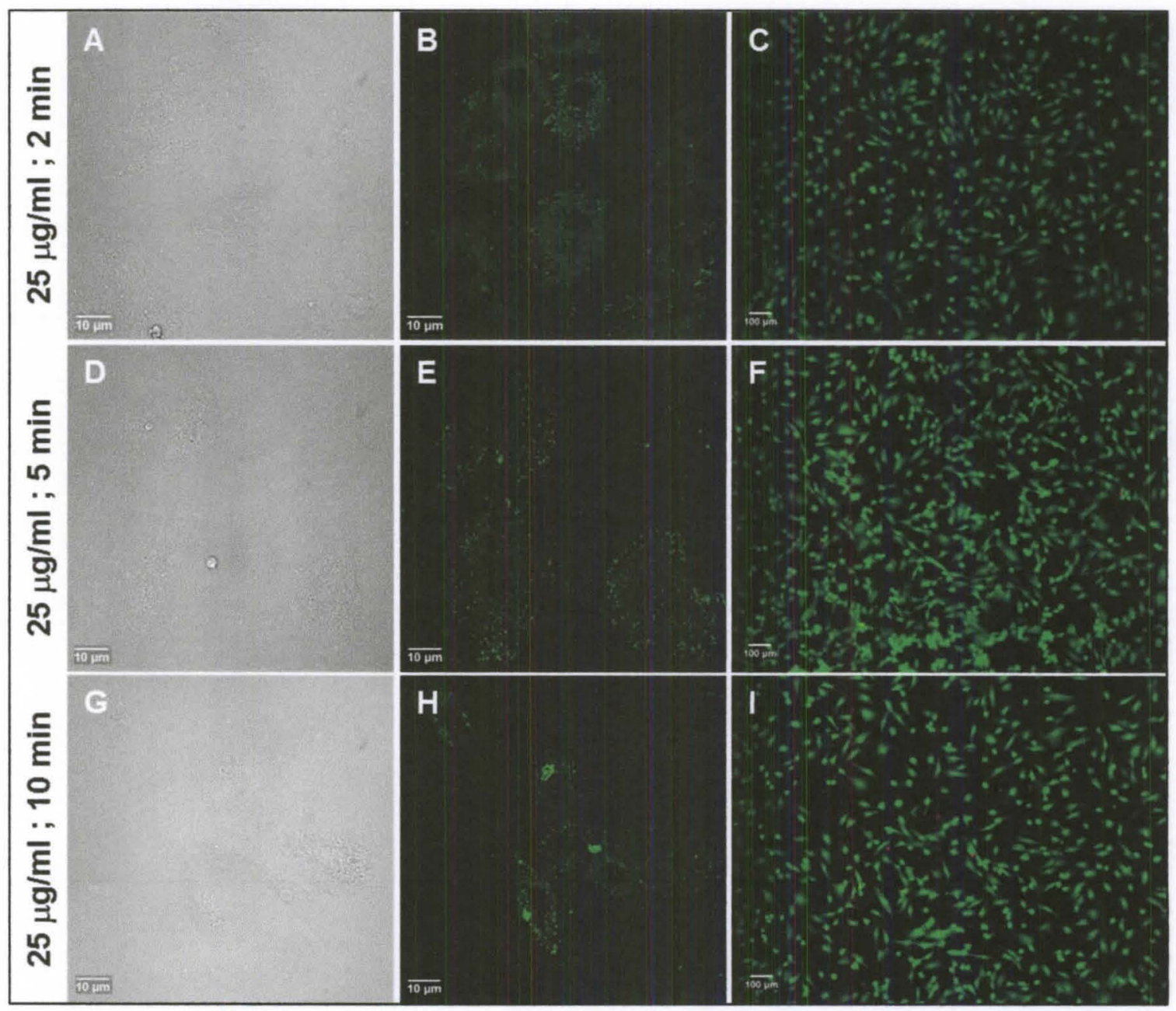

Figure 7.9 Reversible permeabilization using $25 \mu \mathrm{g} / \mathrm{ml}$ Saponin and cell viability. Comparison of bright field, fluorescence and viability representative images of H9C2 cells treated with $25 \mu \mathrm{g} / \mathrm{ml}$ of Saponin for (A-C) 2 minutes, (D-F) 5 minutes and (G-I) 10 minutes respectively. Bright field and fluorescence images are obtained with $60 \times$ magnification and cell viability images were obtained with $10 \times$ magnification. Cell viability fluorescence images $(\mathrm{C}, \mathrm{F}$ and $\mathrm{I})$ show fluorescence overlay of calcium $\mathrm{AM}$ (green: live cells) and ethidium homodimer-1 (red: cells with damaged cell membrane). 


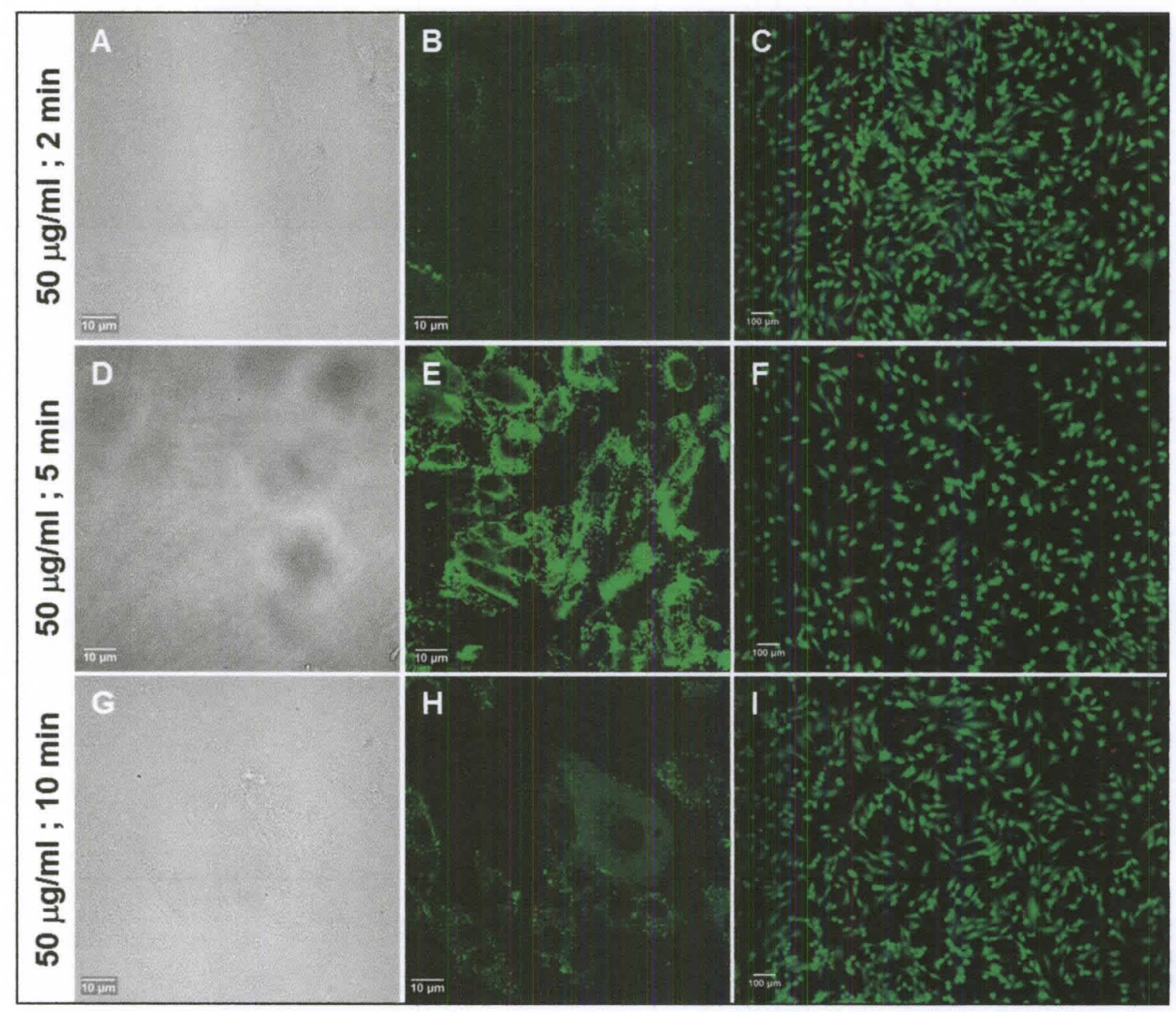

Figure 7.10 Reversible permeabilization using $50 \mu \mathrm{g} / \mathrm{ml}$ Saponin and cell viability. Comparison of bright field, fluorescence and viability representative images of $\mathrm{H} 9 \mathrm{C} 2$ cells treated with $25 \mu \mathrm{g} / \mathrm{ml}$ of Saponin for (A-C) 2 minutes, (D-F) 5 minutes and (G-I) 10 minutes respectively. Bright field and fluorescence images are obtained with $60 \times$ magnification and cell viability images were obtained with $10 \times$ magnification. Cell viability fluorescence images (C, F and I) show fluorescence overlay of calcein AM (green: live cells) and ethidium homodimer-1 (red: cells with damaged cell membrane). 


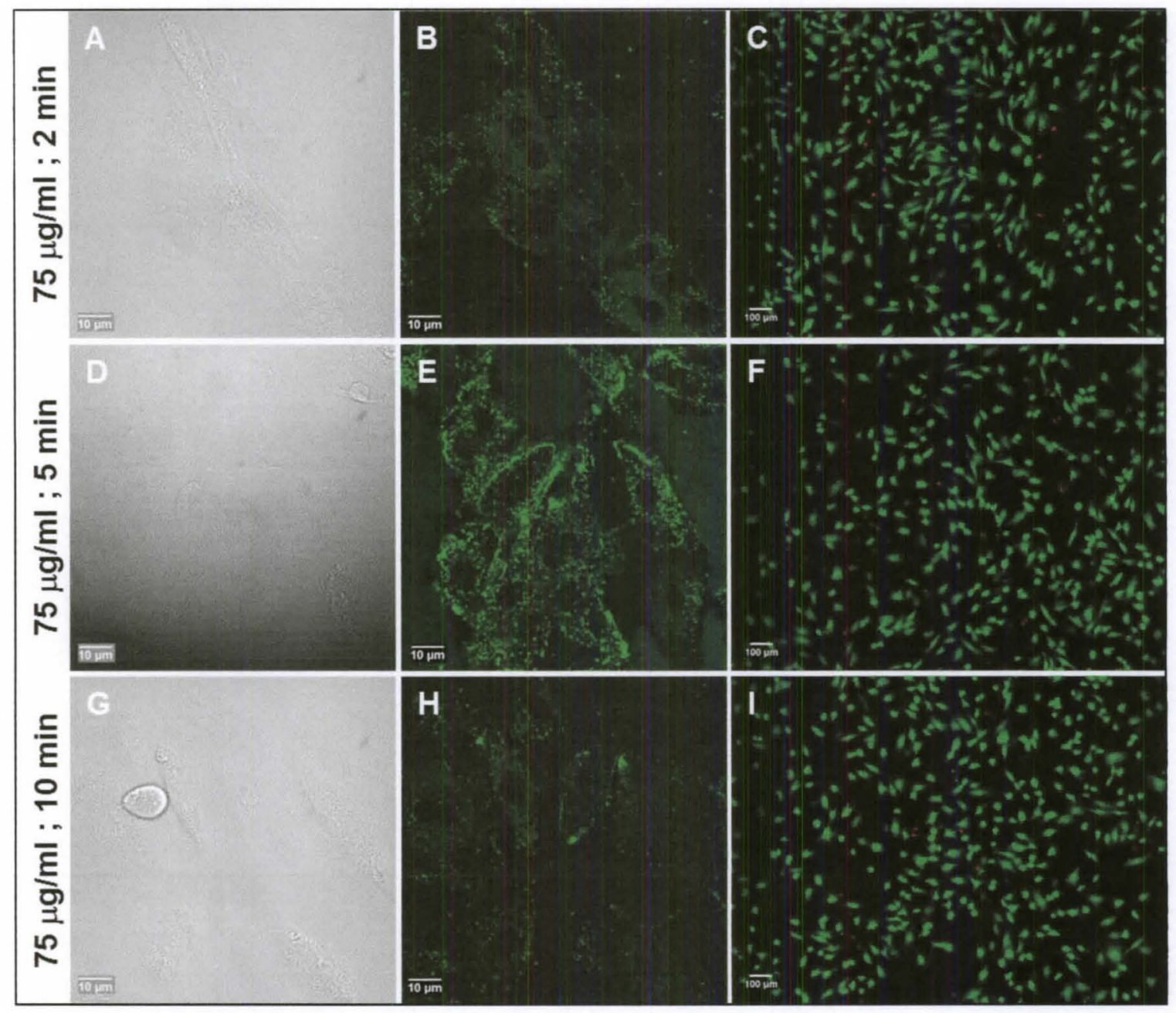

Figure 7.11 Reversible permeabilization using $75 \mu \mathrm{g} / \mathrm{ml}$ Saponin and cell viability. Comparison of bright field, fluorescence and viability representative images of $\mathrm{H} 9 \mathrm{C} 2$ cells treated with $25 \mu \mathrm{g} / \mathrm{ml}$ of Saponin for (A-C) 2 minutes, (D-F) 5 minutes and (G-I) 10 minutes respectively. Bright field and fluorescence images are obtained with $60 \times$ magnification and cell viability images were obtained with $10 \times$ magnification. Cell viability fluorescence images (C, F and I) show fluorescence overlay of calcein AM (green: live cells) and ethidium homodimer-1 (red: cells with damaged cell membrane). 


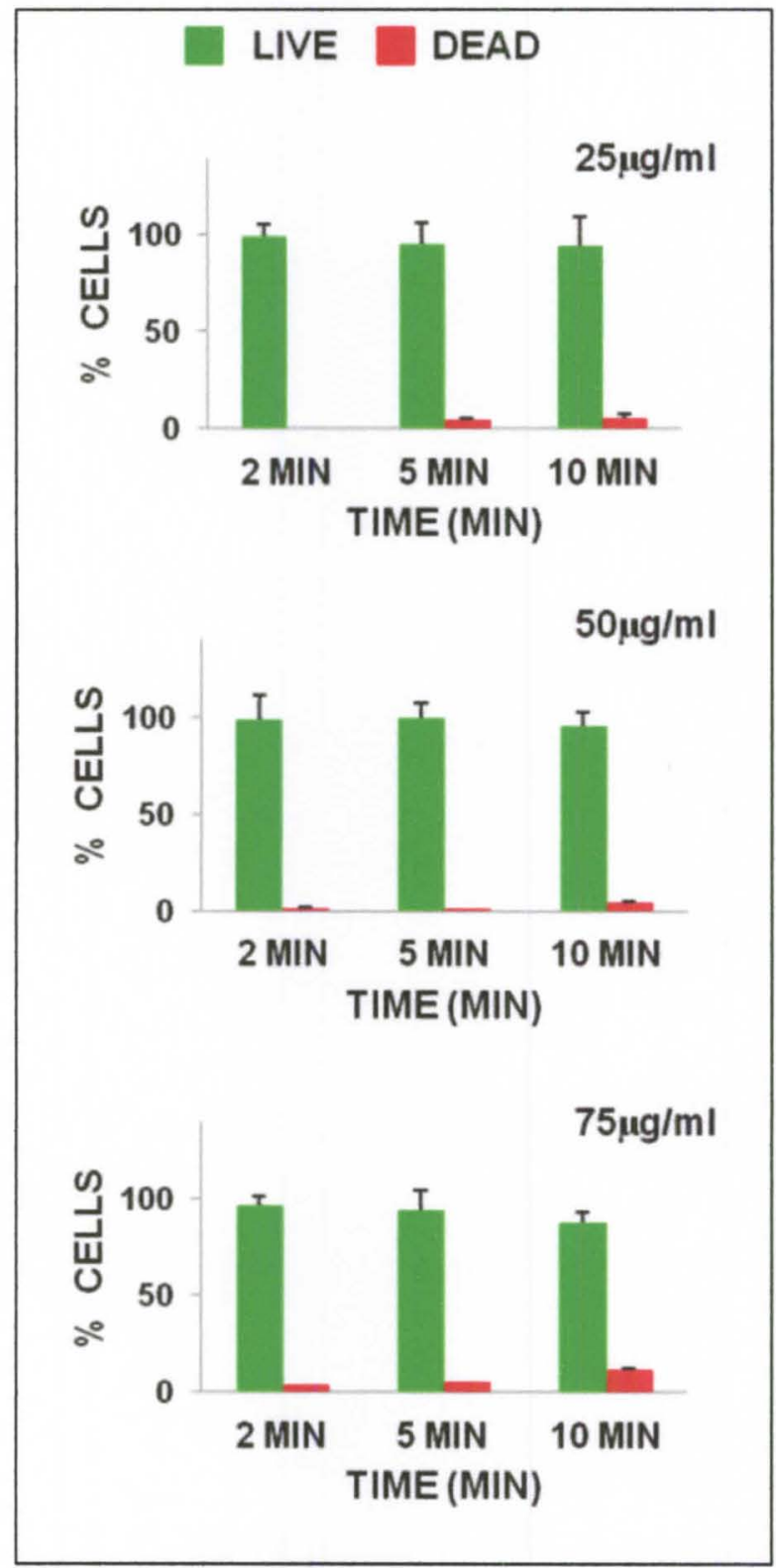

Figure 7.12 Live/Dead cell viability assay following Saponin treatment showing live cells stained with calcein AM (green: live cells) and dead cells stained with ethidium homodimer-1 (red: cells with damaged cell membrane). Cells are treated with different hypotonic (3:2) Saponin concentrations $(25 \mu \mathrm{g} / \mathrm{ml}, 50 \mu \mathrm{g} / \mathrm{ml}$ and $75 \mu \mathrm{g} / \mathrm{ml})$ for different incubation times $(2,5$ and 10 minutes $)$ at $4^{\circ} \mathrm{C}$ respectively. 


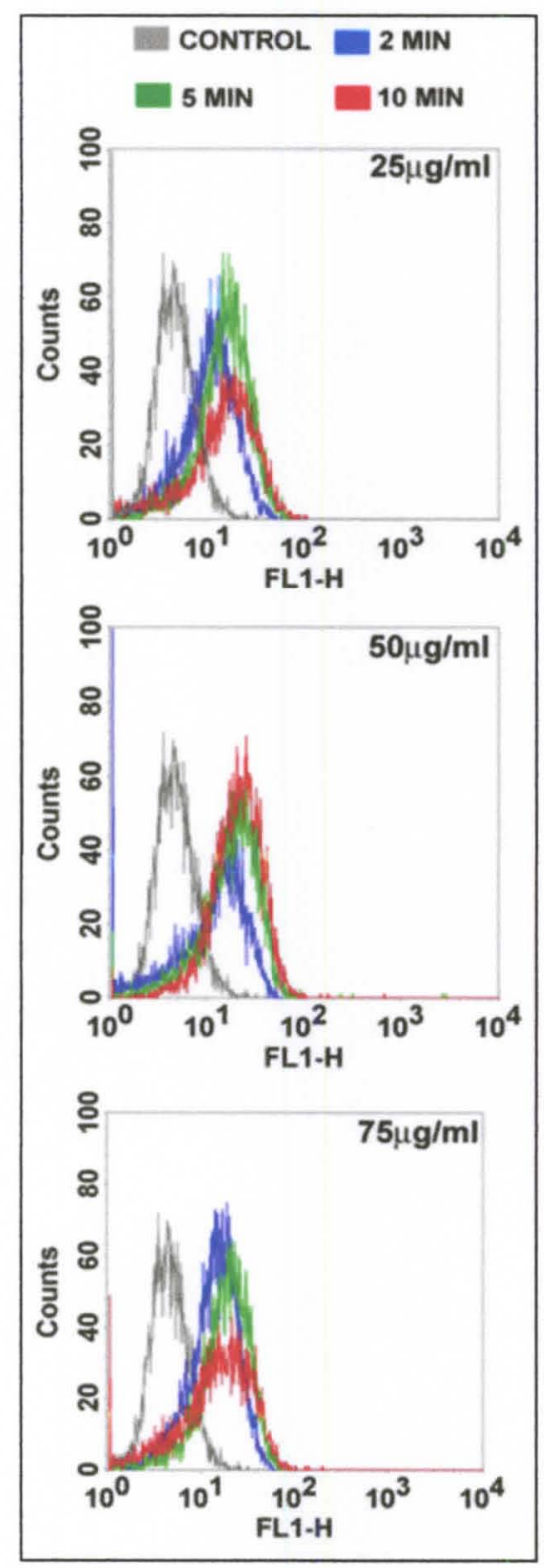

Figure 7.13 Quantitative analysis of internalized QDs with different Saponin concentrations. Histograms represent mean fluorescence intensity for $25 \mu \mathrm{g} / \mathrm{ml}(9.59$, $13.85,11.99), 50 \mu \mathrm{g} / \mathrm{ml}(10.21,16.43,19.38)$ and $75 \mu \mathrm{g} / \mathrm{ml}(13.32,16.97,14.03)$ for 2 minutes (blue), 5 minutes (green) and 10 minutes (red) respectively. The mean fluorescent intensity of cells without QDs used as negative control (gray) is 4.68. 
The G mean representing mean fluorescence intensity from the cells population for all the three different hypotonic Saponin conditions are $25 \mu \mathrm{g} / \mathrm{ml}(9.59,13.85,11.99)$, $50 \mu \mathrm{g} / \mathrm{ml}(10.21,16.43,19.38)$ and $75 \mu \mathrm{g} / \mathrm{ml}(13.32,16.97,14.03)$ for $2 \mathrm{~min}, 5 \mathrm{~min}$ and 10 min at $4^{\circ} \mathrm{C}$ respectively. Cells without QDs are used as negative control and have a mean fluorescence intensity of 4.68

\subsubsection{Reversible permeabilization using Saponin without hypotonic conditions}

The significance of hypotonic buffer conditions for the effective reversible permeabilization is evaluated by treating the H9c2 cells with Saponin in the absence of hypotonic buffer. The concentration of Saponin chosen for this study is $125 \mu \mathrm{g} / \mathrm{ml}$. Though this concentration is higher than the concentrations used with hypotonic buffer conditions (25-75 $\mu \mathrm{g} / \mathrm{ml}$ ), it is chosen due to the reason that lower concentrations of Saponin resulted in poor intracellular uptake of QDs. In other words, $125 \mu \mathrm{g} / \mathrm{ml}$ represents the lowest Saponin concentration where there is appreciable intracellular delivery of QDs. Even though, $125 \mu \mathrm{g} / \mathrm{ml}$ of Saponin results in the intracellular delivery of QDs, poor cell viability is observed (Figure 7.14). It is observed from confocal microscopy images (Figure 7.14.A) that the cell membranes are damaged due to the Saponin treatment.

To further confirm the importance of hypotonic buffer conditions, cells are evaluated for their viability using live/dead assay by treating cells with $125 \mu \mathrm{g} / \mathrm{ml}$ of Saponin without hypotonic buffer. The controls used in this study are cells alone without Saponin $(0 \mu \mathrm{g} / \mathrm{ml})$ as negative control and cells with Saponin $(1 \mathrm{mg} / \mathrm{ml})$ as positive control. 

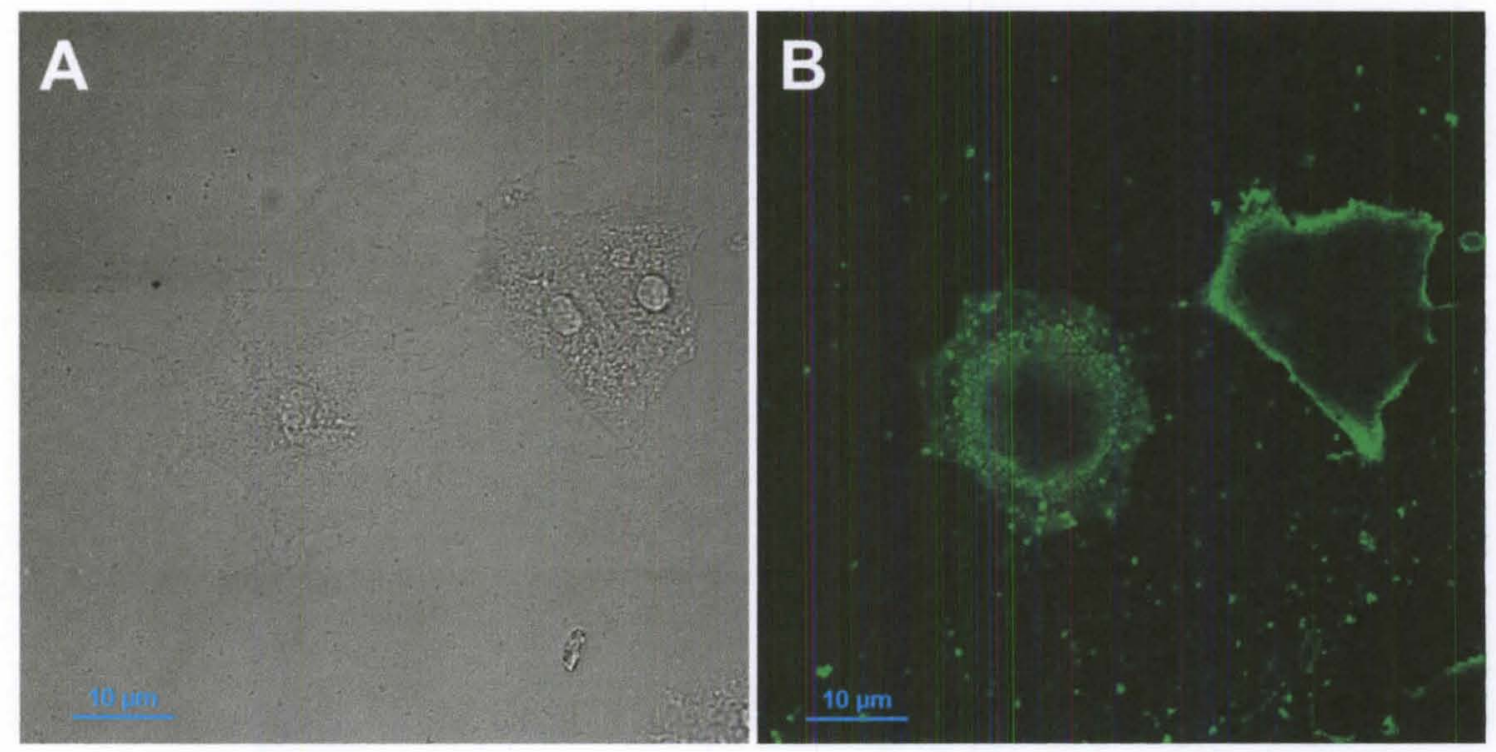

Figure 7.14 Reversible permeabilization using Saponin without hypotonic buffer conditions. (A) phase contrast, (B) fluorescence images of cells treated QDs mixed in $125 \mu \mathrm{g} / \mathrm{ml}$ of Saponin without hypotonic buffer.

Representative phase contrast and fluorescence overlay microscopy images from the live/dead assay for cells treated at different concentrations of Saponin, $0 \mu \mathrm{g} / \mathrm{ml}$ (Figure7.15.A, B) $125 \mu \mathrm{g} / \mathrm{ml}$ (Figure 7.15.C, D), $1 \mathrm{mg} / \mathrm{ml}$ (Figure 7.15.E, F) are shown. It is observed from the live/dead bright field images for $125 \mu \mathrm{g} / \mathrm{ml}$ and $1 \mathrm{mg} / \mathrm{ml}$ Saponin concentrations that the cell membranes are irreversibly damaged. The fluorescence images for these concentrations show that there is significant number of dead cells and minimal or no live cells as evident from the bright red (dead cells) and very low green (live cells) fluorescence. Quantitative analysis of the percentage of live/dead cells is performed by counting the number of live (green) and dead (red) cells from the cell viability images for a sample size of 5 (Figure 7.16). It is clearly evident that the $125 \mu \mathrm{g} / \mathrm{ml}$ Saponin 
concentration results in extensive cell death $(>98 \%)$ which is very similar to that of the positive control, $1 \mathrm{mg} / \mathrm{ml}$.

\subsection{DISCUSSION}

The efficient intracellular delivery of QDs is potentially very attractive in applications involving sub-cellular imaging as well as biomolecular transport to manipulate cell signaling mechanisms. Even though, several methods exist for the QDs intracellular delivery, most of them involve delivery of QDs as aggregates and not monodisperse. The only technique currently available which successfully demonstrates highly dispersed delivery of QDs is microinjection. However, this technique is very laborious and time consuming as the delivery is accomplished one cell at a time. Hence, an efficient method for the intracellular delivery of QDs which are freely dispersed in the cells without being trapped in the vesicles is needed. A novel method which overcomes the problems associated with the current techniques was developed in this dissertation. This technique combines the hypotonic exposure with low concentrations of a cell permeabilization agents like Saponin to accomplish reversible permeabilization of cell membranes for QDs delivery. Performing this protocol at $4{ }^{\circ} \mathrm{C}$ inhibits thermal energy dependent mechanisms like endocytosis. Therefore, it ensures that uptake of QDs is primarily due to this reversible permeabilization technique. 


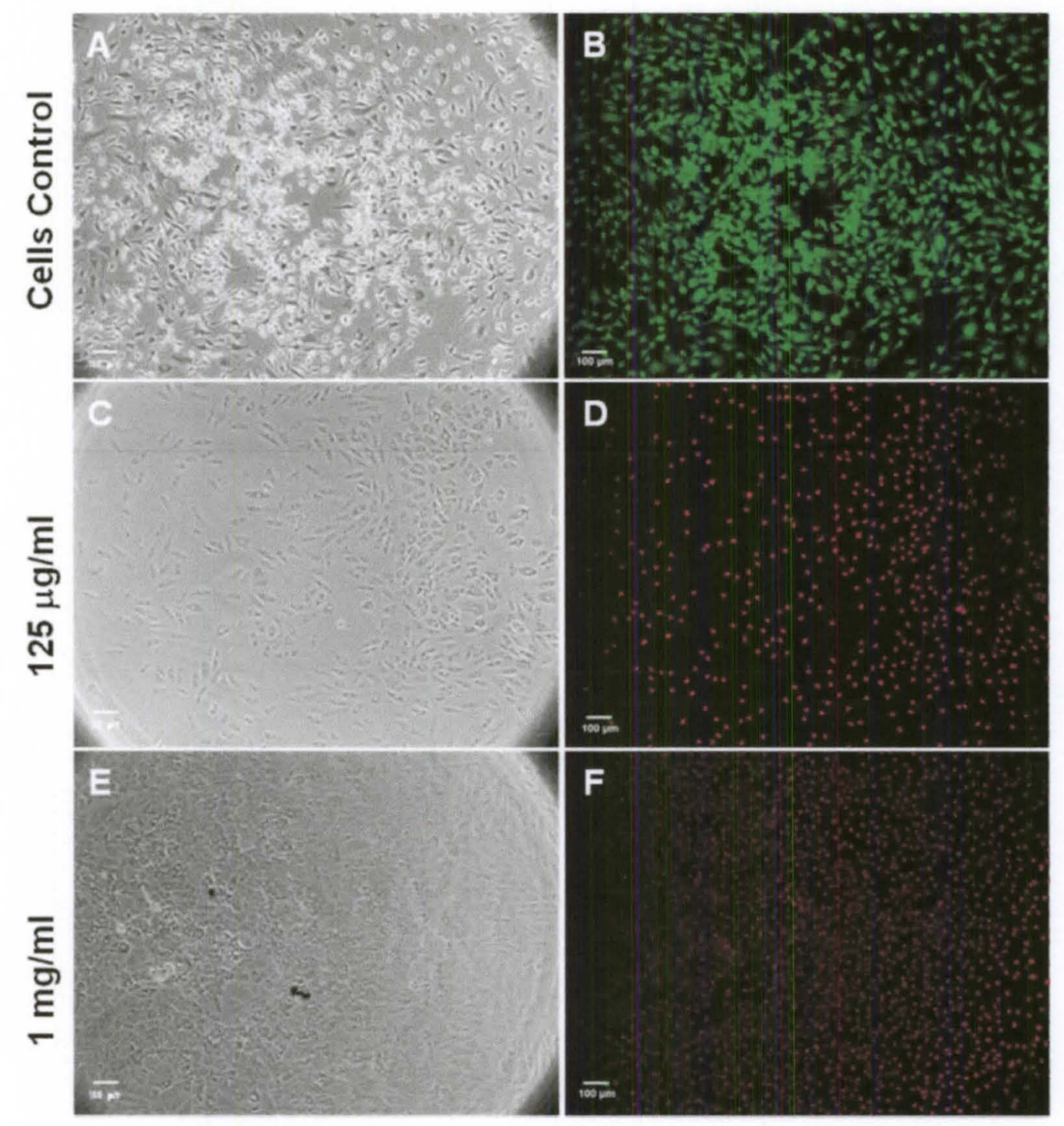

Figure 7.15 Permeabilization using Saponin without hypotonic conditions. Comparison of bright field and fluorescence overlay images of calcein AM (green: live cells) and ethidium homodimer-1 (red: cells with damaged cell membrane) co-stained H9c2 cells treated with (A, B) $0 \mu \mathrm{g} / \mathrm{ml}$ Saponin, cells only (negative control), (C, D) $125 \mu \mathrm{g} / \mathrm{ml}$ Saponin without hypotonic buffer, (E, F) $1 \mathrm{mg} / \mathrm{ml}$ Saponin without hypotonic buffer (positive control) respectively. 


\section{LIVE D DEAD}

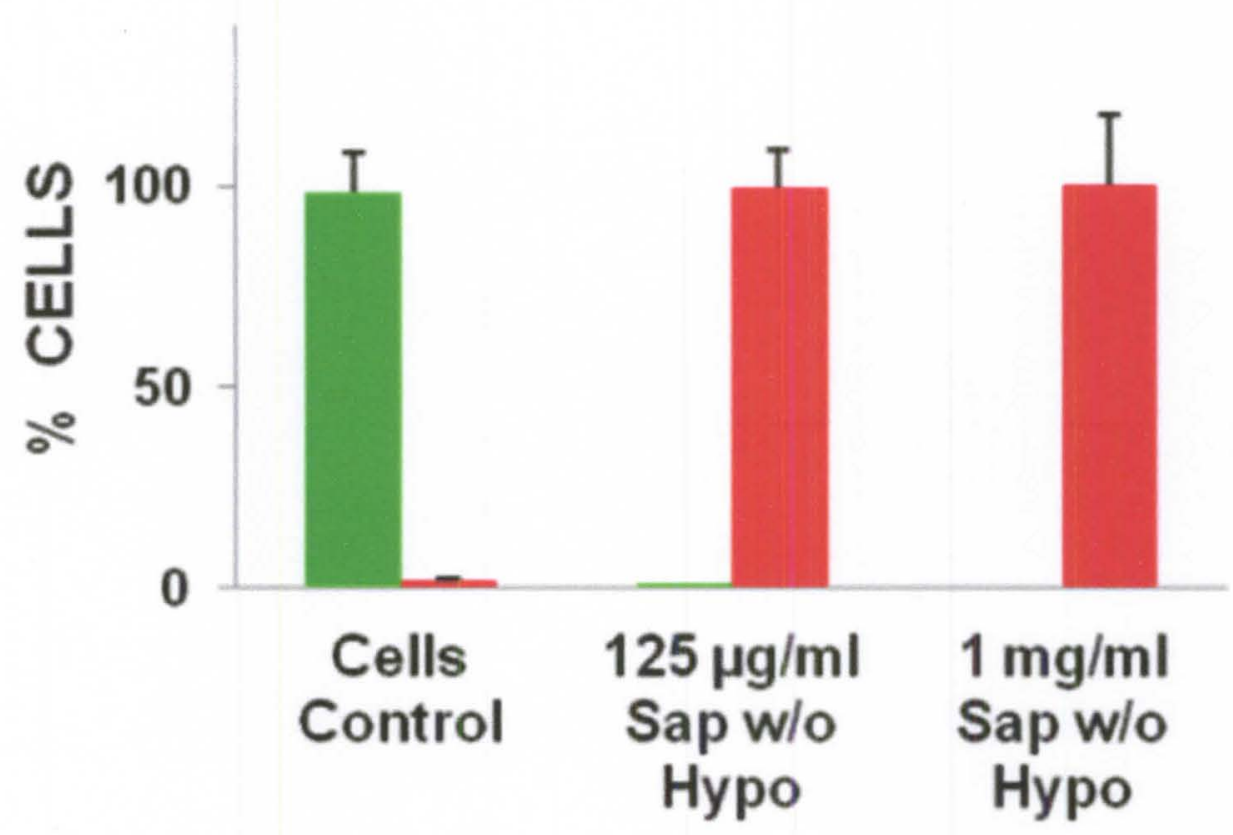

Figure 7.16 Quantitative analysis of live/dead cell assay performed on cells treated with Saponin without hypotonic buffer conditions. Comparison of percentage of live/dead cells stained with of calcein AM (green: live cells) and ethidium homodimer-1 (red: cells with damaged cell membrane) is shown for $0 \mu \mathrm{g} / \mathrm{ml}$ Saponin i.e., cells alone (negative control), $125 \mu \mathrm{g} / \mathrm{ml}$ Saponin without hypotonic buffer and $1 \mathrm{mg} / \mathrm{ml}$ Saponin without hypotonic buffer (positive control) respectively.

It is observed that hypotonic exposure alone is sufficient for reversible permeabilization of live cells for the delivery of QDs as seen from the Figures 7.4, 7.5 and 7.6. Furthermore, the cells treated with different hypotonic buffer conditions are show high viability. It is experimentally determined that a 5min exposure of QDs to 3:2 buffer to DI water results in uniform distribution of the cell internalized QDs. Moreover, 
hypotonic exposure in combination with Saponin treatment results in more efficient and highly dispersed delivery of QDs. The mean fluorescence intensities are a measure of the number of QDs inside the cells while the fluorescence images compare the distribution of QDs within the cells. It was experimentally determined that a 5 min exposure to QDs suspended in 3:2 buffer to DI water ratio with $50 \mu \mathrm{g} / \mathrm{ml}$ Saponin produced the best dispersion of the internalized QDs within the cytoplasm. Cell viability was verified from the live/dead assay which shows that this reversible permeabilization technique maintains high cell viability. The viability study was performed after 30-45 min to be consistent with the amount of time between recovery and imaging the cells with internalized QDs. The live/dead viability assay is performed without QDs loading. This was due to the fact that calcein-AM which is used to label live cells has emission around $517 \mathrm{~nm}$ which interferes with QDs fluorescence emission ( $\sim 530 \mathrm{~nm})$. Moreover, it was observed that the cells with internalized QDs continued to divide and proliferate even after several days without any apparent loss of viability.

The significance of hypotonic buffer conditions is twofold. First, it creates an osmotic pressure gradient that drives fluid into the cell. This fluid transport results in the intracellular of delivery of QDs. Second, it ensures unidirectional transport directed into the cells. The cells contain various biomolecules and other important intracellular contents which can diffuse out of the cells either during the creation of openings. This efflux results in cell death and loss of proper function. Further, this bidirectional transport is highly critical when the cells are treated with Saponin as it creates holes in the membrane. Thus, the hypotonic buffer environment maintains the osmotic pressure gradient which ensures cell viability and no net loss of vital intracellular contents. It is also observed that during 
reversible permeabilization using hypotonic buffer, $10 \mathrm{~min}$ exposure to hypotonic conditions, did not significantly enhance the number of internalized QDs. This can be explained from the fact that after $5 \mathrm{~min}$ the extracellular and intracellular environments equilibrate with each other. This results in reduction in the pressure gradient and fluid transport into the cell and diffusion occurs both into and out of the cells. Therefore, the $10 \mathrm{~min}$ exposure time does not significantly enhance the number of internalized QDs as seen from the mean fluorescence intensities from the flow cytometry analysis.

To further investigate the significance of hypotonic buffer conditions, experiments were performed with Saponin in the absence of a hypotonic environment. The low concentrations of Saponin used in combination without hypotonic buffer $(25-75 \mu \mathrm{g} / \mathrm{ml})$ did not result in a significant uptake of QDs by the cells. Only when the concentration of Saponin is raised to $125 \mu \mathrm{g} / \mathrm{ml}$, an appreciable number of QDs are seen to be internalized by the cells. Extensive cell death is observed when the cells are treated with $125 \mu \mathrm{g} / \mathrm{ml}$ of Saponin for $5 \mathrm{~min}$ without hypotonic conditions from the live/dead assay. This could possibly be due to the leakage of vital intracellular contents out of the cell in the absence of an osmotic pressure gradient across the membrane. Thus, the hypotonic exposure is necessary to maintain cell viability during reversible permeabilization using Saponin.

Thus this reversible permeabilization technique using low concentrations of Saponin with hypotonic buffer conditions is a highly efficient method for delivery of QDs free in the cellular cytoplasm. The viability of the cells is not compromised following the Saponin or hypotonic treatments. Despite the fact that all the experiments in this study were performed using adherent cells, this technique is highly applicable for cells in suspension. As Saponin targets cholesterol rich membranes, the delivery of QDs is highly 
selective towards cytoplasmic delivery than nuclear delivery as clearly evident from the confocal fluorescence images. Moreover, the osmotic pressure gradient is across the cell membrane. This technique of reversible permeabilization using hypotonic Saponin is applicable in in vitro studies such as labeling of the different intracellular components and tracking of proteins and other subcellular biomolecules. The delivery of QDs based on hypotonic buffer conditions alone is applicable in in vivo studies such as labeling of blood leukocytes by selective lysis of red blood cells. Thus, this reversible permeabilization of live cells technique wide applications in the delivery of QDs into cells for applications such as imaging and biomolecular delivery. Furthermore, this technique can potentially be extended to delivery of various other nanomaterials and also biomolecules into live cells.

\subsection{ODS: NF-kB P65 ANTIBODY CONJUGATES}

This section deals with the preliminary results obtained from study to demonstrate QDs conjugated NF-kB p65 antibodies have the ability to image the intracellular location of NF-kB protein. QDs are conjugated to NF-kB p65 antibodies and gel electrophoresis is performed (Ocean Nanotech LLC) to confirm the conjugation of NF-kB p65 antibodies to QDs. It is observed from the gel electrophoresis data that the unconjugated QDs migrate faster than the antibody conjugated QDs (Figure 7.17). QDs with $\sim 600 \mathrm{~nm}$ emission are conjugated with NF-kB p65 antibodies using protein-G attachment. Gel electrophoresis is used to confirm the conjugation of QDs to protein-G and QDs: $\mathrm{pG}$ conjugates to NF-kB p65 antibodies (Figure 7.18). The tailing observed is due to the different number of antibodies attached to each QD. 


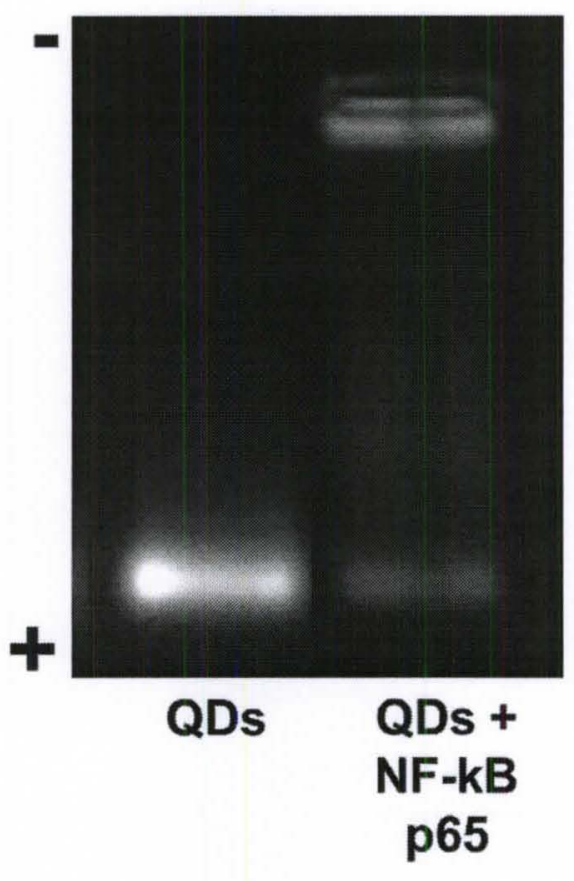

Figure 7.17 Gel electrophoresis data confirming the conjugation of QDs with NF-kB p65 antibodies. Tailing is due to the different number of antibodies attached to each QD. 


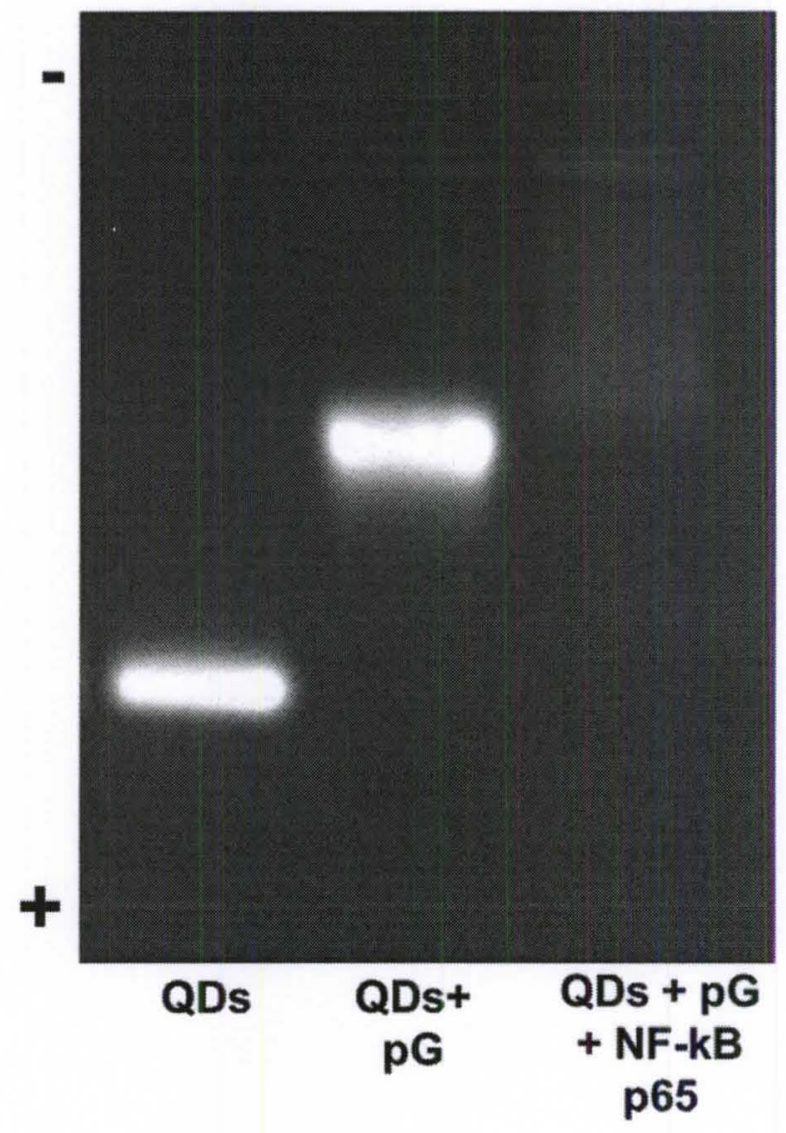

Figure 7.18 Gel electrophoresis data confirming the conjugation of QDs with protein G and with NF-kB p65 antibodies.

\subsubsection{Stimulation of cells for activation and nuclear translocation of NF-kB}

In addition to gel electrophoresis, the most definitive method to confirm the bioconjugation of QDs is by performing a functional assay by testing the avidity of antibody to its functional protein. The results shown in this section for labeling of NF-kB protein is accomplished using QDs with emission $\sim 530 \mathrm{~nm}$ and $\sim 600 \mathrm{~nm}$. Cells are stimulated for the activation and translocation of NF-kB protein from the cytoplasm to the nucleus. Cells stimulation is performed using stimuli like LPS (2 hours), TNF- $\alpha$ (1 hour) and UV radiation (2 hours). Following stimulation, immunofluorescence staining of 
NF-kB protein is performed using QDs: NF-kB p65 conjugates. Confocal fluorescence imaging of the cells revealed strong QDs fluorescence emerging from the nucleus for the UV (Figure 7.19B), TNF- $\alpha$ (Figure 7.19C) and LPS (Figure 7.19D) stimulated cells. This indicates successful labeling of nuclear NF-kB protein using the QDs: NF-kB p65 conjugates. The cells with strong nuclear fluorescence in the represented field of view are shown (arrows). The TNF- $\alpha$ stimulated cells are stained for NF-kB using NF-kB p65 antibodies conjugated to QDs with $\sim 600 \mathrm{~nm}$ emission. In the case of un-stimulated cells (negative control), there is minimal nuclear fluorescence and only cytoplasmic fluorescence signal (Figure 7.19A). This is expected as the cells are not stimulated, there is no nuclear translocation of NF-kB protein.

\subsection{SUMMARY}

In summary, the results shown in this chapter demonstrate the novel technique to efficiently delivery QDs well dispersed in live cells. This technique combines the use of hypotonic environments and low concentrations of cell permeabilization agents like Saponin. Enhanced and well dispersed delivery of QDs is achieved when Saponin was used in combination with hypotonic conditions in comparison to hypotonic conditions alone. The ability of QDs to label subcellular proteins like NF-kB using QDs: NF-kB p65 conjugates is also demonstrated. The reversible permeabilization technique can potentially be used to track the nuclear translocation of NF-kB in live cells which provides vital information regarding the activity of the protein. The next chapter discusses the conclusions and potential future directions based on this dissertation research. 


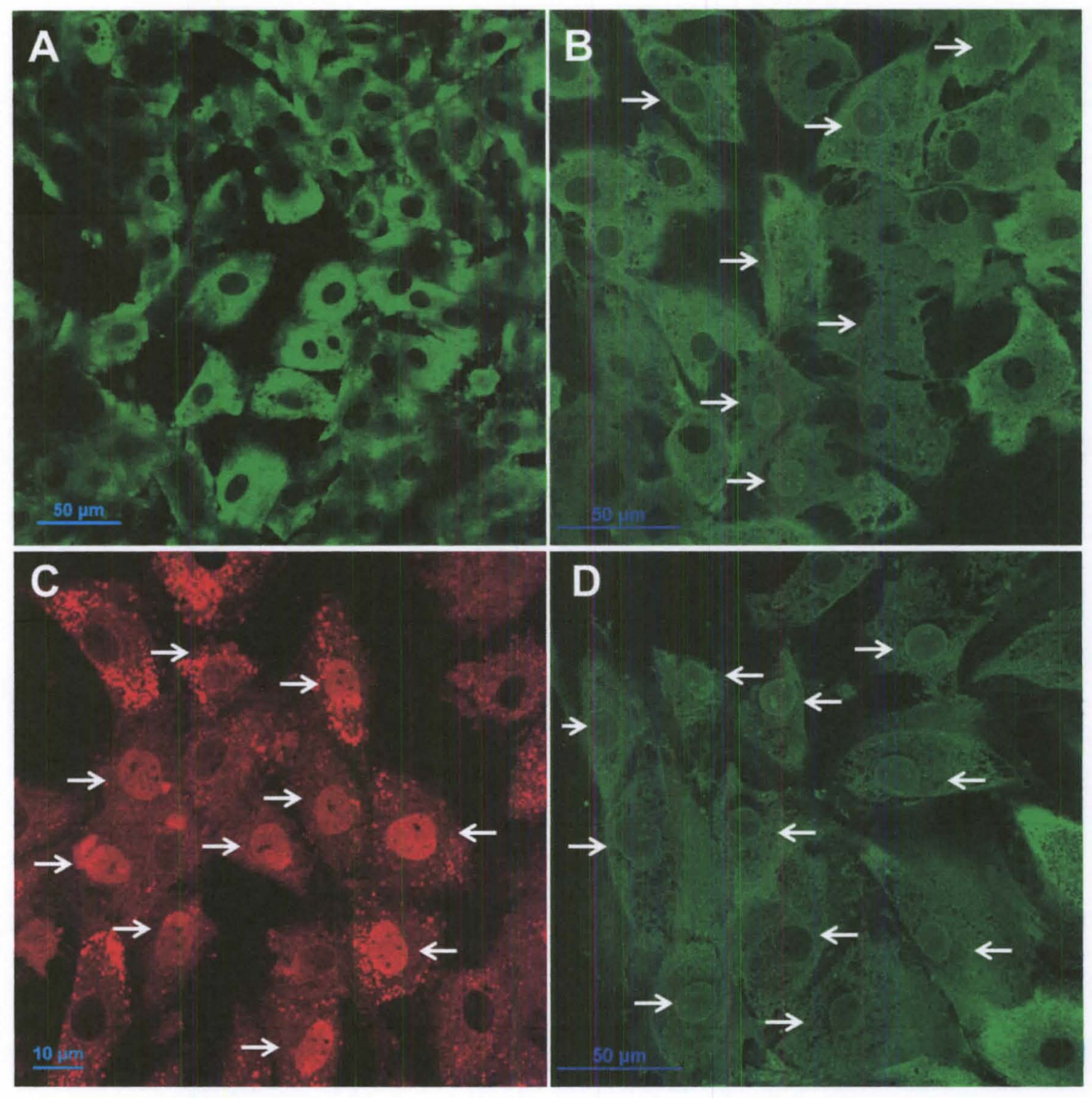

Figure 7.19 Stimulation of cells for activation and nuclear translocation of NF-kB protein. Immunofluorescence on cells stained for NF-kB protein using QDs: NF-kB p65 antibodies for (A) unstimulated cells, (B) UV radiation, (C) TNF- $\alpha$ and (D) LPS stimulated cells respectively. The arrows (white) are used to identify cells with nuclear fluorescence. The TNF- $\alpha$ stimulated cells are stained with QDs with $\sim 600 \mathrm{~nm}$ emission. All the images are taken with a magnification of $60 x$. 


\section{CHAPTER VIII- CONCLUSIONS AND FUTURE DIRECTIONS}

\subsection{CONCLUSIONS}

This dissertation research involves the use of two different nanomaterials for the delivery well as imaging of the biomolecules with subcellular resolution. The dissertation is divided into two parts - chapters III-V as first part and c chapters V-VII as the second part.

In the first part, the biocompatibility of SWCNTs is thoroughly evaluated by studying their interactions with the leukocytes in the blood. Blood is chosen as the appropriate model as it is a living tissue and is a major mediator of immune homeostasis. Two different interactions are recreated in vitro. In the direct interaction, the presence of SWCNTs in blood circulation is studied by evaluating the response of leukocytes to SWCNTs. In the indirect interaction, the presentation of SWCNTs to the blood leukocytes via antigen presenting cells (APCs) is studied. The different subpopulations of the blood leukocytes are identified using their respective phenotype markers and are evaluated for their activation using their respective activation markers. Flow cytometry is used to analyze the different subpopulations for any activation relative to controls.

Results from this study conclusively indicate minimal leukocyte activation following both the direct and the indirect interactions with SWCNTs. The delivery of ssDNA into cells using SWCNTs is also demonstrated. These results 
provide new information regarding the biocompatibility of nanotubes and support the use of CNTs based approaches for intracellular delivery of genes and biomolecules.

In the second part of this dissertation, a new technique is demonstrated that addresses common problems with nanomaterial delivery into cells like confinement in vesicles and delivery as aggregates. It combines the use of a hypotonic buffer in combination with low concentrations of a cell permeabilization agent, Saponin to accomplish reversible permeabilization of cells for delivery of QDs. This technique is comparable to microinjection technique that has the highest success in intracellular QD delivery with significantly higher throughput. The cell viability following this technique is high and cells survive in culture for extended periods of time undergoing cell division. This technique has great potential for delivery of QDs for both imaging and biomolecular delivery applications. The preliminary results showing the labeling of an important intracellular protein (NF-kB) using QDs is demonstrated.

\subsection{FUTURE DIRECTIONS}

The thorough study performed to evaluate the biocompatibility of SWCNTs paves way for their safe use in the delivery of therapeutics, cancer diagnostics and tissue engineering applications. The SWCNTs can be further functionalized with other relevant biomolecules like nucleic acids, proteins or peptides for clinical applications. The reversible permeabilization technique is easily applicable in the endocytosis free delivery of not only QDs but also other nanomaterials. The technique accomplishes delivery of nanomaterials in live cells and therefore has the potential to study intracellular processes in real time. The nanomaterials based approaches described can be easily integrated into 
state of the art technologies utilizing microfluidic platforms for more efficient drug delivery and disease diagnosis. 


\section{REFERENCES}

1. Feynman, R. in American Physical Society California Insitute of Technology, Pasadena 1960).

2. Hobson, D.W. Commercialization of nanotechnology. Wiley Interdiscip Rev Nanomed Nanobiotechnol 1, 189-202 (2009).

3. Paull, R., Wolfe, J., Hebert, P. \& Sinkula, M. Investing in nanotechnology. Nat Biotechnol 21, 1144-1147 (2003).

4. Firkowska, I.G., Suna Rojas-Chapana, José A. Luecke, Klaus Brüstle, Oliver Giersig, Michael in Nanomaterials for Application in Medicine and Biology. (ed. M.K. Giersig, Gennady B.) 1-15 (Springer Netherlands, 2008).

5. Nie, S., Xing, Y., Kim, G.J. \& Simons, J.W. Nanotechnology Applications in Cancer. Annual Review of Biomedical Engineering 9, 257-288 (2007).

6. Saito, N. et al. Carbon nanotubes: biomaterial applications. Chemical Society Reviews 38, 1897-1903 (2009).

7. Ragusa, A.Z., Antonella Aloisi, Alessandra Pellegrino, Teresa 257-311, 224 (CRC Press, 2011).

8. Wagner, V.D., Anwyn Bock, Anne-Katrin Zweck, Axel The emerging nanomedicine landscape. Nat Biotech 24, 1211-1217 (2006).

9. Sollid, L.M. Molecular Basis of Celiac Disease. Annual Review of Immunology 18, 53-81 (2000). 
10. Zimmermann, G.R., Lehár, J. \& Keith, C.T. Multi-target therapeutics: when the whole is greater than the sum of the parts. Drug Discovery Today 12, 34-42 (2007).

11. Opalinska, J.B. \& Gewirtz, A.M. Nucleic-acid therapeutics: basic principles and recent applications. Nat Rev Drug Discov 1, 503-514 (2002).

12. Gannon, C.J., Patra, C.R., Bhattacharya, R., Mukherjee, P. \& Curley, S.A. Intracellular gold nanoparticles enhance non-invasive radiofrequency thermal destruction of human gastrointestinal cancer cells. Journal of Nanobiotechnology 6, 2 (2008).

13. Roszek B, J.W.d., Geertsma RE (National Institute for Public Health and the Environment, 2005).

14. Salata, O. Applications of nanoparticles in biology and medicine. Journal of Nanobiotechnology 2, 3 (2004).

15. Sarojini, H., Medepalli, K., Terry, D.A., Alphenaar, B.W. \& Wang, E. Localized delivery of DNA to the cells by viral collagen-loaded silica colloidal crystals. Biotechniques 43, 213-214, 216-218, 220-211 (2007).

16. Freitas, R.A. Current status of nanomedicine and medical nanorobotics. $J$ Comput Theor Nanos 2, 1-25 (2005).

17. S.Iijima Helical microtubulues of graphitic carbon. Nature 354, 56-58 (1991).

18. Gooding, J.J. Nanostructuring electrodes with carbon nanotubes: A review on electrochemistry and applications for sensing. Electrochimica Acta 50, 3049-3060 (2005).

19. Trojanowicz, M. Analytical applications of carbon nanotubes: a review. $\operatorname{Tr} A C$ Trends in Analytical Chemistry 25, 480-489 (2006).

20. Gong, K. et al. Electrochemistry and Electroanalytical Applications of Carbon Nanotubes: A Review. Analytical Sciences 21, 1383-1393 (2005). 
21. Cao, Q. \& Rogers, J.A. Ultrathin Films of Single-Walled Carbon Nanotubes for Electronics and Sensors: A Review of Fundamental and Applied Aspects. Advanced Materials 21, 29-53 (2009).

22. Avouris, P. Molecular Electronics with Carbon Nanotubes. Accounts of Chemical Research 35, 1026-1034 (2002).

23. McEuen, P.L., Fuhrer, M.S. \& Hongkun, P. Single-walled carbon nanotube electronics. Nanotechnology, IEEE Transactions on 1, 78-85 (2002).

24. Kempa, K. et al. Photonic Crystals Based on Periodic Arrays of Aligned Carbon Nanotubes. Nano Letters 3, 13-18 (2002).

25. Sun, Y.-P., Fu, K., Lin, Y. \& Huang, W. Functionalized Carbon Nanotubes: $\square$ Properties and Applications. Accounts of Chemical Research 35, 1096-1104 (2002).

26. Yu, M.-F., Files, B.S., Arepalli, S. \& Ruoff, R.S. Tensile Loading of Ropes of Single Wall Carbon Nanotubes and their Mechanical Properties. Physical Review Letters 84, 5552 (2000).

27. Dyke, C.A. \& Tour, J.M. Covalent Functionalization of Single-Walled Carbon Nanotubes for Materials Applications. The Journal of Physical Chemistry A 108, 11151-11159 (2004).

28. Davis, M.E. Ordered porous materials for emerging applications. Nature 417, 813-821 (2002).

29. Bianco, A., Kostarelos, K. \& Prato, M. Applications of carbon nanotubes in drug delivery. Current Opinion in Chemical Biology 9, 674-679 (2005).

30. Prato, M., Kostarelos, K. \& Bianco, A. Functionalized Carbon Nanotubes in Drug Design and Discovery. Accounts of Chemical Research 41, 60-68 (2007).

31. Klumpp, C., Kostarelos, K., Prato, M. \& Bianco, A. Functionalized carbon nanotubes as emerging nanovectors for the delivery of therapeutics. Biochimica et Biophysica Acta (BBA) - Biomembranes 1758, 404-412 (2006). 
32. Balaji, P. \& Eric, W. Nanotechnology for Sensing, Imaging, and Treating Cancer. Surgical oncology clinics of North America 16, 293-305 (2007).

33. Teker, K. et al. Applications of carbon nanotubes for cancer research. NanoBioTechnology 1, 171-182 (2005).

34. Correa-Duarte, M.A. et al. Fabrication and Biocompatibility of Carbon NanotubeBased 3D Networks as Scaffolds for Cell Seeding and Growth. Nano Letters 4, 2233-2236 (2004).

35. Hu, H., Ni, Y., Montana, V., Haddon, R.C. \& Parpura, V. Chemically Functionalized Carbon Nanotubes as Substrates for Neuronal Growth. Nano Letters 4, 507-511 (2004).

36. Bhadeshia, H.K.D.H. $52^{\text {nd }}$ Hatfield Memorial lecture large chunks of very strong steel. Materials Science \& Technology 21, 1293-1302 (2005).

37. Dai, H. Carbon Nanotubes. (Springer:Berlin, 2001).

38. Iijima, S., Ichihashi, Toshinari Single-shell carbon nanotubes of 1-nm diameter. Nature 363, 603-605 (1993).

39. Bethune, D.S., Klang, C. H.,de Vries, M. S.,Gorman, G.,Savoy, R.,Vazquez, J.,Beyers, R. Cobalt-catalysed growth of carbon nanotubes with single-atomiclayer walls. Nature 363, 605-607 (1993).

40. Ebbesen, T.W., Ajayan, P. M. Large-scale synthesis of carbon nanotubes. Nature 358, 220-222 (1992).

41. Thess Andreas Lee, R., Nikolaev, Pavel,Dai, Hongjie,Petit, Pierre,Robert, Jerome,Xu, Chunhui,Lee, Young Hee,Kim, Seong Gon,Rinzler, Andrew G.,Colbert, Daniel T.,Scuseria, Gustavo E.,Tomanek, David,Fischer, John E.,Smalley, Richard E. Crystalline Ropes of Metallic Carbon Nanotubes. Science 273, 483-487 (1996).

42. Saito, R., Fujita, M., Dresselhaus, G. \& Dresselhaus, M.S. Electronic-Structure of Chiral Graphene Tubules. Applied Physics Letters 60, 2204-2206 (1992). 
43. Dresselhaus M.S, D., G. Eklund, P. C. Science of Fullerenes and Carbon Nanotubes. (Academic, San Diego; 1996).

44. R.Saito, M.D., G.Dresselhaus. Physical properties of carbon nanotubes. (Imperial College Press 1998).

45. M.Dresselhaus, G., Dresselhaus,Ph.Avouris Carbon Nanotubes: Synthesis, Structure, Properties and Applications, Edn. 1. (Springer, 2001).

46. S.Datta From atoms to transistors (Cambridge University Press, 2005).

47. Ajayan, P.M., Charlier, J.-C. \& Rinzler, A.G. Carbon nanotubes: From macromolecules to nanotechnology. Proceedings of the National Academy of Sciences 96, 14199-14200 (1999).

48. Mark P. Mattson, R.C.H., Apparao M. Rao Molecular functionalization of carbon nanotubes and use as substrates for neuronal growth Journal of Molecular Neuroscience 14, 175-182 (2000).

49. Baughman, R.H., Cui, Changxing,Zakhidov, Anvar A.,Iqbal, Zafar,Barisci, Joseph N.,Spinks, Geoff M.,Wallace, Gordon G.,Mazzoldi, Alberto,De Rossi, Danilo,Rinzler, Andrew G.,Jaschinski, Oliver,Roth, Siegmar,Kertesz, Miklos Carbon Nanotube Actuators. Science 284, 1340-1344 (1999).

50. Bahr, J.L. \& Tour, J.M. Covalent chemistry of single-wall carbon nanotubes. Journal of Materials Chemistry 12, 1952-1958 (2002).

51. Niyogi, S. et al. Chemistry of single-walled carbon nanotubes. Accounts of Chemical Research 35, 1105-1113 (2002).

52. Hirsch, A. Functionalization of Single-Walled Carbon Nanotubes. Angewandte Chemie International Edition 41, 1853-1859 (2002).

53. Sun, Y.P., Fu, K., Lin, Y. \& Huang, W. Functionalized carbon nanotubes: properties and applications. Accounts of Chemical Research 35, 1096-1104 (2002). 
54. Wenrong Yang, P.T., J Justin Gooding,Simon P Ringer,Filip Braet Carbon nanotubes for biological and biomedical applications. Nanotechnology 18 (2007).

55. Liu, J., Rinzler, Andrew G., Dai, Hongjie,Hafner, Jason H, Bradley, R. Kelley,Boul, Peter J.,Lu, Adrian,Iverson, Terry,Shelimov, Konstantin,Huffman, Chad B.,Rodriguez-Macias, Fernando,Shon, ,Young-Seok, Lee, T. Randall,Colbert, Daniel T.,Smalley, Richard E. Fullerene Pipes. Science 280, 1253-1256 (1998).

56. ShikChi Tsang, Z.G., Yao, Kuan Chen, Malcolm L. H. Green, H. Allen O. Hill ,Trevor W. Hambley, Peter J. Sadler Immobilization of Platinated and Iodinated Oligonucleotides on Carbon Nanotubes. Angewandte Chemie International Edition in English 36, 2198-2200 (1997).

57. Zijian Guo, P.J.S.S.C.T. Immobilization and Visualization of DNA and Proteins on Carbon Nanotubes. Advanced Materials 10, 701-703 (1998).

58. Zheng Ming, J.A., Semke Ellen D,Diner Bruce A,McLean Robert S,Lustig Steve R,Richardson Raymond E,Tassi Nancy G. DNA-assisted dispersion and separation of carbon nanotubes. Nat Mater 2, 338-342 (2003).

59. Foldvari, M. \& Bagonluri, M. Carbon nanotubes as functional excipients for nanomedicines: I. pharmaceutical properties. Nanomedicine: Nanotechnology, Biology and Medicine 4, 173-182 (2008).

60. Kam, N.W.S., Liu, Z. \& Dai, H. Carbon Nanotubes as Intracellular Transporters for Proteins and DNA: An Investigation of the Uptake Mechanism and Pathway. Angewandte Chemie 118, 591-595 (2006).

61. Bianco, A.K., Kostas Prato, Maurizio Applications of carbon nanotubes in drug delivery. Current Opinion in Chemical Biology 9, 674-679 (2005).

62. Jin, H., Heller, D.A., Sharma, R. \& Strano, M.S. Size-Dependent Cellular Uptake and Expulsion of Single-Walled Carbon Nanotubes: Single Particle Tracking and a Generic Uptake Model for Nanoparticles. ACS Nano 3, 149-158 (2009).

63. Zhuang Liu, M.W., Mark Holodniy,Hongie Dai siRNA Delivery into Human T Cells and Primary Cells with Carbon-Nanotube Transporters. Nanobiotechnology 46 (2007). 
64. Hu H.Ni Yingchun, M.V., Haddon, R. C. Parpura, V. Chemically Functionalized Carbon Nanotubes as Substrates for Neuronal Growth. Nano Lett. 4, 507-511 (2004).

65. Xu, Y., Pehrsson, P.E., Chen, L., Zhang, R. \& Zhao, W. Double-Stranded DNA Single-Walled Carbon Nanotube Hybrids for Optical Hydrogen Peroxide and Glucose Sensing. J. Phys. Chem. C 111, 8638-8643 (2007).

66. O'Connell, M.J.B., S. M. Huffman, C. B. Moore, V. C. Strano, M. S. Haroz, E. H. Rialon, K. L. Boul, P. J. Noon, W. H. Kittrell, C. Ma, J. Hauge, R. H. Weisman, R. B. Smalley, R. E. Band gap fluorescence from individual single-walled carbon nanotubes. Science 297, 593-596 (2002).

67. Heller DA, B.S., Eurell TE, Strano MS Single-walled carbon nanotube spectroscopy in live cells: Towards long-term labels and optical sensors. $A d v$ Mater 17, 2793-2799 (2005).

68. Nadine Wong Shi Kam, M.O.C., Jeffrey A. Wisdom,Hongjie Dai Carbon nanotubes as multifunctional biological transporters and near-infrared agents for selective cancer cell destruction. PNAS 102, 11600-11605 (2005).

69. Christopher J.Gannon, P.C., Boris I., Yakobson, Laurent Cognet John S., Kanzius Carter Kittrell ,R. Bruce Weisman, Matteo Pasquali, Howard K. Schmidt, Richard E. Smalley,Steven A. Curley Carbon nanotube-enhanced thermal destruction of cancer cells in a noninvasive radiofrequency field. Cancer 110, 2654-2665 (2007).

70. Wong Shi Kam, N. \& Dai, H. Single walled carbon nanotubes for transport and delivery of biological cargos. physica status solidi (b) 243, 3561-3566 (2006).

71. Cherukuri, P., Bachilo, S.M., Litovsky, S.H. \& Weisman, R.B. Near-Infrared Fluorescence Microscopy of Single-Walled Carbon Nanotubes in Phagocytic Cells. Journal of the American Chemical Society 126, 15638-15639 (2004).

72. Magrez, A.K., S. Salicio, V. Pasquier, N. Seo, J. W. Celio, M. Catsicas, S. Schwaller, B. Forro, L. Cellular toxicity of carbon-based nanomaterials. Nano Letters 6, 1121-1125 (2006). 
73. Bottini, M.B., S. Nika, K. Bottini, N. Bellucci, S. Magrini, A. Bergamaschi, A. Mustelin, T. Multi-walled carbon nanotubes induce $\mathrm{T}$ lymphocyte apoptosis. Toxicology Letters 160, 121-126 (2006).

74. Monteiro-Riviere, N.A., Nemanich, R.J., Inman, A.O., Wang, Y.Y. \& Riviere, J.E. Multi-walled carbon nanotube interactions with human epidermal keratinocytes. Toxicology Letters 155, 377-384 (2005).

75. Pulskamp, K.D., S.Krug, H. F. Carbon nanotubes show no sign of acute toxicity but induce intracellular reactive oxygen species in dependence on contaminants. Toxicology Letters 168, 58-74 (2007).

76. Davide Pantarotto, J.-P.B., Maurizio Prato , Alberto Bianco Translocation of bioactive peptides across cell membranes by carbon nanotubes. Chem. Commun, 16-17 (2004).

77. Dumortier H., L.S., Pastorin G, Marega R,Wu .W,Bonifazi D,Briand J. P,Prato M,Muller S.Bianco A. Functionalized Carbon Nanotubes Are Non-Cytotoxic and Preserve the Functionality of Primary Immune Cells. Nano Lett. 6, 1522-1528 (2006).

78. Daxiang Cui, F.T., Cengiz S. Ozkan, Mao Wang and Huajian Gao Effect of single wall carbon nanotubes on human HEK293 cells Toxicology Letters 155, 73-85 (2004).

79. Hadi N Yehia, R.K.D., Carole Mikoryak, Erin Kate Walker, Pooja Bajaj, Inga H Musselman, Meredith C Daigrepont, Gregg R Dieckmann,Paul Pantano Singlewalled carbon nanotube interactions with $\mathrm{HeLa}$ cells. Journal of Nanobiotechnology 5 (2007).

80. Chiu-Wing Lam, J.J., Richard McCluskey, Robert L. Hunter Pulmonary Toxicity of Single-Wall Carbon Nanotubes in Mice 7 and 90 Days After Intratracheal Instillation. TOXICOLOGICAL SCIENCES 77, 126-134 (2004).

81. Muller, J.H., F. Moreau, N. Misson, P. Heilier, J. F. Delos, M. Arras, M. Fonseca, A. Nagy, J. B. Lison, D. Respiratory toxicity of multi-wall carbon nanotubes. Toxicol Appl Pharmacol 207, 221-231 (2005).

82. Craig A. Poland, R.D., Ian Kinloch, Andrew Maynard,William A.H.Wallace, Anthony Seaton, Vicki Stone, Simon Brown,William Macnee, Ken Donaldson 
Carbon nanotubes introduced into the abdominal cavity ofmice show asbestoslike pathogenicity in a pilot study. Nat Nanotechnol 3 (2008).

83. Meike L. Schipper, N.N.-R., Corrine R. Davis,Nadine Wong Shi Kam, Pauline Chu, Zhaung Liu, Xiaoming Sun, Hongjie Dai, Sanjiv S.Gambhir A pilot toxicology study of single-walled carbon nanotubes in a small sample of mice. Nat Nanotechnol 3, 216-221 (2008).

84. Bai, Y.H. et al. Repeated administrations of carbon nanotubes in male mice cause reversible testis damage without affecting fertility. Nat Nanotechnol 5, 683-689 (2010).

85. Pulskamp, K., Diabaté, S. \& Krug, H.F. Carbon nanotubes show no sign of acute toxicity but induce intracellular reactive oxygen species in dependence on contaminants. Toxicology Letters 168, 58-74 (2007).

86. Hussain, S.M., Hess, K.L., Gearhart, J.M., Geiss, K.T. \& Schlager, J.J. In vitro toxicity of nanoparticles in BRL 3A rat liver cells. Toxicology in Vitro 19, 975983 (2005).

87. Davoren, $M$. et al. In vitro toxicity evaluation of single walled carbon nanotubes on human A549 lung cells. Toxicology in Vitro 21, 438-448 (2007).

88. Schipper, M.L. et al. A pilot toxicology study of single-walled carbon nanotubes in a small sample of mice. Nat Nano 3, 216-221 (2008).

89. Muller, J. et al. Respiratory toxicity of multi-wall carbon nanotubes. Toxicology and Applied Pharmacology 207, 221-231 (2005).

90. Gershon, R.K., Kruger, J., Naysmith, J.D. \& Waksman, B.H. Cellular basis for immunologic memory. Nature 232, 639-64l (1971).

91. Charles A Janeway, J., Paul Travers, Mark Walport, and Mark J Shlomchik. Immunobiology : the immune system in health and disease, Edn. 6th. (Garland Science, 2005).

92. Bruce Alberts, A.J., Julian Lewis, Martin Raff, Keith Roberts, and Peter Walter Molecular Biology of the Cell, Edn. 4th edition. (Garland Science, 2002). 
93. Lichtman, A.K.A.a.A.H. Basic Immunology: Functions and Disorders of the Immune System, Edn. 3rd. (Saunders, 2010).

94. Weissman, D. et al. Three populations of cells with dendritic morphology exist in peripheral blood, only one of which is infectable with human immunodeficiency virus type 1. Proceedings of the National Academy of Sciences 92, 826-830 (1995).

95. Zhou, L.J. \& Tedder, T.F. Human blood dendritic cells selectively express CD83, a member of the immunoglobulin superfamily. The Journal of Immunology 154, 3821-3835 (1995).

96. Zhou, L.J. \& Tedder, T.F. CD14+ blood monocytes can differentiate into functionally mature CD83+ dendritic cells. Proceedings of the National Academy of Sciences of the United States of America 93, 2588-2592 (1996).

97. Gorczyca, W. Acute promyelocytic leukemia: four distinct patterns by flow cytometry immunophenotyping. Pol J Pathol 63, 8-17 (2012).

98. Øbro, N.F. et al. Identification of residual leukemic cells by flow cytometry in childhood B-cell precursor acute lymphoblastic leukemia: verification of leukemic state by flow-sorting and molecular/cytogenetic methods. Haematologica 97, 137-141 (2012).

99. Barbesti, S. et al. A simplified flow cytometry method of CD4 and CD8 cell counting based on thermoresistant reagents: Implications for large scale monitoring of HIV-infected patients in resource-limited settings. Cytometry Part B: Clinical Cytometry 68B, 43-51 (2005).

100. Fromm, J.R.W., Brent L. Strategies for immunophenotyping and purifying classical Hodgkin lymphoma cells from lymph nodes by flow cytometry and flow cytometric cell sorting. Methods 57, 368-375 (2012).

101. Kuijpers, T.W. et al. Membrane surface antigen expression on neutrophils: a reappraisal of the use of surface markers for neutrophil activation. Blood $\mathbf{7 8}$, 1105-1111 (1991).

102. Sethu, P. et al. Microfluidic Isolation of Leukocytes from Whole Blood for Phenotype and Gene Expression Analysis. Analytical Chemistry 78, 5453-5461 (2006). 
103. Russom, A. et al. Microfluidic Leukocyte Isolation for Gene Expression Analysis in Critically Ill Hospitalized Patients. Clin Chem 54, $891-900$ (2008).

104. White, W., Raj, A., Nguyen, M.-D., Bertolone, S. \& Sethu, P. Clinical application of microfluidic leukocyte enrichment protocol in mild phenotype sickle cell disease (SCD). Biomedical Microdevices 11, 477-483 (2009).

105. Medepalli, K.A., B. Raj, A. Sethu, P. Evaluation of the direct and indirect response of blood leukocytes to carbon nanotubes (CNTs). Nanomedicine : nanotechnology, biology, and medicine 7, 983-991 (2011).

106. Lacerda, L., Bianco, A., Prato, M. \& Kostarelos, K. Carbon nanotubes as nanomedicines: From toxicology to pharmacology. Advanced Drug Delivery Reviews 58, 1460-1470 (2006).

107. Magrez, A. et al. Cellular Toxicity of Carbon-Based Nanomaterials. Nano Letters 6, 1121-1125 (2006).

108. Bottini, M. et al. Multi-walled carbon nanotubes induce T lymphocyte apoptosis. Toxicology Letters 160, 121-126 (2006).

109. Brus, L.E. Electron--electron and electron-hole interactions in small semiconductor crystallites: The size dependence of the lowest excited electronic state. The Journal of Chemical Physics 80, 4403-4409 (1984).

110. Alivisatos, A.P. Perspectives on the Physical Chemistry of Semiconductor Nanocrystals. The Journal of Physical Chemistry 100, 13226-13239 (1996).

111. Murray, C.B.K., C. R. Bawendi, M. G. Synthesis and characterization of monodisperse nanocrystals and close-packed nanocrystal assemblies. Annual Review of Materials Science 30, 545-610 (2000).

112. Bawendi, M.G.S., M L Brus, L E The Quantum Mechanics of Larger Semiconductor Clusters ("Quantum Dots"). Annual Review of Physical Chemistry 41, 477-496 (1990).

113. Cohen, M.L., Chou, M. Y.Knight, W. D.De Heer, Walt A. Physics of metal clusters. The Journal of Physical Chemistry 91, 3141-3149 (1987). 
114. Lutskii, V.N. Quantum size effect--present state and perspectives of experimental investigations. physica status solidi (a) 1, 199-220 (1970).

115. Lutskii, V.N.K., L. A. Features of Optical Characteristics of Bismuth Films Under Conditions of Quantum Size Effect. Journal of Experimental and Theoretical Physics Letters 8 (1968).

116. Filatov, O.N.K., I. A. Quantum Size Effects in Thin InSb Films. ZhETF Pis ma Redaktsiiu (Sov. Phys. Semicond.) 10 (1968).

117. Bimberg, D.G., Marius Ledentsov, Nikolai N. Quantum dot heterostructures. (John Wiley, Chichester, Eng. ; New York; 1999).

118. Vasko, F.T.K., Alex V. Electronic states and optical transitions in semiconductor heterostructures. (Springer, New York; 1999).

119. Zh. I. Alferov, Y.V.Z., and Yu. V. Shmartsev The splitting of the conduction band in 'superlattice' on the base of $\mathrm{GaP}_{x} \mathrm{As}_{1-x}$. Fiz. Tekh. Poluprovodn. (Sov. Phys. Semicond.) 5 (1971).

120. Kapon, E.H., D. M.Bhat, R. Stimulated emission in semiconductor quantum wire heterostructures. Physical Review Letters 63, 430-433 (1989).

121. Rocksby, H.P. The Colour of Selenium Ruby Glasses. J. Soc. Glass Tech. 16 (1932).

122. Efros, A.L., and A. L. Efros Interband absorption of light in a semiconductor sphere. Sov. Phys. Semicond. 16, 775-778 (1982).

123. Ekimov, A.I., and A. A. Onushchenko Quantum size effect in the optical-spectra of semiconductor micro-crystals. Sov. Phys. Semicond. 16, 16:775-778 (1982).

124. Kupka, R.K.C., Y.Planel, R.Launois, H. Fabrication of quantum wires and dots by $\mathrm{X}$-ray lithography and $\mathrm{Ga}+$ implantation enhanced intermixing. Microelectronic Engineering 27, 311-316 (1995).

125. Lorke, A.K., J. P. Ploog, K. Coupling of quantum dots on GaAs. Physical Review Letters 64, 2559-2562 (1990). 
126. Meurer, B.H., D.Ploog, K. Single-electron charging of quantum-dot atoms. Physical Review Letters 68, 1371-1374 (1992).

127. Freek E. Prins, G.L., Manfred Burkard, Sergej Yu. Nikitin, Heinz Schweizer and Gilbert W. Smith Quantum Dots and Quantum Wires with High Optical Quality by Implantation-Induced Intermixing. Jpn. J. Appl. Phys 32, 6228-6232 (1993).

128. Dybiec, M. in Electrical Engineering (University of South Florida, 2006).

129. S. Ishida, Y.A., K. Wada Seeded self-assembled GaAs quantum dots grown in two-dimensional $\mathrm{V}$ grooves by selective metal--organic chemical-vapor deposition. Applied Physics Letters 72, 800-802 (1998).

130. Y. Nagamune, M.N., S. Tsukamoto,Y. Arakawa GaAs quantum dots with lateral dimension of $25 \mathrm{~nm}$ fabricated by selective metalorganic chemical vapor deposition growth. Applied Physics Letters 64, 2495-2497 (1994).

131. Madhukar, A.R., K. C.Chen, P. In situ approach to realization of threedimensionally confined structures via substrate encoded size reducing epitaxy on nonplanar patterned substrates. Applied Physics Letters 62, 1547-1549 (1993).

132. Bhat, R.K., E. Hwang, D. M.Koza, M. A.Yun, C. P. Patterned quantum well heterostructures grown by OMCVD on non-planar substrates: Applications to extremely narrow SQW lasers. Journal of Crystal Growth 93, 850-856 (1988).

133. K. C. Rajkumar A. Madhukar P. Chen A. Konkar L. Chen K. Rammohan, D.H.R. Realization of three-dimensionally confined structures via one-step in $\square$ situ molecular beam epitaxy on appropriately patterned $\mathrm{GaAs}(111) \mathrm{B}$ and $\mathrm{GaAs}(001)$. J. Vac. Sci. Technol. B 12 (1994).

134. Stranski I. N ., K.L. Sitzungsberichte d. Akad. D. Wissenschaften in Wien. Abt. IIb, Band 146 (1937).

135. Hines, M.A. \& Guyot-Sionnest, P. Bright UV-Blue Luminescent Colloidal ZnSe Nanocrystals. The Journal of Physical Chemistry B 102, 3655-3657 (1998).

136. Dabbousi, B.O., Rodriguez-Viejo, J.Mikulec, F. V.Heine, J. R.Mattoussi, H.Ober, R.Jensen, K. F.Bawendi, M. G. (CdSe)ZnS Core-Shell Quantum Dots: $\square$ 
Synthesis and Characterization of a Size Series of Highly Luminescent Nanocrystallites. The Journal of Physical Chemistry B 101, 9463-9475 (1997).

137. Peng, X.S., Michael C.Kadavanich, Andreas V.,Alivisatos, A. P. Epitaxial Growth of Highly Luminescent $\mathrm{CdSe} / \mathrm{CdS}$ Core/Shell Nanocrystals with Photostability and Electronic Accessibility. Journal of the American Chemical Society 119, 7019-7029 (1997).

138. Murray, C.B.N., D. J. Bawendi, M. G. Synthesis and characterization of nearly monodisperse $\mathrm{CdE} \quad(\mathrm{E}=$ sulfur, selenium, tellurium) semiconductor nanocrystallites. Journal of the American Chemical Society 115, 8706-8715 (1993).

139. Manna, L., Milliron, D.J., Meisel, A., Scher, E.C. \& Alivisatos, A.P. Controlled growth of tetrapod-branched inorganic nanocrystals. Nature materials 2, 382-385 (2003).

140. Milliron, D.J. et al. Colloidal nanocrystal heterostructures with linear and branched topology. Nature 430, 190-195 (2004).

141. Dick, K.A. et al. Synthesis of branched 'nanotrees' by controlled seeding of multiple branching events. Nature materials 3, 380-384 (2004).

142. Yu, W.W.W., Y. A.Peng, X. G. Formation and stability of size-, shape-, and structure-controlled CdTe nanocrystals: Ligand effects on monomers and nanocrystals. Chemistry of Materials 15, 4300-4308 (2003).

143. Qu, L.H. \& Peng, X.G. Control of photoluminescence properties of CdSe nanocrystals in growth. Journal of the American Chemical Society 124, 20492055 (2002).

144. Bailey, R.E. \& Nie, S. Alloyed Semiconductor Quantum Dots: $\square$ Tuning the Optical Properties without Changing the Particle Size. Journal of the American Chemical Society 125, 7100-7106 (2003).

145. LaMer, V.K. \& Dinegar, R.H. Theory, Production and Mechanism of Formation of Monodispersed Hydrosols. Journal of the American Chemical Society 72, 4847-4854 (1950). 
146. De Smet, Y., Deriemaeker, L. \& Finsy, R. A Simple Computer Simulation of Ostwald Ripening. Langmuir 13, 6884-6888 (1997).

147. Grätz, H. Ostwald ripening: New relations between particle growth and particle size distribution. Scripta Materialia 37, 9-16 (1997).

148. Yu, W.W., Qu, L., Guo, W. \& Peng, X. Experimental Determination of the Extinction Coefficient of CdTe, CdSe, and CdS Nanocrystals. Chemistry of Materials 15, 2854-2860 (2003).

149. Talapin, D.V.R., Andrey L. Mekis, Ivo Haubold, Stephan Kornowski, Andreas Haase, Markus Weller, Horst Synthesis and surface modification of aminostabilized CdSe, CdTe and InP nanocrystals. Colloids and Surfaces A: Physicochemical and Engineering Aspects 202, 145-154 (2002).

150. Zhong, X., Han, M., Dong, Z., White, T.J. \& Knoll, W. Composition-Tunable ZnxCd1-xSe Nanocrystals with High Luminescence and Stability. Journal of the American Chemical Society 125, 8589-8594 (2003).

151. Peng, X. Mechanisms for the Shape-Control and Shape-Evolution of Colloidal Semiconductor Nanocrystals. Advanced Materials 15, 459-463 (2003).

152. Qu, L., Peng, Z.A. \& Peng, X. Alternative Routes toward High Quality CdSe Nanocrystals. Nano Letters 1, 333-337 (2001).

153. Talapin, D.V., Rogach, A.L., Kornowski, A., Haase, M. \& Weller, H. Highly Luminescent Monodisperse CdSe and CdSe/ZnS Nanocrystals Synthesized in a Hexadecylamine-Trioctylphosphine Oxide-Trioctylphospine Mixture. Nano Letters 1, 207-211 (2001).

154. Chen, H.-S. et al. Colloidal $\mathrm{ZnSe}, \mathrm{ZnSe} / \mathrm{ZnS}$, and $\mathrm{ZnSe} / \mathrm{ZnSeS}$ Quantum Dots Synthesized from ZnO. The Journal of Physical Chemistry B 108, 17119-17123 (2004).

155. Talapin, D.V. et al. CdSe/CdS/ZnS and CdSe/ZnSe/ZnS Core-Shell-Shell Nanocrystals. The Journal of Physical Chemistry B 108, 18826-18831 (2004).

156. Cheng, C.-T.C., Chun-Yen Lai, Chih-Wei Liu, Wei-Hsin Pu, Shih-Chieh Chou, Pi-Tai Chou, Yi-Hsuan Chiu, Hsin-Tien Syntheses and photophysical properties 
of type-II CdSe/ZnTe/ZnS (core/shell/shell) quantum dots. Journal of Materials Chemistry 15, 3409-3414 (2005).

157. Kim, S., Fisher, B., Eisler, H.-J. \& Bawendi, M. Type-II Quantum Dots: $\mathrm{CdTe} / \mathrm{CdSe}(\mathrm{Core} / \mathrm{Shell})$ and $\mathrm{CdSe} / \mathrm{ZnTe}(\mathrm{Core} / \mathrm{Shell})$ Heterostructures. Journal of the American Chemical Society 125, 11466-11467 (2003).

158. Reiss, P., Protiere, M. \& Li, L. Core/Shell Semiconductor Nanocrystals. Small 5, 154-168 (2009).

159. Chan, W.C.W.N., Shuming Quantum Dot Bioconjugates for Ultrasensitive Nonisotopic Detection. Science 281, 2016-2018 (1998).

160. Bruchez, M., Moronne, M., Gin, P., Weiss, S. \& Alivisatos, A.P. Semiconductor Nanocrystals as Fluorescent Biological Labels. Science 281, 2013-2016 (1998).

161. Medintz, I.L.U., H. Tetsuo Goldman, Ellen R. Mattoussi, Hedi Quantum dot bioconjugates for imaging, labelling and sensing. Nat Mater 4, 435-446 (2005).

162. Mattoussi, H. et al. Self-Assembly of $\mathrm{CdSe}-\mathrm{ZnS}$ Quantum Dot Bioconjugates Using an Engineered Recombinant Protein. Journal of the American Chemical Society 122, 12142-12150 (2000).

163. Mitchell, G.P.M., Chad A. Letsinger, Robert L. Programmed Assembly of DNA Functionalized Quantum Dots. Journal of the American Chemical Society 121, 8122-8123 (1999).

164. Goldman, E.R. et al. Avidin: A Natural Bridge for Quantum Dot-Antibody Conjugates. Journal of the American Chemical Society 124, 6378-6382 (2002).

165. Uyeda, H.T., Medintz, I.L., Jaiswal, J.K., Simon, S.M. \& Mattoussi, H. Synthesis of Compact Multidentate Ligands to Prepare Stable Hydrophilic Quantum Dot Fluorophores. Journal of the American Chemical Society 127, 3870-3878 (2005).

166. Dubertret, B. et al. In Vivo Imaging of Quantum Dots Encapsulated in Phospholipid Micelles. Science 298, 1759-1762 (2002). 
167. Wu, X.L., H. Liu, J. Haley, K. N.Treadway, J. A.Larson, J. P.Ge, N.Peale, F.Bruchez, M. P. Immunofluorescent labeling of cancer marker Her2 and other cellular targets with semiconductor quantum dots. Nat Biotechnol 21, 41-46 (2003).

168. Pellegrino, T. et al. Hydrophobic Nanocrystals Coated with an Amphiphilic Polymer Shell: $\square$ A General Route to Water Soluble Nanocrystals. Nano Letters 4, 703-707 (2004).

169. Gao, X., Cui, Y., Levenson, R.M., Chung, L.W.K. \& Nie, S. In vivo cancer targeting and imaging with semiconductor quantum dots. Nat Biotech 22, 969-976 (2004).

170. Gerion, D. et al. Synthesis and Properties of Biocompatible Water-Soluble SilicaCoated CdSe/ZnS Semiconductor Quantum Dots $\dagger$. The Journal of Physical Chemistry B 105, 8861-8871 (2001).

171. Smith, A.M. \& Nie, S. Chemical analysis and cellular imaging with quantum dots. Analyst 129, 672-677 (2004).

172. Leatherdale, C.A.W., W. K. Mikulec, F. V. Bawendi, M. G. On the Absorption Cross Section of CdSe Nanocrystal Quantum Dots. The Journal of Physical Chemistry $B$ 106, 7619-7622 (2002).

173. Nie, S. Imaging dynamic cellular events with quantum dots The bright future. Biochem(London) 32, 12 (2010).

174. Gao, X.Y., Lily Petros, John A. Marshall, Fray F. Simons, Jonathan W. Nie, Shuming In vivo molecular and cellular imaging with quantum dots. Current Opinion in Biotechnology 16, 63-72 (2005).

175. Hermanson, G.T. Bioconjugate techniques, Edn. 2. (Academic Press, 2008).

176. Goldman, E.R.A., George P. Tran, Phan T. Mattoussi, Hedi Charles, Paul T.Mauro, J. Matthew Conjugation of Luminescent Quantum Dots with Antibodies Using an Engineered Adaptor Protein To Provide New Reagents for Fluoroimmunoassays. Anal Chem 74, 841-847 (2002). 
177. Willner, I.P., Fernando Wasserman, Julian Photoelectrochemistry with Controlled DNA-Cross-Linked CdS Nanoparticle Arrays. Angewandte Chemie International Edition 40, 1861-1864 (2001).

178. Mitchell, G.P., Mirkin, C.A. \& Letsinger, R.L. Programmed assembly of DNA functionalized quantum dots. Journal of the American Chemical Society 121, 8122-8123 (1999).

179. Pathak, S.C., S. K. Arnheim, N. Thompson, M. E. Hydroxylated quantum dots as luminescent probes for in situ hybridization. Journal of the American Chemical Society 123, 4103-4104 (2001).

180. Parak, W.J.B., R. Le Gros, M. Gerion, D. Zanchet, D. Micheel, C. M. Williams, S. C. Alivisatos, A. P. Larabell, C. Cell Motility and Metastatic Potential Studies Based on Quantum Dot Imaging of Phagokinetic Tracks. Advanced Materials 14, 882-885 (2002).

181. Dahan, M.L., S. Luccardini, C. Rostaing, P. Riveau, B. Triller, A. Diffusion dynamics of glycine receptors revealed by single-quantum dot tracking. Science 302, 442-445 (2003).

182. Levene, M.J., Dombeck, D.A., Kasischke, K.A., Molloy, R.P. \& Webb, W.W. In Vivo Multiphoton Microscopy of Deep Brain Tissue. Journal of Neurophysiology 91, 1908-1912 (2004).

183. Rosenthal, S.J. et al. Targeting cell surface receptors with ligand-conjugated nanocrystals. Journal of the American Chemical Society 124, 4586-4594 (2002).

184. Kim, S.L., Y. T. Soltesz, E. G. De Grand, A. M. Lee, J. Nakayama, A. Parker, J. A. Mihaljevic, T. Laurence, R. G. Dor, D. M. Cohn, L. H. Bawendi, M. G. Frangioni, J. V. Near-infrared fluorescent type II quantum dots for sentinel lymph node mapping. Nat Biotechnol 22, $93-97$ (2004).

185. Voura, E.B., Jaiswal, J.K., Mattoussi, H. \& Simon, S.M. Tracking metastatic tumor cell extravasation with quantum dot nanocrystals and fluorescence emission-scanning microscopy. Nat Med 10, 993-998 (2004).

186. Gokarna, A.J., Li-Hua Hwang, Jun Seok Cho, Yong-Hoon Lim, Yong Taik Chung, Bong Hyun Youn, Seong Hun Choi, Dong Sik Lim, Jung Hyurk Quantum 
dot-based protein micro- and nanoarrays for detection of prostate cancer biomarkers. Proteomics 8, 1809-1818 (2008).

187. Chornokur, G. et al. Scanning photoluminescent spectroscopy of bioconjugated quantum dots. Superlattices and Microstructures 45, 240-248 (2009).

188. Wagner, M., Li, F., Li, J., Li, X.-F. \& Le, X. Use of quantum dots in the development of assays for cancer biomarkers. Analytical and Bioanalytical Chemistry 397, 3213-3224 (2010).

189. Yang, R.S.C., L. W.Wu, J. P.Tsai, M. H.Wang, H. J.Kuo, Y. C.Yeh, T. K.Yang, C. S.Lin, P Persistent tissue kinetics and redistribution of nanoparticles, quantum dot 705, in mice: ICP-MS quantitative assessment. Environ Health Perspect 115, 1339-1343 (2007).

190. Cho, S.J. et al. Long-term exposure to CdTe quantum dots causes functional impairments in live cells. Langmuir 23, 1974-1980 (2007).

191. Chang, E.T., N. Yu, W. W. Colvin, V. L. Drezek, R. Evaluation of quantum dot cytotoxicity based on intracellular uptake. Small 2, 1412-1417 (2006).

192. Derfus, A.M., Chan, W.C.W. \& Bhatia, S.N. Probing the Cytotoxicity of Semiconductor Quantum Dots. Nano Letters 4, 11-18 (2003).

193. Pradhan, N. \& Peng, X. Efficient and color-tunable Mn-doped ZnSe nanocrystal emitters: control of optical performance via greener synthetic chemistry. Journal of the American Chemical Society 129, 3339-3347 (2007).

194. Pradhan, N., Battaglia, D.M., Liu, Y. \& Peng, X. Efficient, Stable, Small, and Water-Soluble Doped ZnSe Nanocrystal Emitters as Non-Cadmium Biomedical Labels. Nano Letters 7, 312-317 (2006).

195. Hayden, M.S. \& Ghosh, S. Signaling to NF-kappaB. Genes Dev 18, 2195-2224 (2004).

196. Chen, L.F. \& Greene, W.C. Shaping the nuclear action of NF-kappaB. Nat Rev Mol Cell Biol 5, 392-401 (2004). 
197. Kumar, A.T., Yasunari Boriek, Aladin M. Aggarwal, Bharat B. Nuclear factorkB: its role in health and disease. Journal of Molecular Medicine 82, 434-448 (2004).

198. Magne, N.T., R. A. Bottero, V. Didelot, C. Houtte, P. V. Gerard, J. P. Peyron, J. F. NF-kappaB modulation and ionizing radiation: mechanisms and future directions for cancer treatment. Cancer Lett 231, 158-168 (2006).

199. Li, N. \& Karin, M. Ionizing radiation and short wavelength UV activate NF-kB through two distinct mechanisms. Proceedings of the National Academy of Sciences 95, 13012-13017 (1998).

200. Hamid, T.G., Y.Ortines, R. V.Bhattacharya, C.Wang, G.Xuan, Y. T.Prabhu, S. D. Divergent tumor necrosis factor receptor-related remodeling responses in heart failure: role of nuclear factor-kappaB and inflammatory activation. Circulation 119, 1386-1397 (2009).

201. Fuseler, J.W., Merrill, D.M., Rogers, J.A., Grisham, M.B. \& Wolf, R.E. Analysis and Quantitation of NF-[kappa]B Nuclear Translocation in Tumor Necrosis Factor Alpha (TNF-[alpha]) Activated Vascular Endothelial Cells. Microscopy and Microanalysis 12, 269-276 (2006).

202. Albert S. Baldwin, J. Series Introduction-The transcription factor NF- $\kappa B$ and human disease. J Clin Invest 107 (2001).

203. Swaroop, A.D.L., Nelson NF- $\mathrm{kB}$ signaling and human disease. Current Opinion in Genetics \& Development 11, 300-306 (2001).

204. Fulvio D'Acquisto, M.J.M., and Sankar Ghosh Inhibition of Nuclear Factor Kappa B (NF-kB)-an emerging theme in anti inflammatory therapies. 2, 22-35 (2002).

205. Makarov, S.S. NF- $\mathrm{kB}$ as a therapeutic target in chronic inflammation- recent advances. Molecular medicine today 6, 441-448 (2000).

206. Yumi , Y.R., B. Gaynor Therapeutic potential of inhibition of the NF- $\mathrm{kB}$ pathway in the treatment of inflammation and cancer. J Clin Invest 107 (2001). 
207. Hamid, T.G., S. Z. Kingery, J. R. Xiang, X. Dawn, B. Prabhu, S. D. Cardiomyocyte NF-kappaB p65 promotes adverse remodelling, apoptosis, and endoplasmic reticulum stress in heart failure. Cardiovasc Res 89, 129-138 (2011).

208. Nishikawa, M. \& Huang, L. Nonviral vectors in the new millennium: delivery barriers in gene transfer. Hum Gene Ther 12, 861-870 (2001).

209. Niidome, T. \& Huang, L. Gene therapy progress and prospects: nonviral vectors. Gene therapy 9, 1647-1652 (2002).

210. Pannier, A.K. \& Shea, L.D. Controlled Release Systems for DNA Delivery. Mol Ther 10, 19-26 (2004).

211. Biju, V.I., Tamitake Ishikawa, Mitsuru Delivering quantum dots to cells: bioconjugated quantum dots for targeted and nonspecific extracellular and intracellular imaging. Chemical Society Reviews 39, 3031-3056 (2010).

212. Anas, A. et al. Clathrin-mediated endocytosis of quantum dot-peptide conjugates in living cells. ACS Nano 3, 2419-2429 (2009).

213. Swanson, J.A. \& Watts, C. Macropinocytosis. Trends in Cell Biology 5, 424-428 (1995).

214. Okada, C.Y. \& Rechsteiner, M. Introduction of macromolecules into cultured mammalian cells by osmotic lysis of pinocytic vesicles. Cell 29, 33-41 (1982).

215. Courty, S., Luccardini, C., Bellaiche, Y., Cappello, G. \& Dahan, M. Tracking Individual Kinesin Motors in Living Cells Using Single Quantum-Dot Imaging. Nano Letters 6, 1491-1495 (2006).

216. Derfus, A.M., Chan, W.C.W. \& Bhatia, S.N. Intracellular Delivery of Quantum Dots for Live Cell Labeling and Organelle Tracking. Advanced Materials 16, 961-966 (2004).

217. Ryman-Rasmussen, J.P., Riviere, J.E. \& Monteiro-Riviere, N.A. Variables influencing interactions of untargeted quantum dot nanoparticles with skin cells and identification of biochemical modulators. Nano Letters 7, 1344-1348 (2007). 
218. Mok, H., Park, J.W. \& Park, T.G. Enhanced intracellular delivery of quantum dot and adenovirus nanoparticles triggered by acidic $\mathrm{pH}$ via surface charge reversal. Bioconjugate Chemistry 19, 797-801 (2008).

219. Jaiswal, J.K.M., Hedi Mauro, J. Matthew Simon, Sanford M. Long-term multiple color imaging of live cells using quantum dot bioconjugates. Nat Biotech 21, 4751 (2003).

220. Duan, H. \& Nie, S. Cell-Penetrating Quantum Dots Based on Multivalent and Endosome-Disrupting Surface Coatings. Journal of the American Chemical Society 129, 3333-3338 (2007).

221. Kim, B.Y. et al. Biodegradable quantum dot nanocomposites enable live cell labeling and imaging of cytoplasmic targets. Nano Letters 8, 3887-3892 (2008).

222. Ruan, G., Agrawal, A., Marcus, A.I. \& Nie, S. Imaging and Tracking of Tat Peptide-Conjugated Quantum Dots in Living Cells: $\square$ New Insights into Nanoparticle Uptake, Intracellular Transport, and Vesicle Shedding. Journal of the American Chemical Society 129, 14759-14766 (2007).

223. Weaver, J.C. Electroporation: a general phenomenon for manipulating cells and tissues. J Cell Biochem 51, 426-435 (1993).

224. Chen, X., Kis, A., Zettl, A. \& Bertozzi, C.R. A cell nanoinjector based on carbon nanotubes. Proceedings of the National Academy of Sciences 104, 8218-8222 (2007).

225. Hapala, I. Breaking the Barrier: Methods for Reversible Permeabilization of Cellular Membranes. Critical Reviews in Biotechnology 17, 105-122 (1997).

226. van de Ven, A.L., Adler-Storthz, K. \& Richards-Kortum, R. Delivery of optical contrast agents using Triton-X100, part 1: reversible permeabilization of live cells for intracellular labeling. Journal of Biomedical Optics 14, 021012 (2009).

227. Goldenthal, K.L.H., K Chen, J W August, J T Willingham, M C Postfixation detergent treatment for immunofluorescence suppresses localization of some integral membrane proteins. Journal of Histochemistry \& Cytochemistry 33, 813820 (1985). 
228. Miyamoto, K. et al. Reversible membrane permeabilization of mammalian cells treated with digitonin and its use for inducing nuclear reprogramming by Xenopus egg extracts. Cloning Stem Cells 10, 535-542 (2008).

229. Adam, S.A., Marr, R.S. \& Gerace, L. Nuclear protein import in permeabilized mammalian cells requires soluble cytoplasmic factors. $J$ Cell Biol 111, 807-816 (1990).

230. Adam, S.A.S.-M., Rachel Gerace, Larry in Methods in enzymology, Vol. Volume 219. (ed. E.R. James) 97-110 (Academic Press, 1992).

231. Karl, J. et al. GFAP phosphorylation studied in digitonin-permeabilized astrocytes: standardization of conditions. Brain Res 853, 32-40 (2000).

232. Lowe, A.R. et al. Selectivity mechanism of the nuclear pore complex characterized by single cargo tracking. Nature 467, 600-603 (2010).

233. Tschesche, V.R. \& Wulff, G. [Chemistry and biology of saponins]. Fortschr Chem Org Naturst 30, 461-606 (1973).

234. Schlosser, E. \& Wulff, G. [Structural specificity of saponin hemolysis. I. Triterpene saponins and aglycones]. Z Naturforsch B 24, 1284-1290 (1969).

235. Bangham, A.D., Horne, R.W., Glauert, A.M., Dingle, J.T. \& Lucy, J.A. Action of saponin on biological cell membranes. Nature 196, 952-955 (1962).

236. Brooks, J.C. \& Carmichael, S.W. Scanning Electron-Microscopy of Chemically Skinned Bovine Adrenal-Medullary Chromaffin Cells. Mikroskopie 40, 347-356 (1983).

237. Wassler, M., Jonasson, I., Persson, R. \& Fries, E. Differential permeabilization of membranes by saponin treatment of isolated rat hepatocytes. Release of secretory proteins. Biochem J 247, 407-415 (1987).

238. Negrutskii, B.S., Stapulionis, R. \& Deutscher, M.P. Supramolecular organization of the mammalian translation system. Proceedings of the National Academy of Sciences 91, 964-968 (1994). 
239. Hudder, A., Nathanson, L. \& Deutscher, M.P. Organization of Mammalian Cytoplasm. Molecular and Cellular Biology 23, 9318-9326 (2003).

240. Johnson, J.A., Gray, M.O., Karliner, J.S., Chen, C.-H. \& Mochly-Rosen, D. An Improved Permeabilization Protocol for the Introduction of Peptides Into Cardiac Myocytes: Application to Protein Kinase C Research. Circulation Research 79, 1086-1099 (1996).

241. Mukherjee, S., Ghosh, R.N. \& Maxfield, F.R. Endocytosis. Physiological Reviews 77, 759-803 (1997).

242. Sethu, P. Microfluidic Isolation of Leukocytes from Whole Blood for Phenotype and Gene Expression Analysis. Anal Chem 78, 5453-5461.

243. Parekkadan, B., Sethu, P., van Poll, D., Yarmush, M.L. \& Toner, M. Osmotic selection of human mesenchymal stem/progenitor cells from umbilical cord blood. Tissue Eng 13, 2465-2473 (2007).

244. Parichehreh, V.E., Rosendo Kumar, Srikanth Bhavanam, Kranthi Raj, Vinay Raj, Ashok Sethu, Palaniappan Exploiting osmosis for blood cell sorting. Biomedical Microdevices 13, 453-462 (2011).

245. Lemoine, J.L., Farley, R. \& Huang, L. Mechanism of efficient transfection of the nasal airway epithelium by hypotonic shock. Gene Ther 12, 1275-1282 (2005).

246. Cholpon S. Djuzenova, J.K., Martin Kiesel, Lavinia Stingl, Ulrich Zimmermann, Michael Flentje, Vladimir L. Sukhorukov Intracellular delivery of 2-deoxy-Dglucose into tumor cells by long-term cultivation and through swelling-activated pathways: implications for radiation treatment. Molecular Medicine Reports 2, 633-640 (2009).

247. Kam, N.W.S., O'Connell, M., Wisdom, J.A. \& Dai, H. Carbon nanotubes as multifunctional biological transporters and near-infrared agents for selective cancer cell destruction. Proc Natl Acad Sci U S A 102, 11600-11605 (2005). 


\title{
CURRICULUM VITAE
}

\author{
MEDEPALLI, KRISHNA KIRAN \\ 1800, South 2nd Street, Apt \# A42 \\ Louisville, KY 40208 \\ Phone: (502)-751-2323
}

Email: k0mede01@louisville.edu

\section{RESEARCH INTERESTS}

Micro/nano-fabrication, Synthesis and characterization (electrical, optical and biological) of nanomaterials. Nanomaterials and Microfluidic systems for nano-biotechnology applications. Specifically, integration of nanomaterials such as colloidal crystals, carbon based nanomaterials and nanoparticles for applications in biomedical, nanomedicine and microfluidics technologies.

\section{EDUCATION}

2008-present: $\mathrm{Ph}$.D. Electrical and Computer Engineering

University of Louisville, Louisville, KY

Advisor: Prof. Bruce W.Alphenaar

Thesis: Nanomaterials for cellular delivery and imaging of biomolecules with subcellular resolution.

2005-2008 : M.S. Electrical and Computer Engineering

University of Louisville, Louisville, KY

Advisor: Prof Bruce W.Alphenaar

Thesis: Advanced Nanomaterials for Biomedical Applications

2001-2005 : B. Tech. Electrical and Electronics Engineering

Jawaharlal Nehru Technological University, Hyderabad, India

\section{TECHNICAL EXPERTISE}

- Cleanroom techniques including

- Lithography: Optical lithography, Soft lithography and E-beam lithography.

- Etching: Wet etching (HF, $\mathrm{HCl}$, Nano-strip), Reactive Ion etching. 
o Thin film Deposition: RF/DC sputter, Thermal/E-beam evaporation, Selfassembly, Spin coating.

- Chemical Vapor Deposition (CVD): Carbon nanotubes and Graphene.

- Material characterization: 7 years of experience with SEM, AFM, XRD, FTIR, UVVis-NIR and Raman spectroscopy, Energy dispersive spectroscopy, visible and NIR photoluminescence, fluorescence spectroscopy, Dynamic light scattering, Capacitive photocurrent spectroscopy (CPS) and NIR microscopy.

- Biomedical laboratory skills: Cell culture, Electrophoresis, ELISA, protein extraction and estimation, immunofluorescence, fluorescent labeling of proteins and antibodies, confocal, fluorescence microscopy and flow cytometry.

- Dispersions: Carbon nanotubes, nanoparticles (Gold, Silica), QDs.

- Hands on experience in design, fabrication and development of microfluidic systems for cellular and biomolecular analysis.

- Programming and Software packages: LabView, Matlab, Fortran, Pspice, L-edit, IGOR, Image J, Metamorph, Photoshop, Peakfit, WIN MDI, Cell Quest Pro.

\section{RESEARCH EXPERIENCE}

Graduate Research Assistant, Nanoscale Device Research Laboratory, Department of Electrical engineering, University of Louisville, (July 2008-present)

- Synthesis and characterization of carbon nanotubes and nanoparticles.

- Designed and assembled a complete photoluminescence system for the optical spectroscopy of carbon nanotubes and QDs.

- Optical and biological characterization of nanomaterials such as carbon nanotubes, nanoparticles. (Gold, Silica and QDs)

- Dispersion and separation of carbon nanotubes by density gradient centrifugation.

Graduate Research Assistant, Microscale Biotechnology Laboratory, Department of Bioengineering, University of Louisville, (Sep 2008-present)

- Isolation of peripheral blood mononuclear cells (PBMCs) from whole blood and characterization of their interactions with nanomaterials using flow cytometry and confocal microscopy.

- Development of real time imaging and detection of subcellular NFkB protein localization in live cells using quantum dots.

- Design, fabrication and development of lab on chip microfluidic systems for labeling, separation and detection of cells. 
- Surface functionalization of carbon nanotubes and nanoparticles (Silica, QDs) with DNA, antibodies, proteins and other biomaterials.

Graduate Research Assistant, Gheen's center for Aging, Department of Biochemistry, University of Louisville, (Jan 2006- Jul 2008)

- Functionalization of self-assembled layers of Silica colloidal crystals with biomaterials such as DNA and collagen.

- Development of a novel method for localized drug delivery of DNA using colloidal Silica crystals.

Department Student Assistant, Department of Electrical and Computer Engineering, University of Louisville, (Aug 2005- Dec 2005)

- Assisted the department of Electrical and Computer engineering grading the course Introduction to Electromagnetic Fields.

\section{TEACHING EXPERIENCE}

- Math tutor in Resources for Academic Achievement (REACH), University of Louisville (Sep 2005 to Dec 2005).

- Taught first year engineering students concepts of Electrical and Electronic engineering, (Jan 2004 to Aug 2005), Hyderabad, India.

\section{PEER REVIEWED PUBLICATIONS}

1. Medepalli. K, Alphenaar. B, Keynton RS, Sethu. P, "Nanotechnology Based Approaches to Study Intracellular Signaling", Fifth Annual, Nanotechnology and Nanomedicine Symposium, Sullivan University College of Health Sciences, College of Pharmacy, (submitted) September 2012.

2. Medepalli. K, Alphenaar. B, Keynton RS, Sethu. P, "A New Technique for Reversible Permeabilization of Live Cells for Intracellular Delivery of Quantum Dots" (Under review, Nanomedicine), August 2012

3. Medepalli. K, Alphenaar. B, Keynton. RS, Sethu. P, "Reversible permeabilization of live cells for intracellular delivery of semiconductor quantum dots", 1st Annual International Space Station Research and Development Conference, 2012, Denver CO (manuscript submitted), July 2012

4. Parichehreh. V, Medepalli. K, Babbarwal. K, Sethu. P, "Microfluidic inertia enhanced phase partitioning for enriching circulating tumor cells (CTCs) in blood" (Under review in Lab on a Chip), 2012.

5. Medepalli K, Alphenaar B, Raj A, Sethu P, "Demonstration of biocompatibility of single walled carbon nanotubes with blood leukocytes", Materials Research Society (MRS) Online Proceedings Library, 1416, Feb 2012. 
6. Medepalli K, Alphenaar B, Raj A, Sethu P, "Evaluation of the direct and indirect response of blood leukocytes to carbon nanotubes (CNTs)", Nanomedicine, May 2011, 7(6), 983-991.

7. Sarojini.H, Medepalli.K, Derek.A Terry, Bruce W. Alphenaar, Eugenia Wang, "Localized delivery of DNA to the cells by viral collagen-loaded silica colloidal crystals", Journal of Biotechniques, Aug 2007, 43(2), 213-221.

\section{CONFERENCE PRESENTATIONS AND ABSTRACTS}

1. Medepalli. K, Alphenaar. B, Keynton. R, Sethu. P, "Permeabilization techniques for the intracellular delivery of quantum dots in live cells", Biomedical Engineering Society (BMES) Annual Meeting, Atlanta, GA, 2012.

2. Medepalli. K, Alphenaar. B, Keynton. R, Sethu. P, "Techniques for Detection of NFKB In Live Cells", $10^{\text {th }}$ Annual OVALS Conference, 2012, Louisville, KY

3. Medepalli.K, Bruce Alphenaar, Ashok Raj and Palaniappan Sethu, "Demonstration of biocompatibility of single walled carbon nanotubes with blood leukocytes", Biomedical Research Showcase, University of Louisville, 2012, Louisville, KY

4. Medepalli.K, Palaniappan Sethu, "Intracellular delivery of quantum dot antibody conjugates using reversible permeabilization techniques", NASA Biomarker Discovery Group Quarterly Progress report, University of Louisville, 2012, Louisville, KY.

5. Medepalli. K, Alphenaar. B, Keynton. RS, Sethu. P, "Reversible permeabilization of live cells for intracellular delivery of semiconductor quantum dots", 1st Annual International Space Station Research and Development Conference, 2012, Denver CO (abstract accepted)

6. Medepalli.K, Brigitte Fasciotto, Phani Patibandla, Palaniappan Sethu, "Real-time imaging and detection of sub-cellular NF-kB localization in cells", NASA Biomarker Discovery Group Quarterly Progress report, University of Louisville, 2011, Louisville, KY.

7. Medepalli.K, Bruce Alphenaar, Ashok Raj and Palaniappan Sethu, "Demonstration of biocompatibility of single walled carbon nanotubes with blood leukocytes", Material Research Society (MRS) Fall meeting, 2011, Boston, MA.

8. Medepalli K, Alphenaar B, Raj A, Sethu P, "Evaluation of the direct and indirect response of blood leukocytes to carbon nanotubes (CNTs)", Research Louisville! 2011, Louisville, KY.

9. Buddika K. Abeyweera, H. Shah, T. Bansal, Medepalli K. \& B. W. Alphenaar "Fabrication techniques for high efficient organic polymer solar cell", Engineers Day, 2010, Louisville KY. 
10. Medepalli.K, A.D.Mohite, Sarojini.H, Bruce W. Alphenaar, Eugenia Wang "Carbon nanotubes as biological transporters for intracellular drug delivery", Engineers Day, 2008 \& KY NANOMAT 2008, Louisville, KY.

11. Medepalli.K, A.D.Mohite, Sarojini.H, Bruce W. Alphenaar, Eugenia Wang "Advance Nanomaterials for efficient intracellular drug delivery", Engineers Day, 2007, Louisville KY.

12. Sarojini.H, Medepalli.K, Derek.A Terry, Bruce W. Alphenaar, Eugenia Wang "Delivery of recombinant DNA to target-subpopulation of cells in monolayer culture using a viral collagen-loaded colloidal crystal platform", Research Louisville! 2006, Louisville, KY.

13. Sreenath Arva, Medepalli.K, Bruce W. Alphenaar, G. Sumanasekera, "Novel Devices based on the colloidal crystal templating method", at $12^{\text {th }}$ Annual Kentucky EPSCoR Conference 2006, Louisville, KY.

14. Kona.S, Medepalli.K, "Cognizance of Technology", Infoquest 2004, National Level Technical Symposium, Hyderabad, India.

15. Medepalli.K, Kona.S, "Applications of Control Systems in the field of Avionics", Vihaan 2004, National Level Technical Symposium, Hyderabad, India.

\section{INVENTION DISCLOSURES}

- Krishna Kiran Medepalli, Palaniappan Sethu, Bruce Alphenaar, "Techniques for detection of NFkB in blood leukocytes and live cells", Aug 2011, Disclosure filed.

\section{ACHIEVEMENTS AND AWARDS}

- Dissertation Completion Award, University of Louisville, Aug 2012-Dec 2012.

- University Fellowship from the University of Louisville, Aug 2008-2010.

- Highest place in PhD qualifying exam, University of Louisville, 2009.

- Graduate Research Assistantship, University of Louisville, Jan 2006- Jul 2008.

- First prize Infoquest 2004, National Level Technical Symposium, Hyderabad, India.

- First Prize Vihaan 2004, National Level Technical Symposium, Hyderabad, India.

\section{AFFILIATIONS/MEMBERSHIPS}

- Biomedical Engineering Society (BMES)

- Material Research Society (MRS)

- Indian Student Association (ISA), University of Louisville. 
- Louisville Badminton club. 